\title{
Pleiotropic effects of non-structural matrix proteins in the stressed heart : ECM remodeling in cardiotoxicity, aging and cardiac allograft rejection
}

Citation for published version (APA):

van Almen, G. C. (2012). Pleiotropic effects of non-structural matrix proteins in the stressed heart: ECM remodeling in cardiotoxicity, aging and cardiac allograft rejection. [Doctoral Thesis, Maastricht University]. Datawyse / Universitaire Pers Maastricht. https://doi.org/10.26481/dis.20120516ga

Document status and date:

Published: 01/01/2012

DOI:

10.26481/dis.20120516ga

Document Version:

Publisher's PDF, also known as Version of record

Please check the document version of this publication:

- A submitted manuscript is the version of the article upon submission and before peer-review. There can be important differences between the submitted version and the official published version of record.

People interested in the research are advised to contact the author for the final version of the publication, or visit the DOI to the publisher's website.

- The final author version and the galley proof are versions of the publication after peer review.

- The final published version features the final layout of the paper including the volume, issue and page numbers.

Link to publication

\footnotetext{
General rights rights.

- You may freely distribute the URL identifying the publication in the public portal. please follow below link for the End User Agreement:

www.umlib.nl/taverne-license

Take down policy

If you believe that this document breaches copyright please contact us at:

repository@maastrichtuniversity.nl

providing details and we will investigate your claim.
}

Copyright and moral rights for the publications made accessible in the public portal are retained by the authors and/or other copyright owners and it is a condition of accessing publications that users recognise and abide by the legal requirements associated with these

- Users may download and print one copy of any publication from the public portal for the purpose of private study or research.

- You may not further distribute the material or use it for any profit-making activity or commercial gain

If the publication is distributed under the terms of Article 25fa of the Dutch Copyright Act, indicated by the "Taverne" license above, 


\section{Pleiotropic effects of non-structural matrix proteins in the stressed heart}

ECM remodeling in cardiotoxicity, aging and cardiac allograft rejection 
(c) copyright, G.C. van Almen, Maastricht 2012

All rights reserved. No part of this publication may be reproduced in any form or by any means, electronically, mechanically, by print, or otherwise, without written permission of the copyright owner.

\section{ISBN 978-94-6159-138-8}

Cover design by V.E. van Almen

Layout and printing: Datawyse | Universitaire Pers Maastricht 


\section{Pleiotropic effects of non-structural matrix proteins in the stressed heart}

ECM remodeling in cardiotoxicity, aging and cardiac allograft rejection

PROEFSCHRIFT

ter verkrijging van de graad van doctor

aan de Universiteit Maastricht,

op gezag van de Rector Magnificus,

Prof. Mr. G.P.M.F. Mols

volgens het besluit van het College van Decanen,

in het openbaar te verdedigen

op woensdag 16 mei 2012 om 14.00 uur

door

Gerardus Cornelis van Almen

Geboren op 13 april 1983 te Eindhoven

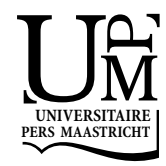




\section{Promotor}

Prof. dr. S. Heymans

\section{Copromotor}

Dr. B.L.M. Schroen

\section{Beoordelingscommissie}

Prof. dr. H.P. Brunner-La Rocca (voorzitter)

Dr. M. van Bilsen

Prof. dr. H. ten Cate

Prof. dr. R. Lijnen (KU Leuven)

Prof. dr. P.A.J. Schrauwen

The research described in this thesis was supported by a grant of the Dutch Heart Foundation (DHF2005B082).

Financial support by the Dutch Heart Foundation for the publication of this thesis is gratefully acknowledged.

gesubsidieerd door 


\section{Contents}

$\begin{array}{lll}\text { Chapter } 1 \text { General introduction } & 7\end{array}$

Chapter 2 Thrombospondins in the heart: potential functions in cardiac 17 remodeling

Chapter 3 The absence of thrombospondin-2 causes age-related dilated cardiomyopathy

Chapter 4 Absence of thrombospondin-2 increases cardiomyocyte damage and matrix disruption in doxorubicin-induced cardiomyopathy

Chapter 5 MicroRNA-18 and -19 regulate CTGF and TSP-1 expression in agerelated heart failure

Chapter 6 Matricellular proteins and matrix metalloproteinases mark the inflammatory and fibrotic response in human cardiac allograft rejection

Chapter 7 General discussion and conclusions

Summary

Samenvatting

Dankwoord

List of publications

Curriculum Vitae 

Chapter 1

General introduction 
Cardiovascular diseases (CVD) are the number one cause of death in Europe and accounts for $>2$ million deaths each year. With 41000 deaths in 2008 in the Netherlands, CVD comprised $30 \%$ of the total mortality in that year, with heart failure (HF) being the $3^{\text {rd }}$ cause after ischemic disease and stroke. ${ }^{1}$ Approximately 180.000 people in the Netherlands suffer from heart failure ${ }^{2}$, and the incidence grows with 35.000 new patients each year ${ }^{3}$, of which the majority is above the age of $75 .{ }^{4}$ Health care costs related to heart failure amounted 375 million euro in 2003 in the Netherlands. ${ }^{5}$ Although its contribution to overall mortality has decreased over the past 10 years, the incidence of heart failure will increase in the next decade due to the growing population of elderly and the increased survival rate after myocardial infarction. As the socioeconomic burden of heart failure is growing, the need for better prevention and treatment programs is increasing. In order to improve and identify new therapeutic strategies, we need to increase our understanding of the underlying mechanisms that contribute to the development of heart failure. In this thesis we address the pleiotropic role of the extracellular matrix (ECM) in heart failure. Although increasing evidence implicates a role for matrix proteins in modulating cell function, the majority of studies mainly addresses their ability to orchestrate the organization of collagens in the ECM. In contrast, this thesis proposes the ECM as a dynamic, plastic structure, that not solely provides a structural meshwork in which cardiac cells are embedded, but is also actively involved in regulating cell function and behavior in the healthy and diseased heart.

\section{Cardiac function and adaptation}

The healthy heart functions to pump blood throughout the body in order to provide peripheral organs with oxygen. Many mechanisms influence the rate with which the heart pumps out the blood, thereby enabling the heart to comply to different situations that alter the demand of oxygen throughout the body, such as exercise. The ability of the myocardium to adapt to different physiological conditions is often beneficial. However, if these conditions sustain or become of pathophysiological nature, the response changes from adaptive to maladaptive, resulting in cardiac failure. These pathophysiological conditions include an increased workload, often caused by high blood pressure, atherosclerosis, loss of cardiomyocytes following ischemic injury (myocardial infarction) or cardiotoxicity. Regardless of the origin of the condition, the heart responds by changing its size, shape and structure/composition. ${ }^{6,7}$ The process that underlies these alterations is generally termed cardiac remodeling, and involves a substantial reorganization of the cellular and extracellular matrix compartment of the myocardium. $^{8}$ In order to maintain the balance between adaptive and maladaptive remodeling, proper communication between cardiac cells and their surrounding matrix is indispensable. 
Cardiac remodeling: the interplay between the extracellular matrix and cardiac cells

Cell-matrix communication depends on the type of cells and the ECM components present in the heart, and thus will be affected when the heart changes its structure and composition during remodeling. The heart is made up of a cellular and ECM compartment. In terms of cell number, approximately one third of the cellular compartment consists of cardiomyocytes, whereas the rest includes cardiac fibroblasts, endothelial cells and vascular smooth muscle cells. The ECM is composed of structural and non-structural, matricellular, proteins. As the majority of the ECM consists of structural proteins such as elastin and the tensile collagen type I (approximately $90 \%$ of the collagen) and the more elastic collagen type III (approximately $10 \%$ of the collagen) ${ }^{9,10}$, the extracellular matrix is originally seen as the 'cement' of the heart, providing an architectural backbone that determines the strength of the cardiac tissue. Increasing evidence shows an important role for non-structural matricellular proteins in modulating cellular behavior by regulating cell shape, proliferation, migration and differentiation, but also maintenance of the extracellular environment through regulation of the ECM turnover via interactions with matrix metalloproteinases (MMPs) and their inhibitors (TIMPs). ${ }^{11}$ This has significantly changed the traditional view, that proposes a rather structural function of the ECM, into the ECM being a dynamic meshwork that provides a scaffold for cell-matrix interactions and thereby determines cell and tissue function.

\section{Cell-matrix communication: matricellular proteins modulate cardiomyocyte behavior}

In normal tissue homeostasis, the regulatory effect of matricellular proteins becomes apparent during embryonic development when their expression is high. During postnatal life basal expression of matricellular proteins is low. However, during episodes of injury they are re-expressed. ${ }^{8}$ In the heart, re-expression of matricellular proteins like osteonectin/SPARC ${ }^{12}$, syndecan- $1^{13}, \mathrm{TSP}^{1} \mathbf{1}^{14}, \mathrm{TSP}-2^{15}$, osteopontin ${ }^{16,17}$ and periostin ${ }^{18}$ protects against pomp failure.

Matricellular proteins are generally believed to be produced by fibroblasts, endothelial cells and inflammatory cells that have infiltrated the injured area. Locally expressed matricellular proteins regulate processes such as angiogenesis and fibrosis through modulation of migration and differentiation of endothelial and vascular smooth muscle cells and proliferation of fibroblasts. An increasing number of studies show that cardiomyocytes also secrete matricellular proteins, i.e. after myocardial infarction. ${ }^{14,19-21}$ Matricellular proteins bind various membrane receptors such as integrins, and thereby directly link the extracellular environment to the cardiomyocyte cytoskeleton and intracellular signaling pathways. ${ }^{11}$ Two family members, CTGF and periostin, even actively induce hypertrophy of cardiomyocytes. ${ }^{21,22}$ In addition, periostin was found to modulate PI3K-Akt signaling in cardiomyocytes via binding to surface integrins, triggering reentry of the cell cycle. ${ }^{23}$ TSP-2 also mediates Akt-survival signal- 
ing in cardiomyocytes, and in its absence, mice exhibit increased cardiomyocyte dropout and accelerated development of age-associated cardiomyopathy. ${ }^{20}$

\section{The thrombospondins}

Among the matricellular proteins, thrombospondins are highlighted as key-molecules during tumor formation, wound healing and angiogenesis, processes that are associated with significant remodeling of the ECM (reviewed $\mathrm{in}^{24}$ ). The family of thrombospondins (TSPs) consists of five members and can be distinguished in two subgroups based on their protein structure: the trimeric thrombospondin-1 (TSP-1) and thrombospondin-2 (TSP-2) and the pentameric thrombospondin-3 (TSP-3), thrombospondin4 (TSP-4) and thrombospondin-5 (TSP-5). Their different protein domains allow them to interact with a variety of ligands through which they affect different processes inand outside the cell. In addition to the shared type-II and type-III repeats, TSP-1 and TSP-2 contain type-I repeats (TSR) which are lacking in TSP-3, TSP-4 and TSP-5. These type-I repeats are implicated in binding to cell surface receptors CD36 and $\beta 1$-integrin, TGF- $\beta 1$, and MMPs, and link TSP-1 and TSP- 2 to remodeling associated processes such as cell migration, differentiation, inflammation, angiogenesis, and fibrosis (reviewed $\mathrm{in}^{25}$ ). The functions of TSP-1 and TSP-2 are extensively studied in models of wound healing and tumor formation, which all point towards a regulatory role in ECM remodeling. ${ }^{26}$ However, the exact functions of TSP-1 and TSP-2 seem to be highly dependent on their spatiotemporal expression and the availability of their natural bindingpartners.

Increased TSP-1, TSP-2, TSP-3, and TSP-4 levels have been reported after cardiac injury. ${ }^{15,27}$ In concordance, enhanced TSP-2 expression was associated with hypertensive heart disease and cardiac aging and locally expressed TSP-1 controls the inflammatory response after $\mathrm{MI}$ to prevent expansion of the infarcted area. ${ }^{14,15}$ In vascular remodeling and wound repair, TSP-1 and TSP-2 have also been implicated in antiangiogenic nitric oxide signaling and the regulation of TGF- $\beta 1$ activity. Together, increasing evidence suggests a role for TSP-1 and TSP-2 in regulating cell function and shaping of the extracellular landscape following cardiac injury. Chapter 2 reviews the biological role of TSPs in the ischemic and hypertensive heart.

\section{Aims and outline of the thesis}

Cardiac remodeling occurs in response to either mechanical overload or loss of contractile capacity, such as in hypertension and myocardial infarction, which are the most common pathological conditions leading to heart failure. ${ }^{28}$ It is for this reason, that most research regarding the role of matricellular proteins in regulating cardiac adaptation, is performed in view of the hypertrophic and ischemic heart. Nevertheless, cardiotoxicity, aging, and cardiac allograft rejection are also associated with loss of car- 
diomyocytes and remodeling. However, the role of matricellular proteins, and TSPs in particular, is less well understood during these pathological conditions. The aim of this thesis is to extend the common knowledge on matricellular proteins in the hypertrophic and ischemic heart, towards their function in cardiotoxicity, aging and cardiac allograft rejection. The thesis focuses on their ability to regulate ECM integrity and cardiomyocyte function during cardiotoxicity, and the changes in expression profile related to functional decline with aging and the grade of rejection after cardiac allograft transplantation. Beside the matricellular proteins TSP-1 and TSP-2, also connective tissue growth factor (CTGF), the matrix proteoglycan syndecan-1 and matrix metalloproteinase 9 are discussed.

\section{Implications for thrombospondin-2 in myocardial aging and doxorubicin-induced cardiotoxicity}

Chapter 3 discusses the role of TSP-2 in age-related cardiac remodeling and for the first time addresses the bifunctionality of this matricellular protein in the heart. Absence of TSP-2 accelerates the onset of an age-related decline in cardiac function which is accompanied by ventricular dilation and fibrosis. Additional to the effect on the ECM environment, TSP-2 also affects the cellular compartment as its absence results in an increased loss of cardiomyocytes. These pleiotropic functions were further evaluated in a model of doxorubicin (DOX)-induced cardiotoxicity (chapter 4). The antineoplastic drug doxorubicin is successfully used in treatment of various cancers, however its clinical application is limited because of its cardiotoxic side effects. Oxidative stress is recognized as the primary cause of DOX-induced cardiotoxicity, as it targets vital signaling pathways within the cardiomyocyte and compromises the integrity of their surrounding matrix. As previous studies showed that TSP-2 is implicated in ECM turnover and cardiomyocyte survival signaling in hypertensive heart disease and aging, we hypothesized that this matrix molecule has a protective role against the deleterious effects of DOX on the heart. This study for the first time implicates a role for a matrix element in doxorubicin-associated cardiomyopathy, and shows that the absence of TSP-2 causes increased damage of cardiomyocytes and disruption of the ECM after chronic DOX treatment. The pleiotropic nature of TSP-2 is illustrated by the finding that it modulates both cellular and matrix responses to cardiotoxicity as impaired Akt signaling in cardiomyocytes together with enhanced MMP-2 activity contributed to cardiac injury and dysfunction in TSP-2-KO mice after DOX.

\section{Regulation of matricellular protein expression by microRNAs}

The fact that matricellular proteins are generally expressed at high levels during embryonic development and low levels during postnatal live suggests involvement of an intrinsic mechanism that regulates their expression. In acute cardiac injury, the release of cytokines and growth factors such VEGF, EGF and TGF- $\beta$ is recognized as an impor- 
tant stimulus that controls the expression of various matricellular proteins. Studies on other levels of transcriptional control such as epigenetic events and regulation by transcription factors are scarce. Hence, SPARC expression is regulated via the transcription factor Brg-1 in mammary carcinomas and methylation of its promoter site has been

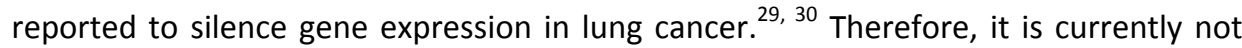
understood whether growth and transcription factors or epigenetic control also contribute to the regulation of matricellular proteins during postnatal live when expression levels are relatively low.

The discovery of small non-coding RNA sequences, termed microRNAs (miRNAs), that regulate gene expression through complementary binding to their specific target mRNA, has opened new doors to study the regulation of gene expression. This highly conserved mechanism has been shown to regulate embryonic development, tumorgenesis, and importantly, was shown to be involved in various pathological cardiac conditions as well. Chapter 5 describes how age-related changes in microRNA-17 92 expression, in particular that of the cluster members miR-18 and miR-19, regulate CTGF and TSP-1 levels and contribute to ECM remodeling associated with cardiac aging and failure. Cardiac aging involves the functional decline of the heart caused by cardiomyocyte loss, left-ventricular hypertrophy, dilatation, and accumulation of ECM. ${ }^{31}$ The constant wear and tear in the aging heart induces changes in gene expression that give rise to an adaptive remodeling response that defines the phenotypic characteristics of the aged myocardium. ${ }^{32,33}$ As often reported in the hypertrophic and ischemic heart, with aging expression of various matricellular proteins re-appears or increases. Moreover, mice deficient for TSP-2 age poorly and cardiomyocyte-specific overexpression of CTGF accelerates the onset of age-associated cardiomyopathy in mice. ${ }^{20,22}$ Despite the aging-specific gene-expression profiles identified in aged hearts and cardiomyocytes, the underlying mechanisms that controls this age-associated gene pattern remains elusive. Now, regulation by miRNAs has emerged as a novel mechanism that modulates geneexpression and fine-tunes protein levels in a post-transcriptional manner. This study focused on the age-related expression profile of the miR-17 92 cluster, which has been shown to be down regulated in aging and cellular senescence ${ }^{34,35}$, and, importantly, is known to regulate CTGF and TSP- $1^{36}$, which are both increased in the injured heart. ${ }^{14,37}$ Our findings suggest that upregulation of the cluster members miR-18 and miR-19 in the aged failure-protected heart blunts the expression of TSP-1 and CTGF in order to dampen the fibrotic remodeling process that contributes to the functional decline with age. Moreover, these findings suggest that regulation of matricellular protein expression by miRNAs is a new level of studying cardiac remodeling.

Matricellular proteins in cardiac allograft rejection: implications in inflammation and fibrosis

Chapter 6 describes the expression of matricellular proteins, proteoglycans, MMPs, and TIMPs relative to the degree of cardiac allograft rejection (CAR) following heart 
transplantation. CAR is characterized by severe cardiac inflammation and remodeling, and results in detrimental left ventricular dysfunction. ${ }^{38}$ The tightly regulated remodeling response is orchestrated by the interplay between matricellular proteins, MMPs and their inhibitors (TIMPS). Cardiac inflammation is a major hallmark of rejection after transplantation. Importantly, MMPs, TIMPs and matricellular proteins have been implicated in modulating inflammation, ECM remodeling and cell behavior in the heart. ${ }^{11,}$ ${ }^{38-40}$ Our study indicated a clear correlation between cardiac mRNA levels of syndecan1 and MMP-9 with the inflammatory markers TNF-a and IL-6 and revealed that elevated expression of these matrix elements strongly relates to the degree of CAR. Analysis of protein expression, further substantiated the potential of syndecan- 1 and MMP-9 expression as indicators of CAR, by showing that the expression parallels the progressive increase in T-cells and macrophages. Together, our data point towards syndecan-1 and MMP-9 as important factors that contribute to adverse cardiac inflammation and subsequent cardiac remodeling process in human CAR.

\section{Concluding remarks}

Although their role in left-ventricular hypertrophy and ischemia is well established, this thesis implicates matricellular proteins in cardiotoxicity, cardiac aging, and cardiac allograft transplantation. The findings in this thesis demonstrate the multiple functions of these proteins in the heart, showing the ability of matricellular proteins to regulate cardiomyocyte function and ECM integrity in doxorubicin-induced cardiotoxicity, ageassociated remodeling and inflammation associated with CAR. The versatility of roles of matricellular proteins provided by these studies illustrates their pleiotropic nature and strengthens the idea of the ECM as a dynamic meshwork that provides a scaffold for cell-matrix interactions and thereby determines cardiac cell and tissue function under pathological conditions. As key-modulators of ECM integrity and cell function in the heart, matricellular proteins form a large group potential targets that can be used to orchestrate the remodeling process, and pave the way towards discovery of new therapeutic strategies against the development of heart failure. 


\section{References}

1 Vaartjes I, Van Dis I, Visseren FL, Bots ML. Hart- en Vaatziekten in Nederland, in Hart- en vaatziekten in Nederland 2009, cijfers over leefstijl- en risicofactoren, ziekte en sterfte. Nederlandse Hartstichting: Den Haag; 2009.

2 Hoes AW, Mosterd A, Rutten FH, Poos MJJC. Hoe vaak komt hartfalen voor en hoeveel mensen sterven eraan? In: Volksgezondheid Toekomst Verkenning, Nationaal Kompas Volksgezondheid. RIVM: Bilthoven; 2006.

3 Vaartjes I, Peters RJG, Van Dis SJ, Bots ML. Hart- en vaatziekten in Nederland 2007, cijfers over leefstijlen risicofactoren, ziekte en sterfte. Nederlandse Hartstichting: Den Haag; 2007.

4 Cost B. Heart failure in the elderly (PhD thesis). Erasmus Universiteit: Rotterdam; 2000.

5 Slobbe LCJ, Kommer GJ, Smit JM, Groen J, Meerding WJ, Polder JJ. Kosten van Ziekten in Nederland 2003; Zorg in euro's. RIVM: Bilthoven; 2006.

6 Cohn JN. Critical review of heart failure: the role of left ventricular remodeling in the therapeutic response. Clin Cardiol. 1995;18(9 Suppl 4):IV4-12.

7 Cohn JN. Structural basis for heart failure. Ventricular remodeling and its pharmacological inhibition. Circulation. 1995;91(10):2504-2507.

8 Swynghedauw B. Molecular mechanisms of myocardial remodeling. Physiol Rev. 1999;79(1):215-262.

9 Brown RD, Ambler SK, Mitchell MD, Long CS. The cardiac fibroblast: therapeutic target in myocardial remodeling and failure. Annu Rev Pharmacol Toxicol. 2005;45:657-687.

10 Fedak PW, Verma S, Weisel RD, Li RK. Cardiac remodeling and failure From molecules to man (Part II). Cardiovasc Pathol. 2005;14(2):49-60.

11 Spinale FG. Myocardial matrix remodeling and the matrix metalloproteinases: influence on cardiac form and function. Physiol Rev. 2007;87(4):1285-1342.

12 Schellings MW, Vanhoutte D, Swinnen M, Cleutjens JP, Debets J, van Leeuwen RE, d'Hooge J, Van de Werf F, Carmeliet P, Pinto YM, Sage EH, Heymans S. Absence of SPARC results in increased cardiac rupture and dysfunction after acute myocardial infarction. J Exp Med. 2009;206(1):113-123.

13 Vanhoutte D, Schellings MW, Gotte M, Swinnen M, Herias V, Wild MK, Vestweber D, Chorianopoulos E, Cortes V, Rigotti A, Stepp MA, Van de Werf F, Carmeliet P, Pinto YM, Heymans S. Increased expression of syndecan-1 protects against cardiac dilatation and dysfunction after myocardial infarction. Circulation. 2007;115(4):475-482.

14 Frangogiannis NG, Ren G, Dewald O, Zymek P, Haudek S, Koerting A, Winkelmann K, Michael LH, Lawler J, Entman ML. Critical role of endogenous thrombospondin-1 in preventing expansion of healing myocardial infarcts. Circulation. 2005;111(22):2935-2942.

15 Schroen B, Heymans S, Sharma U, Blankesteijn WM, Pokharel S, Cleutjens JP, Porter JG, Evelo CT, Duisters R, van Leeuwen RE, Janssen BJ, Debets JJ, Smits JF, Daemen MJ, Crijns HJ, Bornstein P, Pinto YM. Thrombospondin-2 is essential for myocardial matrix integrity: increased expression identifies failureprone cardiac hypertrophy. Circ Res. 2004;95(5):515-522.

16 Collins AR, Schnee J, Wang W, Kim S, Fishbein MC, Bruemmer D, Law RE, Nicholas S, Ross RS, Hsueh WA. Osteopontin modulates angiotensin II-induced fibrosis in the intact murine heart. J Am Coll Cardiol. 2004;43(9):1698-1705.

17 Trueblood NA, Xie Z, Communal C, Sam F, Ngoy S, Liaw L, Jenkins AW, Wang J, Sawyer DB, Bing $O H$, Apstein CS, Colucci WS, Singh K. Exaggerated left ventricular dilation and reduced collagen deposition after myocardial infarction in mice lacking osteopontin. Circ Res. 2001;88(10):1080-1087.

18 Shimazaki M, Nakamura K, Kii I, Kashima T, Amizuka N, Li M, Saito M, Fukuda K, Nishiyama T, Kitajima S, Saga Y, Fukayama M, Sata M, Kudo A. Periostin is essential for cardiac healing after acute myocardial infarction. J Exp Med. 2008;205(2):295-303.

19 Kemp TJ, Aggeli IK, Sugden PH, Clerk A. Phenylephrine and endothelin-1 upregulate connective tissue growth factor in neonatal rat cardiac myocytes. J Mol Cell Cardiol. 2004;37(2):603-606. 
20 Swinnen M, Vanhoutte D, Van Almen GC, Hamdani N, Schellings MW, D'Hooge J, Van der Velden J, Weaver MS, Sage EH, Bornstein P, Verheyen FK, VandenDriessche T, Chuah MK, Westermann D, Paulus WJ, Van de Werf F, Schroen B, Carmeliet P, Pinto YM, Heymans S. Absence of thrombospondin-2 causes age-related dilated cardiomyopathy. Circulation. 2009;120(16):1585-1597.

21 Oka T, Xu J, Kaiser RA, Melendez J, Hambleton M, Sargent MA, Lorts A, Brunskill EW, Dorn GW, 2nd, Conway SJ, Aronow BJ, Robbins J, Molkentin JD. Genetic manipulation of periostin expression reveals a role in cardiac hypertrophy and ventricular remodeling. Circ Res. 2007;101(3):313-321.

22 Panek AN, Posch MG, Alenina N, Ghadge SK, Erdmann B, Popova E, Perrot A, Geier C, Dietz R, Morano I, Bader M, Ozcelik C. Connective tissue growth factor overexpression in cardiomyocytes promotes cardiac hypertrophy and protection against pressure overload. PLoS One. 2009;4(8):e6743.

23 Kuhn B, del Monte F, Hajjar RJ, Chang YS, Lebeche D, Arab S, Keating MT. Periostin induces proliferation of differentiated cardiomyocytes and promotes cardiac repair. Nat Med. 2007;13(8):962-969.

24 Tan K, Lawler J. The interaction of Thrombospondins with extracellular matrix proteins. J Cell Commun Signal. 2009;3(3-4):177-187.

25 Bornstein P. Thrombospondins function as regulators of angiogenesis. J Cell Commun Signal. 2009;3(34):189-200.

26 Kyriakides TR, Tam JW, Bornstein P. Accelerated wound healing in mice with a disruption of the thrombospondin 2 gene. J Invest Dermatol. 1999;113(5):782-787.

27 Rysa J, Leskinen $\mathrm{H}$, Ilves $\mathrm{M}$, Ruskoaho $\mathrm{H}$. Distinct upregulation of extracellular matrix genes in transition from hypertrophy to hypertensive heart failure. Hypertension. 2005;45(5):927-933.

28 Swynghedauw B. Phenotypic plasticity of adult myocardium: molecular mechanisms. J Exp Biol. 2006;209(Pt 12):2320-2327.

29 Suzuki M, Hao C, Takahashi T, Shigematsu H, Shivapurkar N, Sathyanarayana UG, lizasa T, Fujisawa T, Hiroshima K, Gazdar AF. Aberrant methylation of SPARC in human lung cancers. Br J Cancer. 2005;92(5):942-948.

30 Xu YZ, Heravi M, Thuraisingam T, Di Marco S, Muanza T, Radzioch D. Brg-1 mediates the constitutive and fenretinide-induced expression of SPARC in mammary carcinoma cells via its interaction with transcription factor Sp1. Mol Cancer. 2010;9:210.

31 Lakatta EG, Sollott SJ. Perspectives on mammalian cardiovascular aging: humans to molecules. Comp Biochem Physiol A Mol Integr Physiol. 2002;132(4):699-721.

32 Bodyak N, Kang PM, Hiromura M, Sulijoadikusumo I, Horikoshi N, Khrapko K, Usheva A. Gene expression profiling of the aging mouse cardiac myocytes. Nucleic Acids Res. 2002;30(17):3788-3794.

33 Park SK, Prolla TA. Gene expression profiling studies of aging in cardiac and skeletal muscles. Cardiovasc Res. 2005;66(2):205-212.

34 Hackl M, Brunner S, Fortschegger K, Schreiner C, Micutkova L, Muck C, Laschober GT, Lepperdinger G, Sampson N, Berger P, Herndler-Brandstetter D, Wieser M, Kuhnel H, Strasser A, Rinnerthaler M, Breitenbach M, Mildner M, Eckhart L, Tschachler E, Trost A, Bauer JW, Papak C, Trajanoski Z, Scheideler M, Grillari-Voglauer R, Grubeck-Loebenstein B, Jansen-Durr P, Grillari J. miR-17, miR-19b, miR-20a, and miR106a are down-regulated in human aging. Aging Cell.9(2):291-296.

35 Li G, Luna C, Qiu J, Epstein DL, Gonzalez P. Alterations in microRNA expression in stress-induced cellular senescence. Mech Ageing Dev. 2009;130(11-12):731-741.

36 Dews M, Homayouni A, Yu D, Murphy D, Sevignani C, Wentzel E, Furth EE, Lee WM, Enders GH, Mendell JT, Thomas-Tikhonenko A. Augmentation of tumor angiogenesis by a Myc-activated microRNA cluster. Nat Genet. 2006;38(9):1060-1065.

37 Chen MM, Lam A, Abraham JA, Schreiner GF, Joly AH. CTGF expression is induced by TGF- beta in cardiac fibroblasts and cardiac myocytes: a potential role in heart fibrosis. J Mol Cell Cardiol. 2000;32(10):18051819.

38 Lechler RI, Garden OA, Turka LA. The complementary roles of deletion and regulation in transplantation tolerance. Nat Rev Immunol. 2003;3(2):147-158.

39 Vanhoutte D, Schellings M, Pinto Y, Heymans S. Relevance of matrix metalloproteinases and their inhibitors after myocardial infarction: a temporal and spatial window. Cardiovasc Res. 2006;69(3):604-613. 
40 Vanhoutte D, Heymans S. TIMPs and cardiac remodeling: 'Embracing the MMP-independent-side of the family'. J Mol Cell Cardiol. 2009. 


\section{Chapter 2}

\section{Thrombospondins in the heart: potential functions in cardiac remodeling}

Mark W.M. Schellings*, Geert C. van Almen*, E. Helene Sage, Stephane Heymans *These authors contributed equally to this manuscript

Modified from Journal of Cell Communication and Signaling. 2009 Dec;3(3-4):201-213 


\begin{abstract}
Cardiac remodeling after myocardial injury involves inflammation, angiogenesis, left ventricular hypertrophy and matrix remodeling. Thrombospondins (TSPs) belong to the group of matricellular proteins, which are non-structural extracellular matrix proteins that modulate cell-matrix interactions and cell function in injured tissues or tumors. They interact with different matrix and membrane-bound proteins due to their diverse functional domains. That the expression of TSPs strongly increases during cardiac stress or injury indicates an important role for them during cardiac remodeling. Recently, the protective properties of TSP expression against heart failure have been acknowledged. The current review will focus on the biological role of TSPs in the ischemic and hypertensive heart, and will describe the functional consequences of TSP polymorphisms in cardiac disease.
\end{abstract}




\section{Introduction}

Heart failure (HF) is a condition resulting from different cardiovascular diseases and is one of the leading causes of mortality in Western society. ${ }^{1-3}$ Acute myocardial infarction (MI), hypertensive heart disease, myocarditis, and genetic cardiomyopathies are diseases that involve extensive cardiac remodeling, and often result in an increased risk of developing HF. Cardiac dysfunction after injury or stress is the outcome of a complex interplay between environmental and mechanical forces, different cell types, and various molecular processes that determine cell behavior. In vivo and in vitro studies have identified different mechanisms like the renin-angiotensin system (RAS) ${ }^{4}$, integrin-signaling ${ }^{5,6}$, and matrix remodeling ${ }^{7}$, among many others implicated in the progression of HF.

The extracellular matrix (ECM) is a crucial system for maintaining strength and organization of the cardiac tissue, and cardiac cell communication. The ECM forms a complex meshwork composed of structural proteins (such as type I and III collagen), proteoglycans, glycosaminoglycans, and a basement membrane, which together are responsible for tissue strength and structure. Matricellular proteins are non-structural proteins with a minimal expression in the normal heart. However, their expression increases after injury, where they modulate cell function and behavior through regulation of cell-cell and cell-matrix interactions. ${ }^{7}$

Alteration in ventricular shape, size and function take place during cardiac injury, a process termed remodeling [reviewed $\mathrm{in}^{7,8}$ ]. Matricellular proteins appear to be key players during cardiac remodeling. ${ }^{9}$ Animal experiments revealed that the matricellular proteins thrombospondin 1 (TSP- 1$)^{10}$, thrombospondin 2 (TSP-2) $)^{11}$, osteopontin ${ }^{12,13}$, periostin $^{14}$, and SPARC ${ }^{15}$ all protect the heart from developing pump failure. Among the matricellular proteins, thrombospondins (TSPs) were highlighted as important players in the process of cardiac remodeling. ${ }^{16}$ This review summarizes our current knowledge of the role of TSPS, in particular TSP-1 and TSP-2, in cardiac remodeling during hypertrophy and after MI (Table 1). It will elaborate the molecular characteristics of TSPs that may relate to their role in preventing HF (Figures 1 and 2).

Table 1. Expression of TSPs and cardiac phenotypes of different TSP null mice in cardiac pathologies.

\begin{tabular}{|c|c|c|c|c|c|}
\hline & \multicolumn{2}{|l|}{ Expression } & \multicolumn{2}{|c|}{ TSP null cardiac phenotype } & \multirow[t]{2}{*}{ references } \\
\hline & $\begin{array}{l}\text { Pressure } \\
\text { overload }\end{array}$ & $\mathrm{Ml}$ & Pressure overload & $\mathrm{Ml}$ & \\
\hline TSP-1 & Increased & $\begin{array}{l}\text { Increased } \\
\text { during early } \\
\text { phase }\end{array}$ & ND & $\begin{array}{l}\text { Increased inflammatory } \\
\text { response; More extensive } \\
\text { postinfarct remodeling }\end{array}$ & $10,11,21,22$ \\
\hline TSP-2 & Increased & ND & $\begin{array}{l}\text { Severe cardiac failure } \\
\text { and cardiac rupture }\end{array}$ & $\begin{array}{l}>90 \% \text { Cardiac rupture } \\
\text { within } 3 \text { days }\end{array}$ & 11,100 \\
\hline TSP-3 & Increased & ND & ND & ND & 11 \\
\hline TSP-4 & Increased & ND & ND & ND & $23,24,25$ \\
\hline
\end{tabular}




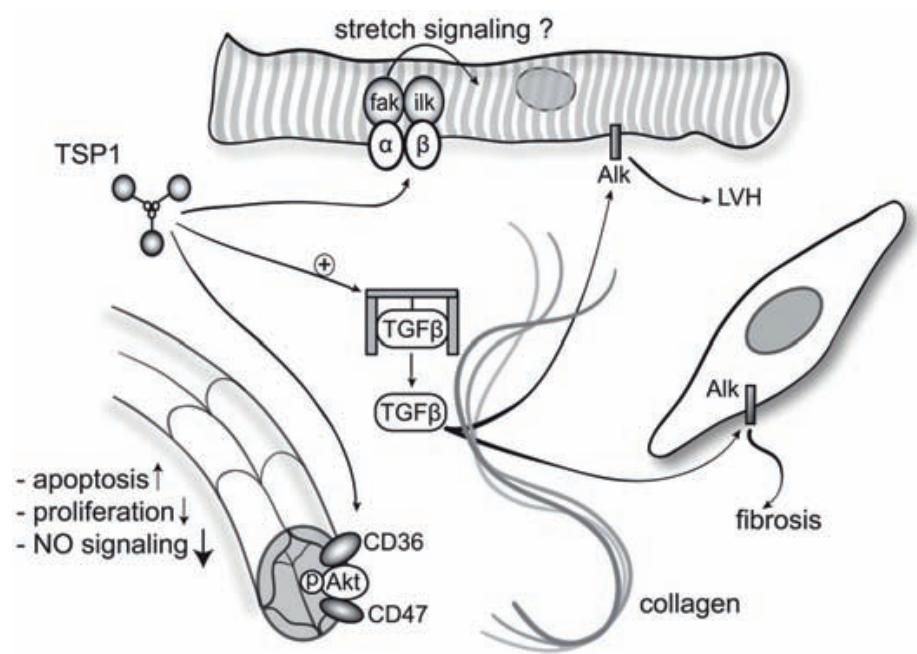

Figure 1. Potential functions of TSP-1 in cardiac remodeling during pressure overload or after MI. TSP-1 is an inhibitor of angiogenesis and an activator of TGF- $\beta_{1}$, and thereby influences the behavior of endothelial cells, fibroblasts and myocytes. By binding to integrins, TSP-1 might also alter the adhesive or stretch-induced signaling of cells. $\alpha$ indicates $\alpha$-integrin subunit, $\beta$ indicates $\beta$-integrin subunit, fak: focal adhesion kinase; ilk: integrin-linked kinase; Alk: activin-like kinase; LVH: left ventricular hypertrophy; TGF: transforming growth factor.

\section{Thrombospondins in cardiac homeostasis}

Although the expression of thrombospondins is low in the normal, unstressed heart, recent evidence suggests that they are important in modulating physiological angiogenesis and blood pressure. ${ }^{17,}{ }^{18}$ Global deletion of TSP-1 resulted in significant increases in cardiac and skeletal muscle capillarity, which was associated with increased basal levels of VEGF in TSP-1-null mice. ${ }^{19}$ Together with the effects of TSP-1 on endothelial cell activity, this finding indicates that TSP-1 is important in regulating physiological angiogenesis. The influence of TSP-2 on physiological angiogenesis was demonstrated in a three-dimensional angiogenesis assay in which TSP-2 limited angiogenesis by decreasing gelatinolytic activity, and by accelerated recovery of blood flow in TSP-2null mice after hind limb ischemia. Also, increased endothelial cell organization and migration was observed when these cells were cultured on TSP-2-null ECM. Thus, TSP2 can inhibit physiological angiogenesis by limiting the gelatinolytic activity of endothelial cells and by its influence on ECM structure. In addition to the effects of TSPs on physiological angiogenesis, recent evidence suggests that TSP-1 is able to modulate nitric oxide (NO) controlled blood pressure via its receptor CD47/integrin associated protein (CD47/IAP). ${ }^{17}$ 


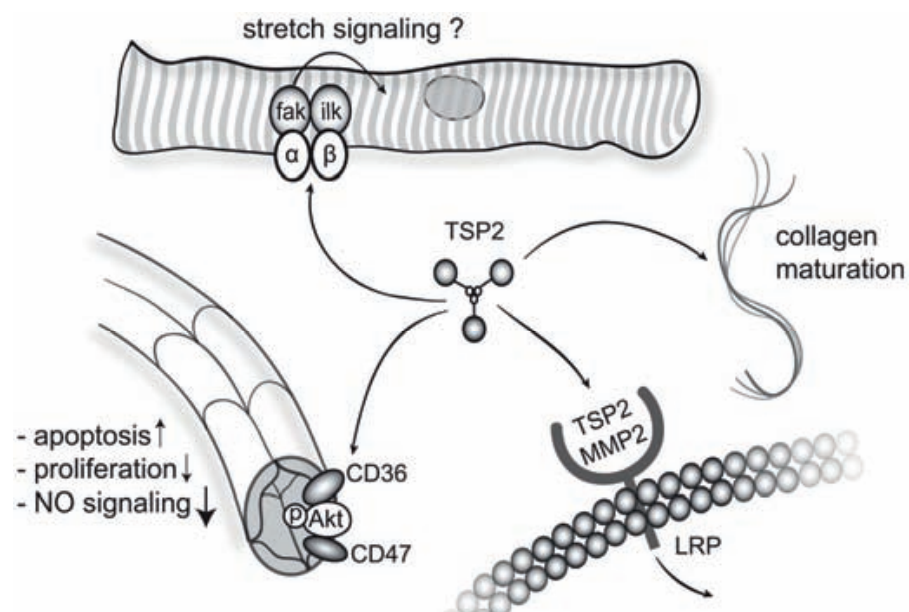

Figure 2. Potential functions of TSP-2 in cardiac remodeling. Together with TSP-1, TSP-2 is an inhibitor of angiogenesis, and through integrin-binding TSP-2 might also influence adhesive or stretch-induced signaling. An important feature of TSP-2 is its capacity to bind pro- or active MMP-2, resulting in endocytosis via the LRP-receptor, and inhibition of its proteolytic activity. The influence of TSP-2 on collagen maturation has also been established. $\alpha$ indicates $\alpha$-integrin subunit, $\beta$ indicates $\beta$-integrin subunit, fak: focal adhesion kinase; ilk: integrin-linked kinase; MMP: matrix metalloproteinase; LRP: low density lipoprotein receptor.

\section{Thrombospondins in hypertrophic cardiac remodeling}

Cardiac hypertrophy, an increase in heart muscle mass, reflects an adaptation of the myocardium in response to increased mechanical stress or volume overload. It occurs as a physiological response, often seen in athletes, or as a pathologic one, which is associated with maladaptive changes. Persistent pathological hypertrophy finally evolves in cardiac dysfunction and clinical HF. Apart from mechanical stress, various growth factors and other stimuli can induce cellular signaling events that lead to hypertrophy. Although cardiac myocytes play an important role in the hypertrophic process, other cell types such as fibroblasts, endothelial cells, and smooth muscle cells may also affect the hypertrophic response. ${ }^{20}$

The ECM plays an important role in hypertrophic remodeling by providing architectural support to the cardiac tissue, and mediating cell-ECM communication. Here, the different mechanisms by which TSPs may affect hypertrophic cardiac remodeling will be discussed.

\section{Thrombospondin expression in cardiac hypertrophy}

Expression of TSP-1, TSP-2, and TSP-3 was increased during heart failure in a model of hypertensive hypertrophic remodeling ${ }^{11}$ (Table 1 ). The homozygous renin transgenic TGR(mRen2)27 rat suffers from severe hypertension that leads to cardiac hypertrophy 
at 10 weeks of age, with approximately $50 \%$ developing quick decompensation at $12-$ 14 weeks of age. Biopsies taken at 10 weeks of age allowed retrospective comparison of gene expression between the decompensated and compensated group. TSP-1, TSP2 , and TSP-3 transcript levels were all significantly enhanced at 10 weeks of age in the rats prone to develop quick decompensation. Moreover, TSP-2 transcript levels were also increased in patients with cardiac hypertrophy, secondary to aortic stenosis, and TSP-2 expression was particularly elevated in patients with a depressed ejection fraction $(<55 \%) .{ }^{11}$ Other in vivo studies demonstrated enhanced expression of TSP-1 after transverse aortic constriction and sustained alpha-adrenergic drive ${ }^{21,22}$ (Table 1). A study by Rysä et al. identified the pentameric, B-type TSP-4 to be increased in the hearts of spontaneously hypertensive rats (SHR) in comparison to age-matched Wistar Kyoto rats (WKY) ${ }^{23}$ (Table 1 ). The expression of TSP-4 transcripts gradually increased with age in the SHR, with the highest levels at 20 months of age. TSP-4 expression is also enhanced in response to acute pressure overload induced by angiotensin-II (AngII) and arginine-vasopressin (AVP) infusion ${ }^{24}$ (Table 1). Ang-II infusion resulted in a rapid increase in left ventricular (LV) TSP-4 mRNA levels, the highest at 6 hours after infusion. AVP infusion also increased TSP-4 expression, that was limited to LV endothelial cells. ${ }^{24}$ Furthermore, TSP-4 was upregulated at an early stage in response to cardiac overload, similar to TSP-1. ${ }^{24}$ TSP-4 expression also increased significantly in patients with end-stage dilated cardiomyopathy ${ }^{25}$ (Table 1). These studies indicate an important role for TSP-1, $-2,-3$, and -4 in left ventricular hypertrophic cardiac remodeling following pressure overload.

ECM remodeling during cardiac hypertrophy: thrombospondins and the MMP-TIMP balance

MMPs and TIMPs are actively involved in the process of hypertrophic cardiac remodeling. ${ }^{7}$ MMPs degrade ECM proteins and are divided into four classes: (1) collagenases, which include MMP-1, $-8,-13$, and $-18 ;(2)$ the gelatinases, which include MMP-2, and 9; (3) the stromelysins, including MMP-3, -10, and -11; and (4) the membrane-type MMPs (MT-MMPs), including MMP-14, $-17,-24$, and -25 . The TIMP family consists of 4 family members: TIMP1-4. In the uninjured heart, a balance between the synthesis and breakdown of ECM is maintained by MMPs and TIMPs ${ }^{7}$. During the transition of cardiac hypertrophy to heart failure, however, the balance shifts towards increased MMP activity, leading to myocyte slippage and ventricular dilatation.

TSP-1 and TSP-2 share a large overall similarity in their protein structure and therefore interact with the same ligands. Both TSP-1 and TSP-2 contain three different repeated sequence motifs (TSRs), known as type 1, type 2, and type 3 repeats. These TSRs allow TSP-1 and TSP- 2 to interact with MMPs, thereby regulating the activity of these proteinases. ${ }^{26,27}$ TSP-2-null skin fibroblasts have a defect in attachment to different matricellular proteins in vitro as a consequence of increased levels of active MMP$2 .^{26}$ In addition, the increased activity of MMP-2 causes enhanced proteolysis of cell- 
surface tissue transglutaminase (tTG), an enzyme important for collagen crosslinking. ${ }^{28}$ Later, it was demonstrated that TSP-2 is actively involved in the clearance of MMP-2 by directly targeting MMP-2 to form a complex that is endocytosed by the low density lipoprotein-related scavenger receptor (LRP1) ${ }^{29}$ (Figure 2). In a model of dermal wound healing, mice that lack TSP-2 showed aberrant ECM remodeling associated with increased levels of MMP-2, MMP-9, TIMP-1 and TIMP-2. ${ }^{30}$ In concordance, TSP-2null mice had increased MMP-2 activity after infusion of angiotensin II, contributing to an increased incidence of left ventricular rupture and dilatation. ${ }^{11}$

Whether TSP-1 may also regulate MMP activity is less certain, depending on celltype specific actions of TSP-1. It increases MMP-9 activity in endothelial cells and in gastric cancer $^{31,32}$, whereas others reported an inhibition of tumor growth by TSP-1 via its attenuation of MMP-9 activity. ${ }^{33}$ In vascular smooth muscle cells, TSP-1 induced MMP-2 activity $^{34}$, but the mechanism remains unclear. The contradictory results of TSP-1 on the regulation of MMP activation may depend on the opposite effects exerted by the different domains of TSP-1. Taraboletti et al. reported that, in rabbit corneal endothelial cells, the 25-kDa heparin-binding domain of TSP-1 increased MMP-2 and MMP-9 activity and decreased TIMP-2 levels, whereas the 140 kDa carboxyterminal fragment of TSP-1 upregulated TIMP-2 expression and inhibited MMP-2 and MMP-9 activities. ${ }^{35}$ Similar experiments with capillary endothelial cells isolated from bovine hearts confirmed these results. ${ }^{36}$ Reports on the influence of TSP-3, TSP-4, and TSP-5 on MMP activity are lacking.

\section{Thrombospondins and the renin-angiotensin system (RAS) in cardiac hypertrophy}

The RAS is activated in response to pressure overload of the heart, and contributes to the process of myocardial remodeling. ${ }^{4,37}$ The effector molecule of the RAS, angiotensin-II (Ang-II), triggers cell-specific responses in cardiac fibroblasts and myocytes through binding to the angiotensin type I receptor $\left(A T_{1} R\right) .{ }^{38-41}$ Binding of Ang-II to the $A T_{1} R$ induces synthesis of the locally secreted growth factor TGF- $\beta_{1}$ in cardiac fibroblasts and myocytes. Interaction of TGF- $\beta_{1}$ with its receptor causes expression of ECM proteins, collagen synthesis, and proliferation of cardiac fibroblasts. ${ }^{42-46}$ Tissue culture experiments showed that the paracrine release of TGF- $\beta_{1}$ from fibroblasts mediates Ang-II induced hypertrophy of cardiac myocytes as blocking antibodies to TGF- $\beta_{1}$ abolished myocyte growth. ${ }^{47}$ Also, blockade of the $A T_{1} R$ inhibits the expression of TGF- $\beta_{1}$ as well as the resulting cardiac hypertrophy and fibrosis, indicating a crucial link between Ang-II and TGF- $\beta_{1} .{ }^{48,49}$ Further support for the link between the RAS and TGF- $\beta_{1}$ is provided by studies that show upregulation of TGF- $\beta_{1}$ in patients with idiopathic hypertrophic cardiomyopathy and dilated cardiomyopathy ${ }^{49-55}$, and upregulation of angiotensin converting enzyme (ACE) and TGF- $\beta_{1}$ in pressure-overloaded human hearts is correlated with the degree of fibrosis. ${ }^{56}$

From the sequence similarity between TSP-1 and TSP-2, it could be expected that the binding partners of TSP-1 also interact with TSP-2. The type I repeats, which are 
characteristic for both TSP-1 and TSP-2, have been described in the activation of latent TGF- $\beta_{1}$ by TSP-1. ${ }^{57}$ The activation of TGF- $\beta_{1}$ links TSP- 1 to the process of hypertrophic cardiac remodeling (Figure 1). As indicated above, TSP-1 is increased in cardiac hypertrophy. Activation of latent TGF- $\beta_{1}$ involves direct interaction with two sequences in the type 1 repeats of TSP-1. The KRFK sequence in the first TSR activates latent TGF- $\beta_{1}$, whereas the GGWSHW sequence binds active TGF- $\beta_{1}$ and potentially orients the TSP molecule. $^{57}$ TSP-2 lacks the KRFK sequence in the TSR, but contains the GGWSHW motif, and therefore can bind, but not activate TGF- $\beta_{1}$. The significance of TSP-1-mediated TGF- $\beta_{1}$ activation is supported by studies in vivo. Histological abnormalities, e.g., leukocyte infiltration and inflammatory changes in the lung and pancreas, are strikingly similar in young TSP-1-null mice and TGF- $\beta_{1}$-null mice. ${ }^{58}$ Moreover, these abnormalities could be partially reversed by treatment of TSP-1-null mice with KRFK, a TSP-1-derived peptide that binds the KRFK motif and activates TGF- $\beta_{1}$. The synthetic peptide GGWSHW, based on a sequence in the TSR of TSP-1 and TSP-2, inhibited activation of TGF- $\beta_{1}$ by TSP- 1 . In addition, TSP- 2 inhibits activation of latent TGF- $\beta_{1}$ by TSP-1, presumably via competitive binding to TGF- $\beta_{1}$ when both TSPs are present. ${ }^{57}$ Whether this possibility is the case in remodeling tissues is questionable, since the expression of TSP-1 and TSP-2 is differentially controlled. ${ }^{59}$ Recently, the importance of TSP-1mediated TGF- $\beta_{1}$ activation during cardiac remodeling has been shown, as a peptide antagonist of TSP-1-dependent TGF- $\beta_{1}$ activation prevented fibrosis and improved cardiac function. ${ }^{60}$ Analyses of active TGF- $\beta_{1}$ levels in TSP-2-null mice during LVH would provide an insight in the role of TSP-1 and TSP-2 expression in controlling TGF- $\beta_{1}$ activation during $\mathrm{LVH}$ remodeling.

\section{Thrombospondin-integrin interaction in cardiac hypertrophy}

Integrins and CD47/IAP are cell surface receptors that are important in physiological cell functioning by mediating interactions between cells and their ECM. In addition to their structural role in the anchoring of cells, integrins participate in signal transduction from the "outside to inside" and "inside to outside" of the cell ${ }^{5}$. Therefore, integrin and CD47/IAP signaling plays a crucial role in controlling cell behavior in response to various stimuli, via regulation of processes such as cell adhesion, migration, proliferation, survival and apoptosis. ${ }^{5,61}$ In the heart, integrins are involved in normal cardiac mechanotransduction, and have been shown to be indispensible during cardiac remodeling. ${ }^{62}$ Focal adhesion kinase (FAK) and integrin-linked kinase (ILK) are downstream targets of integrin signaling and are implicated in cardiac remodeling. ${ }^{5}$ FAK-null mice developed dilated cardiomyopathy in response to pressure overload due to the lack of a proper hypertrophic response. ${ }^{63}$ Zebrafish with a mutation in the ILK-gene and ILK-null mice both display spontaneous cardiac failure ${ }^{64,65}$, and ILK expression has been associated with the development of cardiac hypertrophy. ${ }^{66}$

Together these studies emphasize that interactions with integrins and subsequent activation of its downstream targets are involved in cardiac failure. Several studies 
have identified TSPs as ligands for integrins and CD47/IAP. The RGD-containing type-3 repeat of TSP-1 was reported to interact with $\alpha v \beta 3$ integrin in human endothelial cells to mediate cell adhesion in vitro. ${ }^{67}$ Four $\beta 1$ integrin binding sites have been mapped to the N-terminus of TSP-1. The $\alpha 3 \beta 1$ integrin recognizes a NVR motif and stimulated angiogenesis and endothelial cell behavior. ${ }^{68,69}$ A second integrin, $\alpha 4 \beta 1$, was mapped to the LDVP sequence in TSP-1 that was also conserved in TSP-2. Interaction of TSP-1 with $\alpha 4 \beta 1$ on vascular endothelial cells regulates angiogenesis in vivo and affects cell proliferation, adhesion, and migration in vitro. ${ }^{70}$ Like $\alpha 4 \beta 1$, the binding site for $\alpha 6 \beta 1$ was conserved in both TSP- 1 and TSP- 2 and was reported to mediate adhesion and chemotaxis of microvascular endothelial cells. ${ }^{71}$ Recently, a fourth integrin, $\alpha 9 \beta 1$, was recognized as a receptor for TSP-1 that induces endothelial cell proliferation and migration and thereby modulates angiogenesis. ${ }^{67}$ In vascular smooth muscle cells (VSMC), TSP-1 can induce FAK phosphorylation, which is essential for migration. ${ }^{72,73}$ In addition, Gao et al. showed that TSP-1 also binds to CD47/IAP that in turn associates with $\alpha v \beta 3$ and leads to cell spreading, an activity that was correlated with increased FAK phosphorylation. ${ }^{74}$ These studies clearly demonstrate a role for TSPs in the modulation of integrin signaling, particularly in endothelial cells and VSMC during vascular remodeling. Although TSPs are recognized as binding partners of integrins and CD47/IAP and can activate FAK, no direct evidence for a role of TSPs in the stretchsensing machinery is available. Therefore, their importance in the integrin-mediated hypertrophic response remains to be elucidated.

\section{Thrombospondins and angiogenesis in cardiac hypertrophy}

TSP-1 and TSP-2 are recognized as potent inhibitors of angiogenesis [reviewed in ${ }^{75}$ and see review by Bornstein in this issue]. TSPs exert their anti-angiogenic effects through several mechanisms: inhibition of endothelial cell-cycle progression, prevention of endothelial cell mobilization, and/or induction of endothelial cell apoptosis ${ }^{33,76,77}$ (Figures 1 and 2). In addition, TSP-2 influences angiogenesis through modulation of MMP activity. ${ }^{18}$ Pathological hypertrophy is associated with a reduced capillary density, as found in several human cardiac diseases. ${ }^{78,79} \mathrm{NO}$ and Akt signaling are important in the process of angiogenesis and have been implicated in physiological and pathological cardiac function. $^{80}$

First recognized as an endothelial-derived relaxing factor ${ }^{81}$, NO is produced through conversion of L-arginine to L-citrulline by three NO synthases (nNOS, eNOS, and iNOS). ${ }^{82}$ In the heart, NO limits cardiac remodeling after $\mathrm{MI}$ and participates in the control of heart rate and contractility. ${ }^{80}$ In comparison with WT explants, TSP-1-null tissue explants show an enhanced angiogenic response elicited by $\mathrm{NO} .^{83} \mathrm{NO}$ induced endothelial cell proliferation, associated with a decrease in TSP-1 levels that was dependent on phosphorylation of ERK, whereas exogenous TSP-1 can suppress NO mediated ERK phosphorylation, data indicating a feedback loop between TSP-1 and NO. ${ }^{84}$ NO stimulates endothelial cell motility and adhesion to type I collagen in a cGMP- 
dependent manner. Picomolar concentrations of exogenous TSP-1 potently block NOmediated stimulation of CGMP. ${ }^{83}$ In addition, recombinant type-1 repeats of TSP-1 and a CD36 agonist antibody have a similar effect, whereas the N-terminal domain of TSP-1 did not inhibit NO-mediated stimulation of cGMP. These results indicate that the inhibitory effects of TSP-1 on pro-angiogenic NO signaling are mediated through binding to CD36. Later, it was reported that TSP-1 inhibits NO stimulated responses in vascular smooth muscle cells in an identical manner as previously described in endothelial cells. $^{85}$ A study in CD47/IAP-null cells demonstrated that, whereas ligation of CD36 is sufficient to block NO-mediated responses in VSMC and endothelial cells, only CD47/IAP is crucial for TSP-1 to exert its inhibitory effects. ${ }^{86}$ TSP-2 shares the Cterminal binding motif for CD47/IAP with TSP-1, and both TSPs have been found to regulate inflammatory responses through this receptor. ${ }^{87}$ However, TSP-2 binds less avidly to CD47/IAP-expressing cells compared to TSP-1. Consistent with these findings TSP-2 inhibits NO-mediated cGMP synthesis in vascular cells less efficiently. ${ }^{88}$

Short-term and long-term Akt activation have different effects on cardiac hypertrophy and angiogenesis. ${ }^{89}$ Short-term activation of Akt in transgenic mice results in physiological hypertrophy and concomitant enhanced angiogenesis, responses resulting in maintenance of vascular density. ${ }^{89}$ This capillary growth is mediated by increased expression of vascular endothelial growth factor (VEGF) and angiopoietin-2 (Ang-2) after short-term Akt activation, which is dependent on mTOR. ${ }^{89}$ Long-term activation of Akt, on the other hand, results in down regulation of VEGF and Ang-2, possibly due to decreased expression of $\mathrm{mTOR}$, and is accompanied by a decrease in capillary density. Akt-null mice displayed enhanced angiogenic responses and an ECM reduced in density, which may facilitate growth of new capillaries in the skin of Akt-null mice. ${ }^{90}$ Importantly, the observed abnormalities in collagen organization, enhanced angiogenesis, and vascular leakage, closely resemble the phenotype of mice lacking TSP-1 and TSP-2. ${ }^{90}$ TSP-1 and TSP-2 are decreased in the tissues of Akt-null mice, and the increased angiogenesis found in these animals could be normalized by re-expression of TSP-1 and TSP-2. ${ }^{90}$ Thus, the overall effect of Akt on angiogenesis is suppressive and involves controlling the expression of angiostatic TSP-1 and TSP-2. Recently, the link between TSPs and Akt in angiogenesis was confirmed by a study demonstrating that TSP-1 modulates microvascular remodeling in the retina by antagonizing Akt-signaling triggered by VEGF, whereas no significant effects of TSP-2 were reported. ${ }^{91,92}$ Whether or not inhibition of VEGF-induced Akt-signaling by TSP-1 depends on direct blockade of VEGF signaling, or sequestration of Akt-signaling, remains uncertain. Interestingly, recent evidence revealed increased basal VEGF levels in TSP-1-null mice, associated with increased capillary density in cardiac and skeletal muscle. These data indicate that TSP-1 regulates angiogenesis via a direct effect on VEGF. ${ }^{19}$ Whether the upregulation of TSPs during cardiac hypertrophy is important for the balance between myocyte and capillary growth, via modulation of NO- and/or Akt-signaling, remains to be investigated. 


\section{Thrombospondins in cardiac remodeling after acute myocardial infarction}

$\mathrm{MI}$ is one of the major risk factors in the development of heart failure, and accounts for $62 \%$ of all cases. ${ }^{93}$ During $\mathrm{MI}$, part of the heart is subjected to an abrupt lack of perfusion that leads to rapid death of the cardiac myocytes and the formation of a scar. Grossly, infarct healing consists of three overlapping phases; the inflammatory phase, the proliferative phase, and the maturation phase. ${ }^{94}$ The inflammatory phase takes place within hours after the onset of $\mathrm{Ml}$ and is marked by the influx of leukocytes that remove the dead myocytes, followed by the influx of other inflammatory cells. Two to 3 days after $\mathrm{Ml}$, the onset of the proliferative phase of infarct healing begins with the formation of granulation tissue, which is rich in inflammatory cells and myofibroblasts that are capable of producing large amounts of ECM proteins. In parallel with the proliferation phase of infarct healing, new blood vessels start to appear in the infarcted area. Finally, after 2-3 weeks, the cell-rich granulation tissue evolves into a mature, collagen-based scar, characterized by the disappearance of cells and by the cross-linking of secreted collagen. During all phases of infarct healing, the composition of the ECM plays a crucial role in regulating cell behavior by modulation of cell phenotype and gene expression. ${ }^{95}$ The following paragraphs discuss the expression and function of TSPs during the three phases of wound healing after MI. We discuss only TSP-1 and TSP-2 because they are known to play a role in infarct healing.

\section{Expression of TSPs after acute myocardial infarction}

The expression of TSP-1 precedes that of TSP-2 and partially overlaps it during the process of wound healing. This important feature could explain some of the phenotypes found in the respective null mice, as described below (Table 1). TSP-1 is secreted by inflammatory cells, and its expression is high during early phases of wound healing, whereas TSP-2 is secreted mostly by fibroblasts, and is therefore expressed at high levels during later stages of wound healing. ${ }^{59,96}$

\section{Thrombospondins and the inflammatory response}

A role of TSP-1 and TSP-2 in wound repair is suggested by their dynamic expression pattern during the different phases of wound healing. TSP-1 is secreted by inflammatory cells during the acute phase of tissue repair, whereas its expression drops during the proliferative phase of healing. ${ }^{96}$ The role of TSP-1 in the inflammatory phase after MI has been described by Frangogiannis et al., who discovered that TSP-1 prevents expansion of healing myocardial infarcts ${ }^{10}$ (Table 1). First, TSP-1 expression was enhanced in a canine ischemia-reperfusion model. Thereafter, they extended this model to TSP-1-null and WT mice. In infarcted WT mice TSP-1 protein was noted after 24 hours to 7 days, and expression was localized to the border zone of the infarct. ${ }^{10} \mathrm{TSP}$ 1-null mice displayed higher levels of chemokine and cytokine transcripts in healing 
infarcts, which were associated with increased and prolonged abundance of macrophages and myofibroblasts in the infarct zone, as well as in remote, non-infarcted myocardium. This observation indicates that TSP-1-null mice have an increased inflammatory response after ischemia-reperfusion, which correlates with the enhanced ventricular dilation after $\mathrm{Ml}$ in these animals. The mechanism responsible for mediating the inflammatory response by TSP-1 after MI is likely to involve the activation of local TGF- $\beta_{1}$. As previously discussed, TSP-1 is a major activator of TGF- $\beta_{1}$ in vivo. ${ }^{58}$ In non-infarcted murine hearts TSP-1 is not expressed, and levels of chemokine and cytokine transcripts are similar between WT and TSP-1-null animals. However, infarcted hearts show a strong inflammatory response with marked upregulation of TGF- $\beta_{1} \cdot{ }^{97}$ In addition, infarcted hearts of TSP-1- null mice showed a trend towards decreased phosphorylation of the intracellular effector of TGF- $\beta_{1}$, Smad2. Alternatively, the angiostatic effects of TSP-1 may regulate infarct healing. However, no differences in microvascular profiles between WT and TSP-1- null mice were reported after MI. ${ }^{10}$ Therefore, the selective expression of TSP-1 in the infarct border zone is suggested to function as a barrier that locally suppresses inflammatory chemokines and cytokines through its TGF- $\beta_{1}$-activating effects, and prevents extension of the inflammatory process into the non-infarcted area.

In contrast to TSP-1, the structurally similar TSP-2 does not activate latent TGF- $\beta_{1}$ and is therefore likely to affect the inflammatory process through other mechanisms. Different studies concerning the role of TSP-2 in the inflammatory response reported contradictory results. In oxazolone-induced inflammation of the skin, lack of TSP-2 results in an enhanced inflammatory response. TSP-2-null mice exhibited increased angiogenesis, edema, and inflammatory infiltration. ${ }^{98}$ Moreover, microvascular leakage was enhanced in the inflamed skin of TSP-2-null mice, a result indicating that TSP-2 controls vascular permeability during inflammation. Another study showed similar phenotypes in CD47/IAP-, TSP-1-, and TSP-2-null mice after oxazolone-induced inflammation. The authors suggested that the observed prolonged inflammation resulted from a deficiency in T-cell apoptosis in these mice that was associated with a defect in TSP-CD47/IAP interactions. ${ }^{87}$ In contrast to oxazolone-induced inflammation, the early phase of dermal skin wound healing (0-4 days) did not differ between WT and TSP-2-null mice, and there were no signs of increased inflammatory infiltration. However, 7 days after wounding, progressive and significant differences were revealed between WT and TSP-2-null mice, the latter showing accelerated healing with irregular granulation tissue, and increased angiogenesis. ${ }^{59,} 99$ Therefore, one might speculate that TSP-2 affects the inflammatory response at a later phase of wound healing, in comparison with TSP-1, presumably via regulation of vascular permeability and T-cell apoptosis during inflammation. With respect to the role of TSP-2 in MI, a high incidence of cardiac rupture in TSP-2-null mice within 3 days after acute MI was reported. ${ }^{100}$ The inflammatory response in the hearts of these TSP-2-null mice has not been evaluated. These findings imply an important role for TSP-2 in controlling the structural integrity during ECM remodeling in the heart. 


\section{Thrombospondins and the proliferative phase of infarct healing}

After the phase of acute inflammation, 2 to 3 days after $\mathrm{MI}$, granulation tissue begins to form in the border zone of the infarct. Key features of this granulation tissue are the abundance of inflammatory cells, myofibroblasts and small blood vessels. Both TSP-1 and TSP-2 are potent inhibitors of angiogenesis and exert their angiostatic effects via interaction with CD36 and/or CD47/IAP ${ }^{77,83}$ (Figures 1 and 2). TSP-1 mRNA is markedly induced 6-24 hours after reperfusion, and TSP-1 immunoreactivity showed specific localization in the infarct border zone 7 to 28 days after MI. In addition, the vascular network in TSP-1-null infarcts was comparable to neovascularization in WT mice. ${ }^{10}$ Consistent with these findings, angiogenesis has not been found to be increased in skin wounds of mice lacking TSP-1. ${ }^{96}$ A plausible explanation for this observation is that TSP-1 is not expressed during the late proliferative phase and during remodeling, when neovascularization occurs. TSP-1 is a major activator of latent TGF- $\beta_{1}$, an important factor in myofibroblast differentiation. Surprisingly, TSP-1-null infarcts contained higher numbers of myofibroblasts. ${ }^{10}$ These data indicate that TSP-1 is more involved in controlling the inflammatory response and preventing expansion of the granulation tissue into the non-infarcted area, rather than affecting angiogenesis, during this phase of wound healing.

The effects of TSP-2 on angiogenesis during the proliferative phase after MI cannot be studied directly, due to the early cardiac ruptures that occur in TSP-2-null animals after acute MI. ${ }^{100}$ However, during cutaneous wound healing TSP-2 exhibits a time/phase dependent expression pattern. In early wounds, when there is increased angiogenesis, TSP-2 is sparse and can be localized to a small number of fibroblasts. Levels of TSP-2 become high, and its expression widespread, during late stages of healing, which coincide with ECM remodeling and vascular regression. ${ }^{99}$ In contrast to TSP1-null mice, TSP-2-null mice exhibited increased vascular density after dermal wound healing together with the presence of an abnormal, loosely organized collagen matrix. $^{59,99}$ These findings are consistent with the phenotypes of TSP-1 and TSP-2-null mice that show alterations in the early and late phases of wound healing, respectively. TSP-2 is likely to be important for controlling angiogenesis and the integrity of the newly formed matrix during later phases of infarct healing (Figure 2).

\section{Thrombospondins and maturation of the infarct scar}

Maturation of the infarct scar is characterized by removal of inflammatory cells, regression of blood vessels, and cross-linking of the deposited collagen. ${ }^{101}$ Since the levels of TSP-1 are low during late stages of wound healing, the protein was expected to play a minor role during infarct maturation. After ischemia-reperfusion, TSP-1 was observed up to 28 days after injury; however, its expression was highly selective for the infarct border zone, and was minimal in the infarct center. These data support the hypothesis that TSP-1 is not critical for infarct scar maturation. ${ }^{10}$ However, TSP-1 could 
affect maturation of the infarct scar through the activities of TGF- $\beta_{1}$, as TGF- $\beta_{1}$ is known to promote fibrosis via enhanced collagen deposition ${ }^{102}$ (Figure 1). Furthermore, TGF- $\beta_{1}$ inhibits MMP activities and increases synthesis of proteinase inhibitors such as plasminogen activator inhibitor (PAI) and TIMPs. ${ }^{103}$ Consistent with these functions, enhanced MMP-9 activity was reported in the infarct border zone in the absence of TSP-1, which in turn could explain the enhanced ventricular dilation after MI in TSP1-null mice. Whether TSP-1 is of significant importance during this phase of late infarct healing remains to be determined.

In contrast to TSP-1, TSP-2 is expressed during the phase of scar maturation, and TSP-2-null mice show abnormalities in collagen fibril formation. TSP-2 might therefore be an important regulator of infarct scar formation. ${ }^{104}$ By scanning electron microscopy, abnormal loop-like fibers of large diameter were detected in the dermis of TSP-2null mice. In addition, transmission electron microscopy showed that collagen fibrils in the dermis of TSP-2-null mice were wider in diameter, in comparison to WT fibrils. ${ }^{104}$ The abnormally organized collagen matrix might be caused by increased levels of MMP-2 and MMP-9 in wounds of TSP-2-null mice. ${ }^{59}$ The relevance of modulation of MMP activities by TSP-2 was supported by the observation that peak levels of MMP-2 and MMP-9 in the wounds of TSP-2-null mice coincide with the highest content of TSP2 in WT wounds. Together these studies indicate that TSP-2 is a crucial regulator of the integrity of the cardiac ECM during maturation of the infarct scar, presumably via regulation of MMP activity, and subsequent deposition of a cross-linked collagen matrix (Figure 2).

\section{Thrombospondin polymorphisms and myocardial infarction}

The importance of TSPs in infarct healing has been established in the above-described mouse models of acute MI. However, it is important to know whether TSPs are also involved in human cardiac diseases. This paragraph describes their potential role in the progression of atherosclerosis, plaque rupture and platelet biology, thereby linking TSP gene alterations to the risk of $\mathrm{MI}$.

Topol et al. were the first to describe a link between single nucleotide polymorphisms (SNPS) in TSP genes and MI. ${ }^{105}$ The SNPs affected the amino acid sequences of TSP-1 (Asn700Ser, 2210A/G) and TSP-4 (Ala387Pro, 1186G/C), whereas for TSP-2 a SNP was found in the $3^{\prime}$ untranslated region (UTR) (3949T/G). The SNP in TSP-1 lowers its capacity for calcium binding, an alteration that could influence the structure and function of this matricellular protein. In platelets, the TSP-1 SNP is associated with increased cell-surface expression of TSP-1 and enhanced platelet aggregation. These results provide a mechanism for the increased risk of $\mathrm{MI}$ in individuals bearing the TSP1 SNP. The TSP-4 387Pro variant is dominant, and exhibits increased calcium binding, relative to WT TSP-4, although the expression and processing of TSP-4 are not affected in cells with this SNP. The TSP-4 SNP alters endothelial cell behavior by its interference with adhesion and proliferation. Blocking endothelial cell repair, increasing smooth 
muscle cell proliferation, and augmenting neutrophil activation by the TSP-4 387Pro variant could result in atherosclerotic lesions and an increased risk of MI. The SNP found in the 3' UTR of TSP-2 mRNA results in a different secondary structure, as predicted by the M-fold model, which might affect protein expression. Consistent with this possibility, the expression of a luciferase reporter with the TSP-2 3949G variant was substantially lower in human umbilical vein endothelial cells, in comparison to that of the TSP-2 3949T variant. These data suggest decreased expression of TSP-2 in the vascular wall of individuals with the 3949G SNP.

However, the association between TSP SNPS and MI is not a consistent finding. Koch et al. determined the genotypes related to the SNPS in the genes for TSP-1, TSP-2, and TSP-4 in a large, case-control sample of MI and performed a meta-analysis of data from their own and from previous studies. ${ }^{106}$ They described mildly, but significantly, elevated risks of $\mathrm{Ml}$ in individuals carrying the TSP-2 3949G SNP, whereas no correlation was found for the TSP-1 and TSP-4 SNP variant with MI. Their meta-analysis of available data revealed that none of the polymorphisms was linked with the risk of MI.

Because studies with TSP-null mice indicate a role for TSP-1 and TSP-2 in infarct healing, it would be tempting to investigate the influence of TSP SNPS on infarct healing and the transition toward heart failure. These studies would provide evidence confirming the importance of TSPs in cardiac remodeling after acute MI.

\section{Thrombospondins as key players during cardiac remodeling}

In the complex process of cardiac remodeling, many growth factors, cytokines, and other proteins have been recognized as key players. Lately, non-structural proteins residing in the ECM, such as certain matricellular proteins including periostin, were highlighted as important factors influencing cardiac remodeling. In this review, we have discussed the functions and possible actions of the matricellular TSPs during cardiac remodeling (Figures 1 and 2 ).

First, evidence supporting the notion that TSPs are involved in cardiac remodeling was provided by different studies that reported upregulation of different TSPs after cardiac injury. Several groups reported elevated expression of TSP-1, TSP-2, TSP-3 and TSP-4 in different models of pressure overload (Table 1). Schroen et al. identified TSP-2 as an important regulator of the transition from compensated LVH towards heart failure. $^{11}$ TSP-2-null mice, after Ang-Il infusion, showed advanced myocardial rupture accompanied by increased levels of MMP-2. MMPs and their endogenous inhibitors (TIMPS) are actively involved in hypertrophic cardiac remodeling. TSP-1 and TSP-2 contain TSR motifs that are able to interact with MMPs to regulate their activity. Therefore, TSPs are likely to play a role in the process of LVH by controlling the MMPTIMP balance and thereby regulating breakdown of the ECM. A second mechanism by which TSPs could affect LVH depends on the regulation of the activity of the profibrotic, anti-inflammatory factor, TGF- $\beta_{1}$, which is synthesized after induction of the 
RAS and contributes to myocardial remodeling. TSP-1 binds TGF- $\beta_{1}$ via its GGWSHW motif and can subsequently activate the protein through its KRFK motif.

The complex domain structure of TSP-1 and TSP-2 allows them to interact with a large number of cell surface receptors, including integrins and CD47/IAP, that participate in signal transduction. TSPs could affect the development of LVH as regulators of angiogenesis via interactions with integrins, CD36 and CD47/IAP or as targets of chronic Akt activation and inhibitors of NO signaling.

Studies that investigated TSPs after MI suggested that TSP-1 and TSP-2 have distinct functions depending on their spatiotemporal expression. Models of wound healing in mice revealed that TSP-1 is markedly induced during the acute inflammatory phase and drops during later phases of healing, whereas TSP-2 expression is limited to a small number of fibroblasts during inflammation and peaks in the proliferative phase and during scar maturation. In a setting of MI, TSP-1-null infarcts revealed more inflammation and expansion of the infarct, whereas no differences in vascularization were reported. These data indicate that TSP-1 functions to control the inflammatory response and prevent infarct expansion, most likely via regulation of TGF- $\beta_{1}$ activity during the first stage of infarct healing. That TSP-2-null mice display pronounced cardiac rupture after $\mathrm{MI}$, indicates a crucial role for TSP-2 in regulation of the integrity of the cardiac ECM during remodeling, presumably through regulation of the activities of MMP-2 and MMP-9.

We speculate that TSPs are crucial mediators of cardiac remodeling through their influence on various processes, due to their spatiotemporal expression pattern and structure. TSPs might directly influence cardiomyocyte function through interaction with various surface receptors such as integrins, CD47/IAP, and CD36, whereas they can regulate ECM remodeling through their effect on MMP activation. Also, their effects on inflammation and angiogenesis might be important during cardiac remodeling. Thus, we postulate that the TSPs are important in a variety of cardiac diseases, as TSPs can modulate cell behavior through interactions with different surface receptors, with their specific effects depending on the cell type and the nature of the remodeling process.

Studies addressing the role of TSPs in remodeling after cardiac injury are limited. However, in this review we present strong evidence that TSPs are key players during hypertrophic and ischemic cardiac remodeling. Increasing our understanding of these proteins affords new opportunities for intervention to prevent the transition toward heart failure in patients suffering from cardiac diseases.

\section{Acknowledgments}

This study was supported by research grants of the Netherlands Heart Foundation (NHS 2005B082, NHS 2007B036, 2008B011), and a VIDI grant of the Dutch Research Foudation to $\mathrm{SH}$. 


\section{References}

1 Braunwald E. Shattuck lecture--cardiovascular medicine at the turn of the millennium: triumphs, concerns, and opportunities. N Engl J Med. 1997;337(19):1360-1369.

2 Vasan RS, Benjamin EJ, Levy D. Prevalence, clinical features and prognosis of diastolic heart failure: an epidemiologic perspective. J Am Coll Cardiol. 1995;26(7):1565-1574.

3 Weir RA, McMurray JJ, Velazquez EJ. Epidemiology of heart failure and left ventricular systolic dysfunction after acute myocardial infarction: prevalence, clinical characteristics, and prognostic importance. Am J Cardiol. 2006;97(10A):13F-25F.

4 Rosenkranz S. TGF-beta1 and angiotensin networking in cardiac remodeling. Cardiovasc Res. 2004;63(3):423-432.

5 Lal H, Verma SK, Foster DM, Golden HB, Reneau JC, Watson LE, Singh H, Dostal DE. Integrins and proximal signaling mechanisms in cardiovascular disease. Front Biosci. 2009;14:2307-2334.

6 Manso AM, Elsherif L, Kang SM, Ross RS. Integrins, membrane-type matrix metalloproteinases and ADAMs: potential implications for cardiac remodeling. Cardiovasc Res. 2006;69(3):574-584.

7 Spinale FG. Myocardial matrix remodeling and the matrix metalloproteinases: influence on cardiac form and function. Physiol Rev. 2007;87(4):1285-1342.

8 Swynghedauw B. Molecular mechanisms of myocardial remodeling. Physiol Rev. 1999;79(1):215-262.

9 Schellings MW, Pinto YM, Heymans S. Matricellular proteins in the heart: possible role during stress and remodeling. Cardiovasc Res. 2004;64(1):24-31.

10 Frangogiannis NG, Ren G, Dewald O, Zymek P, Haudek S, Koerting A, Winkelmann K, Michael LH, Lawler J, Entman ML. Critical role of endogenous thrombospondin-1 in preventing expansion of healing myocardial infarcts. Circulation. 2005;111(22):2935-2942.

11 Schroen B, Heymans S, Sharma U, Blankesteijn WM, Pokharel S, Cleutjens JP, Porter JG, Evelo CT, Duisters R, van Leeuwen RE, Janssen BJ, Debets JJ, Smits JF, Daemen MJ, Crijns HJ, Bornstein P, Pinto YM. Thrombospondin-2 is essential for myocardial matrix integrity: increased expression identifies failureprone cardiac hypertrophy. Circ Res. 2004;95(5):515-522.

12 Collins AR, Schnee J, Wang W, Kim S, Fishbein MC, Bruemmer D, Law RE, Nicholas S, Ross RS, Hsueh WA. Osteopontin modulates angiotensin II-induced fibrosis in the intact murine heart. J Am Coll Cardiol. 2004;43(9):1698-1705.

13 Trueblood NA, Xie Z, Communal C, Sam F, Ngoy S, Liaw L, Jenkins AW, Wang J, Sawyer DB, Bing OH, Apstein CS, Colucci WS, Singh K. Exaggerated left ventricular dilation and reduced collagen deposition after myocardial infarction in mice lacking osteopontin. Circ Res. 2001;88(10):1080-1087.

14 Shimazaki M, Nakamura K, Kii I, Kashima T, Amizuka N, Li M, Saito M, Fukuda K, Nishiyama T, Kitajima S, Saga Y, Fukayama M, Sata M, Kudo A. Periostin is essential for cardiac healing after acute myocardial infarction. J Exp Med. 2008;205(2):295-303.

15 Schellings MW, Vanhoutte D, Swinnen M, Cleutjens JP, Debets J, van Leeuwen RE, d'Hooge J, Van de Werf F, Carmeliet P, Pinto YM, Sage EH, Heymans S. Absence of SPARC results in increased cardiac rupture and dysfunction after acute myocardial infarction. J Exp Med. 2009;206(1):113-123.

16 Chatila K, Ren G, Xia Y, Huebener P, Bujak M, Frangogiannis NG. The role of the thrombospondins in healing myocardial infarcts. Cardiovasc Hematol Agents Med Chem. 2007;5(1):21-27.

17 Isenberg JS, Qin Y, Maxhimer JB, Sipes JM, Despres D, Schnermann J, Frazier WA, Roberts DD. Thrombospondin-1 and CD47 regulate blood pressure and cardiac responses to vasoactive stress. Matrix Biol. 2009;28(2):110-119.

18 Krady MM, Zeng J, Yu J, MacLauchlan S, Skokos EA, Tian W, Bornstein P, Sessa WC, Kyriakides TR. Thrombospondin-2 modulates extracellular matrix remodeling during physiological angiogenesis. Am J Pathol. 2008;173(3):879-891.

19 Malek MH, Olfert Ph DI. Global Deletion of Thrombospondin-1 Increases Cardiac and Skeletal Muscle Capillarity and Exercise Capacity. Exp Physiol. 2009.

20 Gupta S, Das B, Sen S. Cardiac hypertrophy: mechanisms and therapeutic opportunities. Antioxid Redox Signal. 2007;9(6):623-652. 
21 Chaulet H, Lin F, Guo J, Owens WA, Michalicek J, Kesteven SH, Guan Z, Prall OW, Mearns BM, Feneley MP, Steinberg SF, Graham RM. Sustained augmentation of cardiac alpha1A-adrenergic drive results in pathological remodeling with contractile dysfunction, progressive fibrosis and reactivation of matricellular protein genes. J Mol Cell Cardiol. 2006;40(4):540-552.

22 Wang D, Oparil S, Feng JA, Li P, Perry G, Chen LB, Dai M, John SW, Chen YF. Effects of pressure overload on extracellular matrix expression in the heart of the atrial natriuretic peptide-null mouse. Hypertension. 2003;42(1):88-95.

23 Rysa J, Leskinen $\mathrm{H}$, Ilves $\mathrm{M}$, Ruskoaho $\mathrm{H}$. Distinct upregulation of extracellular matrix genes in transition from hypertrophy to hypertensive heart failure. Hypertension. 2005;45(5):927-933.

24 Mustonen E, Aro J, Puhakka J, Ilves M, Soini Y, Leskinen H, Ruskoaho H, Rysa J. Thrombospondin-4 expression is rapidly upregulated by cardiac overload. Biochem Biophys Res Commun. 2008;373(2):186191.

25 Tan FL, Moravec CS, Li J, Apperson-Hansen C, McCarthy PM, Young JB, Bond M. The gene expression fingerprint of human heart failure. Proc Natl Acad Sci U S A. 2002;99(17):11387-11392.

26 Yang Z, Kyriakides TR, Bornstein P. Matricellular proteins as modulators of cell-matrix interactions: adhesive defect in thrombospondin 2-null fibroblasts is a consequence of increased levels of matrix metalloproteinase-2. Mol Biol Cell. 2000;11(10):3353-3364.

27 Emonard H, Bellon G, Troeberg L, Berton A, Robinet A, Henriet P, Marbaix E, Kirkegaard K, Patthy L, Eeckhout $Y$, Nagase H, Hornebeck W, Courtoy PJ. Low density lipoprotein receptor-related protein mediates endocytic clearance of pro-MMP-2.TIMP-2 complex through a thrombospondin-independent mechanism. J Biol Chem. 2004;279(52):54944-54951.

28 Agah A, Kyriakides TR, Bornstein P. Proteolysis of cell-surface tissue transglutaminase by matrix metalloproteinase- 2 contributes to the adhesive defect and matrix abnormalities in thrombospondin-2-null fibroblasts and mice. Am J Pathol. 2005;167(1):81-88.

29 Yang Z, Strickland DK, Bornstein P. Extracellular matrix metalloproteinase 2 levels are regulated by the low density lipoprotein-related scavenger receptor and thrombospondin 2. J Biol Chem. 2001;276(11):8403-8408.

30 Maclauchlan S, Skokos EA, Agah A, Zeng J, Tian W, Davidson JM, Bornstein P, Kyriakides TR. Enhanced angiogenesis and reduced contraction in thrombospondin-2-null wounds is associated with increased levels of matrix metalloproteinases-2 and -9, and soluble VEGF. J Histochem Cytochem. 2009;57(4):301313.

31 Albo D, Shinohara T, Tuszynski GP. Up-regulation of matrix metalloproteinase 9 by thrombospondin 1 in gastric cancer. J Surg Res. 2002;108(1):51-60.

32 Qian X, Wang TN, Rothman VL, Nicosia RF, Tuszynski GP. Thrombospondin-1 modulates angiogenesis in vitro by up-regulation of matrix metalloproteinase- 9 in endothelial cells. Exp Cell Res. 1997;235(2):403412.

33 Rodriguez-Manzaneque JC, Lane TF, Ortega MA, Hynes RO, Lawler J, Iruela-Arispe ML. Thrombospondin1 suppresses spontaneous tumor growth and inhibits activation of matrix metalloproteinase-9 and mobilization of vascular endothelial growth factor. Proc Natl Acad Sci U S A. 2001;98(22):12485-12490.

34 Lee T, Esemuede N, Sumpio BE, Gahtan V. Thrombospondin-1 induces matrix metalloproteinase-2 activation in vascular smooth muscle cells. J Vasc Surg. 2003;38(1):147-154.

35 Taraboletti G, Morbidelli L, Donnini S, Parenti A, Granger HJ, Giavazzi R, Ziche M. The heparin binding 25 kDa fragment of thrombospondin-1 promotes angiogenesis and modulates gelatinase and TIMP-2 production in endothelial cells. FASEB J. 2000;14(12):1674-1676.

36 Donnini S, Morbidelli L, Taraboletti G, Ziche M. ERK1-2 and p38 MAPK regulate MMP/TIMP balance and function in response to thrombospondin-1 fragments in the microvascular endothelium. Life Sci. 2004;74(24):2975-2985.

37 Heineke J, Molkentin JD. Regulation of cardiac hypertrophy by intracellular signalling pathways. Nat Rev Mol Cell Biol. 2006;7(8):589-600.

38 Crabos M, Roth M, Hahn AW, Erne P. Characterization of angiotensin II receptors in cultured adult rat cardiac fibroblasts. Coupling to signaling systems and gene expression. J Clin Invest. 1994;93(6):23722378. 
39 Miyata S, Haneda T. Hypertrophic growth of cultured neonatal rat heart cells mediated by type 1 angiotensin II receptor. Am J Physiol. 1994;266(6 Pt 2):H2443-2451.

40 Schorb W, Booz GW, Dostal DE, Conrad KM, Chang KC, Baker KM. Angiotensin II is mitogenic in neonatal rat cardiac fibroblasts. Circ Res. 1993;72(6):1245-1254.

41 Villarreal FJ, Kim NN, Ungab GD, Printz MP, Dillmann WH. Identification of functional angiotensin II receptors on rat cardiac fibroblasts. Circulation. 1993;88(6):2849-2861.

42 Eghbali M, Tomek R, Sukhatme VP, Woods C, Bhambi B. Differential effects of transforming growth factor-beta 1 and phorbol myristate acetate on cardiac fibroblasts. Regulation of fibrillar collagen mRNAs and expression of early transcription factors. Circ Res. 1991;69(2):483-490.

43 Heimer R, Bashey RI, Kyle J, Jimenez SA. TGF-beta modulates the synthesis of proteoglycans by myocardial fibroblasts in culture. J Mol Cell Cardiol. 1995;27(10):2191-2198.

44 Sappino AP, Schurch W, Gabbiani G. Differentiation repertoire of fibroblastic cells: expression of cytoskeletal proteins as marker of phenotypic modulations. Lab Invest. 1990;63(2):144-161.

45 Tomasek JJ, Gabbiani G, Hinz B, Chaponnier C, Brown RA. Myofibroblasts and mechano-regulation of connective tissue remodelling. Nat Rev Mol Cell Biol. 2002;3(5):349-363.

46 Villarreal FJ, Lee AA, Dillmann WH, Giordano FJ. Adenovirus-mediated overexpression of human transforming growth factor-beta 1 in rat cardiac fibroblasts, myocytes and smooth muscle cells. J Mol Cell Cardiol. 1996;28(4):735-742.

47 Gray MO, Long CS, Kalinyak JE, Li HT, Karliner JS. Angiotensin II stimulates cardiac myocyte hypertrophy via paracrine release of TGF-beta 1 and endothelin-1 from fibroblasts. Cardiovasc Res. 1998;40(2):352363.

48 Tokuda K, Kai H, Kuwahara F, Yasukawa H, Tahara N, Kudo H, Takemiya K, Koga M, Yamamoto T, Imaizumi T. Pressure-independent effects of angiotensin II on hypertensive myocardial fibrosis. Hypertension. 2004;43(2):499-503.

49 Tomita H, Egashira K, Ohara Y, Takemoto M, Koyanagi M, Katoh M, Yamamoto H, Tamaki K, Shimokawa $\mathrm{H}$, Takeshita A. Early induction of transforming growth factor-beta via angiotensin II type 1 receptors contributes to cardiac fibrosis induced by long-term blockade of nitric oxide synthesis in rats. Hypertension. 1998;32(2):273-279.

50 Casscells W, Bazoberry F, Speir E, Thompson N, Flanders K, Kondaiah P, Ferrans VJ, Epstein SE, Sporn M. Transforming growth factor-beta 1 in normal heart and in myocardial infarction. Ann N Y Acad Sci. 1990;593:148-160.

51 Hao J, Ju H, Zhao S, Junaid A, Scammell-La Fleur T, Dixon IM. Elevation of expression of Smads 2, 3, and 4 , decorin and TGF-beta in the chronic phase of myocardial infarct scar healing. J Mol Cell Cardiol. 1999;31(3):667-678.

52 Takahashi N, Calderone A, Izzo NJ, Jr., Maki TM, Marsh JD, Colucci WS. Hypertrophic stimuli induce transforming growth factor-beta 1 expression in rat ventricular myocytes. J Clin Invest. 1994;94(4):1470-1476.

53 Thompson NL, Bazoberry F, Speir EH, Casscells W, Ferrans VJ, Flanders KC, Kondaiah P, Geiser AG, Sporn MB. Transforming growth factor beta-1 in acute myocardial infarction in rats. Growth Factors. 1988;1(1):91-99.

54 Villarreal FJ, Dillmann WH. Cardiac hypertrophy-induced changes in mRNA levels for TGF-beta 1, fibronectin, and collagen. Am J Physiol. 1992;262(6 Pt 2):H1861-1866.

55 Wunsch M, Sharma HS, Markert T, Bernotat-Danielowski S, Schott RJ, Kremer P, Bleese N, Schaper W. In situ localization of transforming growth factor beta 1 in porcine heart: enhanced expression after chronic coronary artery constriction. J Mol Cell Cardiol. 1991;23(9):1051-1062.

56 Hein S, Arnon E, Kostin S, Schonburg M, Elsasser A, Polyakova V, Bauer EP, Klovekorn WP, Schaper J. Progression from compensated hypertrophy to failure in the pressure-overloaded human heart: structural deterioration and compensatory mechanisms. Circulation. 2003;107(7):984-991.

57 Schultz-Cherry S, Chen H, Mosher DF, Misenheimer TM, Krutzsch HC, Roberts DD, Murphy-Ullrich JE. Regulation of transforming growth factor-beta activation by discrete sequences of thrombospondin $1 . \mathrm{J}$ Biol Chem. 1995;270(13):7304-7310. 
58 Crawford SE, Stellmach V, Murphy-Ullrich JE, Ribeiro SM, Lawler J, Hynes RO, Boivin GP, Bouck N. Thrombospondin-1 is a major activator of TGF-beta1 in vivo. Cell. 1998;93(7):1159-1170.

59 Bornstein P, Agah A, Kyriakides TR. The role of thrombospondins 1 and 2 in the regulation of cell-matrix interactions, collagen fibril formation, and the response to injury. Int J Biochem Cell Biol. 2004;36(6):1115-1125.

60 Belmadani S, Bernal J, Wei CC, Pallero MA, Dell'italia L, Murphy-Ullrich JE, Berecek KH. A thrombospondin-1 antagonist of transforming growth factor-beta activation blocks cardiomyopathy in rats with diabetes and elevated angiotensin II. Am J Pathol. 2007;171(3):777-789.

61 Isenberg JS, Roberts DD, Frazier WA. CD47: a new target in cardiovascular therapy. Arterioscler Thromb Vasc Biol. 2008;28(4):615-621.

62 Shai SY, Harpf AE, Babbitt CJ, Jordan MC, Fishbein MC, Chen J, Omura M, Leil TA, Becker KD, Jiang M, Smith DJ, Cherry SR, Loftus JC, Ross RS. Cardiac myocyte-specific excision of the beta1 integrin gene results in myocardial fibrosis and cardiac failure. Circ Res. 2002;90(4):458-464.

63 DiMichele LA, Doherty JT, Rojas M, Beggs HE, Reichardt LF, Mack CP, Taylor JM. Myocyte-restricted focal adhesion kinase deletion attenuates pressure overload-induced hypertrophy. Circ Res. 2006;99(6):636645.

64 Bendig G, Grimmler M, Huttner IG, Wessels G, Dahme T, Just S, Trano N, Katus HA, Fishman MC, Rottbauer W. Integrin-linked kinase, a novel component of the cardiac mechanical stretch sensor, controls contractility in the zebrafish heart. Genes Dev. 2006;20(17):2361-2372.

65 White DE, Coutu P, Shi YF, Tardif JC, Nattel S, St Arnaud R, Dedhar S, Muller WJ. Targeted ablation of ILK from the murine heart results in dilated cardiomyopathy and spontaneous heart failure. Genes Dev. 2006;20(17):2355-2360.

66 Lu H, Fedak PW, Dai X, Du C, Zhou YQ, Henkelman M, Mongroo PS, Lau A, Yamabi H, Hinek A, Husain M, Hannigan G, Coles JG. Integrin-linked kinase expression is elevated in human cardiac hypertrophy and induces hypertrophy in transgenic mice. Circulation. 2006;114(21):2271-2279.

67 Staniszewska I, Zaveri S, Del Valle L, Oliva I, Rothman VL, Croul SE, Roberts DD, Mosher DF, Tuszynski GP, Marcinkiewicz C. Interaction of alpha9beta1 integrin with thrombospondin-1 promotes angiogenesis. Circ Res. 2007;100(9):1308-1316.

68 Krutzsch HC, Choe BJ, Sipes JM, Guo N, Roberts DD. Identification of an alpha(3)beta(1) integrin recognition sequence in thrombospondin-1. J Biol Chem. 1999;274(34):24080-24086.

69 Chandrasekaran L, He CZ, Al-Barazi H, Krutzsch HC, Iruela-Arispe ML, Roberts DD. Cell contactdependent activation of alpha3beta1 integrin modulates endothelial cell responses to thrombospondin1. Mol Biol Cell. 2000;11(9):2885-2900.

70 Calzada MJ, Zhou L, Sipes JM, Zhang J, Krutzsch HC, Iruela-Arispe ML, Annis DS, Mosher DF, Roberts DD. Alpha4beta1 integrin mediates selective endothelial cell responses to thrombospondins 1 and 2 in vitro and modulates angiogenesis in vivo. Circ Res. 2004;94(4):462-470.

71 Calzada MJ, Sipes JM, Krutzsch HC, Yurchenco PD, Annis DS, Mosher DF, Roberts DD. Recognition of the $\mathrm{N}$-terminal modules of thrombospondin-1 and thrombospondin-2 by alpha6beta1 integrin. J Biol Chem. 2003;278(42):40679-40687.

72 Gahtan V, Wang XJ, Ikeda M, Willis Al, Tuszynski GP, Sumpio BE. Thrombospondin-1 induces activation of focal adhesion kinase in vascular smooth muscle cells. J Vasc Surg. 1999;29(6):1031-1036.

73 Wang XJ, Maier K, Fuse S, Willis Al, Olson E, Nesselroth S, Sumpio BE, Gahtan V. Thrombospondin-1induced migration is functionally dependent upon focal adhesion kinase. Vasc Endovascular Surg. 2008;42(3):256-262.

74 Gao AG, Lindberg FP, Dimitry JM, Brown EJ, Frazier WA. Thrombospondin modulates alpha v beta 3 function through integrin-associated protein. J Cell Biol. 1996;135(2):533-544.

75 Armstrong LC, Bornstein P. Thrombospondins 1 and 2 function as inhibitors of angiogenesis. Matrix Biol. 2003;22(1):63-71.

76 Armstrong LC, Bjorkblom B, Hankenson KD, Siadak AW, Stiles CE, Bornstein P. Thrombospondin 2 inhibits microvascular endothelial cell proliferation by a caspase-independent mechanism. Mol Biol Cell. 2002;13(6):1893-1905.

77 Simantov R, Silverstein RL. CD36: a critical anti-angiogenic receptor. Front Biosci. 2003;8:s874-882. 
78 Hudlicka O, Brown M, Egginton S. Angiogenesis in skeletal and cardiac muscle. Physiol Rev. 1992;72(2):369-417.

79 Karch R, Neumann F, Ullrich R, Neumuller J, Podesser BK, Neumann M, Schreiner W. The spatial pattern of coronary capillaries in patients with dilated, ischemic, or inflammatory cardiomyopathy. Cardiovasc Pathol. 2005;14(3):135-144.

80 Rastaldo R, Pagliaro P, Cappello S, Penna C, Mancardi D, Westerhof N, Losano G. Nitric oxide and cardiac function. Life Sci. 2007;81(10):779-793.

81 Ignarro LJ, Buga GM, Wood KS, Byrns RE, Chaudhuri G. Endothelium-derived relaxing factor produced and released from artery and vein is nitric oxide. Proc Natl Acad Sci U S A. 1987;84(24):9265-9269.

82 Isenberg JS, Frazier WA, Roberts DD. Thrombospondin-1: a physiological regulator of nitric oxide signaling. Cell Mol Life Sci. 2008.

83 Isenberg JS, Ridnour LA, Perruccio EM, Espey MG, Wink DA, Roberts DD. Thrombospondin-1 inhibits endothelial cell responses to nitric oxide in a cGMP-dependent manner. Proc Natl Acad Sci U S A. 2005;102(37):13141-13146.

84 Ridnour LA, Isenberg JS, Espey MG, Thomas DD, Roberts DD, Wink DA. Nitric oxide regulates angiogenesis through a functional switch involving thrombospondin-1. Proc Natl Acad Sci $U$ S A. 2005;102(37):13147-13152.

85 Isenberg JS, Wink DA, Roberts DD. Thrombospondin-1 antagonizes nitric oxide-stimulated vascular smooth muscle cell responses. Cardiovasc Res. 2006;71(4):785-793.

86 Isenberg JS, Ridnour LA, Dimitry J, Frazier WA, Wink DA, Roberts DD. CD47 is necessary for inhibition of nitric oxide-stimulated vascular cell responses by thrombospondin-1. J Biol Chem. 2006;281(36):2606926080.

87 Lamy L, Foussat A, Brown EJ, Bornstein P, Ticchioni M, Bernard A. Interactions between CD47 and thrombospondin reduce inflammation. J Immunol. 2007;178(9):5930-5939.

88 Isenberg JS, Annis DS, Pendrak ML, Ptaszynska M, Frazier WA, Mosher DF, Roberts DD. Differential interactions of thrombospondin-1, -2 , and -4 with CD47 and effects on CGMP signaling and ischemic injury responses. J Biol Chem. 2009;284(2):1116-1125.

89 Shiojima I, Sato K, Izumiya Y, Schiekofer S, Ito M, Liao R, Colucci WS, Walsh K. Disruption of coordinated cardiac hypertrophy and angiogenesis contributes to the transition to heart failure. J Clin Invest. 2005;115(8):2108-2118.

90 Chen J, Somanath PR, Razorenova O, Chen WS, Hay N, Bornstein P, Byzova TV. Akt1 regulates pathological angiogenesis, vascular maturation and permeability in vivo. Nat Med. 2005;11(11):1188-1196.

91 Sun J, Hopkins BD, Tsujikawa K, Perruzzi C, Adini I, Swerlick RA, Bornstein P, Lawler J, Benjamin LE. Thrombospondin-1 Modulates Vegf-a Mediated Akt Signaling and Capillary Survival in the Developing Retina. Am J Physiol Heart Circ Physiol. 2009.

92 Simantov R, Febbraio M, Silverstein RL. The antiangiogenic effect of thrombospondin-2 is mediated by CD36 and modulated by histidine-rich glycoprotein. Matrix Biol. 2005;24(1):27-34.

93 Bansal D, Chahoud G, Smith ES, Mehta JL. Prevention of heart failure. Curr Opin Cardiol. 2006;21(5):510-516.

94 Frangogiannis NG. The mechanistic basis of infarct healing. Antioxid Redox Signal. 2006;8(11-12):19071939.

95 Dobaczewski M, Bujak M, Zymek P, Ren G, Entman ML, Frangogiannis NG. Extracellular matrix remodeling in canine and mouse myocardial infarcts. Cell Tissue Res. 2006;324(3):475-488.

96 Agah A, Kyriakides TR, Lawler J, Bornstein P. The lack of thrombospondin-1 (TSP1) dictates the course of wound healing in double-TSP1/TSP2-null mice. Am J Pathol. 2002;161(3):831-839.

97 Dewald O, Ren G, Duerr GD, Zoerlein M, Klemm C, Gersch C, Tincey S, Michael LH, Entman ML, Frangogiannis NG. Of mice and dogs: species-specific differences in the inflammatory response following myocardial infarction. Am J Pathol. 2004;164(2):665-677.

98 Lange-Asschenfeldt B, Weninger W, Velasco P, Kyriakides TR, von Andrian UH, Bornstein P, Detmar M. Increased and prolonged inflammation and angiogenesis in delayed-type hypersensitivity reactions elicited in the skin of thrombospondin-2--deficient mice. Blood. 2002;99(2):538-545. 
99 Kyriakides TR, Tam JW, Bornstein P. Accelerated wound healing in mice with a disruption of the thrombospondin 2 gene. J Invest Dermatol. 1999;113(5):782-787.

100 Cleutjens J, Huynen F, Smits J, Bornstein P, Daemen M. Thrombospondin-2 deficiency in mice results in cardiac rupture early after myocardial infarction. Circ Res. 1999;100 (Suppl.):156.

101 Blankesteijn WM, Creemers E, Lutgens E, Cleutjens JP, Daemen MJ, Smits JF. Dynamics of cardiac wound healing following myocardial infarction: observations in genetically altered mice. Acta Physiol Scand. 2001;173(1):75-82.

102 Lijnen PJ, Petrov VV, Fagard RH. Induction of cardiac fibrosis by transforming growth factor-beta(1). Mol Genet Metab. 2000;71(1-2):418-435.

103 Schiller M, Javelaud D, Mauviel A. TGF-beta-induced SMAD signaling and gene regulation: consequences for extracellular matrix remodeling and wound healing. J Dermatol Sci. 2004;35(2):83-92.

104 Kyriakides TR, Zhu YH, Smith LT, Bain SD, Yang Z, Lin MT, Danielson KG, lozzo RV, LaMarca M, McKinney $\mathrm{CE}$, Ginns El, Bornstein P. Mice that lack thrombospondin 2 display connective tissue abnormalities that are associated with disordered collagen fibrillogenesis, an increased vascular density, and a bleeding diathesis. J Cell Biol. 1998;140(2):419-430.

105 Topol EJ, McCarthy J, Gabriel S, Moliterno DJ, Rogers WJ, Newby LK, Freedman M, Metivier J, Cannata R, O’Donnell CJ, Kottke-Marchant K, Murugesan G, Plow EF, Stenina O, Daley GQ. Single nucleotide polymorphisms in multiple novel thrombospondin genes may be associated with familial premature myocardial infarction. Circulation. 2001;104(22):2641-2644.

106 Koch W, Hoppmann P, Waha AD, Schomig A, Kastrati A. Polymorphisms in thrombospondin genes and myocardial infarction: a case-control study and a meta-analysis of available evidence. Hum Mol Genet. 2008. 


\section{Chapter 3}

\section{The absence of thrombospondin- 2 causes age- related dilated cardiomyopathy}

Melissa Swinnen*, Davy Vanhoutte*, Geert C. van Almen, Nazha Hamdani, Mark W.M. Schellings, Jan D'hooge, Jolanda van der Velden, Matthew S. Weaver, E. Helene Sage, Paul Bornstein, Fons K. Verheyen, Thierry VandenDriessche, Marinee K. Chuah, Dirk Westermann, Walter J. Paulus, Frans van de Werf, Blanche Schroen, Peter Carmeliet, Yigal M. Pinto, Stephane Heymans

*These authors contributed equally to this manuscript

Modified from Circulation. 2009 Oct;120(16):1585-15 


\begin{abstract}
Background: The progressive shift from a young to an aged heart is characterized by alterations in the cardiac matrix. The present study investigated whether the matricellular protein TSP-2 may affect cardiac dimensions and function with physiological aging of the heart.
\end{abstract}

Methods and Results: TSP-2 knockout (KO) and wild type (WT) mice were followed up to an age of 60 weeks. Survival rate, cardiac function and morphology did not differ at a young age in TSP-2 KO compared to WT mice. However, over $55 \%$ of the TSP-2 KO mice died between 24 and 60 weeks of age, whereas less then $10 \%$ of the WT mice succumbed. In the absence of TSP-2, older mice displayed a severe dilated cardiomyopathy with impaired systolic function, increased cardiac dilatation and fibrosis. Ultrastructural analysis revealed progressive myocyte stress and death, accompanied by an inflammatory response and replacement fibrosis in aging TSP-2 KO animals, whereas capillary or coronary morphology or density was not affected. Importantly, adeno-associated viral vector (AAV9)-mediated transfer of TSP-2 in 7 weeks old TSP-2 KO mice normalized their survival and prevented the dilated cardiomyopathy. In TSP-2 $\mathrm{KO}$ animals, age-related cardiomyopathy was accompanied by increased MMP-2- and decreased tissue-transglutaminase-2 activity, together with impaired collagen crosslinking. At the cardiomyocyte level, TSP-2 deficiency in vivo and its knockdown in vitro decreased the activation of the Akt survival pathway in cardiomyocytes.

Conclusions: TSP-2 expression in the heart protects against age-dependent dilated cardiomyopathy. 


\section{Introduction}

Whether aging of the heart and related functional changes should be regarded as a physiological or pathological process is a puzzling question. In the absence of other diseases such as hypertension, diabetes or detrimental environmental factors such as smoking, the heart is perfectly able to "survive" for a human lifespan. Hence, disease processes such as lamin A/C mutations that result in accelerated aging of the heart and dilated cardiomyopathy, point towards essential protective mechanisms that are mandatory for normal physiological aging of the heart. ${ }^{1,2}$

The extracellular matrix (ECM) is a crucial system that hinders functional and structural deterioration of the heart with aging. Matrix elements not only provide structural support, as collagen does, but are also implicated in maintaining cellular homeostasis and regulating intracellular signaling during normal cardiac physiology. ${ }^{2}$ Thrombospondin-2 (TSP-2) belongs to a family of non-structural matricellular proteins implicated in regulating cell-matrix interactions. Its expression is low in the normal postnatal heart, but reappears at high levels during cardiac pathology ${ }^{3,4}$, and helps to preserve cardiac integrity during hypertension. ${ }^{5}$ However, its function in normal physiological aging of the heart remains unknown. We therefore investigated whether TSP-2, by affecting survival pathways in cardiomyocytes, may provide necessary molecular support for the heart with aging, and thus increase lifespan. We provide data showing that hearts lacking TSP-2 progress towards dilated cardiomyopathy with advanced age, but have normal morphology and function at a young age. Importantly, post-natal adeno-asociated viral vector (AAV9)-mediated transfer of TSP-2 in young TSP-2 KO completely normalized their survival and prevented the development of cardiac failure. With aging, lack of TSP-2 resulted in decreased activation of the Src/Akt survival pathway, increased matrix metalloproteinase 2 (MMP-2) activity and decreased collagen cross-linking, leading to progressive cardiomyocyte dropout, and overall cardiac failure and dilatation.

\section{Methods}

An expanded methods section is provided in the Supplemental material (pag. 61).

\section{Transgenic mice and experimental procedures}

The study was approved by the Institutional Animal Research Committees and all experiments were performed according to official rules formulated in Dutch and Belgian law on care and use of experimental animals. Eight to sixty weeks-old TSP-2 knockout (KO) mice and their WT littermates on a C57BI6/129SvJ/EMS+Ter genetic background were used. ${ }^{6}$ At a young age, male and female mice were divided into three age groups for further analysis: young mice, 8 to 12 weeks old ( $n=20$ for TSP-2 WT mice and $n=20$ 
for TSP-2 KO mice); intermediate-age mice, 25 to 30 weeks old age ( $n=20$ for TSP-2 WT mice and $n=20$ for TSP-2 KO mice); and older mice, 50-60 weeks old ( $n=100$ for TSP-2 WT mice and $n=100$ for TSP-2 KO mice). Consequently, only mortality rates within the third group were included in the survival curve.

Next, to rescue cardiac TSP-2 expression, AAV-9 gene transfer of TSP-2 was performed in TSP-2 KO mice. Additional details on the construction and production of the AAV9-TSP-2 and AAV9-green fluorescent protein (GFP) vectors, with both TSP-2 and GFP driven under the cytomegalovirus (CMV) promoter, are provided in the data supplement at the end of this chapter. We injected $100 \mu \mathrm{L}$ containing $1 \times 10^{11}$ viral genomic copies of AAV9-TSP-2 or the control AAV9-GFP intra-venously into the tail vein of adult (7-week-old) male ( $n=10$ for AAV9-TSP-2, $n=25$ for AAV9-GFP) and female ( $n=19$ for AAV9-TSP-2, n=10 for AAV9-GFP) TSP-2 KO mice and monitored them until 60 weeks of age. All analyses were performed following standard operating procedures and confirmed by independent observers blinded to genotype or treatment group.

\section{Cardiac function, histopathological and molecular analysis}

After the study period, all mice were anaesthetized, followed by transthoracic echocardiographic examination. Subsequently, hearts were taken out and prepared for further histological and molecular analysis, including immunohistochemical and electron microscopic analysis, determination of zymographic MMP and tissue transglutaminase (tTG) activity with aging, RNA isolation and real time polymerase chain reaction, immunoblotting, integrin-linked kinase (ILK) activity and active transforming growth factor- $\beta$ (TGF $\beta$ ) assays, in vitro experimental approaches, force measurements in single permeabilized cardiomyocytes, and phosphorylation status of myofilament proteins. A detailed description of all experimental materials and methods is provided in the data supplement at the end of this chapter.

\section{Statistical analysis}

All data are expressed as mean \pm standard error of mean (SEM). Mann-Whitney $U$ test, unpaired $t$ test or 2-way ANOVA was used as appropriate to assess statistical significance between groups. Survival curves were obtained by the Kaplan-Meier method and compared by the log-rank test. A 2-sided value of $P<0.05$ was considered statistically significant. 


\section{Results}

\section{TSP-2 KO mice develop accelerated aging-induced cardiomyopathy}

To investigate the effect of TSP-2 on aging, a prospective study for mortality was conducted (Figure 1A). Whereas no significant mortality was noted until 20 weeks of age, the survival rate of TSP-2 KO mice progressively declined thereafter as compared to WT mice (Figure 1A). By 60 weeks of age $55 \%$ of the TSP-2 KO mice died (55 out of 100), whereas $90 \%$ of the WT mice (90 out of 100$)$ remained alive $(P<0.01)$. Interestingly, mortality was significantly higher in male ( $84 \%$ or 42 out of 50 ) compared to female TSP-2 KO mice ( $26 \%$ or 13 out of 50 ) ( $P<0.01$; Figure $1 \mathrm{~A})$. TSP-2 transcript and protein levels were significantly increased (respectively, 3.4 fold and 3.6 fold) in older compared to young TSP-2 WT hearts (Table 1 and Figure 1B). Moreover, immunoblotting revealed that TSP-2 protein levels were more elevated in older female compared to older male WT hearts (Figure 1B). Immunohistochemical analysis confirmed that TSP-2 protein expression was low in young WT hearts (Figure $1 \mathrm{C}$ ), but abundantly present and primarily located in the ECM surrounding the cardiomyocytes in both older female and male TSP-2 WT hearts (Figure 1C).

To investigate whether the absence of TSP-2 resulted in compensatory changes in expression of other TSPs before the onset of heart failure, transcript and/or protein levels of TSP-1, -3 and -4 were determined in the hearts of young and older TSP-2 KO and WT mice (Table 1). Compared to WT hearts, a clear trend towards increased TSP-4 transcript levels was observed in TSP-2 KO hearts ( $P=0.08$ at young age, $P=0.07$ at older age, Table 1). However, no significant differences in TSP-1, -3 and -4 transcript levels were noted between WT and KO hearts at both young and older age (Table 1). Concordantly, TSP-1 and -3 immunoblotting did not reveal any significant differences between the TSP-2 WT and KO hearts (Table 1).

Further analysis of the aged hearts revealed that the increased mortality was due to a progressive and severe dilated cardiomyopathy, with increasing cardiac fibrosis and dilatation with aging in TSP-2 KO mice (Figure 1D through 1K, Table 2 and 3). In concordance, echocardiographic analysis showed depressed systolic function and increased cardiac dilation in surviving TSP-2 KO compared to older WT mice (Table 2). Cardiac fibrosis, dysfunction and dilation were more pronounced in the older male compared with older female TSP-2 KO hearts (Figure 1D through $1 \mathrm{~K}$, Table 2 and 3). Increased cardiac failure in TSP-2 KO mice was further validated by an increased lung to body weight ratio compared with WT mice at 60 weeks (Table 2). At young age, cardiac function and dimensions did not differ in TSP-2 KO compared with agematched WT animals (Table 2). 

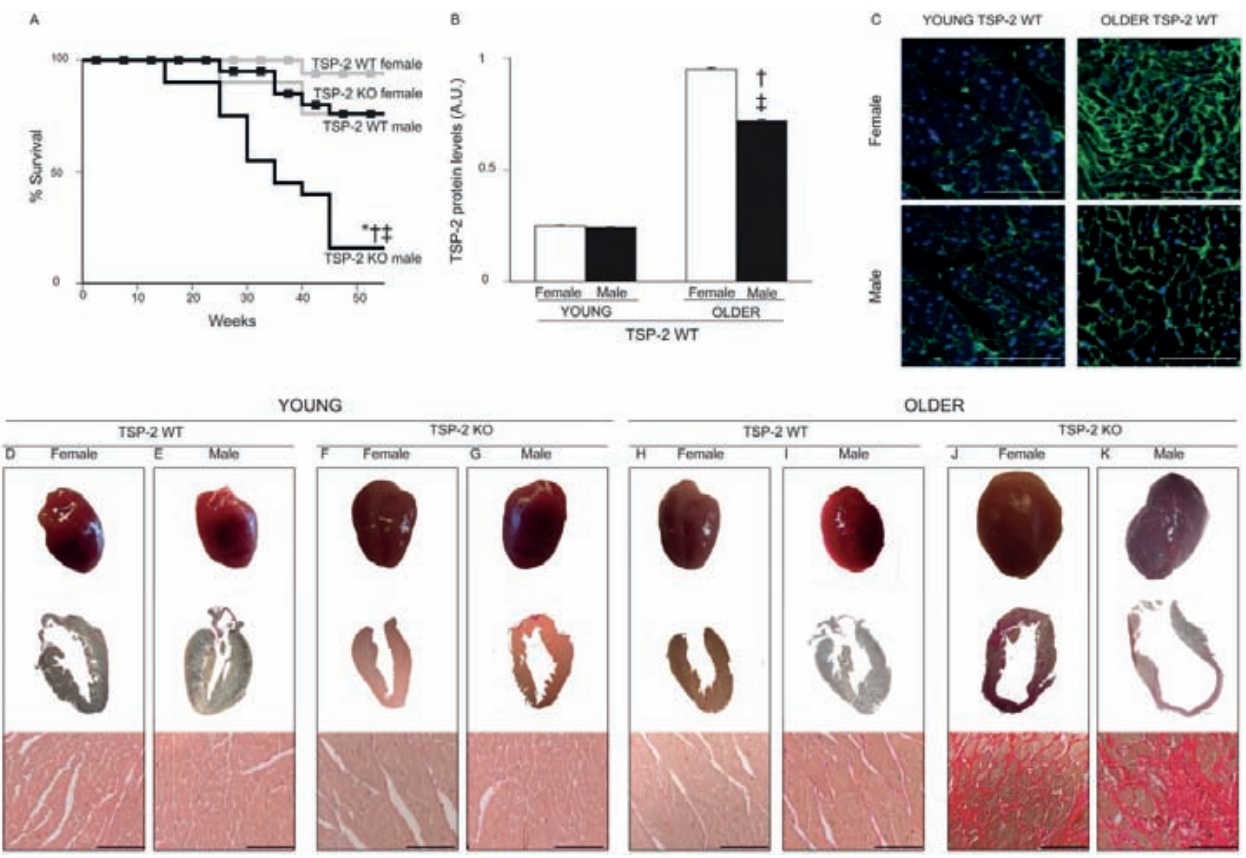

YOUNG
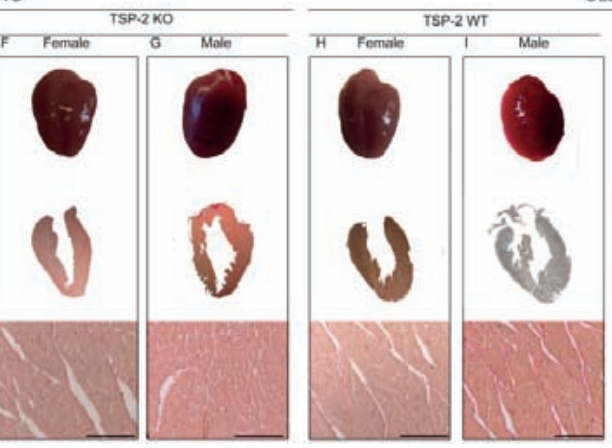

OLDER

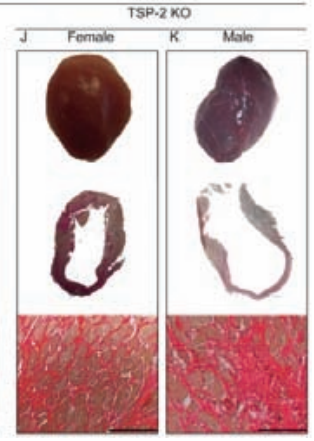

Figure 1. Age-induced dilated cardiomyopathy and increased mortality in the absence of TSP-2 (A) Survival curves of TSP-2 KO and WT mice. (B) Immunoblotting of TSP-2 in WT hearts. (C) Increased TSP-2 immunofluorescent staining in older WT hearts. (D through K) Upper part: Macroscopic hearts and sirius red staining respectively from young and older, male and female TSP-2 WT and KO mice. See also Supplemental figure $1{ }^{*} P<0.01$, TSP-2 KO mice versus TSP-2 WT mice within the same gender and age; $+P<0.01$, older TSP-2 mice versus younger TSP-2 mice within the same genotype; $¥ P<0.01$, TSP-2 female mice versus TSP-2 male mice with the same genotype within the same age-group. Bars $=100 \mu \mathrm{m}$ (C through $\mathrm{K}$ ).

\section{Structural features of accelerated aging of the heart in TSP-2 KO mice}

To study the underlying mechanisms of the age-related cardiomyopathy, the morphological features of cardiomyocytes, the interstitial matrix and the vessels, including markers of apoptosis and cell stress, were examined within the surviving male and female TSP2 WT and KO hearts at young ( 8 to 12 weeks), intermediate (25 to 30 weeks) and older (50 to 60 weeks) age.

Histopathological analysis revealed a progressive decrease in cardiomyocyte density and increase in scar-forming fibrosis with advanced age in the male and female TSP-2 KO hearts, but not in aged WT hearts (Figure 1D through 1K, Tabel 3, Supplemental figure 1). No differences were seen at young age. Cardiomyocyte dropout was further indicated by a significantly reduced left ventricular weight to body weight ratio in older TSP-2 KO mice, whereas this ratio did not differ between young WT and TSP-2 KO mice (Figure 1D through 1K, Table 3). Myocyte stress in older TSP-2 KO hearts was confirmed by desmin staining ${ }^{7}$, revealing a clear desmin disorganization (Figure 2A). Ubiquitin, a marker of cell stress, and p16, an indicator of aging ${ }^{8}$, were significantly increased in older TSP-2 KO hearts (Figure 2B and 2C, Table 3). 
Table 1. Transcript and protein levels of TSPs, oxidative stress, and inflammatory markers

\begin{tabular}{|c|c|c|c|c|}
\hline & \multicolumn{2}{|c|}{ Young } & \multicolumn{2}{|c|}{ Older } \\
\hline & WT $(n=5)$ & $\mathrm{KO}(\mathrm{n}=5)$ & WT $(n=5)$ & $\mathrm{KO}(\mathrm{n}=5)$ \\
\hline \multicolumn{5}{|l|}{ TSPs } \\
\hline \multicolumn{5}{|c|}{ Relative mRNA expression } \\
\hline TSP-1 & $1.2 \pm 0.37$ & $1.9 \pm 0.53$ & $1.1 \pm 0.35$ & $1.5 \pm 0.43$ \\
\hline TSP-2 & $1.0 \pm 0.10$ & $<0.1$ & $3.4 \pm 0.55^{\dagger}$ & $<0.1^{*}$ \\
\hline TSP-3 & $1.1 \pm 0.23$ & $2.2 \pm 0.35$ & $1.3 \pm 0.49$ & $1.7 \pm 0.27$ \\
\hline TSP-4 & $1.1 \pm 0.28$ & $5.5 \pm 2.1$ & $1.1 \pm 0.35$ & $5.8 \pm 2.7$ \\
\hline \multicolumn{5}{|c|}{ Protein levels, $\mathrm{AU}$} \\
\hline TSP-1 & $0.52 \pm 0.03$ & $0.62 \pm 0.05$ & $0.55 \pm 0.10$ & $0.60 \pm 0.13$ \\
\hline TSP-2 & $0.23 \pm 0.01$ & $<0.1^{*}$ & $0.82 \pm 0.05^{+}$ & $<0.1^{*}$ \\
\hline TSP-3 & $0.54 \pm 0.04$ & $0.59 \pm 0.09$ & $0.61 \pm 0.03$ & $0.66 \pm 0.15$ \\
\hline \multicolumn{5}{|c|}{ Oxidative stress } \\
\hline \multicolumn{5}{|c|}{ Relative mRNA expression, AU } \\
\hline CAT1 & $1.7 \pm 1.0$ & $1.6 \pm 0.9$ & $2.9 \pm 0.2^{+}$ & $2.1 \pm 0.1^{*+}$ \\
\hline SOD2 & $1.0 \pm 0.1$ & $0.71 \pm 0.1$ & $6.6 \pm 1.5^{+}$ & $6.5 \pm 1.2^{+}$ \\
\hline GXP1 & $1.0 \pm 0.01$ & $0.99 \pm 0.7$ & $1.0 \pm 0.5^{*}$ & $1.0 \pm 0.4$ \\
\hline \multicolumn{5}{|l|}{ Inflammation } \\
\hline \multicolumn{5}{|c|}{ Protein levels, pg.mL } \\
\hline TGF- $\beta$ & $4.7 \pm 1.0$ & $6.2 \pm 1.1$ & $5.0 \pm 0.9$ & $4.6 \pm 0.8$ \\
\hline \multicolumn{5}{|c|}{ Relative mRNA expression, $\mathrm{AU}$} \\
\hline TGF- $\beta$ & $1.0 \pm 0.2$ & $1.0 \pm 0.9$ & $0.15 \pm 0.05^{\dagger}$ & $0.2 \pm 0.02^{*+}$ \\
\hline IL-1 beta & $<0.05$ & $<0.05$ & $1.0 \pm 0.5^{+}$ & $4.6 \pm 0.2^{*+}$ \\
\hline IL-6 & $<0.05$ & $<0.05$ & $1.0 \pm 0.2$ & $3.8 \pm 0.4^{*+}$ \\
\hline IL-12 & $1.0 \pm 0.9$ & $1.0 \pm 0.5$ & $0.96 \pm 0.05^{+}$ & $8.7 \pm 0.9^{*+}$ \\
\hline
\end{tabular}

$\mathrm{AU}$ indicates arbitrary units.

${ }^{*} P<0.05$, TSP-2 WT versus TSP-2 KO mice within the same age group and gender.

${ }^{\dagger} P<0.05$, young versus older mice with the same genotype.

Electron microscopy substantiated cardiomyocyte stress and a disorganization of the extracellular matrix in older TSP-2 KO compared to WT hearts, whereas myocytes and matrix were normal in younger TSP-2 KO and WT hearts (Figure 2E and 2F). Cardiomyocytes of old TSP-2 KO hearts showed mitochondrial enlargement and lysis of myofilaments (Figure 2E and 2F).

Decreased cardiac mass was not due to significant changes in myocyte crosssectional area of older TSP-2 KO hearts compared to age-matched WT hearts (Table 3). Myocyte death and fibrosis were not caused by differences in vascularity (Table 3 ). 


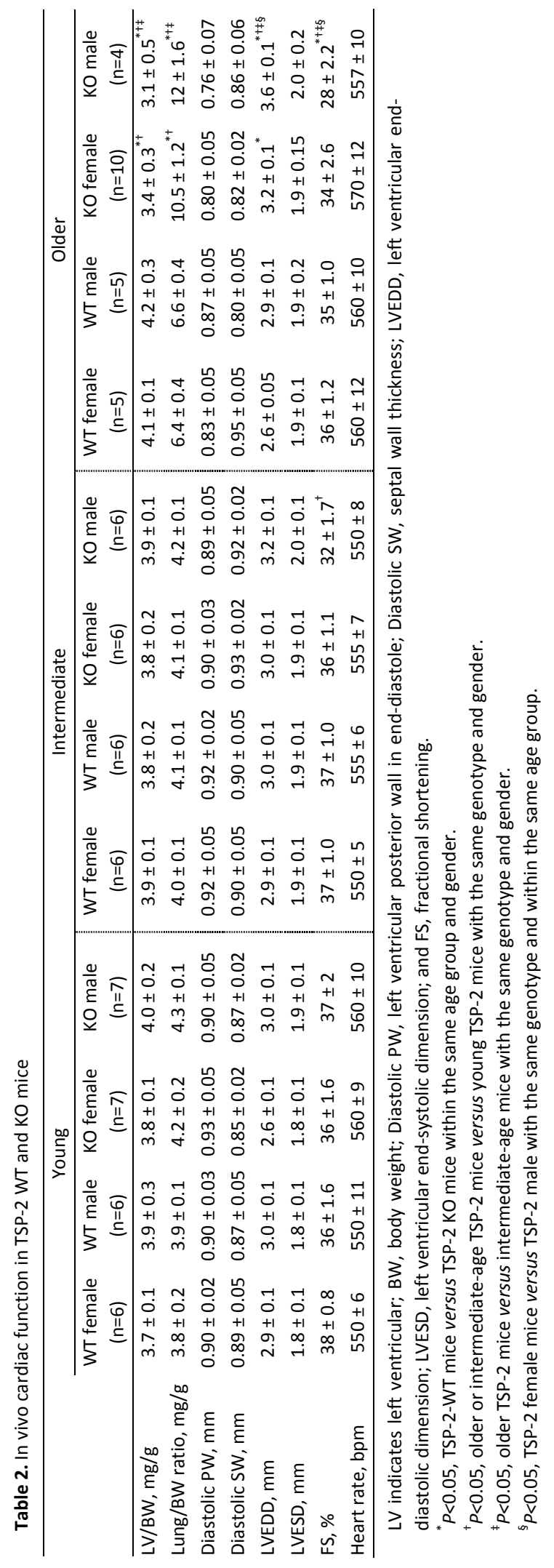




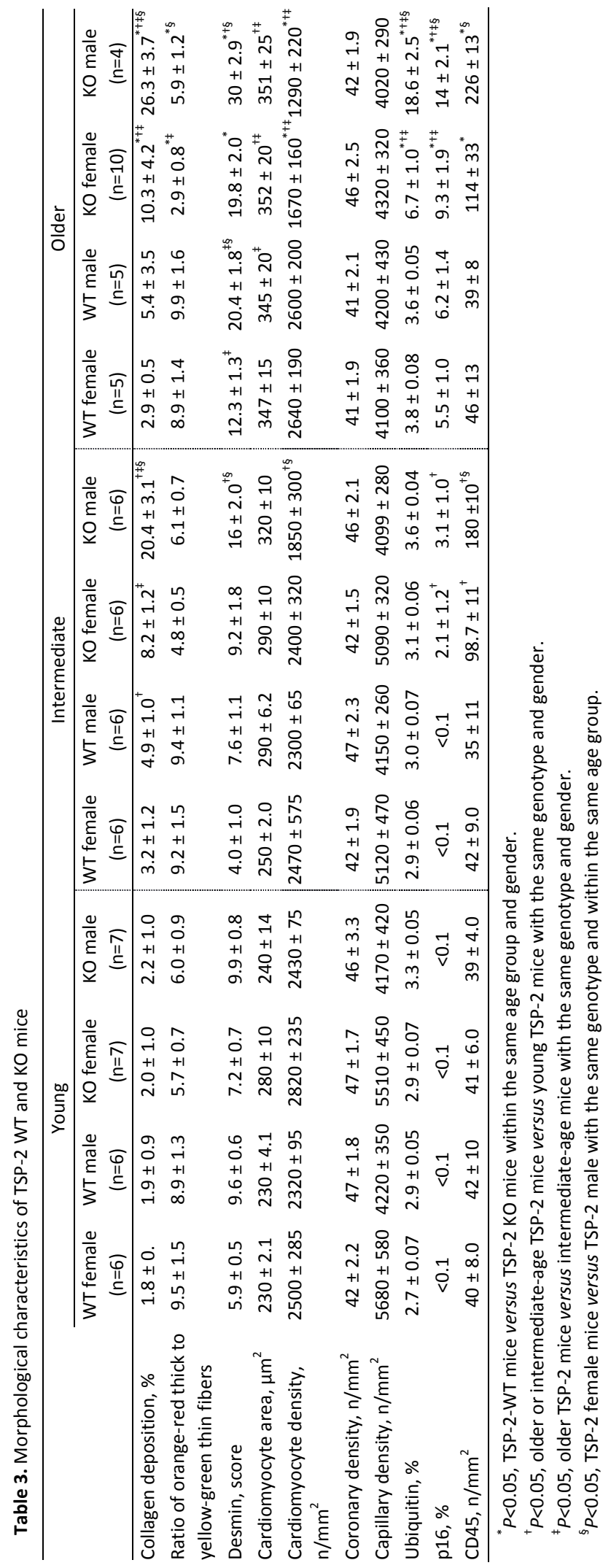



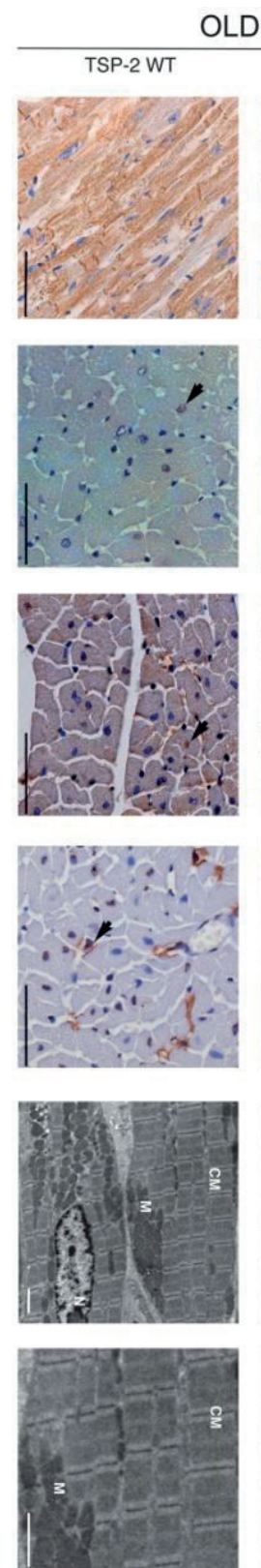
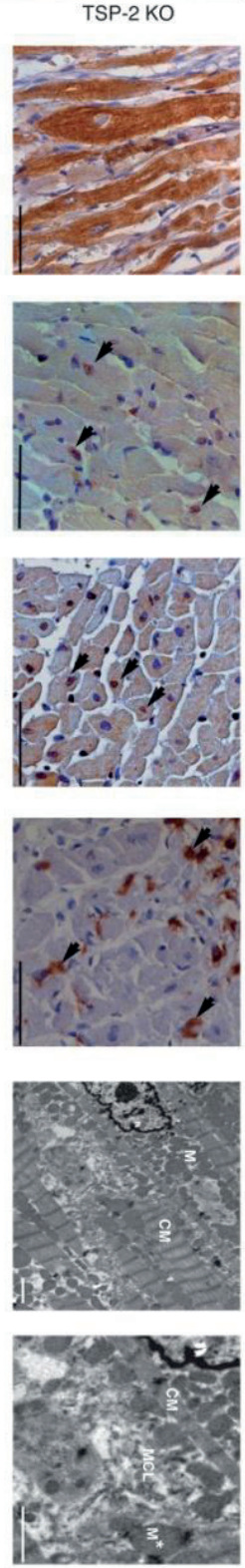
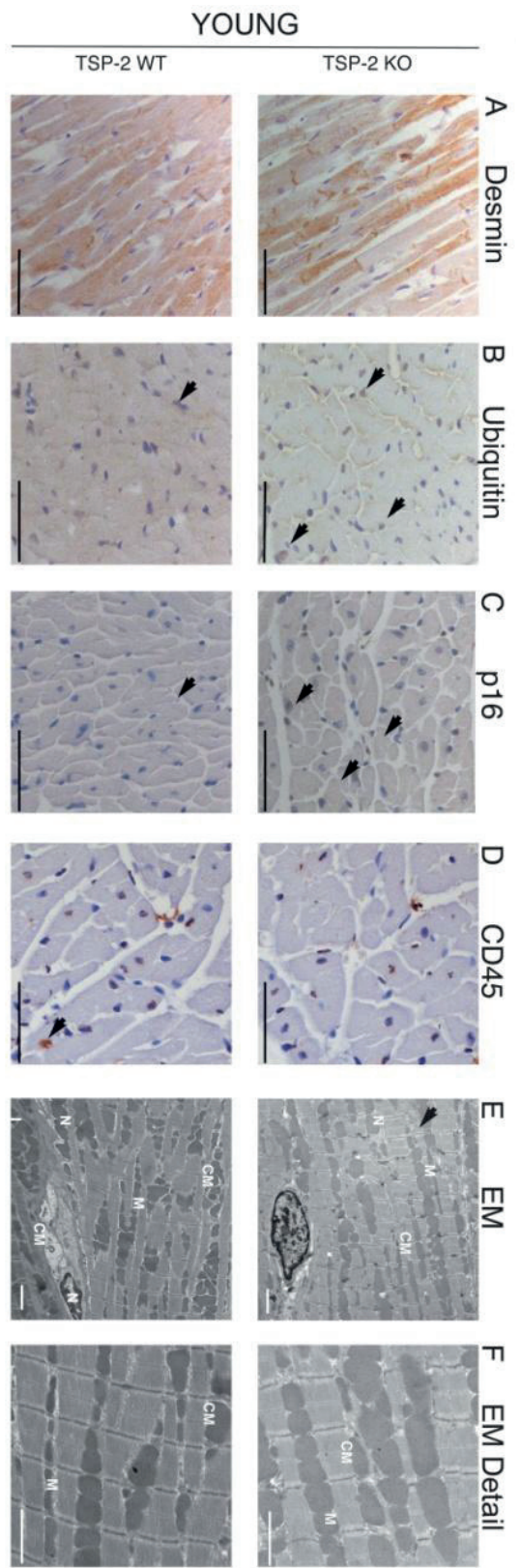

Figure 2. Structural features of accelerated aging of the hearts in TSP-2 KO mice. Histological and ultrastructural analyses of young and older male TSP-2 KO and WT hearts. (A) Desmin and (B) ubiquitin, 2 markers of cell stress; (C) p16, an indicator of aging; and (D) infiltrating CD45-positive leukocytes were increased in older TSP-2 KO hearts but did not differ at younger age. (E through F) Electron microscopic analysis revealed increased myocytolitic damage, and mitochondrial dilatation in older TSP-2 KO compared with WT hearts. Bars $=50 \mu \mathrm{m}(A$ through $D), 2 \mu \mathrm{m}(E)$, and $1 \mu \mathrm{m}(\mathrm{F}) . \mathrm{N}$ indicates nucleus; $\mathrm{M}$, mitochondria; $\mathrm{M}^{*}$, dilated mitochondria; $\mathrm{CM}$, cardiomyocyte; and $\mathrm{MCL}$, myocytolitic damage. 


\section{AAV9-TSP-2 treatment of TSP-2 KO mice prevents accelerated aging-induced cardiomyopathy}

To determine whether the age-related cardiomyopathy in TSP-2 KO mice could be rescued by postnatal gene transfer of TSP-2, we treated young male and female TSP-2 KO mice with AAV9-TSP-2 compared to a control AAV9-GFP (Figure 3A through 3F).

Importantly, AAV9-TSP-2 blunted the mortality observed in the older control AAV9-GFP-treated TSP-2 KO mice (68\%, 24 out of 35; 3 out of 10 females and 21 out of 25 males; Figure 3A). AAV9-TSP-2 also prevented cardiomyocyte dropout, fibrosis, cardiac dilatation and dysfunction, still present in the aged control-treated TSP-2 KO mice (Figure $3 \mathrm{~A}$ through $3 \mathrm{~F}$ and Table 4).

Together, these data confirm that post-natal TSP-2 expression is essential for maintaining the myocardial architecture and function with age.

Table 4. AAV9-mediated rescue of TSP-2 KO mice

\begin{tabular}{|c|c|c|c|c|}
\hline & \multicolumn{2}{|c|}{ AAV9-GFP } & \multicolumn{2}{|c|}{ AAV9-TSP-2 } \\
\hline & KO female $(n=7)$ & KO male $(n=4)$ & KO female $(n=19)$ & KO male $(n=10$ \\
\hline \multicolumn{5}{|l|}{ Functional measurements } \\
\hline $\mathrm{LV} / \mathrm{BW}, \mathrm{mg} / \mathrm{g}$ & $3.6 \pm 0.1$ & $2.9 \pm 0.2$ & $3.8 \pm 0.1$ & $3.9 \pm 0.1^{*}$ \\
\hline Lung/BW ratio, mg/g & $9.5 \pm 1.3$ & $10.9 \pm 1.1$ & $4.1 \pm 0.2^{*}$ & $4.1 \pm 0.1^{*}$ \\
\hline Diastolic PW, mm & $0.81 \pm 0.02$ & $0.77 \pm 0.05$ & $0.96 \pm 0.01^{*}$ & $0.98 \pm 0.01^{*}$ \\
\hline Diastolic SW, mm & $0.85 \pm 0.04$ & $0.85 \pm 0.06$ & $0.94 \pm 0.01^{*}$ & $0.94 \pm 0.01^{*}$ \\
\hline LVEDD, $\mathrm{mm}$ & $3.1 \pm 0.2$ & $3.5 \pm 0.2^{\dagger}$ & $2.7 \pm 0.1^{*}$ & $2.7 \pm 0.1^{*}$ \\
\hline LVESD, $\mathrm{mm}$ & $1.90 \pm 0.2$ & $1.99 \pm 0.3$ & $1.70 \pm 0.2^{*}$ & $1.70 \pm 0.2^{*}$ \\
\hline $\mathrm{FS}, \%$ & $33 \pm 1.2$ & $29 \pm 0.9^{\dagger}$ & $38 \pm 0.2^{*}$ & $37 \pm 0.7^{*}$ \\
\hline Heart rate, bpm & $555 \pm 5.0$ & $560 \pm 2.0$ & $550 \pm 5.0$ & $555 \pm 7.0$ \\
\hline \multicolumn{5}{|l|}{ Histopathology } \\
\hline Collagen deposition, $\%$ & $9.5 \pm 0.5$ & $24.3 \pm 0.3^{+}$ & $1.2 \pm 0.2^{*}$ & $1.4 \pm 0.1^{*}$ \\
\hline Cardiomyocyte density, $\mathrm{n} / \mathrm{mm}^{2}$ & $1630 \pm 170$ & $1330 \pm 255^{+}$ & $2620 \pm 185^{*}$ & $2560 \pm 190^{*}$ \\
\hline
\end{tabular}

LV indicates left ventricular; BW, body weight; Diastolic PW, left ventricular posterior wall in end-diastole; Diastolic SW, septal wall thickness; LVEDD, left ventricular end-diastolic dimension; LVESD, left ventricular end-systolic dimension; and FS, fractional shortening.

${ }^{*} P<0.05$, AAV9-GFP-treated versus AAV9-TSP-2 treated TSP-2 KO mice of the same gender.

${ }^{\dagger} P<0.05$, TSP-2 female mice versus TSP-2 male mice within the same treatment group. 


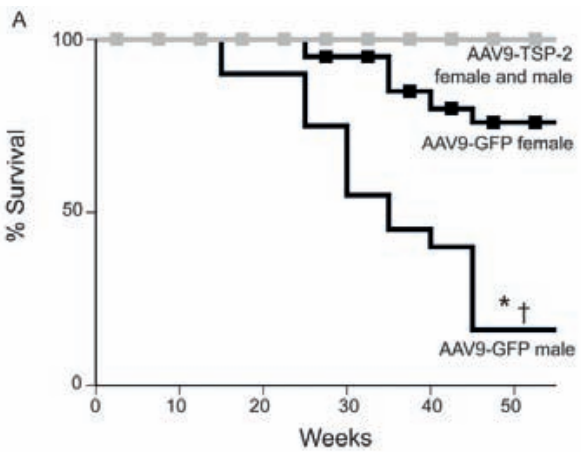

B
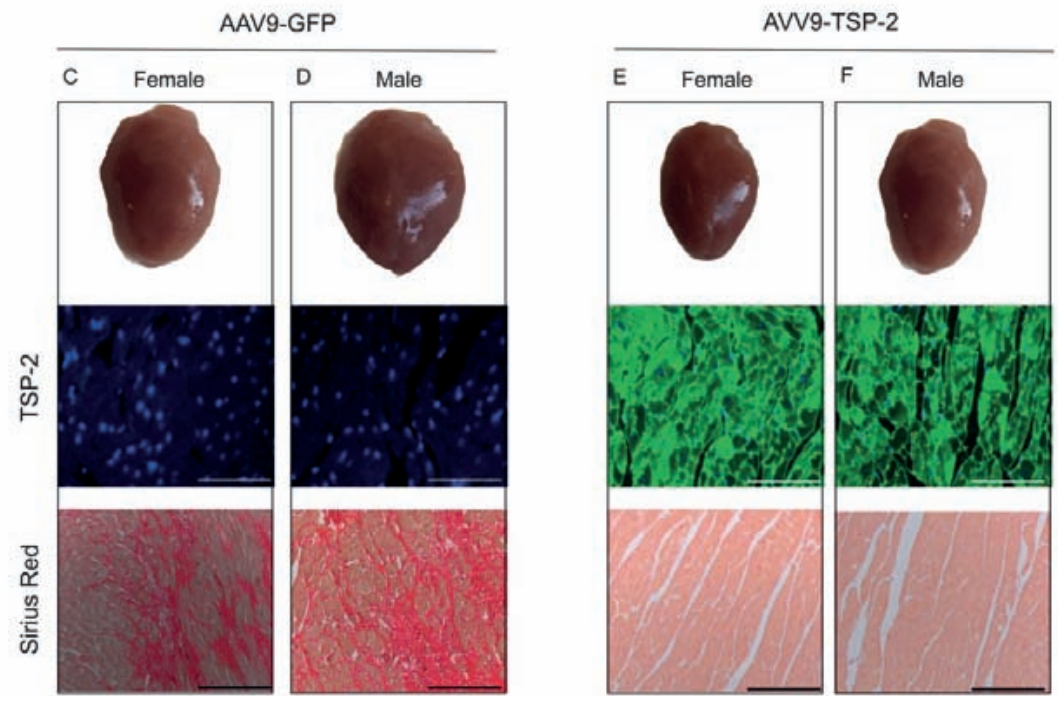

Figure 3. AAV9-TSP-2 treatment of TSP-2 KO mice prevents accelerated aging-induced cardiomyopathy. (A) Survival curve of male and female AAV9-GFP- and AAV9-TSP-2-treated TSP-2 KO mice. (B through F) At 60 weeks of age, both TSP-2 immunoblotting (B) and immunofluorescent staining ( $C$ through $F$, middle, green) confirmed a significant and widespread cardiac TSP-2 protein expression in AAV9-TSP-2 (B, E and F) vs AAV9-GFP-treated TSP-2 KO mice ( $B$ through $D$; for $B, n=5$ per group). ( $C$ through $F$, upper part) Blunted cardiac dilation in AAV9-GFP- (C-D) vs AAV9-TSP-2-treated TSP-2 KO mice (E and F). (C through F, lower part) Sirius red staining revealing blunted cardiac fibrosis in AAV9-TSP-2-treated TSP-2 KO mice. ${ }^{*} P<0.05$, AAV9-GFP-treated versus AAV9-TSP-2-treated TSP-2 KO mice with the same gender; $\uparrow P<0.05$, TSP-2 female mice versus TSP-2 male mice with the same AAV9 treatment. Bars $=100 \mu \mathrm{m}$

\section{Increased inflammation in older TSP-2 KO hearts}

Next, we investigated whether a prolonged low-grade inflammation in response to aging-associated free radical generation could underlie the age-related cardiomyopathy observed in TSP-2-KO mice. ${ }^{9}$

Cardiac aging and progressive myocyte stress in the absence of TSP- 2 were accompanied by increased inflammation. The number of CD45-positive inflammatory 
cells progressively increased with age, reaching significance in TSP-2 KO compared with WT hearts at older age (Figure 2D and Table 3), but no significant differences were noticed at young age (Figure 2D and Table 3). Inflammation was more pronounced in older male compared with female TSP-2 KO mice (Figure 2D and Table 3). In concordance, cardiac transcript levels of TGF- $\beta 1$, interleukin (IL) $-1 \beta$, IL-6, and IL-12 significantly increased with age and in older TSP-2 KO compared with WT hearts (Table 1). The activation of TGF- $\beta 1$ did not differ (Table 1 ). In addition, transcript levels of enzymes influencing the oxidation status of the heart, including super oxide dismutase-2 and glutathione peroxidase- 1 did not significantly differ between TSP-2 WT and KO hearts (Table 1), whereas catalase- $1^{10}$ was significantly higher in older WT compared to KO hearts (Table 1). In conclusion, age-related cardiomyopathy in the absence of TSP-2 is due to progressive cardiomyocyte stress and dropout, accompanied by increased inflammation and scar-forming reparative fibrosis.

\section{Higher MMP-2- and lower tissue-transgulatiminase-2- activity in TSP-2 KO mice}

Previous studies have shown that a lack of TSP-2 results in an increase in MMP-2 activity, which in turn inactivates tissue-transglutaminase-2. The latter enzyme is involved in collagen cross-linking, and may protect against cardiac dilation and dysfunction. ${ }^{11,12}$ To investigate whether the increased cardiac dilation in the absence of TSP-2 may relate to increased MMP-2 and consequently decreased tTG activity, their activity levels were measured in young and older TSP-2 KO and WT hearts.

Whereas no significant differences were noted in TSP-2 KO hearts, MMP-2 zymographic activity significantly decreased in TSP-2 WT hearts with progressing age (Figure 4A). MMP-2 activity levels were significantly lower in young KO compared with WT hearts, but significantly higher in older KO compared with older WT hearts. MMP-9 zymographic activity did not differ significantly in TSP-2 KO compared with WT hearts at both young or old age (Figure 4B).

Decreased MMP-2 activity with progressing age in TSP-2 WT hearts was paralleled by increased tTG-activity (Figure $4 \mathrm{C}$ ). Concordantly, increased MMP-2 activity in older TSP-2 KO compared with older WT hearts was paralleled by reduced activity of tTG-2, and a decrease in $\varepsilon$-lysyl $\gamma$-glutaminyl cross-links (Figure 4D and 4E). A significant decrease of tTG-2 activity in TSP-2 KO compared with WT hearts was also observed at younger age (Figure $4 \mathrm{~A}$ and $4 \mathrm{C}$ ).

Sirius red-polarization microscopy revealed mainly well-aligned, thick and tightly packed orange-red-collagen fibers in young and older WT hearts. Loosely assembled yellow-green collagen fibers predominated the older TSP-2 KO hearts (Figure 4F and 4G, Table 3). Thus, increased MMP-2, decreased tTG-2 activity, and impaired collagen maturation in the absence of TSP-2 contributed to increased cardiac dilation with aging. 

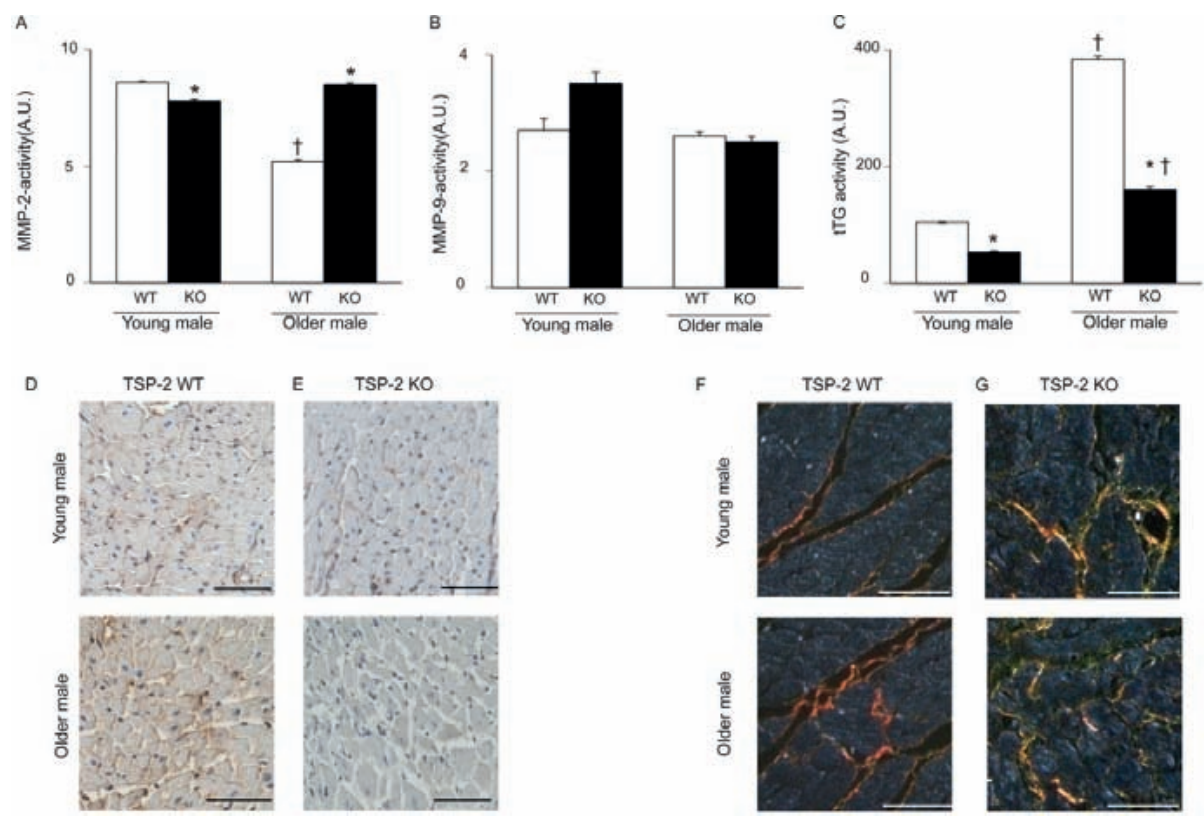

Figure 4. Higher MMP-2 and lower tTG-2 activity in TSP-2-KO mice. (A) Significantly increased MMP-2 zymographic acitvity in older TSP-2 KO hearts. (B) No significant differences in MMP-9 activity in TSP-2 WT vs KO mice. (C) Significantly decreased levels of tTG-activity in TSP-2 KO aged hearts ( $n=7$ per group). (D and E) Decreased $\varepsilon$-lysyl- $\gamma$-glutaminyl cross-linking staining in older TSP-2 KO hearts (E and D, respectively; lower panel). ( $F$ and G) Sirius red-polarization microscopy revealing more loosely assembled (yellow-green) collagen fibers predominating in older TSP-2 KO hearts. ${ }^{*} P<0.05$, TSP-2 WT mice versus TSP-2 KO mice but within the same age-group; $+P<0.05$, young TSP- 2 mice versus older TSP- 2 mice but with the same genotype.

\section{Loss of TSP-2 results in impaired activation of Akt in vivo and in vitro}

The Akt pathway is centrally involved in promoting myocyte survival, and its activation protects against cardiac injury, fibrosis and failure. ${ }^{13,14}$ TSP-2 contains two conserved domains that are able to activate the Akt pathway, namely the CD47-binding site within its $\mathrm{C}$-terminal domain, and the $\mathrm{N}$-terminal $\beta 1$-integrin recognition site that is proximal to the CD36-binding domain. ${ }^{15}$ Therefore, we investigated whether these interactions might contribute to the cardiomyocyte survival pathway.

First, a significantly reduced activation of Src and Akt was demonstrated in TSP-2 KO compared with WT hearts (Figure 5A and 5B). Immunoblotting for ILK and in vitro kinase activity assays were performed to determine the relative level and activity of ILK in WT and TSP-2 KO hearts. However no differences in ILK activity could be detected in the hearts of TSP-2 WT and KO mice. (Supplemental figure 2). Next, an interaction of TSP-2 with the Src/Akt pathway was confirmed in cardiac myocytes in vitro. The use of a lentiviral short-hairpin RNA (shRNA) produced an $80 \%$ knockdown of TSP-2 and resulted in significantly reduced phosphorylation of Src and Akt (Figure 5C through 5G), whereas a control, unrelated short-hairpin RNA lentiviral vector did not alter their phosphorylation status. 

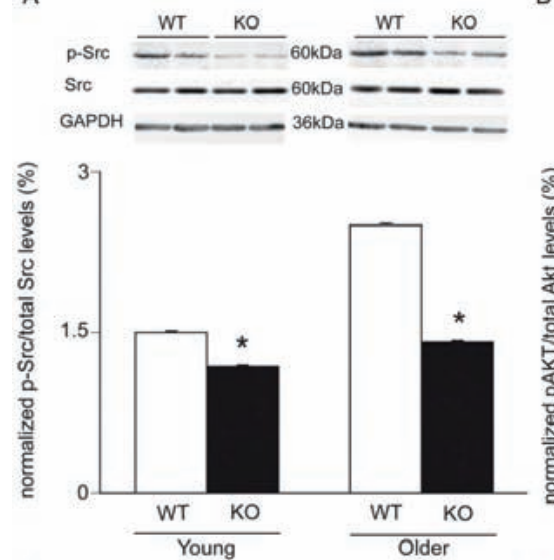

C

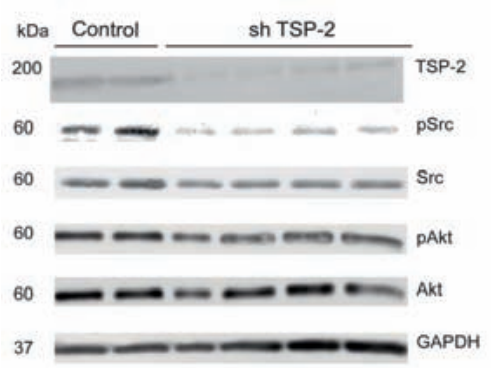

$\mathbf{F}$

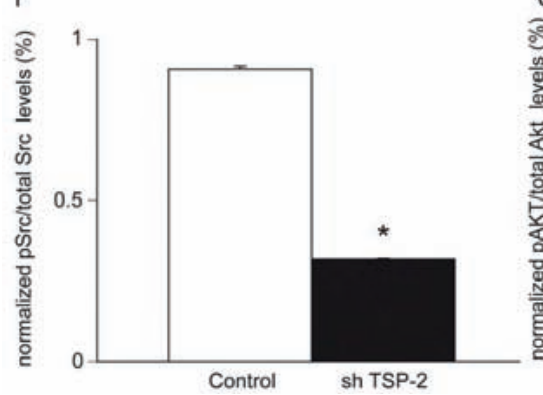

D
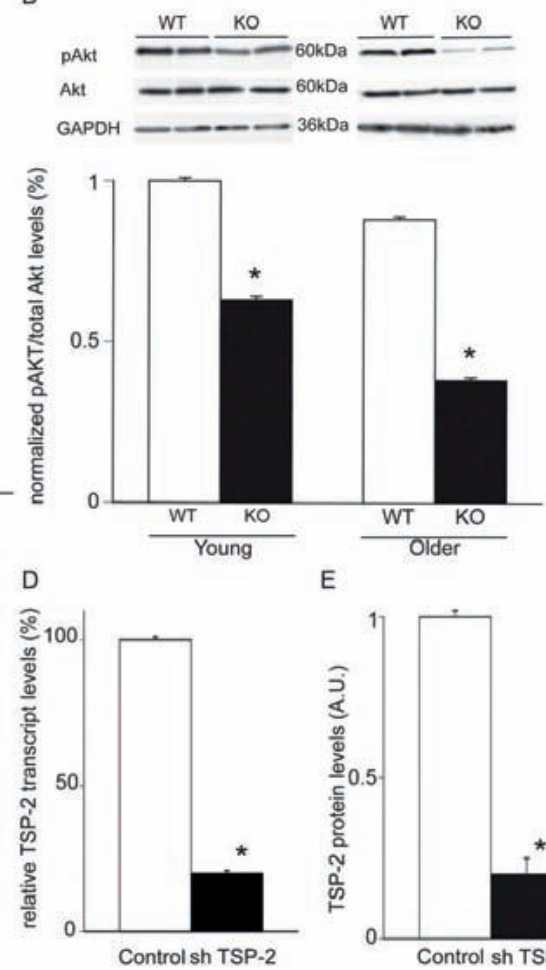

E
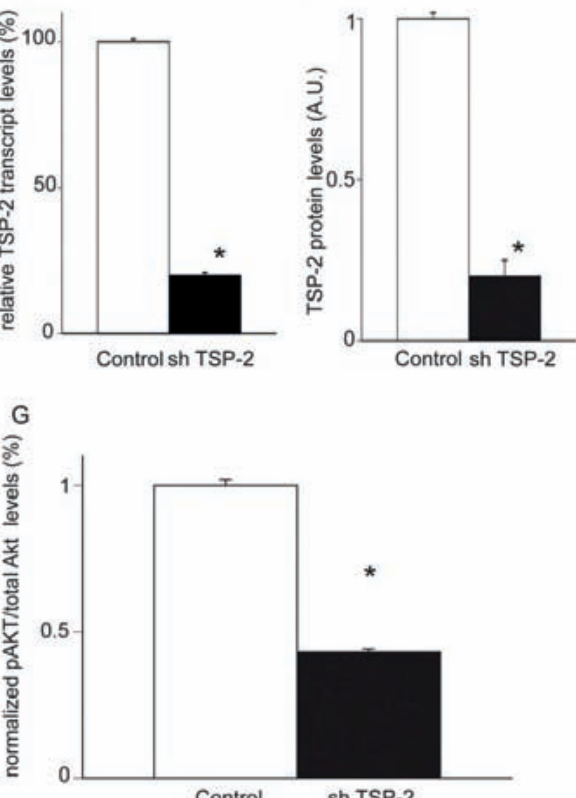

Figure 5. Loss of TSP-2 results in impaired Src/Akt-dependent cardiomyocyte survival in vivo and in vitro. (A and B) Src protein levels and ratio of phosphorylated Akt to total Akt were significantly decreased in TSP2 KO vs age-matched WT littermates ( $n=7$ per group). (C through G) In vitro experiments showing that knockdown for TSP-2 results in a significant reduction of Src and Akt phosphorylation in neonatal rat cardiomyocytes. All protein levels were normalized for GAPDH.

Finally, monoclonal blocking antibodies against either the CD47- $\left(\alpha-\mathrm{TSP}^{\mathrm{CD} 47}\right)$ or the CD36- $\left(\alpha-\right.$ TSP $\left.^{\mathrm{CD} 36}\right)$ binding sites of TSP were administered to neonatal rat cardiomyocytes in vitro (Figure 6A through $6 \mathrm{C}$ ). Blocking of the TSP/CD47 interaction resulted in reduced phosphorylation of Src and Akt (Figure 6E through 6G) and caused obvious myocyte stress compared to control treatment. Here, myocyte stress was indicated by changes in the cytoskeletal organization as revealed by altered phalloidin staining, a 
significantly decreased cardiomyocyte density and increased protein expression of activated caspase-3 (Figure 6A through 6D and 6H). Blocking of the CD36-binding site of TSP, however, did not significantly alter Src- or Akt- phosphorylation, nor did it change cardiomyocyte appearance or expression of activated caspase-3 (Figure $6 \mathrm{~A}$ through $6 \mathrm{H})$.

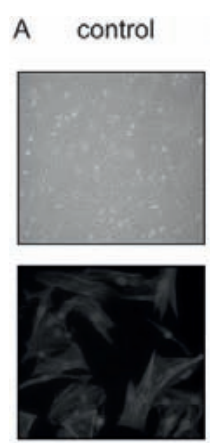

E
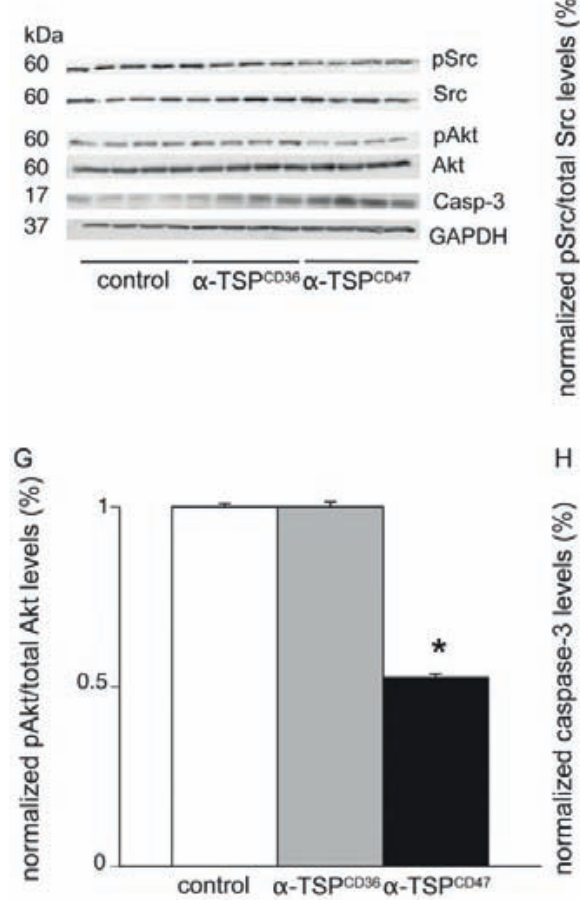

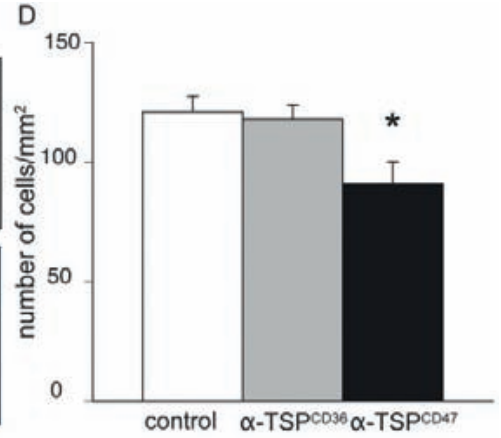

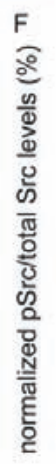
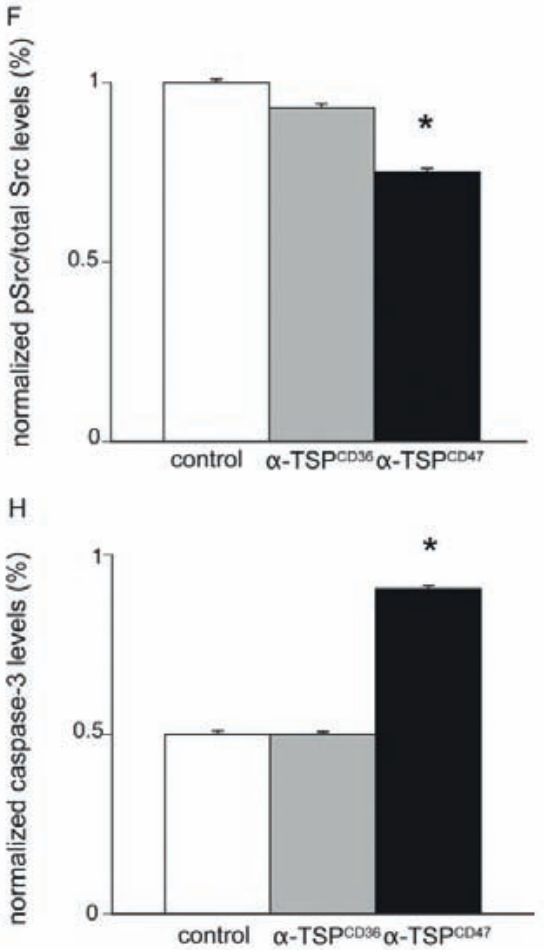

Figure 6. CD47 is involved in cardiac survival. Cell culture of neonatal rat cardiomyocytes treated with a $\alpha$ $\mathrm{TSP}^{\mathrm{CD} 47}$-blocking antibody $(\mathrm{C})$ resulted in cytoskeletal impairment and a significantly decreased number of neonatal rat cardiomyocytes at 48 hours vs $\alpha-\operatorname{TSP}^{\mathrm{CD} 36}$ (B) or the control group (untreated; A) (E through $\mathrm{H}$ ) Decreased Src and Akt phosphorylation and increased active cleaved caspase- 3 in $\alpha-\mathrm{TSP}^{\mathrm{CD} 47}$ treated neonatal rat cardiomyocytes ( $\mathrm{F}$ through $\mathrm{H} ; \mathrm{n}=6$ per treatment) $* P<0.05$. 

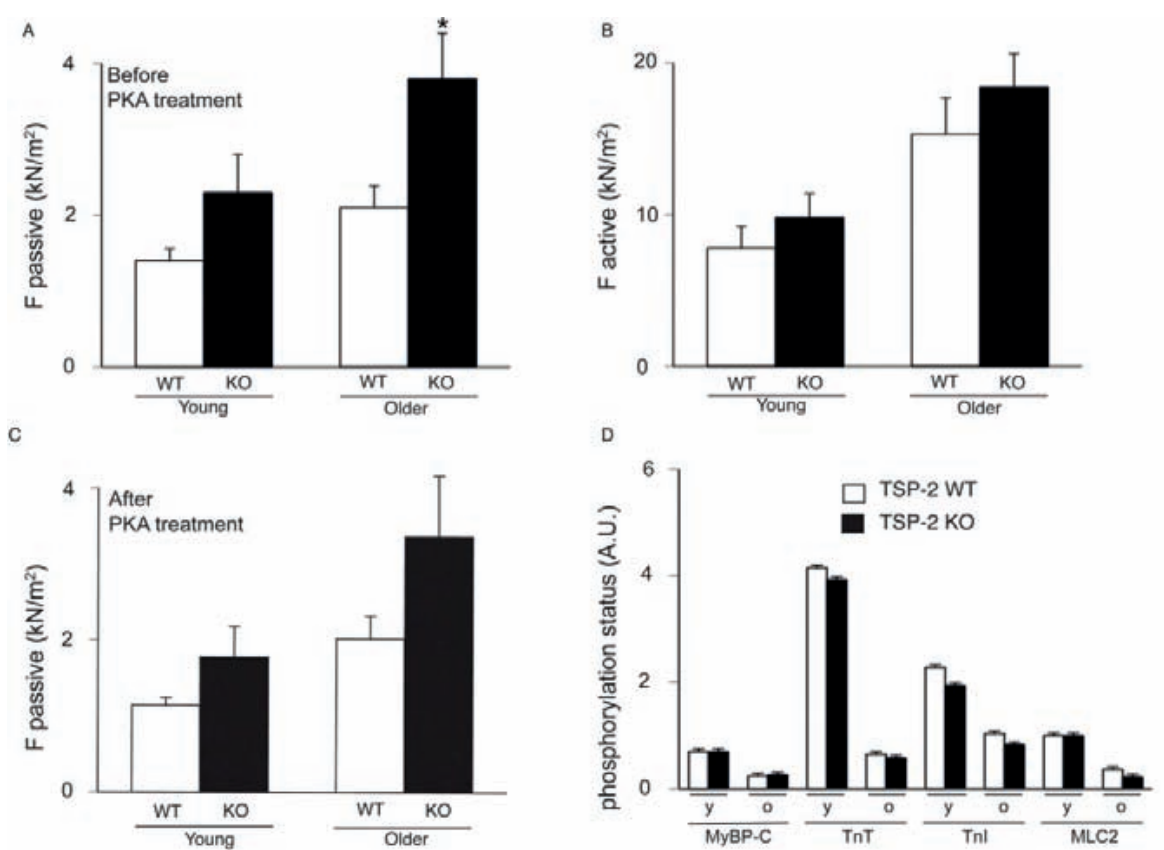

Figure 7. The absence of TSP-2 affects diastolic function of individual isolated cardiomyocytes. Passive forces (A) are significantly increased but active forces (B) are similar in TSP-2 KO vs WT myocytes. (C) Exogenous PKA treatment did not change the differences in passive forces. (D) Phosphorylation status of myosin-binding protein C (MyBP-C), Troponin T (TnT), Troponin I (Tnl), and Myosin Light Chain-2 (MLC-2) revealed no significant differences between TSP-2 WT and KO mice at young (y) and old (o) age $(n=10$ cardiomyocytes per genotype per age-group) ${ }^{*} P<0.05$, TSP-2 WT mice versus TSP-2 KO mice within the same age-group.

\section{Absence of TSP-2 affects cardiomyocyte function with aging}

To investigate the effect of the age-related cardiomyopathy on the function of surviving cardiomyocytes, active and passive forces and $\mathrm{Ca}^{2+}$ sensitivity were determined through the use of individual permeabilized cardiomyocytes isolated from young and older TSP-2 KO and WT left ventricles. Passive and active forces and $\mathrm{Ca}^{2+}$ sensitivity did not differ in young TSP-2 KO compared to WT myocytes ( $n=10$ cardiomyocytes out of 5 hearts per group; $P=N S$; Figure 7A and 7B). Forces increased with advanced age in both TSP-2 KO and WT cardiomyocytes, but active forces did not differ between TSP-2 KO and WT cardiomyocytes (Figure 7B). Passive forces were significantly higher in older TSP-2 KO compared with WT cardiomyocytes, indicating increased cardiomyocyte stiffness. Normalized force-PCA curves showed similar myofilament $\mathrm{Ca}^{2+}$ sensitivity at young and older ages in TSP-2 WT and KO mice (data not shown). Impaired diastolic function of isolated cardiomyocytes was not due to alteration in myofilament phosphorylation because treatment with the catalytic subunit protein kinase-A (PKA) provided similar effects in TSP-2 KO and WT cardiomyocytes (Figure 7C). PKA treatment resulted in non-significant reduced passive forces in young TSP-2 KO mice (passive 
force before PKA: WT, 1,4 $\pm 0.2 ; \mathrm{KO}, 2.3 \pm 0.5$ : passive force after PKA: WT, $1.1 \pm 0.1$; KO: $1.8 \pm 0.4$ ). Similarly, passive forces in the older mice did not change significantly. Phosphorylation levels of myosin binding protein c, myosin light chain 2, and troponinT and -I did not significantly differ in TSP-2 KO compared with WT hearts at both young and older ages (Figure 7D).

\section{Discussion}

With advanced medical assistance and better control of cardiovascular risk factors, the general population is getting older and many people may live to be $>90$ years of age without experiencing cardiac dysfunction. Still, little is known about the physiological survival mechanisms that help to "adapt" the heart and prevent myocyte death, fibrosis and dysfunction in response to the cumulative hemodynamic stress related to aging. An understanding of these processes may lead to new therapeutic approaches that promote the longevity of the heart, even in the absence of hypertension, diabetes, smoking or coronary disease.

We provide evidence that cardiac expression of the matricellular protein TSP-2 protects against aging related functional decline of the heart. Loss of TSP-2 resulted in progressive cardiomyocyte death, accompanied by inflammation and reparative scarforming fibrosis, all of which contributed to the development of progressive cardiac dilation and dysfunction. Importantly, postnatal AAV9-mediated transfer of TSP-2 in young TSP-2 KO mice completely rescued the progressive structural and functional decline with aging.

In view of its matrix-cell-regulating properties, a protective role for TSP-2 against age-related heart disease may be mediated by regulating outward-inward cell signaling and function, thereby affecting cardiac remodeling. First, TSP-2 seems to protect against age-related myocyte death, at least in part, by promoting the Akt-survival pathway in cardiomyocytes. ${ }^{15}$ The absence of TSP-2 in vivo or its knockdown in vitro resulted in reduced activation of Src and Akt, which are both involved in promoting myocyte survival. ${ }^{13}$ Moreover, our data indicate that the conserved C-terminal CD47binding of TSP-2 is implicated in activating the Src/Akt survival pathway in cardiomyocytes. A blocking antibody against the CD47-binding domain $\left(\alpha-\mathrm{TSP}^{\mathrm{CD} 47}\right)$, but not against the CD36-binding domain $\left(\alpha-\mathrm{TSP}^{\mathrm{CD} 36}\right)$, significantly reduced activation of Src/Akt and increased myocyte stress and apoptosis in vitro. To exclude that other upstream regulators are responsible for activating the effector protein Akt, the interaction with Integrin Linked Kinase (ILK) was investigated in vivo. ILK functions downstream and independently of phosphatidylinositol 3-kinase to phosphorylate Akt. Immunoblotting for ILK together with ILK activity measurements did not show differences in KO and WT hearts. Together, our data indicate that the effect of TSP-2 on Akt phosphorylation is Src dependent. Previous reports have shown that the Akt-pathway is regulated through several receptor and receptor-independent pathways (i.e. mechani- 
cal stretch). ${ }^{16,17}$ Concordantly, we cannot exclude that TSP-2 might also regulate myocyte survival by influencing other survival pathways, including ERK1-2 and p38MAPkinases $^{18}$, focal adhesion kinases ${ }^{19}$, or STAT3 signalling pathways. ${ }^{20}$

A protective role for TSP-2-mediated Akt activation in physiological aging, however, fits with a crucial role for Akt1 and Akt2 in physiological growth of cardiomyocytes during exercise; Akt1 or Akt2-deficient mice are not able to develop physiological hypertrophy during exercise but develop heart failure in response to pressure overload. ${ }^{13,21}$ Moreover, the implication of outside-inside signaling and more specifically Akt activation in promoting (cardio)myocyte survival has also been demonstrated for other matricellular proteins. Periostin- ${ }^{22}$ and CCN-1- $\left(\right.$ Cyr-61) ${ }^{23}$-deficient mice have impaired myocyte survival from reduced activation of the Akt-survival pathway.

Beside its role in outside-inside signaling, TSP-2 also regulates extracellular matrix remodeling. It forms a complex with pro-MMP-2 and TIMP-2 which is then internalized by the low density lipoprotein-related scavenger receptor LRP1 and thereby downregulates total MMP-2 activity. ${ }^{24}$ MMP-2 in turn decreases total tTG activity ${ }^{11}$ and may diminish the binding of TSP to CD47, thereby decreasing its downstream activity. ${ }^{25}$ Aging in mice lacking TSP-2 results in increased MMP-2 activity, decreased tTG activity, and reduced collagen cross-linking, all contributing to increased cardiac dilation and dysfunction. ${ }^{2}$ In concordance, Agah et al. ${ }^{26}$ previously reported increased dermal TSP-2 expression together with decreased clearance of MMP-2 from the pericellular environment, as a function of age in TSP-2 WT mice.

TSP-2 was also shown to regulate angiogenesis and inflammation., ${ }^{96,27}$ Here, we show that myocyte death and fibrosis with progressing age were not caused by differences in vascularity. However, inflammation was increased in the absence of TSP-2 in the older but not in the younger hearts. Both progressive cardiomyocyte death and the loss of the CD47-mediated anti-inflammatory effect of TSP- $2^{9}$ might have contributed to this enhanced inflammatory influx. Whether the increased inflammatory response in aged TSP-2 KO mice mainly acts to remove the necrotic cardiomyocytes or whether the increased inflammation represents a primary contributing factor that aggravates the cardiomyocyte injury, fibrosis and the age-related cardiomyopathy remains to be resolved.

The morphological and functional features of this age-related cardiomyopathy and mortality mainly occurred in male KO mice. Several previous studies have shown that female mice display a lower mortality and less severe cardiac pathology compared with their male counterparts. ${ }^{28}$ Factors such as hormonal status, higher blood pressure, and increased physical activity may predispose male KO mice to the severe myocardial damage, dilation and dysfunction, and mortality compared with female mice with increasing age. ${ }^{28-30}$

Intriguingly, AAV9-mediated expression of TSP-2 in 7-week-old TSP-2 KO mice rescued this lethal phenotype, confirming that postnatal expression of TSP-2 is crucial for the normal physiological aging of the heart. These results also make it less probable that prenatal or early postnatal morphological changes in the heart of TSP-2 KO mice 
could underlie this progressive phenotype. Due to its cardiotrophic properties, AAV9mediated gene transfer rescues a severe cardiac phenotype. AAV9-mediated gene transfer deserves to be further explored as a novel therapeutic tool for the clinical application of gene therapy in human cardiac diseases. ${ }^{31}$

To study whether aging of cardiomyocytes in the absence of TSP-2 may affect their function per se, isometric force measurements were performed in isolated, permeabilized cardiomyocytes. Active forces did not differ in aged TSP-2 KO compared with WT cardiomyocytes, whereas passive forces indicative of diastolic dysfunction were increased in the surviving TSP-2 KO cardiomyocytes. These alterations were independent of exogenous protein kinase-A activity, excluding myofilament phosphorylation as the underlying cause of cardiac dysfunction of isolated cardiomyocytes. Together, these data indicate that the surviving individual cardiomyoyctes in the aged TSP-2 KO heart are characterized by increased stiffness, whereas active forces are not affected. Thus, progressive myocyte death, but not reduced contractility of individual cardiomyocytes, is responsible for the progressive cardiac dysfunction in the absence of TSP- 2 .

Our present study reveals a novel and pivotal role for TSP-2 in the protection against age-related cardiomyopathy. Decreased activation of the Src/Akt survival pathway in the absence of TSP-2, together with increased inflammation, MMP-2 activity, and decreased collagen cross-linking, all contributed to impaired cardiomyocyte survival and increased cardiac dilation and dysfunction with advanced age.

\section{Acknowledgments}

We thank Rick van Leeuwen, Wouter Verhesen, Kevin Custers and Hans Duimel for their technical support. 


\section{References}

1 Susic D, Frohlich ED. The aging hypertensive heart: a brief update. Nature clinical practice. 2008;5(2):104-110.

2 Spinale FG. Myocardial matrix remodeling and the matrix metalloproteinases: influence on cardiac form and function. Physiological reviews. 2007;87(4):1285-1342.

3 Schellings MW, Pinto YM, Heymans S. Matricellular proteins in the heart: possible role during stress and remodeling. Cardiovasc Res. 2004;64(1):24-31.

4 Bornstein P, Sage EH. Matricellular proteins: extracellular modulators of cell function. Curr Opin Cell Biol. 2002;14(5):608-616.

5 Schroen B, Heymans S, Sharma U, Blankesteijn WM, Pokharel S, Cleutjens JP, Porter JG, Evelo CT, Duisters R, van Leeuwen RE, Janssen BJ, Debets JJ, Smits JF, Daemen MJ, Crijns HJ, Bornstein P, Pinto YM. Thrombospondin-2 is essential for myocardial matrix integrity: increased expression identifies failureprone cardiac hypertrophy. Circ Res. 2004;95(5):515-522.

6 Kyriakides TR, Leach KJ, Hoffman AS, Ratner BD, Bornstein P. Mice that lack the angiogenesis inhibitor, thrombospondin 2, mount an altered foreign body reaction characterized by increased vascularity. Proc Natl Acad Sci U S A. 1999;96(8):4449-4454.

7 Monreal G, Nicholson LM, Han B, Joshi MS, Phillips AB, Wold LE, Bauer JA, Gerhardt MA. Cytoskeletal remodeling of desmin is a more accurate measure of cardiac dysfunction than fibrosis or myocyte hypertrophy. Life sciences. 2008;83(23-24):786-794.

8 Krishnamurthy J, Torrice C, Ramsey MR, Kovalev GI, Al-Regaiey K, Su L, Sharpless NE. Ink4a/Arf expression is a biomarker of aging. J Clin Invest. 2004;114(9):1299-1307.

9 Lamy L, Foussat A, Brown EJ, Bornstein P, Ticchioni M, Bernard A. Interactions between CD47 and thrombospondin reduce inflammation. J Immunol. 2007;178(9):5930-5939.

10 Dai DF, Santana LF, Vermulst M, Tomazela DM, Emond MJ, MacCoss MJ, Gollahon K, Martin GM, Loeb LA, Ladiges WC, Rabinovitch PS. Overexpression of catalase targeted to mitochondria attenuates murine cardiac aging. Circulation. 2009;119(21):2789-2797.

11 Agah A, Kyriakides TR, Bornstein P. Proteolysis of cell-surface tissue transglutaminase by matrix metalloproteinase-2 contributes to the adhesive defect and matrix abnormalities in thrombospondin-2-null fibroblasts and mice. Am J Pathol. 2005;167(1):81-88.

12 Vanhoutte D, Schellings MW, Gotte M, Swinnen M, Herias V, Wild MK, Vestweber D, Chorianopoulos E, Cortes V, Rigotti A, Stepp MA, Van de Werf F, Carmeliet P, Pinto YM, Heymans S. Increased expression of syndecan-1 protects against cardiac dilatation and dysfunction after myocardial infarction. Circulation. 2007;115(4):475-482.

13 DeBosch B, Treskov I, Lupu TS, Weinheimer C, Kovacs A, Courtois M, Muslin AJ. Akt1 is required for physiological cardiac growth. Circulation. 2006;113(17):2097-2104.

14 Hannigan GE, Coles JG, Dedhar S. Integrin-linked kinase at the heart of cardiac contractility, repair, and disease. Circ Res. 2007;100(10):1408-1414.

15 Calzada MJ, Annis DS, Zeng B, Marcinkiewicz C, Banas B, Lawler J, Mosher DF, Roberts DD. Identification of novel beta1 integrin binding sites in the type 1 and type 2 repeats of thrombospondin-1. J Biol Chem. 2004;279(40):41734-41743.

16 Manning BD, Cantley LC. AKT/PKB signaling: navigating downstream. Cell. 2007;129(7):1261-1274.

17 Li M, Chiou KR, Bugayenko A, Irani K, Kass DA. Reduced wall compliance suppresses Akt-dependent apoptosis protection stimulated by pulse perfusion. Circ Res. 2005;97(6):587-595.

18 Donnini S, Morbidelli L, Taraboletti G, Ziche M. ERK1-2 and p38 MAPK regulate MMP/TIMP balance and function in response to thrombospondin-1 fragments in the microvascular endothelium. Life sciences. 2004;74(24):2975-2985.

19 Orr AW, Pallero MA, Xiong WC, Murphy-Ullrich JE. Thrombospondin induces RhoA inactivation through FAK-dependent signaling to stimulate focal adhesion disassembly. J Biol Chem. 2004;279(47):4898348992.

20 Fischer P, Hilfiker-Kleiner D. Survival pathways in hypertrophy and heart failure: the gp130-STAT axis. Basic research in cardiology. 2007;102(5):393-411. 
21 DeBosch B, Sambandam N, Weinheimer C, Courtois M, Muslin AJ. Akt2 regulates cardiac metabolism and cardiomyocyte survival. J Biol Chem. 2006;281(43):32841-32851.

22 Kuhn B, del Monte F, Hajjar RJ, Chang YS, Lebeche D, Arab S, Keating MT. Periostin induces proliferation of differentiated cardiomyocytes and promotes cardiac repair. Nat Med. 2007;13(8):962-969.

23 Yoshida Y, Togi K, Matsumae H, Nakashima Y, Kojima Y, Yamamoto H, Ono K, Nakamura T, Kita T, Tanaka M. CCN1 protects cardiac myocytes from oxidative stress via beta1 integrin-Akt pathway. Biochem Biophys Res Commun. 2007;355(3):611-618.

24 Yang Z, Strickland DK, Bornstein P. Extracellular Matrix Metalloproteinase 2 Levels Are Regulated by the Low Density Lipoprotein-related Scavenger Receptor and Thrombospondin 2. J. Biol. Chem. 2001;276(11):8403-8408.

25 Hayashidani S, Tsutsui H, Ikeuchi M, Shiomi T, Matsusaka H, Kubota T, Imanaka-Yoshida K, Itoh T, Takeshita A. Targeted deletion of MMP-2 attenuates early LV rupture and late remodeling after experimental myocardial infarction. Am J Physiol Heart Circ Physiol. 2003;285(3):H1229-1235.

26 Agah A, Kyriakides TR, Letrondo N, Bjorkblom B, Bornstein P. Thrombospondin 2 levels are increased in aged mice: consequences for cutaneous wound healing and angiogenesis. Matrix Biol. 2004;22(7):539547.

27 Bornstein P, Agah A, Kyriakides TR. The role of thrombospondins 1 and 2 in the regulation of cell-matrix interactions, collagen fibril formation, and the response to injury. The international journal of biochemistry \& cell biology. 2004;36(6):1115-1125.

28 Du XJ. Gender modulates cardiac phenotype development in genetically modified mice. Cardiovasc Res. 2004;63(3):510-519.

29 Schellings MW, Vanhoutte D, Swinnen M, Cleutjens JP, Debets J, van Leeuwen RE, d'Hooge J, Van de Werf F, Carmeliet P, Pinto YM, Sage EH, Heymans S. Absence of SPARC results in increased cardiac rupture and dysfunction after acute myocardial infarction. J Exp Med. 2009;206(1):113-123.

30 Heymans S, Luttun A, Nuyens D, Theilmeier G, Creemers E, Moons L, Dyspersin GD, Cleutjens JP, Shipley M, Angellilo A, Levi M, Nube O, Baker A, Keshet E, Lupu F, Herbert JM, Smits JF, Shapiro SD, Baes M, Borgers M, Collen D, Daemen MJ, Carmeliet P. Inhibition of plasminogen activators or matrix metalloproteinases prevents cardiac rupture but impairs therapeutic angiogenesis and causes cardiac failure. Nat Med. Vol 5; 1999:1135-1142.

31 Daya S, Berns KI. Gene therapy using adeno-associated virus vectors. Clinical microbiology reviews. 2008;21(4):583-593. 


\section{Chapter 3}

\section{Supplemental material}

\section{Expanded methods}

\section{AAV9-TSP-2 construction and production}

AAV-CMV-GFP vectors expressed green fluorescent protein (GFP) from the human cytomegalovirus (CMV) promoter and were derived by cloning GFP into pAAV-MCS (Stratagene). AAV-CMV-TSP-2 vectors expressed TSP-2 from the CMV promoter and were derived by cloning TSP-2 into PAAV-MCS (Stratagene). Both vectors contained a b-globin intron downstream of CMV and a human growth hormone polyadenylation signal. 293 cells were cultured in Dulbecco's modified Eagle's medium (DMEM) supplemented with $2 \mathrm{mM}$ L-glutamine (Gln), $100 \mathrm{IU} / \mathrm{ml}$ penicillin, $100 \mu \mathrm{g} / \mathrm{ml}$ streptomycin and $10 \%$ heat-inactivated fetal bovine serum (FBS, Invitrogen, Merelbeke, Belgium). AAV9-CMV-GFP or AAV9-CMV-TSP-2 vectors were produced at high-titer by calcium phosphate transfection of 293 cells with AAV-CMV-GFP or AAV-CMV-TSP-2 vector DNA, an adenoviral helper plasmid and AAV helper plasmids expressing AAV Rep2 and AAV Cap9 for production of AAV9 serotypes, as previously described. ${ }^{1}$ Two days posttransfection, cells were lysed by successive freeze-thaw cycles and sonication. Lysates were treated with benzonase (Merck) and deoxycholate (Sigma-Aldrich) and subsequently subjected to three successive rounds of cesium chloride density ultracentrifugation. The fractions containing the AAV particles were concentrated using an Amicon filter (Millipore) and washed with PBS $1 \mathrm{mM} \mathrm{MgCl2}$. Vector titer was determined in triplicate and repeated twice by quantitative PCR analysis conducted on a ABI 7700 (Applied Biosystems) using Lux primers (Invitrogen) specific for the polyadenylation signal (primer sequences: Forward: TCTATTGGGAACCAAGCTGGAGG Reverse: AGGAGGCGGAGATTGCAGTG), as described previously. The standard consisted of serially diluted AAV-CMV-GFP vector plasmids of known quantity.

\section{Immunohistochemical and electron microscopic analysis}

After the study period, all mice were anaesthetized, and hearts were taken out and prepared for further histological and molecular analysis. Lungs, left (LV) and right ventricles (RV) were dissected, blotted dry and weighed. Immunostainings on paraffin sections were performed using antibodies against p16 (BD bioscience pharmingen, San Diego, CA), Laminin (Sigma), desmin (Abcam, Cambridge, MA), CD45 (BD Bioscience Pharmingen), active cleaved caspase-3 (Upstate Biotechnology, Euromedex, Souffelweyersheim, France), ubiquitin (Chemicon, Temecula, CA), $\alpha$-smooth muscle cell actin 
(Dako, Leuven, Belgium), CD31 (Dako, Leuven, Belgium) and $\varepsilon$-lysyl- $\gamma$-glutaminyl crosslinks (Abcam, Cambridge, MA) as previously described. ${ }^{2-4}$ For TSP-2 (BD Bioscience Pharmingen) sections were subsequently incubated with Biotin labeled secondary antibody followed by amplification with the signal amplification system steptavidinHRP-C_fluorescein (Perkin Elmer). Nuclei were stained with DAPI (invitrogen). ${ }^{3}$ The quantity of collagen was determined as the percentage of Sirius Red staining area per total cardiac area. ${ }^{2-5}$ The quality of the deposited collagen matrix was studied with Sirius Red polarization microscopy as previously described. ${ }^{3,4}$

Morphometric analysis was performed with a Leitz DMRXE microscope (Leica Imaging systems Ltd), a 3CCD color video camera (CXC-93-OP, Sony) and a Leica Qwin software system by persons unaware of the genotype.

For electron microscopic scanning, hearts were treated with $1.5 \%$ glutaraldehyde fixative buffered in $0.067 \mathrm{M}$ cacodylate at $\mathrm{pH}$ 7.4. These hearts were washed in cacodylate buffer and transferred to a $1 \%$ OsO4 fixative solution buffered with phosphatebuffered saline $0.1 \mathrm{M} \mathrm{pH} 7.4$ for subsequent immersion fixation for 1 hour at $4^{\circ} \mathrm{C}$. After

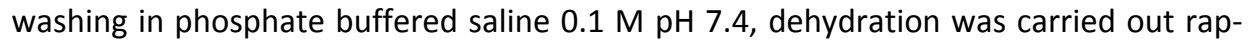
idly in graded ethanol series, followed by embedding in Epon. Hearts were examined at the University of Maastricht (EM unit, Molecular Cell biology) with a Philips CM 100 electron microscope (F.E.I., Eindhoven, The Netherlands) at an accelerating voltage of $80 \mathrm{KV}$.

\section{Zymographic MMP- and tissue transglutaminase activity with aging}

Heart tissues were homogenized and extracted, and protein concentrations were determined. MMP-2 and MMP-9 zymographic activites were determined as described. ${ }^{2}$ The activity of tissue transglutaminase (tTG), a collagen cross-linking enzyme which is inactivated by MMP-2, was measured in homogenized heart tissues by incorporation of biotinylated cadaverine (BTC) into fibronectin as described. ${ }^{4,6}$

\section{RNA isolation and real time polymerase chain reaction}

RNA was extracted from cardiac tissues using an RNeasy Fibrous Tissue Mini Kit (Qiagen $\mathrm{GmbH}$, Hilden, Germany) and was stored at $-80^{\circ} \mathrm{C}$. Transcript levels of TSP-1, TSP2, TSP-3, TSP-4, Superoxide Dismutase-2 (SOD2), Glutathione peroxidase-1 (GPx1) and Catalase1 (CAT1) were determined with a real-time fluorescence detection method as previously described7 (Applied Biosystems), mouse glyceraldehyde 3-phosphate dehydrogenase (GAPDH) was used as an endogenous housekeeping gene. The primers used for this study are presented in supplemental table1.

Transcript levels of TGF- $\beta$, IL-1 $\beta$, IL- 6 and IL-12 were determined using TaqMan low density arrays and were performed according to the manufacturer's instructions. ${ }^{8}$ Each TaqMan gene expression assay contains a forward and reverse primer for each of the 
chosen genes. 18S RNA was incorporated in our customized TaqMan Low-Density Array as internal standard.

\section{Immunoblotting}

Immunoblotting was performed on heart lysates for TSP-1 (kindly provided by Prof. Dr. M. Hoylaerts), TSP-2 (BD), TSP-3 (Santa Cruz biotechnology, Heidelberg, Germany), Integrin Linked kinase (ILK) (Cell signaling Technology Inc., Beverly, MA, USA), Src (Cell singaling), protein kinase A or Akt (Cel signaling) and their phosphorylated forms, and GAPDH (cell signaling) as previously described. ${ }^{3,4}$ Protein levels were expressed relative to protein levels of GAPDH.

\section{ILK activity and active TGF-beta assays}

Immunoprecipitation of ILK in TSP-2 KO and WT heart homogenates together with the kinase activity assay were carried out as detailed in Weaver M. et al. ${ }^{9}$

To determine active TGF- $\beta 1$ protein levels, a TGF- $\beta 1$ assay was performed according to the manufacturer's instructions (R\&D systems, Minneapolis, MN, USA). Cardiac tissue extracts were pre-adjusted to $1.0 \mathrm{mg} / \mathrm{mL}$ in protein. ${ }^{10}$

\section{Echocardiographic measurements}

Transthoracic echocardiographic examination was performed using a $13-\mathrm{mHz}$ transducer (i13L, GE Vingmed, Horten, Norway) on a Vingmed Vivid 7 scanner (GE Vingmed,Horten, Norway) in anesthetized mice (2\% isoflurane). LV diameters at enddiastole (LVEDD) and end-systole (LVESD), septal wall thickness (SW diast), and LV posterior wall in end diastole (PW diast) were measured, and fractional shortening (FS) was calculated as described previously. ${ }^{5,11}$

\section{In vitro experimental approaches}

A rat TSP-2 (rTSP-2) short hairpin RNA (shRNA)-expressing lentiviral vector was generated by annealing complementary shTSP-2 oligonucleotides (Supplemental table 1) and ligating them into HpAI-Xhol-digested pLL3.7 puro vector DNA (modified from a donation by L. van Parijs, Massachusetts Institute of Technology, Cambridge, MA). Lentiviral production was performed as described previously, and viral supernatant was harvested after 48 hours. ${ }^{12}$ Neonatal rat ventricular cardiac myocytes (NRCMs) were isolated by enzymatic disassociation of 1- to 2-days-old neonatal rat hearts.

As neonatal hearts produce a significant amount of TSP-2 -at least- until 6 days after birth ${ }^{13}$, the use of NRCMs was suitable for our experimental setup. NRCMs were infected with lentivirus containing shRNA against TSP-2 or unrelated shRNA lentiviral vector (control; Sigma) and harvested after 10 days as previously described. ${ }^{12}$ 
Mouse monoclonal anti-TSP antibodies, Ab.3 clone C6.7 (indicated as $\alpha$-TSPCD47) and Ab.1 clone A4.1 (indicated as $\alpha$-TSPCD36) (Labvision Mouse, Duiven, the Netherlands), were used to block respectively the CD47 binding site ( $C$ terminal domain) and the CD36 binding site respectively on TSP-1 and TSP- $2 .{ }^{14}$ Cell lysates were prepared for further analysis of protein expression and transcript levels.

Supplemental table 1. List of primers for Real-Time PCR and for shTSP-2 production

\begin{tabular}{|c|c|c|}
\hline Gene & Primer & Sequence $5^{\prime}-3^{\prime}$ \\
\hline \multirow[t]{3}{*}{ Mouse GAPDH ${ }^{a}$} & $\mathrm{~F}$ & ACGTGCCGCCTGGAGA \\
\hline & $\mathrm{R}$ & CCCTCAGATGCCTGCTTCA \\
\hline & probe & CACCTTCTTGATGTCATCATACTTGGCAGG \\
\hline \multirow[t]{3}{*}{ Mouse-CAT1 } & $\mathrm{F}$ & CCTGGCATCGGAGCCCA \\
\hline & $\mathrm{R}$ & GTGTCCGGGTAGGCAAAAAG \\
\hline & Probe & CCCTGACAAAATGCTTCAGGGCCG \\
\hline \multirow[t]{3}{*}{ Mouse-Gxp } & $\mathrm{F}$ & GACTGGTGGTGCTCGGTTTC \\
\hline & $\mathrm{R}$ & CAGAATCTCTTCATTCTTGCCAT \\
\hline & Probe & CGTGCAATCAGTTCGGACACCAGG \\
\hline \multirow[t]{2}{*}{ Rat-shTSP-2 $2^{b}$} & Sense & GATATCTGCTTCTCAATTATTCAAGAGATAATTGAGAAGCAGATATCTTTTTTC \\
\hline & Antisense & TCGAGAAAAAGATATCTGCTTCTCAATTATCTCTTGAATAATTGAGAAGCAGATATC \\
\hline
\end{tabular}

${ }^{a}$ Housekeeping gene: GAPDH,glyceraldehydes3-phhosphate dehydrogenase

${ }^{b}$ rat-shTSP-2 oligonucleotides, hairpin structure in bold.

Mouse TSP-1 ID: Mm 01335419_g1; Mouse TSP-2 ID: Mm01279241_m1;

Mouse TSP-3 ID: Mm 01212335_m1; Mouse TSP-4 ID: Mm 03003598_s1

Mouse SOD2 ID: Mm 00449726_m1

\section{Force measurements in single permeabilized cardiomyocytes}

Force measurements were performed in single, mechanically isolated cardiomyocytes as described previously. ${ }^{15,16}$ Cardiac samples were defrosted in relaxing solution (free Mg 1, KCl 100, EGTA 2, Mg-ATP 4 and imidazole $10 \mathrm{mmol} / \mathrm{L} ; \mathrm{pH} 7.0$ ), mechanically disrupted and incubated for 5 minutes in relaxing solution supplemented with $0.5 \%$ Triton X-100 to remove all membrane structures. Subsequently, cells were washed twice in relaxing solution, after which single cardiomyocytes were attached with silicone adhesive between a force transducer and a motor. Sarcomere length of isolated cardiomyocytes was adjusted to $2.2 \mu \mathrm{m}$ and myocytes were subjected to both relaxing and activating solutions. Their PCA $(-10 \log [\mathrm{Ca} 2+])$ ranged from 9.0 (relaxing) to 4.5 (maximal activation), which was used to calculate maximal calcium-activated isometric force. All force values were normalized for cardiomyocyte cross-sectional area. Exposure to a series of solutions with intermediate PCA yielded the baseline force-PCA relation. On transfer of the myocyte from relaxing to activating solution, isometric force started to develop. Once a steady force level was reached, the cell was shortened within $1 \mathrm{~ms}$ to $80 \%$ of its original length (slack test) to determine the baseline of the force transducer. The distance between the baseline and the steady force level is the total force (Ftotal). After 20 ms, the cell was restretched and returned to the relax- 
ing solution, in which a second slack test of 10 -seconds' duration was performed to determine passive passive force. The difference between total force and passive force equalled active force. Ca2+-sensitivity of the contractile apparatus (pCa50) corresponded to the PCA, at which active force reached $50 \%$ of the value observed at maximal activation. After measurement of the baseline force-PCA relation, myocytes were incubated for 40 minutes in relaxing solution supplemented with the catalytic subunit of protein kinase A (PKA, $100 \mathrm{U} / \mathrm{mL}$; Sigma, batch-12K7495) and $6 \mathrm{mmol} / \mathrm{L}$ dithiothreitol (DTT, MP-Biochemicals).

\section{Phosphorylation status of myofilament proteins}

Myofilament protein phosphorylation was determined using Pro-Q Diamond Phosphoprotein Stain as described previously. ${ }^{17}$ To preserve the endogenous phosphorylation status, frozen samples were homogenized in liquid nitrogen and re-suspended in $1 \mathrm{~mL}$ cold $10 \%$ trichloroacetic acid solution (TCA; dissolved in acetone containing $0.1 \%(\mathrm{w} / \mathrm{v})$ DTT). TCA-treated tissue pellets were homogenized in sample buffer containing $15 \%$ glycerol, $62.5 \mathrm{mM}$ Tris (pH 6.8), 1\% (w/v) SDS and 2\% (w/v) DTT. Tissue samples were separated on gradient gels (Criterion tris- $\mathrm{HCl} 4-15 \%$ gel, BioRad) and proteins were stained for one hour with Pro-Q Diamond Phosphoprotein Stain. Fixation, washing and de-staining were performed according to the manufacturer's guidelines (Molecular Probes). To assess protein content subsequently gels were stained overnight with SYPRO Ruby stain (Molecular Probes). Phosphorylation status of myofilament proteins was expressed relative to protein expression of cardiac myosin-binding protein $\mathrm{C}$ (MyBP-C) to correct for differences in sample loading. Staining was visualized using the LAS-3000 Image Reader and signals were analyzed with AIDA. 


\section{Supplemental figures}

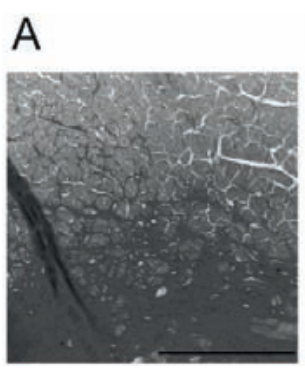

B
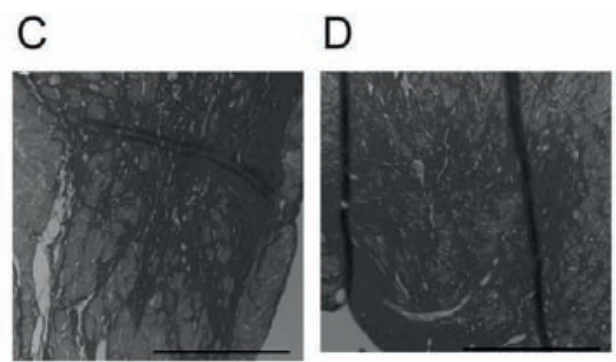

Supplemental figure 1. The loss of TSP-2 resulted in increased cardiac fibrosis. (A-D) Post mortem analysis of Sirius red-stained older male TSP-2 KO hearts revealing increased cardiac fibrosis and cardiomyocyte dropout. A through D represent 4 individual hearts. Bars $=100 \mu \mathrm{m}$.
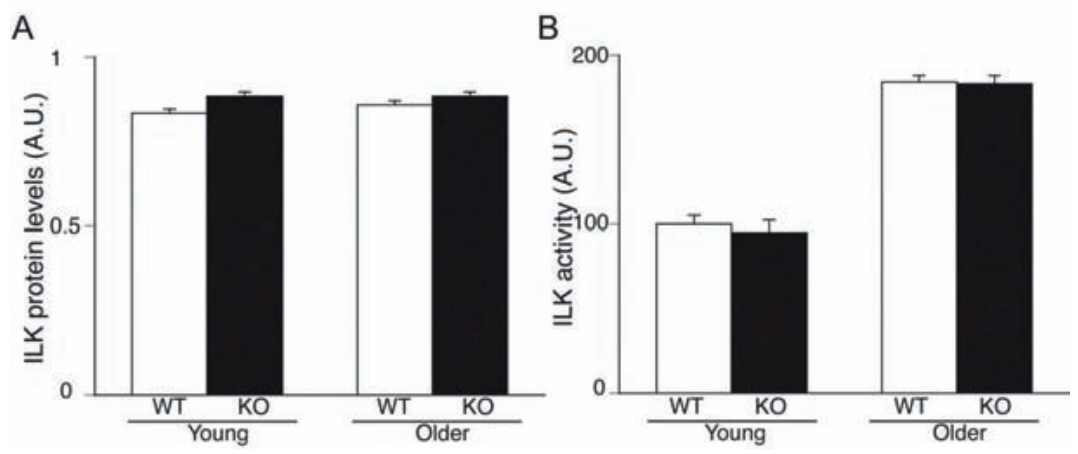

Supplemental figure 2. The loss of TSP-2 did not resulted in differences in ILK activity levels with aging. (A) Immunoblotting for ILK protein levels did not show differences in young and older TSP-2 KO hearts versus age-matched WT littermates ( $n=5$ per genotype per age-group; $P=N S$ ). (B). ILK activity assay showed no significant differences in ILK activity levels between TSP-2 KO and WT hearts in young or older mice. 


\section{Supplemental references}

1 Vandendriessche T, Thorrez L, Acosta-Sanchez A, Petrus I, Wang L, Ma L, L DEW, Iwasaki Y, Gillijns V, Wilson JM, Collen D, Chuah MK. Efficacy and safety of adeno-associated viral vectors based on serotype 8 and 9 vs. lentiviral vectors for hemophilia B gene therapy. J Thromb Haemost. 2007;5(1):16-24.

2 Heymans S, Luttun A, Nuyens D, Theilmeier G, Creemers E, Moons L, Dyspersin GD, Cleutjens JP, Shipley M, Angellilo A, Levi M, Nube O, Baker A, Keshet E, Lupu F, Herbert JM, Smits JF, Shapiro SD, Baes M, Borgers M, Collen D, Daemen MJ, Carmeliet P. Inhibition of plasminogen activators or matrix metalloproteinases prevents cardiac rupture but impairs therapeutic angiogenesis and causes cardiac failure. Nat Med. Vol 5;1999:1135-1142.

3 Schellings MW, Vanhoutte D, Swinnen M, Cleutjens JP, Debets J, van Leeuwen RE, d'Hooge J, Van de Werf F, Carmeliet P, Pinto YM, Sage EH, Heymans S. Absence of SPARC results in increased cardiac rupture and dysfunction after acute myocardial infarction. J Exp Med. 2009;206(1):113-123.

4 Vanhoutte D, Schellings MW, Gotte M, Swinnen M, Herias V, Wild MK, Vestweber D, Chorianopoulos E, Cortes V, Rigotti A, Stepp MA, Van de Werf F, Carmeliet P, Pinto YM, Heymans S. Increased expression of syndecan-1 protects against cardiac dilatation and dysfunction after myocardial infarction. Circulation. 2007;115(4):475-482.

5 Heymans S, Lupu F, Terclavers S, Vanwetswinkel B, Herbert JM, Baker A, Collen D, Carmeliet P, Moons L. Loss or Inhibition of UPA or MMP-9 Attenuates LV Remodeling and Dysfunction after Acute Pressure Overload in Mice. Am J Pathol. 2005;166(1):15-25.

6 Agah A, Kyriakides TR, Bornstein P. Proteolysis of cell-surface tissue transglutaminase by matrix metalloproteinase-2 contributes to the adhesive defect and matrix abnormalities in thrombospondin-2-null fibroblasts and mice. Am J Pathol. 2005;167(1):81-88.

7 Dai DF, Santana LF, Vermulst M, Tomazela DM, Emond MJ, MacCoss MJ, Gollahon K, Martin GM, Loeb LA, Ladiges WC, Rabinovitch PS. Overexpression of catalase targeted to mitochondria attenuates murine cardiac aging. Circulation. 2009;119(21):2789-2797.

8 Abruzzo LV, Lee KY, Fuller A, Silverman A, Keating MJ, Medeiros LJ, Coombes KR. Validation of oligonucleotide microarray data using microfluidic low-density arrays: a new statistical method to normalize real-time RT-PCR data. BioTechniques. 2005;38(5):785-792.

9 Weaver MS, Workman G, Sage EH. The copper binding domain of SPARC mediates cell survival in vitro via interaction with integrin beta1 and activation of integrin-linked kinase. J Biol Chem. 2008;283(33):22826-22837.

10 Mo W, Brecklin C, Garber SL, Song RH, Pegoraro AA, Au J, Arruda JA, Dunea G, Singh AK. Changes in collagenases and TGF-beta precede structural alterations in a model of chronic renal fibrosis. Kidney international. 1999;56(1):145-153.

11 Schroen B, Heymans S, Sharma U, Blankesteijn WM, Pokharel S, Cleutjens JP, Porter JG, Evelo CT, Duisters R, van Leeuwen RE, Janssen BJ, Debets JJ, Smits JF, Daemen MJ, Crijns HJ, Bornstein P, Pinto YM. Thrombospondin-2 is essential for myocardial matrix integrity: increased expression identifies failureprone cardiac hypertrophy. Circ Res. 2004;95(5):515-522.

12 Schroen B, Leenders JJ, van Erk A, Bertrand AT, van Loon M, van Leeuwen RE, Kubben N, Duisters RF, Schellings MW, Janssen BJ, Debets JJ, Schwake M, Hoydal MA, Heymans S, Saftig P, Pinto YM. Lysosomal integral membrane protein 2 is a novel component of the cardiac intercalated disc and vital for loadinduced cardiac myocyte hypertrophy. J Exp Med. 2007;204(5):1227-1235.

13 Iruela-Arispe ML, Liska DJ, Sage EH, Bornstein P. Differential expression of thrombospondin 1, 2, and 3 during murine development. Dev Dyn. 1993;197(1):40-56.

14 Calzada MJ, Annis DS, Zeng B, Marcinkiewicz C, Banas B, Lawler J, Mosher DF, Roberts DD. Identification of novel beta1 integrin binding sites in the type 1 and type 2 repeats of thrombospondin-1.J Biol Chem. 2004;279(40):41734-41743.

15 van der Velden J, Papp Z, Boontje NM, Zaremba R, de Jong JW, Janssen PM, Hasenfuss G, Stienen GJ. The effect of myosin light chain 2 dephosphorylation on $\mathrm{Ca} 2+$-sensitivity of force is enhanced in failing human hearts. Cardiovasc Res. 2003;57(2):505-514. 
16 van der Velden J, Papp Z, Zaremba R, Boontje NM, de Jong JW, Owen VJ, Burton PB, Goldmann P, Jaquet $\mathrm{K}$, Stienen GJ. Increased Ca2+-sensitivity of the contractile apparatus in end-stage human heart failure results from altered phosphorylation of contractile proteins. Cardiovasc Res. 2003;57(1):37-47.

17 Zaremba R, Merkus D, Hamdani N, Lamers J, Paulus W, C. dR, Duncker D, Stienen G, van der Velden J. Quantitative analysis of myofilament protein phosphorylation in small cardiac biopsies. Proteomics Clinical Application. 2007;1(10):1285-1290. 


\section{Chapter 4}

\section{Absence of thrombospondin-2 increases cardiomyocyte damage and matrix disruption in doxorubicin-induced cardiomyopathy}

Geert C. van Almen*, Melissa Swinnen*, Paolo Carai, Wouter Verhesen, Jack P.M. Cleutjens, Jan D’hooge, Fons K. Verheyen, Yigal M. Pinto, Blanche Schroen, Peter Carmeliet, Stephane Heymans

*These authors contributed equally to this manuscript

Modified from Journal of Molecular and Cellular Cardiology. 2011 May;51:318-328 


\begin{abstract}
Clinical use of the antineoplastic agent doxorubicin (DOX) is limited by its cardiomyocyte toxicity. Attempts to decrease cardiomyocyte injury showed promising results in vitro, but failed to reduce the adverse effects of DOX in vivo, suggesting that other mechanisms contribute to its cardiotoxicity as well. Evidence that DOX also induces cardiac injury by compromising extracellular matrix integrity is lacking. The matricellular protein thrombospondin-2 (TSP-2) is known for its matrix-preserving function, and for modulating cellular function. Here, we investigated whether TSP-2 modulates the process of doxorubicin-induced cardiomyopathy (DOX-CMP). TSP-2-knockout (TSP2-KO) and wild-type (WT) mice were treated with DOX (2mg/kg/week) for 12 weeks to induce DOX-CMP. Mortality was significantly increased in TSP-2-KO compared to WT mice. Surviving DOX-treated TSP-2-KO mice had depressed cardiac function compared to WT animals, accompanied by increased cardiomyocyte apoptosis and matrix damage. Enhanced myocyte damage in the absence of TSP- 2 was associated with impaired activation of the Akt signaling pathway in TSP-2-KO compared to WT. The absence of TSP-2, in vivo and in vitro, reduced Akt activation both under non-treated conditions and after DOX. Importantly, inhibition of Akt phosphorylation in cardiomyocytes significantly reduced TSP-2 expression, unveiling a unique feedback loop between Akt and TSP-2. Finally, enhanced matrix disruption in DOX-treated TSP-2-KO hearts went along with increased matrix metalloproteinase-2 levels. Taken together, this study is the first to provide evidence for the implication of the matrix element TSP-2 in protecting against DOX-induced cardiac injury and dysfunction.
\end{abstract}




\section{Introduction}

Doxorubicin (DOX) is an anticancer drug commonly used for the treatment of hematologic malignancies and solid tumors. Its clinical application is limited by a doserelated cardiotoxicity, which is characterized by progressive cardiac dilatation, contractile dysfunction and ultimately heart failure. ${ }^{1}$ Formation of reactive oxygen species (ROS) and resulting cardiomyocyte damage is generally believed to be the primary cause of its cardiotoxicity. ${ }^{2}$ Interestingly, interventions that aim to decrease oxidative stress by treatment with exogenous antioxidants or overexpression of endogenous antioxidant enzymes showed promising results in vitro and in vivo ${ }^{3-6}$, but failed to reduce the incidence of cardiotoxicity in patients treated with DOX. This suggests that mechanisms other than anti-oxidants contribute to the response to DOX as well.

DOX-induced cardiomyopathy (DOX-CMP) has been associated with loss of myocardial interstitial collagen and enhanced expression of non-structural glycoproteins in the matrix ${ }^{7-11}$, but clear evidence on the implication of specific matrix components in DOX-cardiotoxicity is completely lacking. By recognizing the possible duality of DOXcardiotoxicity, we hypothesize that matrix components mediate the development of DOX-CMP. Here, we address whether the matricellular protein thrombospondin-2 (TSP-2) affects DOX-induced cardiotoxic remodeling by mediating matrix alterations or cardiomyocyte toxicity.

Thrombospondins (TSPs) are secreted glycoproteins within the ECM that belong to the group of matricellular proteins, and act as key players in the cross-talk between the matrix and surrounding cells. TSP-2 is known to control matrix metalloproteinase (MMP) activity, whereas its binding to cell surface receptors regulates cellular function during wound healing and tumor formation by linking the matrix to intracellular path-

ways. ${ }^{12-14}$ In the heart, TSP-2 was initially considered to be essential for strengthening the cardiac matrix in hypertensive heart disease. ${ }^{15}$ Recent evidence, supports an additional role for TSP-2 as it was shown to have a pro-survival effect on cardiomyocytes during physiological aging of the heart. ${ }^{16}$ Here, we show that the pro-survival and matrix-preserving actions of TSP-2 protect against cardiomyocyte loss and ECM weakening that contribute to DOX-induced heart failure.

\section{Methods}

An expanded methods section is provided in the Supplemental material (pag. 92).

\section{TSP-2-KO mice and experimental approaches}

The present study conforms with the Guide for the care and use of laboratory animals published by the US National Institutes of Health (NIH Publication NO. 85-23, revised 1996) and was approved by the Institutional Animal Research Committee according to 
the rules formulated in the Dutch and Belgian law on care and use of experimental animals.

DOX-CMP was induced in 10-12 weeks old male and female TSP-2-KO mice (male, $n=24$; female, $n=26$ ) and their wild-type (WT) littermates (male, $n=17$; female, $n=15$ ) on a C57BL6/129SvJ/EMS+Ter genetic background. ${ }^{17}$ Sham TSP-2-KO (male, $n=11$; female, $n=9$ ) and WT (male, $n=14$; female, $n=10$ ) mice were treated with saline. A clinically relevant dose of $24 \mathrm{mg} / \mathrm{kg}$ DOX (Pfizer, \#32001013) was administered by a weekly intra-peritoneal injection of $2 \mathrm{mg} / \mathrm{kg}$ for 12 weeks. Cardiac function was assessed by echocardiographic examination at baseline and after 12 weeks of DOX treatment. Subsequently, hearts were taken out and prepared for histological and molecular analyses.

\section{In vitro experimental approaches}

Primary neonatal rat cardiomyocytes (NRCMs) and cardiac fibroblasts (NRCFs) were isolated from the hearts of 1- to 3-day-old Lewis rats (Janvier, Le Genest Saint Isle, France). TSP-2 expression in NRCMs and NRCFs was repressed by transfection with short-interference RNA against rat-TSP-2 (siTSP-2) (Dharmacon, \#L-081165-01) followed by exposure to $1 \mu \mathrm{M}$ DOX (Pfizer, \#32001013) for 24 hours. To study the effect of Akt signaling on the expression of TSP-2, NRCMs were cultured for 24 hours in the presence of $10 \mu \mathrm{M}$ Akt-inhibitor VIII (Calbiochem, \#124017).

\section{Histopathological and molecular analysis}

Cardiac tissue damage and ultrastructural morphology after DOX treatment were examined on hematoxylin and eosin stained tissue sections and via electron microscopic analysis, respectively. Collagen content was morphometrically determined as percentage sirius red staining per total cardiac tissue area. Cardiomyocyte disarray and apoptosis were measured with immunostaining for desmin (Abcam, Cambridge, UK), active cleaved caspase-3 (Cell Signaling Technology) and TUNEL (Chemicon). Inflammatory infiltration was determined by CD45 (BD Bioscience Pharmingen) immunostaining. The presence of peroxynitrite, a marker of oxidative stress, was assessed by immunostaining for 3-nitrotyrosine (Upstate). Active cleaved caspase-3 and TUNEL staining were presented as the percentage positive staining cardiomyocyte nuclei per total cardiomyocyte nuclei. Cardiomyocyte density and cardiomyocyte area were determined and used to calculate the cardiomyocyte content of the left ventricle.

Transcript levels of MMP-2 and -9, TIMP-1, $-2,-3$, and -4 , catalase-1 (CAT), superoxide dismutase-2 (SOD2) [ID: Mm00449726_m1], glutathione peroxidase 1 (GPX1), and Rac1 were determined relative to glyceraldehyde-3-phosphate dehydrogenase (GAPDH) expression, using the Applied Biosystems 7500 Fast Real-Time PCR System. Primer sequences are listed in Supplemental table 1. 
To determine the extent of Akt activation and TSP-2 protein expression in vivo in left ventricular heart tissue and in vitro in cultured NRCMs, immunoblotting was performed to detect TSP-2 (BD Transduction Laboratories), phospho-Akt (Ser473) (Cell Signaling Technology), total-Akt (Cell Signaling Technology), and GAPDH (Fitzgerald). Transcriptional repression by siTSP-2 in NRCMs and NRCFs was measured using RealTime PCR. Primer sequences are listed in Supplemental table 1.

Zymography, based on a SDS-PAGE method, was performed to determine zymographic levels of MMP-2 and MMP-9 in homogenized heart tissue of DOX- and control-treated WT and TSP-2-KO mice and in vitro in the cell lysates of siTSP-2 or scrambled siRNA transfected NRCMs and NRCFs after DOX exposure.

All data are presented as mean \pm standard error of mean (SEM). Mann-Whitney Utest or unpaired $t$ test were used, when appropriate, to assess statistical significance between groups. Survival curves were obtained by the Kaplan-Meier method, and compared by the Fisher's Exact test. Probability values $<0.05$ were considered statistically significant.

\section{Results}

Increased susceptibility to DOX-CMP in TSP-2-KO mice

No mortality was observed in all sham-treated groups, except for the TSP-2-KO males where two mice died (18 percent, 2 out of 11) (Figure 1A). Chronic DOX-exposure significantly induced mortality in male WT mice (29 percent, 5 out of 17 ; ), but not in WT females (7 percent, 1 out of 15) compared to sham-treated mice (Figure 1A). DOXassociated mortality further increased in the absence of TSP-2 and was significantly higher in male (67 percent, 16 out of 24) and female (38 percent, 10 out of 26) TSP-2KO mice compared to gender-matched WT animals (Figure $1 \mathrm{~A}$ ). Interestingly, male TSP-2-KO mice seemed more prone to the cardiotoxic effects of DOX, as mortality already occurred in the second week of exposure, at a cumulative dose of $4 \mathrm{mg} / \mathrm{kg}$ of DOX, whereas no mortality was observed until eight weeks of treatment in females, which causes the overall mortality in males to be increased compared to females $(P=0.05)$ (Figure $1 \mathrm{~A})$. Post-mortem analysis revealed large numbers of erythrocytes residing within the myocardial tissue of male TSP-2-KO mice, suggesting severe matrix weakening that causes intra-myocardial and pericardial bleeding (Figure 1B and C). 


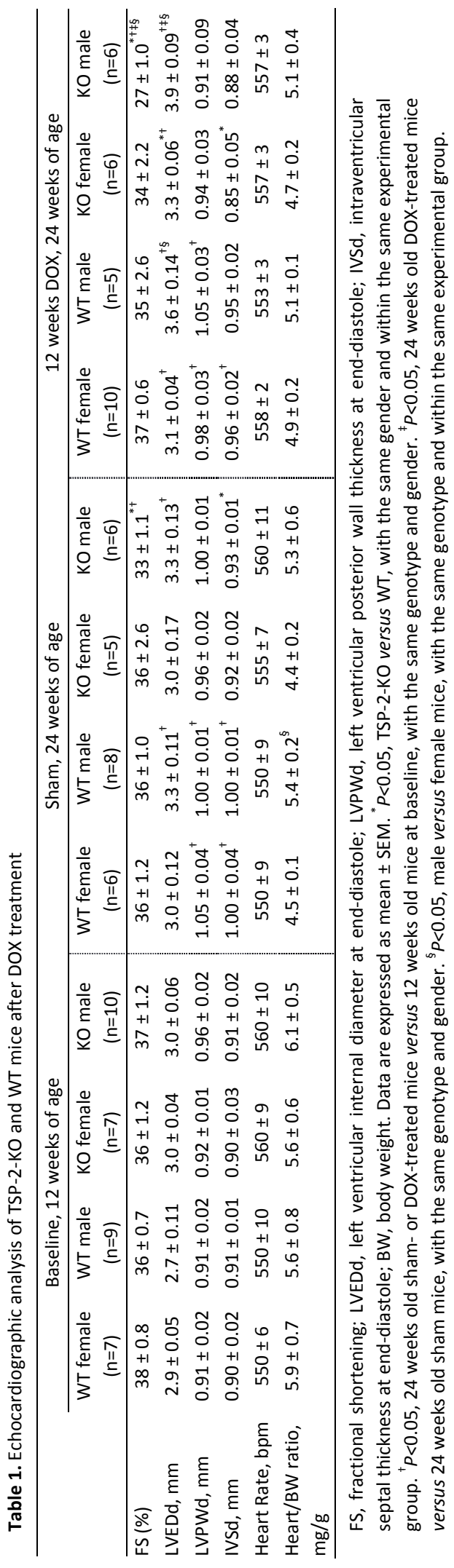


A

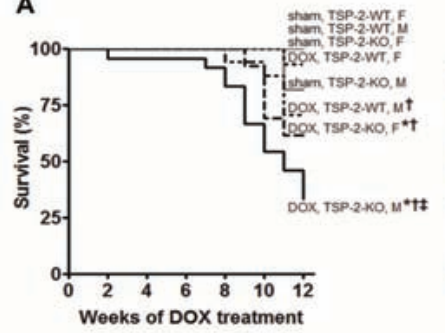

TSP-2-KO, DOX (post-mortem)
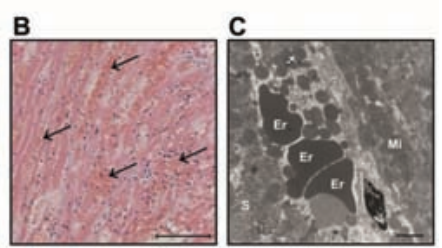

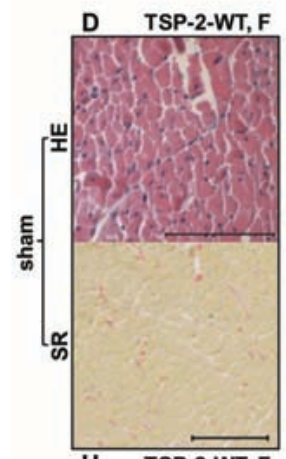

E TSP-2-WT, $M$
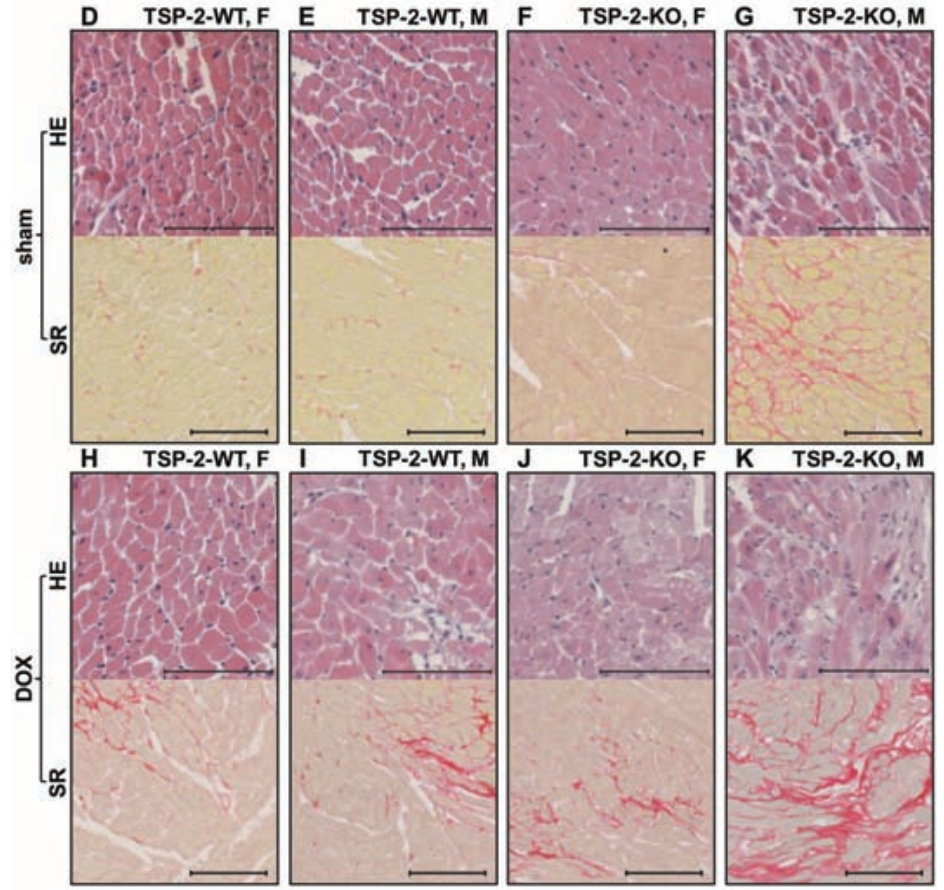

Figure 1. Increased mortality, cardiomyocyte damage and scar forming fibrosis in DOX-treated TSP-2-KO mice. (A) Survival curve of DOX-treated TSP-2-KO and WT mice. DOX significantly induced mortality in WT male and TSP-2-KO mice compared to sham-treatment, and was most pronounced in male TSP-2-KO mice. $M$, indicates male; $F$, indicates female. ${ }^{*} P<0.05$, TSP-2-KO versus WT mice with the same gender. $+P<0.05$, DOX-treated mice versus sham-treated mice. $¥ P=0.05$, male versus female mice, with the same genotype and within the same experimental group. (B and C) Post-mortem histological and ultrastructural analysis of the myocardial tissue of male TSP-2-KO mice after DOX. (B) Hematoxylin and eosin staining showing intramyocardial bleeding, demonstrated by erythrocytes that reside within the interstitial space. Erythrocytes are indicated by the black arrows. Scale bar represent $100 \mu \mathrm{m}$. (C) Ultrastructural examination confirmed intra-myocardial bleeding in the hearts of male TSP-2-KO mice. Er indicates the infiltrating erythrocytes in the interstitial space. Mi, mitochondrion; S, sarcomere. Scale bar represent $2 \mu \mathrm{m}$. Histological examination showed cardiac tissue damage with scar forming fibrosis after DOX exposure in surviving WT and TSP-2-KO mice. (D-K, upper part) DOX-induced cardiac injury was most pronounced in male TSP-2-deficient mice, as indicated by hematoxylin and eosin staining (HE). (D-K, lower part) Increased tissue damage after DOX was accompanied by enhanced collagen deposition as determined by sirius red staining (SR), and was most pronounced in male TSP-2-KO mice. No significant cardiac damage and fibrosis were noted in WT and TSP2-KO mice at baseline. See supplemental figure 1. Scale bars represent $100 \mu \mathrm{m}$. 
Echocardiographic analysis showed a compromised cardiac function in the absence of TSP-2. Importantly, male TSP-2-KO mice had the most pronounced DOX-associated cardiac dysfunction, characterized by enlargement of the LV cavity and reduced wall thickness (Table 1). In DOX-treated WT and female TSP-2-KO mice, indices of LV dilatation also indicated a tendency towards increased LV dimensions, however they did not reach significance and did not result in reduced fractional shortening (Table 1). Cardiac function and dimensions did not differ between groups at baseline, except for male TSP-2-KO mice who had significantly reduced fractional shortening at 24 weeks of age, in line with our previous study showing a decline of cardiac function with aging (Table 1). ${ }^{16}$ Nevertheless, the additional detrimental effect of DOX was illustrated by further thinning of the LV walls and significantly increased cardiac dilatation in treated TSP-2KO males.

\section{DOX-associated injury increased fibrosis and loss of cardiomyocytes in TSP-2-KO mice}

TSP-2 is implicated in cell-matrix interactions, thereby regulating cellular function and matrix integrity. Therefore, morphological and histological features of cardiomyocytes and the extracellular matrix were examined. DOX-induced cardiac damage was characterized by a progressive loss of cardiomyocytes and increased scar forming fibrosis (Figure 1D-K). No histological abnormalities were observed in mice at baseline (Supplemental figure 1), and cardiac injury was absent in sham-treated WT and female TSP2-KO mice. However, male TSP-2-KO mice showed mild tissue damage with increased fibrosis (Figure 1G, Table 2). Chronic DOX-exposure resulted in cardiac tissue injury in both WT and TSP-2-KO hearts, but was most pronounced in male KO mice (Figure $1 \mathrm{H}-$ $\mathrm{K})$. Electron microscopy substantiated cardiomyocyte specific suffering after DOX in the absence of TSP-2 by showing signs of severe oxidative stress, such as mitochondrial swelling and extraction of mitochondrial matrix and loss and damage of myofilaments and sarcomere structures, which was more pronounced in TSP-2-KO as compared to WT hearts (Supplemental figure 2A-D). Histological examination confirmed the increased myocyte stress after DOX, showing clear desmin disorganization after DOX, in particular when TSP-2 was absent (Figure 2A, Table 2). As expected, enhanced myocyte stress after DOX caused significant induction of cardiomyocyte apoptosis in DOXtreated mice. In line with the profound desmin disorganisazation in TSP-2-KO mice, apoptosis was significantly more induced in these hearts, as demonstrated by cleaved caspase-3 and TUNEL immunostaining (Figure 2B, Table 2). These findings were reflected in a significant decrease in cardiomyocyte density after DOX (Table 2) in TSP-2KO male mice. Because DOX did not cause any differences in cardiomyocyte size (Table 2 ), the overall loss of cardiomyocytes after DOX resulted in a significant decrease in relative myocyte content in the hearts of TSP-2-KO males, as calculated by the ratio of cardiomyocyte density and cross-sectional area (Table 2). The dropout of cardiomyocytes was accompanied by increased collagen deposition after DOX in male TSP-2 KO mice (Figure 1D-K, Table 2). 


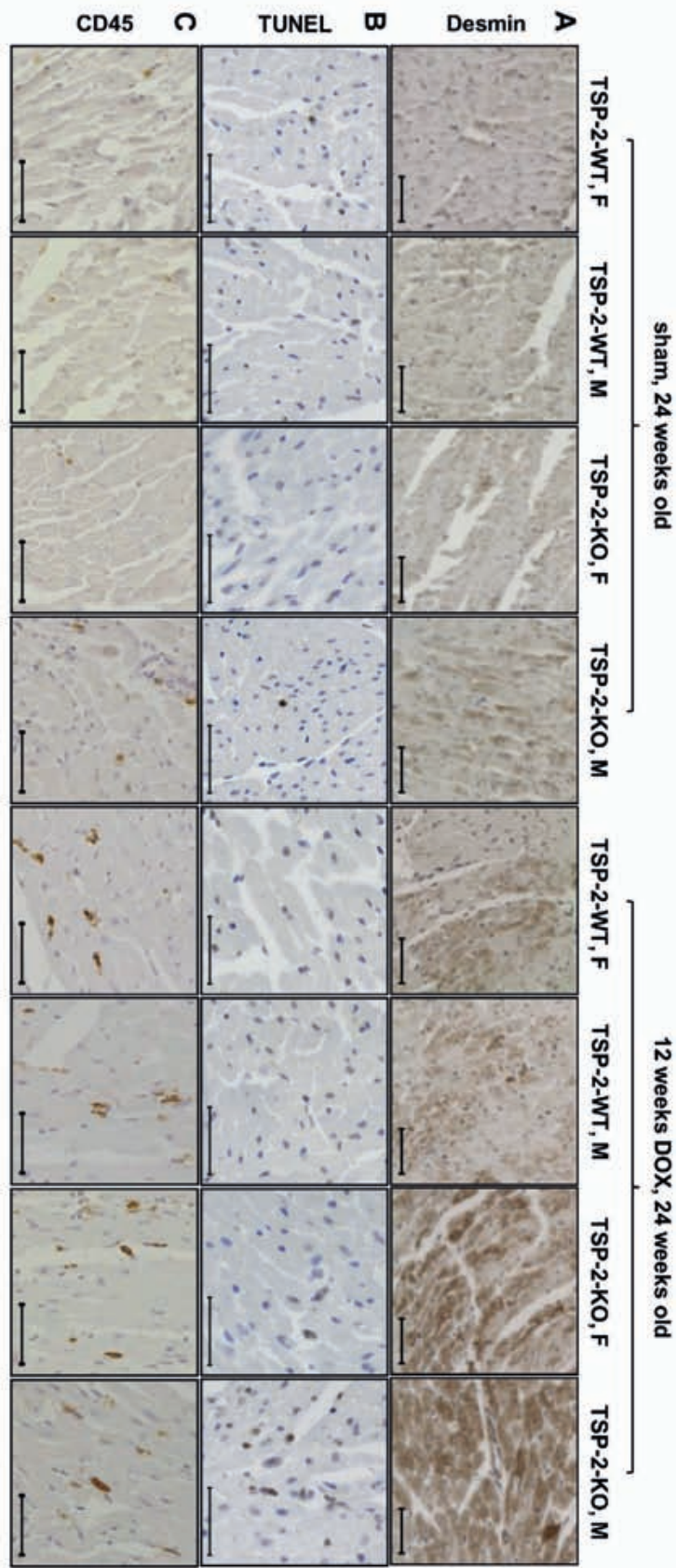

Figure 2. Increased myocyte stress and apoptosis in TSP-2-deficient mice after DOX. Immunohistochemical analysis of DOX- and sham-treated WT and TSP-2-KO mice. (A) Desmin staining showing increased myocyte stress in TSP-2-KO mice. (B) TUNEL staining revealed increased cardiomyocyte apoptosis after DOX, in particular in male mice lacking TSP-2. (C) CD45 was equally induced by DOX in WT and TSP-2-KO mice. Scale bars represent $50 \mu \mathrm{m}$. 


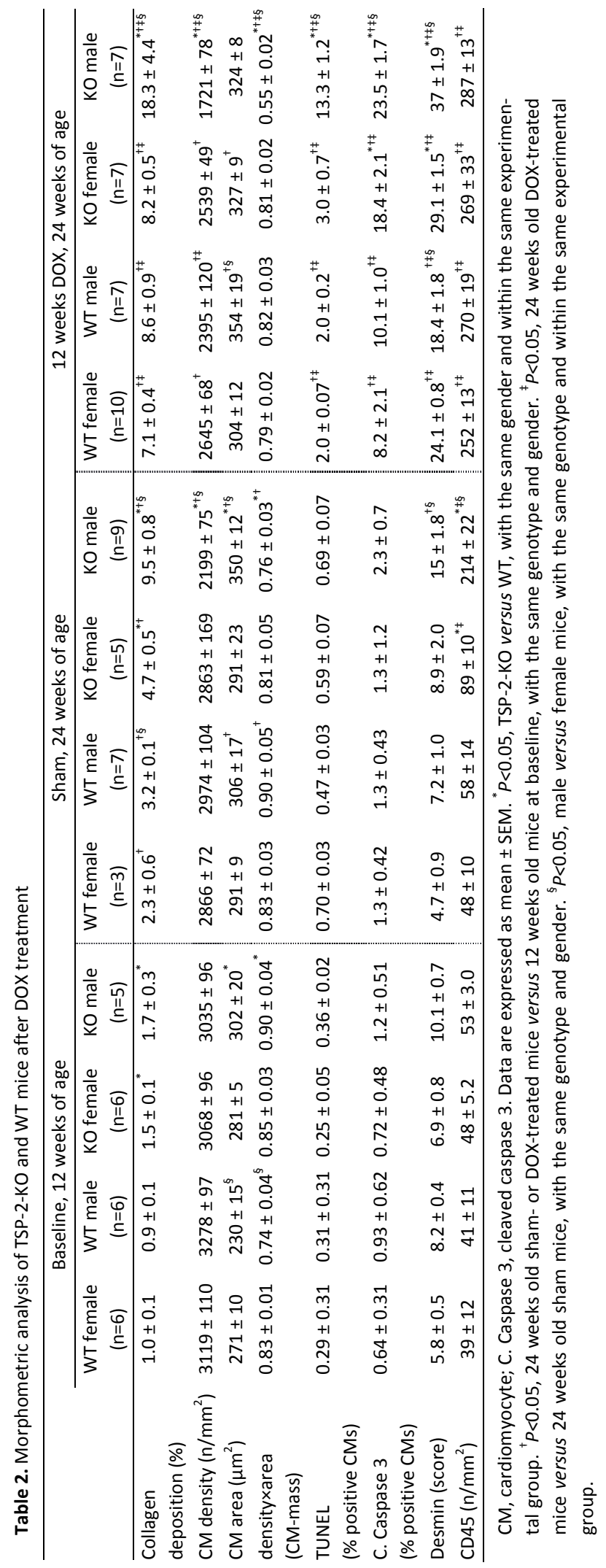


In order to study to what extent inflammation contributes to the phenotype observed in TSP-2-KO mice after DOX, we determined the number of CD45 positive cells in these hearts. No differences were observed at baseline and in the non-treated, sham condition (Figure 2C, Table 2), except for the increase in the twenty-four weeks aged male TSP-2-KO mice as previously described (Figure 2C, Table 2). ${ }^{16}$ DOX significantly enhanced the number CD45-staining leukocytes in both WT and TSP-2-KO hearts (Figure 2C, Table 2). Importantly, CD45 inflammatory cells were induced to an equal extent in WT and TSP-2-deficient hearts of both females and males, indicating that increased DOX-cardiotoxicity in mice lacking TSP-2 is independent of the extent of inflammation.

Together, these data show that the absence of TSP-2 results in increased DOXinduced myocyte stress and apoptosis, followed by enhanced reparative fibrosis.

Oxidative stress and antioxidant mechanisms are equally induced by DOX in TSP-2-KO and WT mice

Increased oxidative stress plays a crucial role in cardiac damage during DOX treatment. ${ }^{18}$ As signs of oxidative damage were more pronounced in the absence of TSP-2, we examined if the genotype affected the extent of oxidative stress or the induction of antioxidant mechanisms. DOX significantly increased 3-nitrotyrosine staining, a marker of oxidative stress-induced peroxynitrite $\left(\mathrm{ONOO}^{-}\right)^{5}$, in both TSP-2-KO and WT hearts (Figure 3A-D), but did not reveal significant differences between both genotypes (Figure $3 \mathrm{E})$. DOX also increased transcript levels of the antioxidants catalase-1 (CAT) and superoxide dismutase-2 (SOD2), but to a similar extent in both WT and TSP-2-KO hearts (Figure 3F and G). Together, these data indicate that increased cardiac injury after DOX in the absence of TSP-2 is not primarily caused by differences in oxidative stress or differently induced antioxidant mechanisms. 


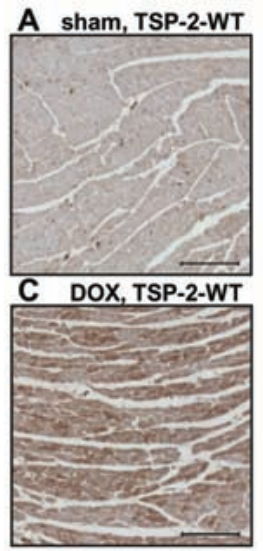

$\mathbf{F}$

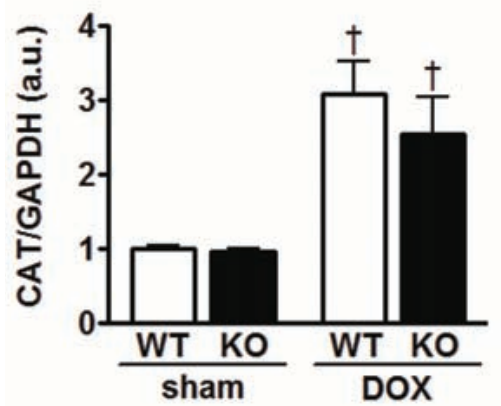

E

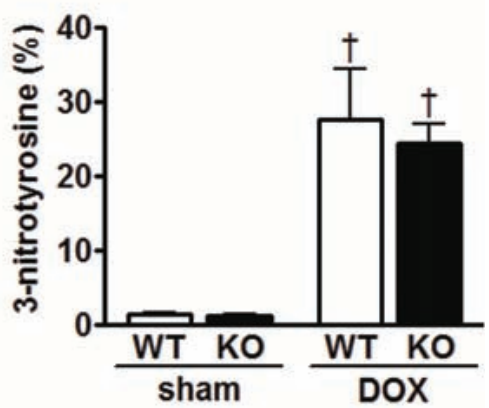

G

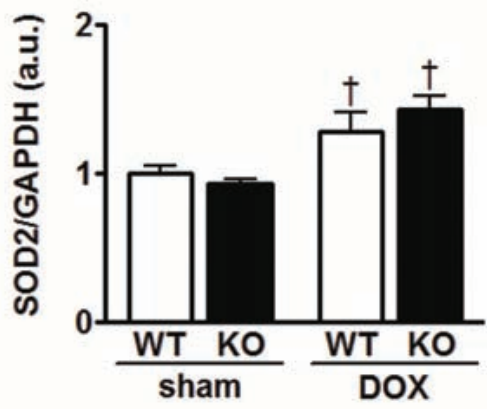

Figure 3. Oxidative stress and anti-oxidant mechanisms are equally induced in DOX-treated TSP-2-KO and WT mice. (A-D) Immunohistochemical staining for 3-nitrotyrosine demonstrated the presence of peroxynitrite in WT and TSP-2-KO hearts. (C, D) Oxidative stress was induced after DOX, but to a similar extent in both WT and TSP-2-KO hearts. (E, F) Transcript levels of the antioxidants catalase-1 (CAT) and superoxide dismutase-2 (SOD2) were significantly increased after DOX in WT $(n=15)$ and TSP-2-KO $(n=8)$ mice, but to an equal extent. Data are expressed in arbitrary units (a.u.) and presented as mean $\pm \mathrm{SEM},+P<0.05, \mathrm{DOX}-$ treated versus sham-treated mice with the same genotype. Scale bars represent $100 \mu \mathrm{m}$.

\section{Absence of TSP-2 and DOX treatment impaired Akt signaling in vivo and in vitro}

TSP-2 expression is significantly induced in WT mice upon chronic DOX exposure (Figure $4 \mathrm{~A}$ ). Akt plays a crucial role in protection against myocyte injury ${ }^{19}$ and acts downstream of the TSP receptor CD47/integrin associated protein (CD47/IAP) and beta1 integrin $^{20}$. Interestingly, Akt activity is significantly decreased in sham-treated TSP-2-KO compared to WT hearts of both male (Akt, arbitrary units; WT: $1.15 \pm 0.06$ versus TSP$2-K O, 0.56 \pm 0.03$ ) and female (Akt, arbitrary units; WT: $0.89 \pm 0.10$ versus TSP-2-KO, $0.43 \pm 0.05)$ mice. In addition, DOX alone blunted the phosphorylation of Akt in WT hearts, and impaired Akt signaling even more when TSP-2 was absent (Figure 4B), both independently from gender (Akt, arbitrary units; WT: female, $0.73 \pm 0.13$ versus male, $0.86 \pm 0.12, P=\mathrm{NS}$; and KO: female $0.10 \pm 0.03$ versus male, $0.38 \pm 0.19, P=\mathrm{NS})$. These results indicate that TSP-2 protects against DOX-cardiotoxicity by facilitating Akt activa- 
tion in cardiomyocytes. In line with these results, a $\sim 40$ percent reduction of TSP-2 expression (siTSP-2) in cardiomyocytes in vitro significantly decreased the activation of Akt (Figure 4C and D). In contrast to chronic DOX treatment in vivo, acute exposure to DOX in vitro caused a significant $\sim 70$ percent reduction of TSP-2 expression in control cardiomyocytes, with a concomitant decrease in Akt activation (Figure 4C and D). Combination of both DOX and SiTSP-2 did not further reduce TSP-2 protein levels compared to DOX alone, and resulted in a similar reduction of Akt activation (Figure $4 \mathrm{C}$ and D). Thus, increased DOX cardiotoxicity in cardiomyocytes may result from reduced TSP2 levels and thereby prevent proper Akt activation in these cells.

Akt-mediated TSP-2 expression has been reported in Akt-KO mice. ${ }^{21}$ To study whether impaired Akt activity in our DOX-model also reduced TSP-2 expression in cardiomyocytes, TSP-2 protein levels were determined in vitro after inhibition of Akt phosphorylation. A $52 \pm 4$ percent reduction in Akt phosphorylation caused a $85 \pm 1$ percent decrease in TSP-2 protein expression after 24 hours (Figure 4E and F), linking impaired activation of Akt to decreased protein expression levels of TSP-2. These findings point towards an inhibitory feedback mechanism between suppressed activation of Akt and reduced TSP-2 expression in cardiomyocytes after DOX. 
Heart, in vivo

A

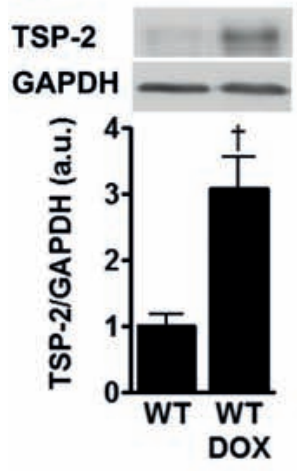

B

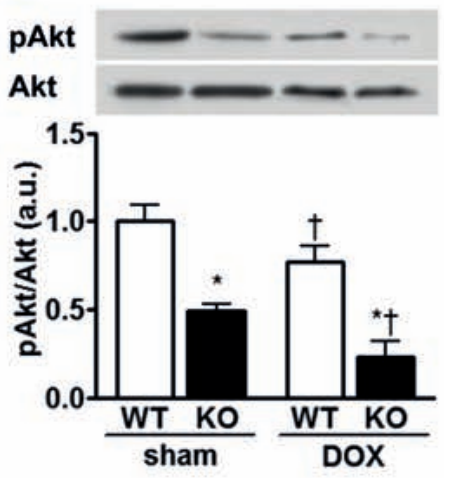

C D
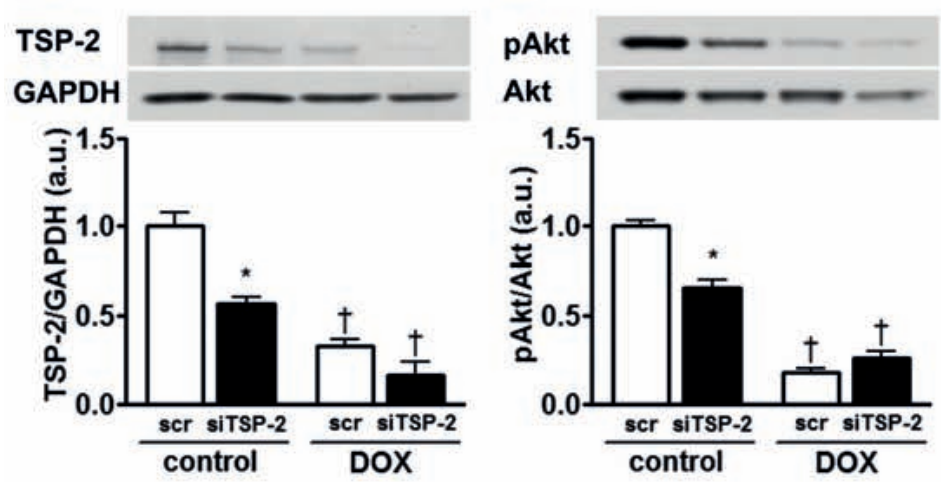

Cardiomyocyte, in vitro

E
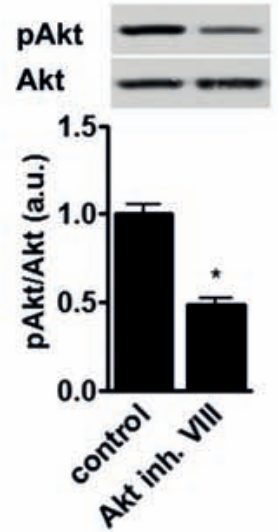

$F$

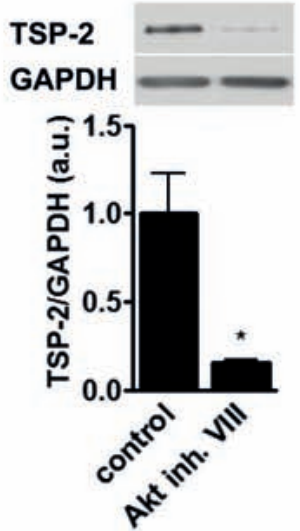


Figure 4. Impaired Akt activation and reduced TSP-2 expression in cardiomyocytes after DOX are enhanced by the regulatory feedback mechanism between Akt and TSP-2. (A) Immunoblotting showed enhanced TSP-2 expression in WT mice after DOX. (B) DOX represses Akt activation in both WT and TSP-2KO mice. Moreover, Akt activation was significantly impaired in TSP-2-KO mice compared to WT in both control and DOX-treated hearts ( $n=5$ mice per group). ${ }^{*} P<0.05$, TSP-2-KO versus WT mice within the same experimental group. $+P<0.05$, DOX- versus sham-treated mice with the same genotype. (C and D) SiTSP-2 significantly decreased activation of Akt in cardiomyocytes in vitro. DOX treatment alone also significantly decreased TSP-2 expression and Akt phosphorylation, but did not further reduce Akt activation when it was combined with siTSP-2 ( $n=3$ per condition). ${ }^{*} P<0.05$, siTSP-2 versus scrambled siRNA within the same experimental group. $+P<0.05$, DOX- versus saline-treated cardiomyocytes. (E and F) Pharmacological inhibition of Akt phosphorylation in cardiomyocytes in vitro reduced the expression of TSP-2 ( $\mathrm{n}=4$ per group), indicating that the extent of Akt activation regulates the expression of TSP-2 in cardiomyocytes. ${ }^{*} P<0.05$, Akt VIII inhibitor versus saline-treated NRCMs. Scr indicates scrambled siRNA, and siTSP-2 indicates siRNA against TSP-2 of rat origin. Data are expressed in arbitrary units (a.u.) and presented as mean \pm SEM.

Increased MMP-2 levels in TSP-2-KO mice contribute to enhanced matrix disruption after DOX

Ultrastructural examination after DOX administration revealed enhanced matrix disruption, characterized by disorganization of interstitial collagen fibers, in the hearts of TSP-2-KO mice compared to WT hearts, whereas no differences were observed in sham-treated animals (Figure 5A-D). Given the fact that DOX-cardiotoxicity not solely involves loss of cardiomyocytes, but also includes extensive cardiac remodeling, we investigated possible changes in the balance between MMPs and their inhibitors (TIMPs) in DOX-treated TSP-2-KO mice. RT-PCR analysis revealed significant higher MMP-2 mRNA levels in DOX-treated TSP-2-KO mice compared to WT mice (Table 3). MMP-9 transcription on the other hand was equally induced in WT and TSP-2-KO mice after DOX, but this was not significantly different between both genotypes. Importantly, expression of the natural inhibitor of MMP-2, TIMP2, was significantly reduced after DOX in WT and TSP-2-KO hearts, whereas transcript levels of TIMP-1, TIMP-3, and TIMP-4 were unaltered by DOX-administration (Table 3). Analysis of MMP-2 and MMP9 protein levels in these hearts by zymography further support these observations by showing that DOX significantly increased MMP-2 and MMP-9 levels in TSP-2-KO hearts compared to sham-treated TSP-2-deficient hearts (Figure 5E-G). MMP-2 levels in DOXtreated WT mice were significantly lower compared to non-treated WT mice, while increased MMP-2 levels were measured in the absence of TSP-2 after DOX (Figure 5E and F). Similar to MMP-9 mRNA expression, MMP-9 protein levels were equally induced in DOX-treated TSP-2-KO compared to WT hearts (Figure 5E and G), indicating that TSP-2 controls DOX-induced MMP-2 but not MMP-9 levels. 
Table 3. Relative transcript levels of MMPs and TIMPs

\begin{tabular}{lcccc}
\hline & \multicolumn{2}{c}{ Sham, 24 weeks of age } & \multicolumn{2}{c}{ 12 weeks DOX, 24 weeks of age } \\
\cline { 2 - 5 } & $\begin{array}{c}\text { WT } \\
(\mathrm{n}=15)\end{array}$ & $\begin{array}{c}\text { KO } \\
(\mathrm{n}=10)\end{array}$ & $\begin{array}{c}\text { WT } \\
(\mathrm{n}=14)\end{array}$ & $\begin{array}{c}\text { KO } \\
(\mathrm{n}=11)\end{array}$ \\
\hline MMP-2 & $0.57 \pm 0.10$ & $0.55 \pm 0.04$ & $0.49 \pm 0.05$ & $0.81 \pm 0.17^{*}$ \\
MMP-9 & $0.34 \pm 0.06$ & $0.38 \pm 0.04$ & $0.51 \pm 0.08$ & $0.65 \pm 0.18$ \\
TIMP-1 & $0.28 \pm 0.05$ & $0.29 \pm 0.05$ & $0.22 \pm 0.03$ & $0.18 \pm 0.08$ \\
TIMP-2 & $0.74 \pm 0.06$ & $0.88 \pm 0.10$ & $0.53 \pm 0.04^{\dagger}$ & $0.55 \pm 0.05^{\dagger}$ \\
TIMP-3 & $0.86 \pm 0.12$ & $0.77 \pm 0.17$ & $0.86 \pm 0.09$ & $0.88 \pm 0.17$ \\
TIMP-4 & $0.44 \pm 0.05$ & $0.36 \pm 0.05$ & $0.35 \pm 0.05$ & $0.32 \pm 0.04$ \\
\hline
\end{tabular}

Data are expressed as mean \pm SEM. ${ }^{*} P<0.05$, KO versus WT mice within the same experimental group. ${ }^{\dagger} P<0.05,24$ weeks old DOX-treated mice versus 24 weeks old sham mice, with the same genotype.

Heart, in vivo

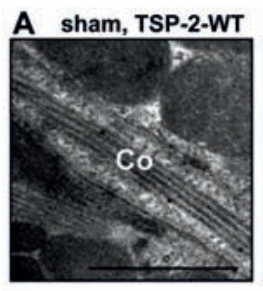

E

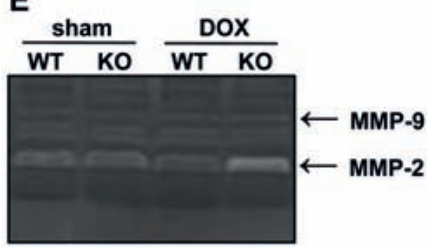

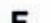

F

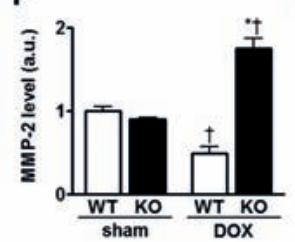

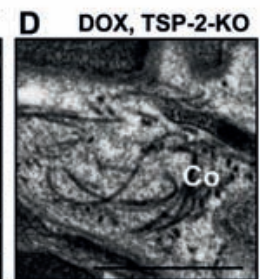

G

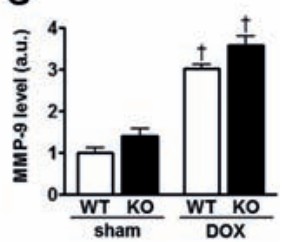

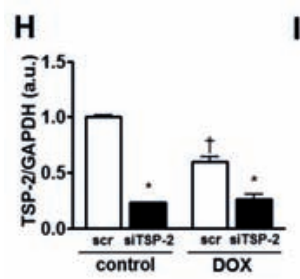

\section{Cardiomyocyte, in vitro}

I
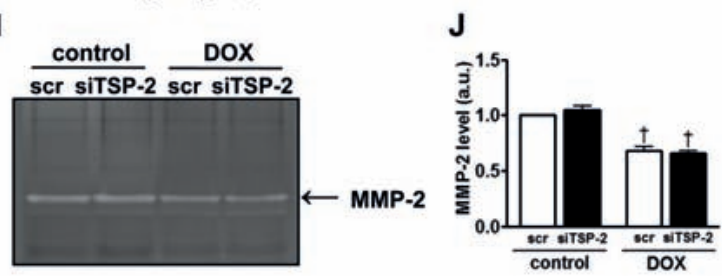

\section{Cardiac fibroblast, in vitro}

K

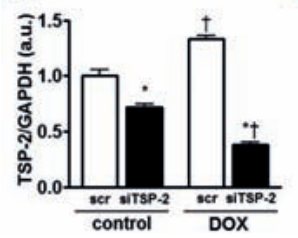

L

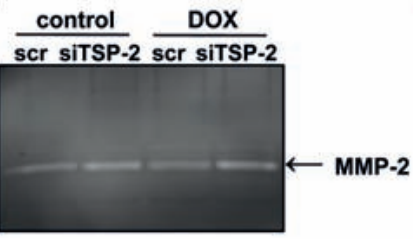

M

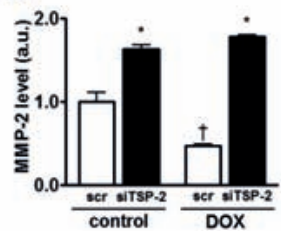


Figure 5. Increased fibroblast-driven MMP-2 levels are independent from Akt signaling and contributed to the decreased matrix integrity in TSP-2-KO mice after DOX. (A-D) Ultrastructural analysis unveiled decreased ECM integrity in TSP-2-KO mice after DOX, characterized by increased matrix disruption and abnormal organization of collagens. Co, indicates collagen. Scale bars represent $1 \mu \mathrm{m}$. (E-G) Representative zymogram (E) shows that DOX significantly increased MMP-2 levels in TSP-2-KO mice, but not in WT animals (F), whereas MMP-9 activity increased in both WT and TSP-2-KO mice (G). Data are expressed in arbitrary units (a.u.) and presented as mean $\pm \mathrm{SEM}, \mathrm{n}=6$ per group. ${ }^{*} P<0.05$, TSP-2-KO versus WT mice within the same experimental group. $+P<0.05$, DOX-versus sham-treated mice with the same genotype. $(H-$ M) SiTSP-2 repressed TSP-2 transcript levels in cardiomyocytes and cardiac fibroblasts. DOX alone increased TSP-2 expression in cardiac fibroblasts, but not in cardiomyocytes in vitro. Representative zymograms demonstrate that treatment with siTSP-2 or DOX was not sufficient to affect MMP-2 levels in cardiomyocytes, but in scr-treated cardiac fibroblasts increased TSP-2 expression after DOX was accompanied by decreased MMP-2 activity. scr indicates scrambled siRNA, and siTSP-2 indicates siRNA against TSP-2 of rat origin. Data are expressed in arbitrary units (a.u.) and presented as mean $\pm \mathrm{SEM}, \mathrm{n}=3$ per group. ${ }^{*} P<0.05$, siTSP-2 versus scrambled siRNA within the same experimental group. $+P<0.05$, DOX versus saline-treated cardiomyocytes or fibroblasts.

To investigate the distinct responses in the induction of MMP-2 in cardiomyocytes and cardiac fibroblasts, relative MMP-2 levels were quantified in the lysates of siTSP-2 transfected cardiomyocytes and fibroblasts after in vitro DOX treatment (Figure $5 \mathrm{H}-\mathrm{M}$ ). Decreased TSP-2 expression in non-treated cardiomyocytes was not sufficient to cause a difference in MMP-2 levels between control and siTSP-2 transfected cardiomyocytes, nor did DOX-induced repression of TSP-2 expression result in a difference in MMP-2 induction (Figure $5 \mathrm{H}-\mathrm{J}$ ). In contrast to cardiomyocytes, transcriptional repression of TSP-2 in cardiac fibroblast increased MMP-2 levels. More importantly, DOX-exposure of scrambled RNA transfected fibroblasts increased TSP-2 expression with a concomitant decrease in MMP-2 levels, while additional repression of TSP-2 by DOX did not further increased MMP-2 levels compared to non-treated siTSP-2 fibroblasts (Figure $5 \mathrm{~K}-\mathrm{M})$. Thus, the response to DOX in terms of the induction of MMP-2 in vivo resemble the observed response in fibroblasts, but not in cardiomyocytes, in vitro. Activation of MMPs has been linked to the induction of oxidative stress. ${ }^{8,10}$ We investigated whether reduced TSP-2 expression was associated with the amount oxidative stress in cardiac fibroblasts, which might explain the differences in MMP-2 induction in these cells after DOX. RT-PCR analysis showed that transcript levels of the antioxidant GPX1, and Rac1, all associated with oxidative stress, were not differently induced in siTSP-2 treated fibroblasts after DOX (Supplemental figure 3A and B).

Finally, inhibition of Akt signaling did not alter MMP-2 levels in cardiomyocytes and cardiac fibroblasts in vitro, indicating that increased induction of MMP-2 after DOX was independent of Akt signaling (Supplemental figure 4A and B). Together these data indicate that DOX-cardiotoxicity also affects cardiac fibroblasts via the induction of MMPs, however this seems unrelated to the amount of oxidative stress. 


\section{Discussion}

DOX is extremely effective in the treatment of many cancers, however its cardiotoxicity severely limits its clinical utilization as a antineoplastic agent. Although it is well established that DOX cardiotoxicity involves cardiac injury and remodeling, most studies solely addressed the cardiomyocyte effects, leaving the role of the ECM relatively unexplored. The present study is the first to provide evidence for the implication of a matrix element in cardiac injury and remodeling following DOX-cardiotoxicity. The absence of TSP-2 renders the heart more susceptible to apoptosis of cardiomyocytes and disruption of the ECM after chronic DOX treatment. Impaired Akt signaling and enhanced MMP-2 activity contributed to cardiac injury and dysfunction in TSP-2-KO mice after DOX.

First, we addressed whether differences in the induction of oxidative stress in the absence of TSP-2 could have been the cause of increased cardiac damage. Yet the degree of oxidative stress and antioxidant induction did not significantly differ in TSP2-KO compared to WT DOX-hearts. Despite similar oxidative stress, cardiomyocyte damage and dropout and resulting reparative fibrosis was more pronounced in the absence of TSP-2. Thus TSP-2 affects the damage response to oxidative stress, and not the production of reactive oxygen species itself.

A protective role for TSP-2 against DOX-cardiotoxicity may be mediated by regulating outward-inward cell signaling and function. Impaired Akt survival signaling has previously been implicated as a key feature of DOX-induced cell damage. ${ }^{22,23}$ Here, both DOX treatment or TSP-2 knockdown in vitro or its absence in vivo significantly reduced the activation of Akt in cardiomyocytes. Reduced Akt activation as a cause of cardiomyocyte damage in response to DOX is in line with previous reports, showing that restoration of Akt signaling by increasing the cardioprotective heat shock protein $20^{3}$, erythropoietin ${ }^{4}$ or glycoprotein- $130^{24}$ also hinders DOX-induced myocyte damage. However, the extrapolation of the protective role of Akt in cardiomyocyte survival in mice to other species is unsure, as it was shown that Akt does not have a central role in cardiac protection against ischemic injury in pigs. ${ }^{25}$

Beside a reduction of TSP-2 expression and impaired Akt activation in DOX-treated cardiomyocytes, TSP-2 expression per se decreases when Akt phosphorylation is inhibited. This suggests a regulatory mechanism between the extent of Akt activation and TSP-2 expression in cardiomyocytes. These findings are in parallel with reduced TSP-2 levels in endothelial cells of Akt-KO mice, where TSP-2 expression could be restored by re-expression of Akt. ${ }^{21}$ Thus, the cytotoxicity of DOX in cardiomyocytes involves the reduction of Akt activity and subsequent down-regulation of TSP-2, whereas the latter functions as a negative feedback mechanism that further impairs Akt survival signaling. This negative feedback-loop contributes to the increased susceptibility to DOXcardiotoxicity of cardiomyocytes in the absence of TSP- 2 .

In addition to the adverse cardiomyocyte effects in the absence of TSP-2, DOX also induces a strong collagen-related phenotype, indicated by the intramyocardial bleed- 
ing and increased fibrosis in DOX-treated TSP-2-KO mice. Cardiac rupture is associated with an imbalance between MMP and TIMP activities in the heart. Moreover, TIMP-3KO mice show decreased collagen synthesis and enhanced MMP activities following myocardial infarction, resulting in increased cardiac rupture. ${ }^{26}$ TIMP-1, $-3,-4$ and MMP9 transcript levels were not significantly different in WT and TSP-2-KO hearts after DOX. Here we show that TSP-2 also protects against matrix degradation during DOXinduced cardiotoxicity by decreasing MMP-2 activity. ${ }^{27}$ Enhanced MMP-2 activity may cause myocardial damage by increasing matrix disruption and cardiac dysfunction ${ }^{28}$, or by the degradation of thin and thick myofilaments of the cardiomyocyte sarcomeres. ${ }^{29-}$ ${ }^{31}$ Importantly, TSP-2 knockdown only increases MMP-2 levels in cardiac fibroblasts but not in cardiomyocytes in vitro. This effect reflects the cardiac fibroblast restricted induction of TSP-2 upon DOX-exposure in vitro. Increased TSP-2 levels in fibroblasts counteract the function of MMP-2, thereby blunting its matrix degrading activity. The fact that increased TSP-2 expression together with reduced MMP-2 levels after DOX in cardiac fibroblasts in vitro resembles the effects in WT mice after DOX, implies that the cardiac fibroblasts are responsible for the dynamic upregulation of MMP-2 activity in DOX-treated TSP-2-KO hearts. The fact that this matrix preserving effect predominantly relies on a cardiac fibroblast-driven mechanism is supported by the notion that another matricellular protein, biglycan, which interacts with collagen fibrils and protects against ECM degradation by MMPs, is regulated in fibroblasts during cardiac remodelling. ${ }^{32}$ In addition, mice deficient for biglycan show intramyocardial bleeding and rupture in response to myocardial infarction similar to DOX treatment in TSP-2-KO mice. $^{33}$ Thus, beside decrease Akt activation in cardiomyocytes, increased MMP-2 activity by fibroblasts represents a second mechanism of increased DOX-CMP in the absence of TSP-2.

Both decreased Akt-survival signaling and enhanced myocyte dropout, as well as increased matrix remodeling was most pronounced in male TSP-2-KO mice. These results are somewhat surprising, as female gender is considered a risk factor for DOX cardiotoxicity in humans. $^{34,35}$ Gender differences observed may result of the generally increased predisposition of males compared to females in mice to develop heart failure in response to ischemia ${ }^{36,37}$, hypertension or myocarditis ${ }^{38-40}$, in addition to the early onset of the aging phenotype in male TSP-2-KO mice around the age of twentyfour weeks. ${ }^{16}$ So, accelerated aging and increased male-related susceptibility for heart failure in mice may exacerbate the DOX-induced dropout of cardiomyocytes and resulting fibrosis, as well as the induction of MMPs, explaining the cardiac dysfunction and enhanced mortality observed in male TSP-2-KO mice. For this reason it is not surprising that the effects of DOX seemed to be much more pronounced in male TSP-2-KO mice.

In conclusion, the absence of TSP-2 results in progressive development of DOX$\mathrm{CMP}$ by enhancing cardiomyocyte damage and matrix disruption. The novel finding that one simple matrix element may mediate DOX-CMP points towards new avenues 
for exploring better interventions for the prevention of cardiotoxicity of DOX chemotherapy.

\section{Acknowledgments}

The authors thank Hans Duimel (Maastricht University Medical Center, Department of Pathology, Cardiovascular Research Institute Maastricht, Maastricht, the Netherlands) for excellent technical support and Paul Veulemans (Department of Cardiovascular diseases, K.U. Leuven, Leuven, Belgium) for echocardiographic analysis. 


\section{References}

1 Chen X, Chen Y, Bi Y, Fu N, Shan C, Wang S, Aslam S, Wang PW, Xu J. Preventive cardioprotection of erythropoietin against doxorubicin-induced cardiomyopathy. Cardiovasc Drugs Ther. 2007;21(5):367374.

2 Doroshow JH, Davies KJ. Redox cycling of anthracyclines by cardiac mitochondria. II. Formation of superoxide anion, hydrogen peroxide, and hydroxyl radical. J Biol Chem. 1986;261(7):3068-3074.

3 Fan GC, Zhou X, Wang X, Song G, Qian J, Nicolaou P, Chen G, Ren X, Kranias EG. Heat shock protein 20 interacting with phosphorylated Akt reduces doxorubicin-triggered oxidative stress and cardiotoxicity. Circ Res. 2008;103(11):1270-1279.

4 Kim KH, Oudit GY, Backx PH. Erythropoietin protects against doxorubicin-induced cardiomyopathy via a phosphatidylinositol 3-kinase-dependent pathway. J Pharmacol Exp Ther. 2008;324(1):160-169.

5 Mukhopadhyay P, Rajesh M, Batkai S, Kashiwaya Y, Hasko G, Liaudet L, Szabo C, Pacher P. Role of superoxide, nitric oxide, and peroxynitrite in doxorubicin-induced cell death in vivo and in vitro. Am J Physiol Heart Circ Physiol. 2009;296(5):H1466-1483.

6 Spallarossa P, Garibaldi S, Altieri P, Fabbi P, Manca V, Nasti S, Rossettin P, Ghigliotti G, Ballestrero A, Patrone F, Barsotti A, Brunelli C. Carvedilol prevents doxorubicin-induced free radical release and apoptosis in cardiomyocytes in vitro. J Mol Cell Cardiol. 2004;37(4):837-846.

7 Caulfield JB, Bittner V. Cardiac matrix alterations induced by adriamycin. Am J Pathol. 1988;133(2):298305.

8 Bai P, Mabley JG, Liaudet L, Virag L, Szabo C, Pacher P. Matrix metalloproteinase activation is an early event in doxorubicin-induced cardiotoxicity. Oncol Rep. 2004;11(2):505-508.

9 Goetzenich A, Hatam N, Zernecke A, Weber C, Czarnotta T, Autschbach R, Christiansen S. Alteration of matrix metalloproteinases in selective left ventricular adriamycin-induced cardiomyopathy in the pig. J Heart Lung Transplant. 2009;28(10):1087-1093.

10 Kizaki K, Ito R, Okada M, Yoshioka K, Uchide T, Temma K, Mutoh K, Uechi M, Hara Y. Enhanced gene expression of myocardial matrix metalloproteinases 2 and 9 after acute treatment with doxorubicin in mice. Pharmacol Res. 2006;53(4):341-346.

11 Dudnakova TV, Lakomkin VL, Tsyplenkova VG, Shekhonin BV, Shirinsky VP, Kapelko VI. Alterations in myocardial cytoskeletal and regulatory protein expression following a single Doxorubicin injection. J Cardiovasc Pharmacol. 2003;41(5):788-794.

12 Bornstein P, Sage EH. Matricellular proteins: extracellular modulators of cell function. Curr Opin Cell Biol. 2002;14(5):608-616.

13 Lange-Asschenfeldt B, Weninger W, Velasco P, Kyriakides TR, von Andrian UH, Bornstein P, Detmar M. Increased and prolonged inflammation and angiogenesis in delayed-type hypersensitivity reactions elicited in the skin of thrombospondin-2--deficient mice. Blood. 2002;99(2):538-545.

14 Streit M, Riccardi L, Velasco P, Brown LF, Hawighorst T, Bornstein P, Detmar M. Thrombospondin-2: a potent endogenous inhibitor of tumor growth and angiogenesis. Proc Natl Acad Sci U $S$ A. 1999;96(26):14888-14893.

15 Schroen B, Heymans S, Sharma U, Blankesteijn WM, Pokharel S, Cleutjens JP, Porter JG, Evelo CT, Duisters R, van Leeuwen RE, Janssen BJ, Debets JJ, Smits JF, Daemen MJ, Crijns HJ, Bornstein P, Pinto YM. Thrombospondin-2 is essential for myocardial matrix integrity: increased expression identifies failureprone cardiac hypertrophy. Circ Res. 2004;95(5):515-522.

16 Swinnen M, Vanhoutte D, Van Almen GC, Hamdani N, Schellings MW, D’Hooge J, Van der Velden J, Weaver MS, Sage EH, Bornstein P, Verheyen FK, VandenDriessche T, Chuah MK, Westermann D, Paulus WJ, Van de Werf F, Schroen B, Carmeliet P, Pinto YM, Heymans S. Absence of thrombospondin-2 causes age-related dilated cardiomyopathy. Circulation. 2009;120(16):1585-1597.

17 Kyriakides TR, Leach KJ, Hoffman AS, Ratner BD, Bornstein P. Mice that lack the angiogenesis inhibitor, thrombospondin 2, mount an altered foreign body reaction characterized by increased vascularity. Proc Natl Acad Sci U S A. 1999;96(8):4449-4454. 
18 Khan M, Varadharaj S, Shobha JC, Naidu MU, Parinandi NL, Kutala VK, Kuppusamy P. C-phycocyanin ameliorates doxorubicin-induced oxidative stress and apoptosis in adult rat cardiomyocytes. J Cardiovasc Pharmacol. 2006;47(1):9-20.

19 Fujio Y, Nguyen T, Wencker D, Kitsis RN, Walsh K. Akt promotes survival of cardiomyocytes in vitro and protects against ischemia-reperfusion injury in mouse heart. Circulation. 2000;101(6):660-667.

20 Bouchard V, Harnois C, Demers MJ, Thibodeau S, Laquerre V, Gauthier R, Vezina A, Noel D, Fujita N, Tsuruo T, Arguin M, Vachon PH. B1 integrin/Fak/Src signaling in intestinal epithelial crypt cell survival: integration of complex regulatory mechanisms. Apoptosis. 2008;13(4):531-542.

21 Chen J, Somanath PR, Razorenova O, Chen WS, Hay N, Bornstein P, Byzova TV. Akt1 regulates pathological angiogenesis, vascular maturation and permeability in vivo. Nat Med. 2005;11(11):1188-1196.

22 Lee ER, Kim JY, Kang YJ, Ahn JY, Kim JH, Kim BW, Choi HY, Jeong MY, Cho SG. Interplay between PI3K/Akt and MAPK signaling pathways in DNA-damaging drug-induced apoptosis. Biochim Biophys Acta. 2006;1763(9):958-968.

23 Zhao Y, You H, Yang Y, Wei L, Zhang X, Yao L, Fan D, Yu Q. Distinctive regulation and function of PI 3K/Akt and MAPKs in doxorubicin-induced apoptosis of human lung adenocarcinoma cells. J Cell Biochem. 2004;91(3):621-632.

24 Negoro S, Oh H, Tone E, Kunisada K, Fujio Y, Walsh K, Kishimoto T, Yamauchi-Takihara K. Glycoprotein 130 regulates cardiac myocyte survival in doxorubicin-induced apoptosis through phosphatidylinositol 3kinase/Akt phosphorylation and Bcl-xL/caspase-3 interaction. Circulation. 2001;103(4):555-561.

25 Skyschally A, van Caster P, Boengler K, Gres P, Musiolik J, Schilawa D, Schulz R, Heusch G. Ischemic postconditioning in pigs: no causal role for RISK activation. Circ Res. 2009;104(1):15-18.

26 Hammoud L, Lu X, Lei M, Feng Q. Deficiency in TIMP-3 increases cardiac rupture and mortality postmyocardial infarction via EGFR signaling: beneficial effects of cetuximab. Basic Res Cardiol. 2011;106(3):459-471.

27 Yang Z, Strickland DK, Bornstein P. Extracellular matrix metalloproteinase 2 levels are regulated by the low density lipoprotein-related scavenger receptor and thrombospondin 2. J Biol Chem. 2001;276(11):8403-8408.

28 Cleutjens J, Huynen F, Smits J, Bornstein P, Daemen M. Thrombospondin-2 deficiency in mice results in cardiac rupture early after myocardial infarction. Circ Res. 1999;100 (Suppl.):156.

29 Sawicki G, Leon H, Sawicka J, Sariahmetoglu M, Schulze CJ, Scott PG, Szczesna-Cordary D, Schulz R. Degradation of myosin light chain in isolated rat hearts subjected to ischemia-reperfusion injury: a new intracellular target for matrix metalloproteinase-2. Circulation. 2005;112(4):544-552.

30 Wang W, Schulze CJ, Suarez-Pinzon WL, Dyck JR, Sawicki G, Schulz R. Intracellular action of matrix metalloproteinase-2 accounts for acute myocardial ischemia and reperfusion injury. Circulation. 2002;106(12):1543-1549.

31 Bergman MR, Teerlink JR, Mahimkar R, Li L, Zhu BQ, Nguyen A, Dahi S, Karliner JS, Lovett DH. Cardiac matrix metalloproteinase-2 expression independently induces marked ventricular remodeling and systolic dysfunction. Am J Physiol Heart Circ Physiol. 2007;292(4):H1847-1860.

32 Tiede K, Melchior-Becker A, Fischer JW. Transcriptional and posttranscriptional regulators of biglycan in cardiac fibroblasts. Basic Res Cardiol. 2010;105(1):99-108.

33 Westermann D, Mersmann J, Melchior A, Freudenberger T, Petrik C, Schaefer L, Lullmann-Rauch R, Lettau O, Jacoby C, Schrader J, Brand-Herrmann SM, Young MF, Schultheiss HP, Levkau B, Baba HA, Unger T, Zacharowski K, Tschope C, Fischer JW. Biglycan is required for adaptive remodeling after myocardial infarction. Circulation. 2008;117(10):1269-1276.

34 Lipshultz SE, Lipsitz SR, Mone SM, Goorin AM, Sallan SE, Sanders SP, Orav EJ, Gelber RD, Colan SD. Female sex and drug dose as risk factors for late cardiotoxic effects of doxorubicin therapy for childhood cancer. N Engl J Med. 1995;332(26):1738-1743.

35 Silber JH, Jakacki RI, Larsen RL, Goldwein JW, Barber G. Increased risk of cardiac dysfunction after anthracyclines in girls. Med Pediatr Oncol. 1993;21(7):477-479.

36 Chen Q, Williams R, Healy CL, Wright CD, Wu SC, O'Connell TD. An association between gene expression and better survival in female mice following myocardial infarction. J Mol Cell Cardiol. 2010;49(5):801811. 
37 Qiu H, Depre C, Vatner SF, Vatner DE. Sex differences in myocardial infarction and rupture. J Mol Cell Cardiol. 2007;43(5):532-534.

38 Buys ES, Sips P, Vermeersch P, Raher MJ, Rogge E, Ichinose F, Dewerchin M, Bloch KD, Janssens S, Brouckaert P. Gender-specific hypertension and responsiveness to nitric oxide in sGCalpha1 knockout mice. Cardiovasc Res. 2008;79(1):179-186.

39 Kawanishi H, Hasegawa Y, Nakano D, Ohkita M, Takaoka M, Ohno Y, Matsumura Y. Involvement of the endothelin ET(B) receptor in gender differences in deoxycorticosterone acetate-salt-induced hypertension. Clin Exp Pharmacol Physiol. 2007;34(4):280-285.

40 Li K, Xu W, Guo Q, Jiang Z, Wang P, Yue Y, Xiong S. Differential macrophage polarization in male and female BALB/c mice infected with coxsackievirus B3 defines susceptibility to viral myocarditis. Circ Res. 2009;105(4):353-364. 


\section{Chapter 4}

\section{Supplemental material}

\section{Expanded methods}

Transgenic mice and model of doxorubicin-induced cardiomyopathy

The present study conforms with the Guide for the care and use of laboratory animals published by the US National Institutes of Health (NIH Publication NO. 85-23, revised 1996) and was approved by the Institutional Animal Research Committee according to the rules formulated in the Dutch and Belgian law on care and use of experimental animals.

DOX-CMP was induced in 12 weeks old male and female TSP-2 knockout (KO) mice (male, $n=24$; female, $n=26$ ) and their wild-type (WT) littermates (male, $n=17$; female, $\mathrm{n}=15$ ) on a C57BL6/129SvJ/EMS+Ter genetic background. ${ }^{1}$ Sham TSP-2-KO (male, $n=11$; female, $n=9$ ) and WT (male, $n=14$; female, $n=10$ ) mice were treated with saline. A clinically relevant dose of $24 \mathrm{mg} / \mathrm{kg}$ DOX (Pfizer, \#32001013) was administered by a weekly intra-peritoneal injection of $2 \mathrm{mg} / \mathrm{kg}$ for 12 weeks. Cardiac function at baseline and the presence of DOX-CMP was assessed by echocardiographic examination after 12 weeks of DOX treatment. Subsequently, hearts were taken out and prepared for molecular, histological and ultrastructural evaluation. ${ }^{2,3}$

\section{Echocardiography}

Echocardiographic examination was performed at baseline and after exposure to a cumulative dose of $24 \mathrm{mg} / \mathrm{kg}$ DOX or saline, using a 13-MHz transducer (i13L, GE VingMed ultrasound; Horten, Norway) on a VingMed Vivid 7 scanner (GE VingMed ultrasound, Horten, Norway) in anesthetized ( $2 \%$ isoflurane) mice while kept at $37^{\circ} \mathrm{C}$. Echocardiographic images were taken in B-mode to analyse fractional shortening (FS), left ventricular diameters at end-diastole (LVEDd), left ventricular posterior wall at end-diastole (LVPWd), and intraventricular septal thickness at end-diastole (IVSd). ${ }^{3}$ The heart rate (HR) was calculated from M-mode echocardiographic images.

\section{Immunohistochemical and electron microscopic analysis}

For immunohistochemical examination, hearts were cut transversely, fixed in $1 \%$ buffered paraformaldehyde and embedded in paraffin. To study ventricular morphology, hearts were histochemically stained with hematoxylin and eosin. Collagen content was morphometrically determined as percentage sirius red staining area per total cardiac 
tissue area. Immunostaining was performed on $4 \mu \mathrm{m}$ thick paraffin sections using a primary antibody against 3-nitrotyrosine (Upstate, Lake Placid, NY, USA, \#06-284), desmin (Abcam), CD45 (BD Bioscience pharmingen), active cleaved caspase-3 (Cell Signaling Technology), and TUNEL (Chemicon). Active cleaved caspase-3 and TUNEL staining were presented as the percentage positive staining cardiomyocyte nuclei per total cardiomyocyte nuclei. The cardiomyocyte density was determined from 10 representative areas across the LV wall and cardiomyocyte area was measured as the mean of $>50$ cross-sectioned cardiomyocytes. In order to obtain a parameter for cardiomyocyte content of the left ventricle, cardiomyocyte density was multiplied by cardiomyocyte area. All morphometrical analyses were performed using a Leica DMRXE microscope (Leica Microsystems Cambridge Ltd) and Leica Qwin image processing and analysis software (Leica Microsystems Cambridge Ltd).

For electron microscopic examination, hearts were fixed in $2.5 \%$ glutaraldehyde in $0.1 \mathrm{M}$ phosphate buffer at $\mathrm{pH} 7.4$, and post-fixed in $1 \%$ osmiumtetroxide in $0.067 \mathrm{M}$ cacodylate buffer. After fixation, the samples were routinely dehydrated in graded ethanol series (70\%, $90 \%$ and $100 \%$ ), followed by embedding in Epon. Electron microscopic images were made from $80 \mathrm{~nm}$ thick sections with a Philips CM100 (F.E.I., Eindhoven, The Netherlands) at an accelerating voltage of $80 \mathrm{KV}$.

\section{Zymographic matrix metalloproteinase levels in DOX-CMP}

To determine zymographic levels of MMP-2 and MMP-9 in DOX-CMP, heart tissue was homogenized (RyboLyser, Hybaid, UK) and total proteins were extracted in homogenization buffer containing $50 \mathrm{mM}$ Tris- $\mathrm{HCL} \mathrm{pH} 7.5,10 \mathrm{mM} \mathrm{CaCl}$, and $10 \mu \mathrm{M} \mathrm{ZnCl}_{2}$. For measurement of zymographic levels in vitro NRCMs and NRCFs were harvested in 150 $\mu l$ 2x zymography buffer (Bio-Rad Laboratories Inc.). Protein concentrations were determined using the bicinchoninic acid (BCA) assay (Pierce, IL).

Zymographic levels of MMP-2 and MMP-9 (gelatinase activity) was determined as previously described. ${ }^{2,4}$ Briefly, $25 \mu \mathrm{g}$ protein was diluted in sample buffer $(0.04 \mathrm{M}$ Tris-HCl pH6.8, 5\% glycerol, $16.6 \mu \mathrm{g} / \mathrm{mL}$ SDS, and $2 \mathrm{mg}$ bromophenol blue) and loaded on a $0.1 \%$ gelatin gel in $10 \%$ Tris-glycine (NX EC6175, Sanver Tech, Bouchout, Belgium). Gel-electrophoresis was performed for two hours at 126 Volt and 40mA per gel in a buffer containing $24 \mathrm{mM}$ Tris pH8.3, $192 \mathrm{mM}$ glycine and 0.1\% SDS. After 30 minutes incubation in renaturation buffer $(2.5 \% \mathrm{v} / \mathrm{v}$ Triton X-100) and overnight incubation in developing buffer $(40 \mathrm{mM}$ Tris- $\mathrm{HCl} \mathrm{pH} \mathrm{7.6,2.0} \mathrm{M} \mathrm{NaCl,} 6.67 \mathrm{mM} \mathrm{CaCl}, 0.01 \%$ v/v Triton $\mathrm{X}-100)$ gels were stained for 3 hours in staining buffer $(0.5 \% \mathrm{w} / \mathrm{v}$ Coomassie Blue R250 in $45 \% \mathrm{v} / \mathrm{v}$ ethanol (EtOH), $10 \% \mathrm{v} / \mathrm{v}$ acetic acid) and destained by repeated stirring in destaining buffer (45\% v/v EtOH and $10 \%$ acetic acid). Next, gels were transferred to a storage buffer ( $7 \% \mathrm{v} / \mathrm{v}$ acetic acid, $25 \% \mathrm{v} / \mathrm{v}$ methanol and $2 \% \mathrm{v} / \mathrm{v}$ glycerol) and dried between cellophane membranes (Bio-Rad Laboratories Inc.). Gelatinase activity became visible as clear white bands in the blue stained gel. MMP levels were quantified 
by measuring the intensity and area of the white bands using Quantimed 600 image analysis software (Leica instruments, Hamburg, Germany).

\section{RNA extraction and analysis of transcript levels}

Total RNA was isolated from the left ventricles of sham- and DOX-treated WT and TSP2-KO mice, and siTSP-2 transfected primary neonatal rat cardiomyocytes (NRCMs) and cardiac fibroblasts (NRCFs), using the RNeasy Mini Kit (Qiagen, Hilden, Germany) according to the protocol of the manufacturers. Five hundred nanograms of total RNA was reverse transcribed into cDNA using the qScript cDNA synthesis kit (Quanta Biosciences, Gaithersburg, MD).

Transcript levels of catalase-1 (CAT1), superoxide dismutase-2 (SOD2), MMP-2 and -9 , TIMP-1, $-2,-3$, and -4 , glutathione peroxidase 1 (GPX1), Rac1 and TSP-2 were determined using a real-time fluorescence detection method. Each assay consisted of two unlabeled PCR primers and a 5'-end FAM TM dye and 3'-end TAMRA labeled TaqMan ${ }^{\circledR}$ probe, designed with primer Express Software (PE Applied Biosystems) or Assayon-demand primer/probe mix from Applied Biosystems (Assay-on-demand number: below Supplemental table 1). Optimal PCR conditions were created with $12.5 \mu \mathrm{I} 2 \mathrm{X}$ PCR Master Mix for Taqman TM assays (PE Applied Biosystems) with a final concentration of $5 \mathrm{mM} \mathrm{MgCl}_{2}, 300 \mathrm{nM}$ of each primer, $200 \mathrm{nM}$ probe, and $2 \mathrm{ng}$ cDNA-template. All transcript levels were determined in duplo and normalized for mouse or rat glyceraldehyde-3-phosphate dehydrogenase (GAPDH) expression in the same sample. cDNA was amplified through PCR on an Applied Biosystems 7500 Fast Real-Time PCR system (PE Applied Biosystems) ${ }^{2}$. Consecutive stages were followed: 2 minutes at $50^{\circ} \mathrm{C}, 10$ minutes at $95^{\circ} \mathrm{C}$, continued by 45 cycles of 15 seconds at $95^{\circ} \mathrm{C}$ and 45 seconds at $60^{\circ} \mathrm{C}$. Primer sequences are listed in Supplement table 1.

\section{In vitro experimental approaches}

Primary neonatal rat cardiomyocytes (NRCMs) and cardiac fibroblasts (NRCFs) were isolated from the hearts of 1- to 3-day-old Lewis rats (Janvier, Le Genest Saint Isle, France). ${ }^{5}$ Briefly, cardiomyocytes were isolated by collagenase digestion from the ventricles. After 60 minutes of preplating, the non-attached NRCMs were separated from the NRCFs and plated in gelatinized six-well plates and cultured overnight in Dulbecco's modified Eagle's medium (DMEM) (Invitrogen) supplemented with $10 \%$ horse serum (PAA), 5\% heat-inactivated newborn calf serum (PAA), M-199 (Invitrogen), glucose (Merck), gentamycin (Invitrogen), and Penicillin Streptomycin (Invitrogen). NRCMs were cultured in the presence of cytosine $\beta$-D-arabinofuranoside (Sigma, \#C1768) to suppress proliferation of non-cardiomyocytes. NRCFs were cultured in DMEM (Invitrogen), supplemented with $10 \%$ fetal bovine serum (PAA) and gentamycin (Invitrogen). 
Supplemental table 1. Real-Time PCR primers

\begin{tabular}{|c|c|c|}
\hline Gene & Primer & Sequence $5^{\prime}-3^{\prime}$ \\
\hline \multirow[t]{3}{*}{ Mouse-CAT } & $\mathrm{F}$ & cctggcatcggagccca \\
\hline & $\mathrm{R}$ & gtgtccgggtaggccaaaaag \\
\hline & probe & ccctgacaaaatgcttcaggccg \\
\hline \multirow[t]{2}{*}{ Rat-GPX1 } & $\mathrm{F}$ & gcgcagatacagcaggcgct \\
\hline & $\mathrm{R}$ & aggggttgctaggctgcttgga \\
\hline \multirow[t]{2}{*}{ Rat-Rac1 } & $\mathrm{F}$ & caggggctagccatggcgaaa \\
\hline & $\mathrm{R}$ & agggggacagagaacggctcg \\
\hline \multirow[t]{2}{*}{ Mouse-MMP-2 } & $\mathrm{F}$ & ccgaggactatgaccgggata \\
\hline & $\mathrm{R}$ & caccttctgaatttccaccca \\
\hline \multirow[t]{2}{*}{ Mouse-MMP-9 } & $\mathrm{F}$ & cgagggcacgaggagctag \\
\hline & $\mathrm{R}$ & cgatcttcttcacggttgca \\
\hline \multirow[t]{2}{*}{ Mouse-TIMP-1 } & $\mathrm{F}$ & tcatggaaagcctctgtggat \\
\hline & $\mathrm{R}$ & cggcccgtgatgagaaact \\
\hline \multirow[t]{2}{*}{ Mouse-TIMP-2 } & $\mathrm{F}$ & tgccctgggacacgctta \\
\hline & $\mathrm{R}$ & actcacagcccatctggtacct \\
\hline \multirow[t]{2}{*}{ Mouse-TIMP-3 } & $\mathrm{F}$ & gccttctgcaactccgaca \\
\hline & $\mathrm{R}$ & ccctccttcaccagcttcttt \\
\hline \multirow[t]{2}{*}{ Mouse-TIMP-4 } & $\mathrm{F}$ & accaggcccagcactatgtc \\
\hline & $\mathrm{R}$ & gaggtgtaggtggcctgcgta \\
\hline \multirow[t]{2}{*}{ Rat-TSP-2 } & $\mathrm{F}$ & gaaatggtctacttctcagacctcaag \\
\hline & $\mathrm{R}$ & gcacactgctggagctgga \\
\hline \multirow[t]{3}{*}{ Mouse-GAPDH $^{\mathrm{a}}$} & $\mathrm{F}$ & acgtgccgcctggaga \\
\hline & $\mathrm{R}$ & ccctcagatgcctgcttca \\
\hline & probe & caccttcttgatgtcatcatacttggcagg \\
\hline \multirow[t]{2}{*}{ Rat-GAPDH $^{b}$} & $\mathrm{~F}$ & ggtggacctcatggcctaca \\
\hline & $\mathrm{R}$ & Ctctcttgctctcagtatccttgct \\
\hline
\end{tabular}

${ }^{\mathrm{a}}$ Housekeeping gene: GAPDH, glyceraldehyde-3-phosphate dehydrogenase used for normalization of genes from mouse origin after RT-PCR

${ }^{b}$ Housekeeping gene: GAPDH, glyceraldehyde-3-phosphate dehydrogenase used for normalization of genes from rat origin after RT-PCR

Mouse-SOD2 ID: Mm00449726_m1

For siRNA transfection $7.5 \times 10^{5}$ NRCMs, and $1.5 \times 10^{5}$ NRCFs per well were plated in six well plates. One day after isolation cells were placed in DMEM, containing low serum, for 24 hours before the medium was changed to serum-free DMEM without antibiotics for 4 hours. NRCMs and NRCFs were then transfected with $80 \mathrm{~nm}$ siTSP-2 (Dharmacon ON-TARGET plus smart-pool; \#L-081165-01) or scrambled RNA (Dharmacon ONTARGET plus control pool; \#D-001810-10-5), using lipofectamine 2000 (Invitrogen; \#11668). After 48 hours, NRCMs and NRCFs were exposed to $1 \mu \mathrm{M}$ DOX (Pfizer, \#32001013), or an equal volume of phosphate buffered saline for a subsequent 24 hours, when the cells were harvested for protein and RNA analysis.

To study the effect of Akt activation on the expression of TSP-2, NRCMs were cultured 24 hours under serum-free conditions in the presence of $10 \mu \mathrm{M}$ Akt-inhibitor VIII 
(Calbiochem, \#124017). After 24 hours of exposure, cells were harvested for protein expression analysis.

\section{Immunoblotting}

Left ventricular heart tissue was homogenized (RyboLyser, Hybaid, UK) and extracted in homogenization buffer containing $50 \mathrm{mM}$ Tris- $\mathrm{HCl} \mathrm{pH} \mathrm{7.5,} 10 \mathrm{mM} \mathrm{CaCl}_{2}$, and $10 \mu \mathrm{M}$ $\mathrm{ZnCl}_{2}$. Prior to SDS-PAGE the protein lysates were diluted $1: 1$ in $2 x$ sample buffer $(0.5$ $\mathrm{M}$ Tris- $\mathrm{HCl} \mathrm{pH} 6.8,20 \%$ glycerol, 4\% SDS, $1 \mathrm{mg}$ bromophenol blue, $\mathrm{H}_{2} \mathrm{O}$, and 2mercaptoethanol) and boiled for 5 minutes at $95^{\circ} \mathrm{C}$. NRCMs were harvested in a identical manner. The protein lysates ( $10 \mu \mathrm{g}$ of protein) were separated by SDS-PAGE (10\% running, 4\% stacking) and transferred to a polyvinylidene fluoride membrane (Immobilon-P, $0.45 \mu \mathrm{m}$ pore size). After blocking in 3.5\% protifar plus (Nutricia), the membranes were probed overnight at $4{ }^{\circ} \mathrm{C}$ with a primary antibody to detect TSP-2 (dilution 1:200) (BD Transduction Laboratories, \#611151), phospho-Akt (Ser473) (dilution 1:500) (Cell Signaling Technology, \#9271), total-Akt (dilution 1:1000) (Cell Signaling Technology, \#9272), and glyceraldehyde-3-phosphate dehydrogenase (GAPDH) (dilution 1:10000) (Fitzgerald, \#RDI-TRK5G4-6C5). Pending on the origin of the first antibody, membranes were incubated with a horseradish-linked polyclonal goat anti-rabbit (dilution 1:2000) (Cell Signaling Technology, \#7074) or rabbit anti-mouse antibody (dilution 1:2000) (DAKO, cat.P0161) followed by visualization of the signal using Enhanced Chemi Luminiscence. Protein band intensities were quantified using Quantity One software (Bio-Rad Laboratories Inc.) and presented relative to GAPDH protein expression.

\section{Statistical analysis}

All data are presented as mean \pm standard error of mean (SEM). Mann-Whitney U-test or unpaired $t$ test were used, when appropriate, to assess statistical significance between groups. Survival curves were obtained by the Kaplan-Meier method, and compared by the Fisher's Exact test. Probability values $<0.05$ were considered statistically significant. 


\section{Supplemental figures}

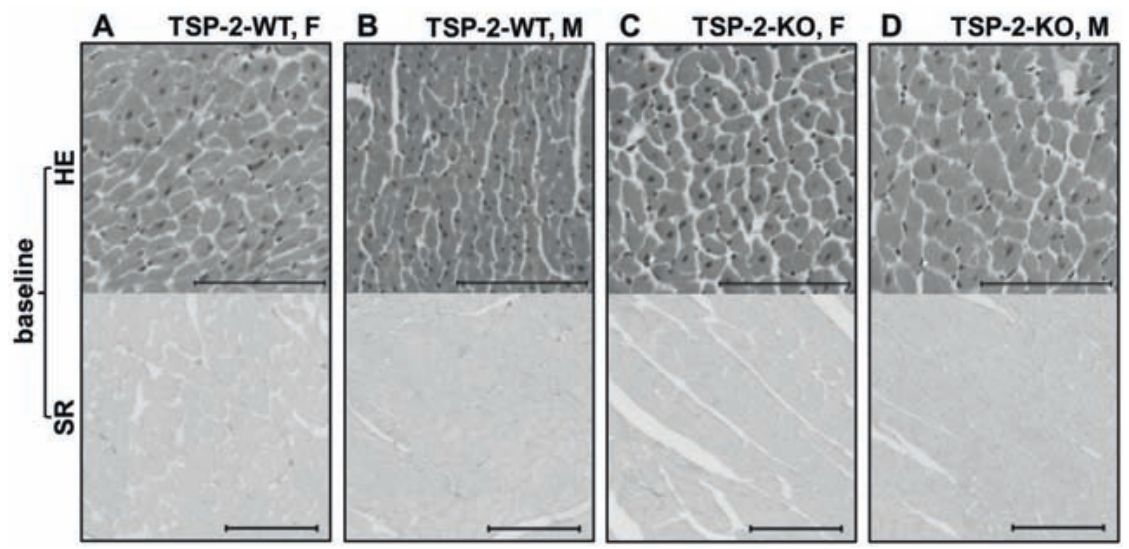

Supplemental figure 1. WT and TSP-2-KO mice had no histological abnormalities at baseline. (A-D, upper part) Histological examination on hematoxylin and eosin (HE) stained sections showed normal tissue morphology in WT and TSP-2-KO hearts at baseline. (A-D, lower part) Collagen deposition throughout the cardiac tissue was similar in WT and TSP-2-KO mice as indicated by sirius red (SR) staining. Scale bars represent $100 \mu \mathrm{m}$.
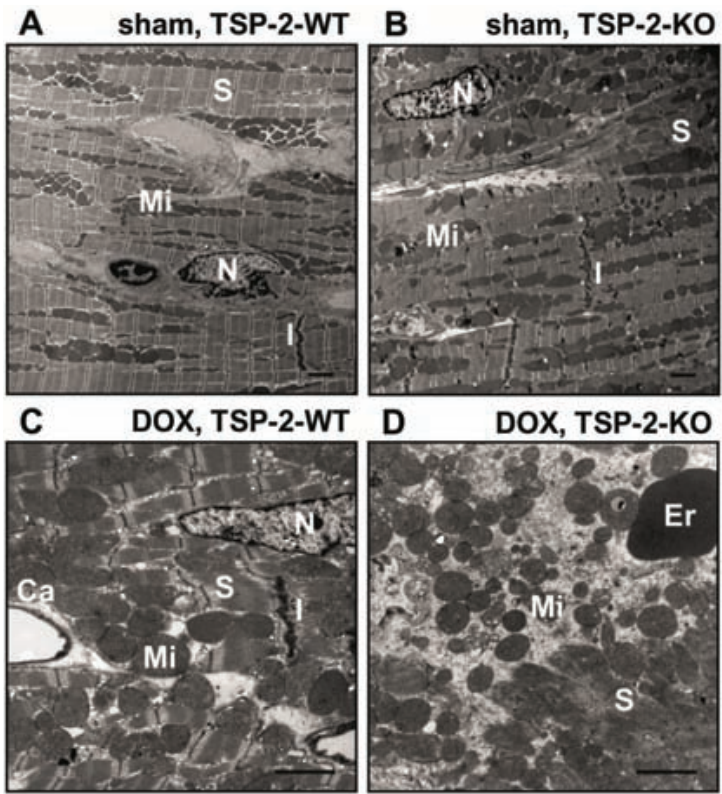

Supplemental figure 2. Increased cardiomyocyte damage in the absence of TSP-2 after DOX. Electron microscopic examination of WT and TSP-2-KO hearts after DOX and sham treatment. (A and B) TSP-2-WT and $\mathrm{KO}$ mice showed no ultrastructural abnormalities at baseline. ( $C$ and $D$ ) Mitochondrial swelling in WT and TSP-2-KO mice indicated DOX-induced myocyte stress in both genotypes. Pronounced cardiomyocyte suffering in the absence of TSP-2 was characterized by mitochondrial extraction, loss of sarcomeric structures and myolysis. Ca, capillary; Er, erythrocyte; I, intercalated disc; Mi, mitochondrion; N, nucleus; S, sarcomere. Scale bars represent $2 \mu \mathrm{m}$. 
A

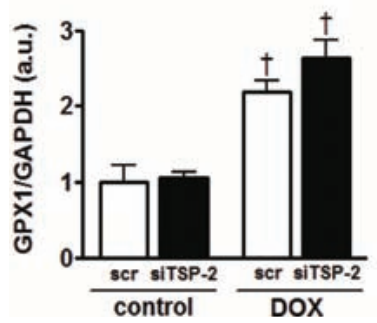

B

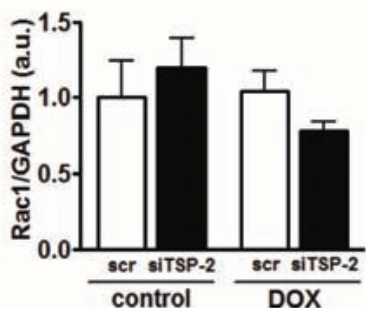

Supplemental figure 3. Induction of oxidative stress in cardiac fibroblasts in vitro, is not related to TSP-2 expression. Transcript levels of the antioxidant GPX1, and the oxidant Rac1 were determined in siTSP-2 treated cardiac fibroblasts after exposure to $1 \mu \mathrm{M}$ DOX for twenty-four hours. (A) GPX1 transcript levels were equally induced in siTSP-2- and scrambled RNA-treated cardiac fibroblasts after DOX. (B) Rac1 mRNA levels were not different between scrambled RNA- or siTSP-2-treated cardiac fibroblasts, nor did DOX had an effect on its expression. $+P<0.05$, DOX versus saline-treated cardiac fibroblasts. $S c r$ indicates scrambled SiRNA, and siTSP-2 indicates siRNA against TSP-2 of rat origin. Data are expressed as mean \pm SEM.

A

Cardiomyocyte, in vitro

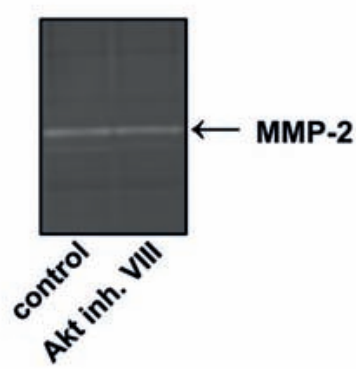

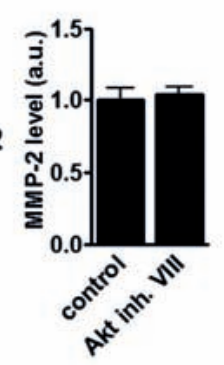

B

\section{Cardiac fibroblast, in vitro}

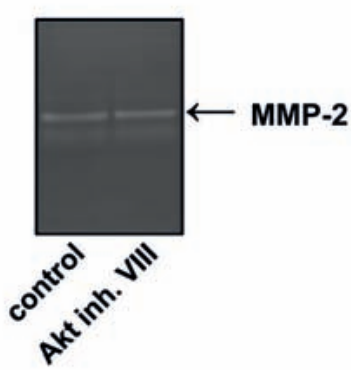

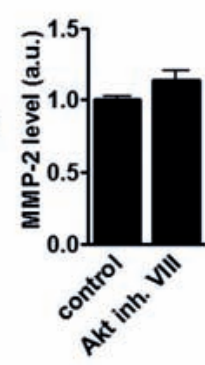

Supplemental figure 4. MMP-2 levels in cardiomyocytes and fibroblasts are unrelated to the extent of Aktphosphorylation. (A and B) Inhibition of Akt phosphorylation did not change MMP-2 activity in cardiomyocytes and cardiac fibroblasts, indicating that activation of MMP-2 is independent from the extent of Akt signaling. Data are expressed as mean $\pm \mathrm{SEM}, \mathrm{n}=4$ per group. ${ }^{*} P<0.05$, Akt inhibitor VIII-treated versus control. 


\section{Supplemental references}

1 Kyriakides TR, Leach KJ, Hoffman AS, Ratner BD, Bornstein P. Mice that lack the angiogenesis inhibitor, thrombospondin 2, mount an altered foreign body reaction characterized by increased vascularity. Proc Natl Acad Sci U S A. 1999;96(8):4449-4454.

2 Heymans S, Luttun A, Nuyens D, Theilmeier G, Creemers E, Moons L, Dyspersin GD, Cleutjens JP, Shipley M, Angellilo A, Levi M, Nube O, Baker A, Keshet E, Lupu F, Herbert JM, Smits JF, Shapiro SD, Baes M, Borgers M, Collen D, Daemen MJ, Carmeliet P. Inhibition of plasminogen activators or matrix metalloproteinases prevents cardiac rupture but impairs therapeutic angiogenesis and causes cardiac failure. Nat Med. 1999;5(10):1135-1142.

3 Heymans S, Lupu F, Terclavers S, Vanwetswinkel B, Herbert JM, Baker A, Collen D, Carmeliet P, Moons L. Loss or inhibition of UPA or MMP-9 attenuates LV remodeling and dysfunction after acute pressure overload in mice. Am J Pathol. 2005;166(1):15-25.

4 Schroen B, Heymans S, Sharma U, Blankesteijn WM, Pokharel S, Cleutjens JP, Porter JG, Evelo CT, Duisters R, van Leeuwen RE, Janssen BJ, Debets JJ, Smits JF, Daemen MJ, Crijns HJ, Bornstein P, Pinto YM. Thrombospondin-2 is essential for myocardial matrix integrity: increased expression identifies failureprone cardiac hypertrophy. Circ Res. 2004;95(5):515-522.

5 De Windt LJ, Willemsen PH, Popping S, Van der Vusse GJ, Reneman RS, Van Bilsen M. Cloning and cellular distribution of a group II phospholipase A2 expressed in the heart. J Mol Cell Cardiol. 1997;29(8):2095-2106. 



\section{Chapter 5}

\section{MicroRNA-18 and -19 regulate CTGF and TSP-1 expression in age-related heart failure}

Geert C. van Almen, Wouter Verhesen, Rick E.W. van Leeuwen, Mathijs van de Vrie, Casper Eurlings, Mark W.M. Schellings, Melissa Swinnen, Jack P.M. Cleutjens, Marc A.M.J. van Zandvoort, Stephane Heymans, Blanche Schroen 


\begin{abstract}
To understand the process of cardiac aging, it is of crucial importance to gain insight in the age-related changes in gene expression in the senescent failing heart. Age-related cardiac remodeling is known to be accompanied by changes in extracellular matrix (ECM) gene and protein levels. Small non-coding microRNAs regulate gene expression in cardiac development and disease, and have been implicated in the aging process and in the regulation of ECM proteins. However, their role in age-related cardiac remodeling and heart failure is unknown. In the present study, we investigated the aging-associated microRNA cluster 17 92, which targets the ECM proteins connective tissue growth factor (CTGF) and thrombospondin-1 (TSP-1). We employed aged mice with a failure resistant (C57BI6) and failure prone (C57BI6×129Sv) genetic background and extrapolated our findings to human age-associated heart failure. In agingassociated heart failure, we linked an aging-induced increase in the ECM proteins (CTGF) and (TSP-1) to a decreased expression of their targeting microRNAs $-18 \mathrm{a},-19 \mathrm{a}$ and $-19 b$, all members of the miR-17 92 cluster. Failure resistant mice showed an opposite expression pattern for both the ECM proteins and the microRNAs. We showed that these expression changes are specific for cardiomyocytes and are absent in cardiac fibroblasts. In cardiomyocytes, modulation of miR-18/19 changes the levels of ECM proteins CTGF, TSP-1 and collagens type 1 and 3. Together, our data support a role for cardiomyocyte-derived miR-18/19 during cardiac aging, in the fine-tuning of cardiac ECM protein levels. During aging, decreased miR-18/19 and increased CTGF and TSP-1 levels identify the failure-prone heart.
\end{abstract}




\section{Introduction}

Aging is considered a multifactorial process that is controlled by genetic and environmental factors and ultimately leads to deterioration of body and organ functions. Heart failure (HF) is a typical age-associated disease and is characterized by unique physiological and morphological changes in aged myocardium (reviewed in ${ }^{1}$ ). Although the rate and mechanism through which an animal or tissue ages differs amongst species, the constant remodeling and accumulation of the extracellular matrix (ECM) is recognized as a key feature of cardiac aging in humans, mice and rats and contributes to the structural changes that lead to HF with advancing age. ${ }^{2-4}$ In particular, the expression of the ECM proteins SPARC ${ }^{5}$, fibronectin ${ }^{6}$, thrombospondin-2 (TSP- 2$)^{7}$, and connective tissue growth factor $(\mathrm{CTGF})^{8}$ increases with aging. Both TSP-2 knockout mice and cardiomyocyte specific CTGF transgenic animals develop spontaneous agerelated cardiomyopathy ${ }^{7,9}$, confirming a role of ECM proteins in age-related cardiac remodeling. However, the underlying mechanisms that drive the age-related gene expression of ECM molecules remain elusive.

The identification of small non-coding microRNAs (miRNAs) opened new doors for investigating the regulation of gene expression by adding another layer of control at the post-transcriptional level. MiRNAs are 22 nucleotides long RNA molecules that can target mRNAs for translational repression or degradation by complementary binding to specific sequences in the protein-coding gene transcript. The first implication of miRNAs in aging was provided by a study that showed that lin-4 and its target protein lin-14 determine longevity in $C$. elegans by affecting the insulin-like signaling pathway. ${ }^{10}$ Now, miRNAs have emerged as important mediators of diverse age-associated pathologies, from cancer and diabetes to neurodegenerative disorders ${ }^{11}$, and increasing evidence demonstrates altered miRNA expression profiles in aging muscle, brain, lung, and liver (reviewed $\mathrm{in}^{12}$ ). In addition, miRNAs are regulated during cellular senescence, and complete loss of miRNA function caused premature senescence in embryonic fibroblasts ${ }^{13}$, putting miRNAs in the forefront of cellular senescence in different organs and cell types. ${ }^{11,12}$ Their role in the heart was first suggested by expression profiling studies showing changes in the expression of specific miRNAs in failing human hearts. ${ }^{14,15}$ Further animal models proved the involvement of specific miRNAs in cardiac development, function, and under pathological conditions. ${ }^{16-18}$ Nevertheless, despite their proven importance in aging, cardiac disease and development, a role for miRNAs during cardiac aging and age-related $\mathrm{HF}$ remains to be elucidated.

The present study investigated the role of the miR-17 92 cluster in the aged heart, in view of its central role in regulation of matrix genes and in cellular aging. ${ }^{19-21}$ This cluster encodes six miRNAs (miR-17, miR-18a, miR-19a, miR-19b, miR-20a, and miR92a-1) which are located within an 800 base-pair region of human chromosome 13. Originally, the miR-17 92 cluster was linked to tumor genesis, and transcription of the cluster was found to be directly activated by the proto-oncogene c-Myc ${ }^{22}$ (reviewed $\mathrm{in}^{23}$ ). The pro-oncogenic activity of miR-17 92 partially involves the regulation of the 
ECM proteins CTGF and thrombospondin-1 (TSP-1) by the cluster members miR-18 and miR-19, through sequence-specific targeting within the $3^{\prime}$-untranslated region (3'-UTR) of these gene transcripts (Supplemental figure 1). ${ }^{19}$ Interestingly, cardiogenesis was severely hampered in mice deficient for miR-17 92, suggesting an important role for this cluster in cardiac development. ${ }^{24}$ This, together with miR-18 and miR-19 targeting CTGF and TSP-1 and the fact that ECM proteins are crucial for healthy cardiac aging, has led us to hypothesize that these miRNAs play a role in age-related cardiac remodeling. Therefore, we investigated whether age-related changes in miR-18a, miR-19a, and miR-19b expression regulate CTGF, TSP-1 and collagen levels in rodent models of aging-associated heart failure and in the human failing heart.

\section{Methods}

An expanded methods section is provided in the Supplemental material (pag. 121).

\section{Mice}

Male C57BI6 mice were obtained from Janvier (Le Genest Saint Isle, France) and mice on a C57BI6×129Sv genetic background were generated within the animal facility of the University of Maastricht. All animals were housed under standard day-night rhythm and ad libitum conditions until 12, 52 and 104 weeks of age. Cardiac function was assessed under sedation ( $2 \%$ isoflurane), followed by removal of the hearts for further histological and molecular analyses. For histology, hearts were fixed in $1 \%$ paraformaldehyde, embedded in paraffin and stained with Sirius red. For high magnification analysis, hearts were fixed in $2.5 \%$ glutaraldehyde. Electron microscopic images were made with a Philips CM100 (F.E.I., Eindhoven, The Netherlands).

This study was approved by the Institutional Animal Research Committee and conforms with the guidelines for the use of laboratory animals formulated in the Dutch law on care and use of experimental animals.

\section{Patients}

Nineteen subjects diagnosed with idiopathic cardiomyopathy (ICM) were included based on age and cardiac function. Five old patients (60.5 years \pm 0.8 ) with preserved cardiac function (ejection fraction between 40 and 55 percent) and no signs of cornary artery disease (ejection fraction (EF): $45.6 \% \pm 9.2$ ), and nine ICM patients at older age (67.0 years \pm 4.3 ) with a severely compromised cardiac function (EF: $18.9 \% \pm 3.0$ ) were compared to a group of five young patients (33.2 years \pm 4.4 ) with preserved cardiac function (EF: $46.5 \% \pm 4.4$ ). This study occurred in line with the recommendations of the institutional ethics committee of the University Hospital Maastricht. 


\section{In vitro experiments}

Neonatal rat cardiomyocytes (NRCMs) and cardiac fibroblasts (NRCFs) were isolated from 1- to 3-day-old Lewis rats as described previously. ${ }^{25}$

For aging of cardiomyocytes in vitro, NRCMs were cultured for 4 and 21 days, and autofluorescent lipofuscin was excited using a laser at $488 \mathrm{~nm}$, with confocal detection at 510-560 nm (Nikon Eclipse E600FN, Tokyo, Japan). Hoechst-stained nuclei were excited with a two-photon Spectra-Physics Tsunami laser (Spectra-Physics, Irvine, CA, USA) centered at $800 \mathrm{~nm}$ and visualized at $400-480 \mathrm{~nm}$. The lipofuscin content per cardiomyocyte was determined using Leica Qwin image processing and analysis software (Leica. Microsystems Cambridge Ltd, Cambridge, UK).

For the overexpression or inhibition of miR-18a and miR-19b, NRCMs and NRCFs were transfected with $80 \mathrm{~nm}$ miRIDIAN hairpin inhibitor (antagomiR) miR-18a (\#IH300487-06) or miR-19b (\#IH-300489-05), or with miRIDIAN mimic miR-18a (\#C300487-05), or miR-19b (\#C-300489-03) (Dharmacon, Colorado, CO, USA). Cells transfected with miRIDIAN microRNA hairpin inhibitor negative control \#1 (\#IN-001005-01), and miRIDIAN microRNA mimic negative control \#2 (\#CN-002000-01) served as control groups.

\section{RNA isolation and Real-Time PCR}

Total RNA was extracted from homogenized cells and heart tissues using the mirVANA miRNA isolation kit (Ambion, Austin, TX, USA) according to the manufacturer's protocol. Gene transcript levels of CTGF, TSP-1, collagen 1A1 and collagen 3A1 (primer sequences are listed in supplemental table 4) or miRNA expression were detected with the MyIQ Single Color Real-Time PCR detection System (Bio-Rad, Hercules, CA, USA). MiR-17, miR18a, miR-19a, miR-19b, miR-20, and miR-92a-1 expression was measured using miRNA-specific miScript Primer Assays (Qiagen, Hilden, Germany). All expression levels were presented relative to glyceraldehydes-3-phosphate dehydrogenase (GAPDH).

\section{Immunoblotting}

Proteins were extracted from left ventricular heart tissue, and in vitro from NRCM and NRCFs. Protein lysates were separated by SDS-PAGE, and transferred to a polyvinylidene fluoride membrane (Immobilon-P, $0.45 \mu \mathrm{m}$ pore size). The membranes were probed overnight at $4^{\circ} \mathrm{C}$ with a primary antibody to detect CTGF (GeneTex Inc., Irvine, CA, USA; \#GTX26992), TSP-1 (in-house rabbit anti-human TSP-1 was kindly provided by MF Hoylaerts ${ }^{26}$, University of Leuven, Belgium), and glyceraldehyde-3-phosphate dehydrogenase (GAPDH) (Fitzgerald Inc., Concord, MA, USA; \#RDI-TRK5G4-6C5). Protein levels were determined using Quantity One software (Bio-Rad Laboratories Inc.) and presented relative to GAPDH protein expression. 


\section{In Situ Hybridization}

Mouse left ventricular heart tissue was used for miRNA in situ hybridization as described previously. ${ }^{27}$ Double DIG-labeled Locked Nucleic Acid (LNA) hybridization probes complementary to mouse mature miR-18a (5DIGN/CTATCTGCACTAGATGCACCTTA/3DIG_N) (\#38462-15), miR-19b (5DIGN/TCAGTTTTGCATGGATTTGCACA/3DIG_N) (\#38092-15), and a scrambled probe (5DIGN/GTGTAACACGTCTATACGCCCA/3DIG_N) (\#99004-15) were purchased from Exiqon (Vedbaek, Denmark).

\section{Statistical analysis}

All data are expressed as mean \pm standard error of the mean (SEM). Differences between groups were evaluated by Student's $t$-test or one-way ANOVA with Bonferroni post-hoc test when appropriate. Differences in interstitial and perivascular fibrosis were analyzed by two-way ANOVA and Bonferroni post-hoc test. Probability values $<0.05$ were considered statistically significant.

\section{Results}

\section{HF prone mice develop cardiac dilation and dysfunction at old age}

As a model for age-related $\mathrm{HF}$ in rodents, we examined age-associated changes in cardiac function in two genetically different mouse strains; i.e. C57BI6 (HF resistant) and C57BI6×129Sv (HF prone). Both strains developed normally and echocardiographic analysis showed no differences in cardiac function at 12 and 52 weeks of age. However, fractional shortening was significantly compromised in 104 weeks old HF prone mice compared to age-matched HF resistant hearts (Table 1). Indices of left ventricular wall thickness, LVPW and IVS, indicated progressive thinning of the LV wall with aging in HF prone mice, followed by ventricular dilation at old age (Table 1). HF resistant mice on the other hand had no ventricular dilation and showed a tendency to increased wall thickness with aging, resulting in a preserved cardiac function at old age (Table 1). Age-associated cardiac dysfunction in HF prone mice was further shown by significantly higher heart, kidney and lung weights at old age (Table 1). Thus, poor cardiac aging in HF prone mice was characterized by a dilated cardiomyopathy-like phenotype as is seen in human HF. 
Table 1. Echocardiographic and morphometric analysis of male mice at different ages

\begin{tabular}{lcccccc}
\hline & & HF resistant & \multicolumn{3}{c}{ HF prone } \\
\hline & 12 weeks & 52 weeks & 104 weeks & 12 weeks & 52 weeks & 104 weeks \\
& $\mathrm{n}=8$ & $\mathrm{n}=8$ & $\mathrm{n}=9$ & $\mathrm{n}=9$ & $\mathrm{n}=7$ & $\mathrm{n}=8$ \\
\hline FS (\%) & $28 \pm 1.5$ & $29 \pm 1.6$ & $29 \pm 1.7$ & $28 \pm 2.0$ & $29 \pm 3.5$ & $20 \pm 2.2^{*+\ddagger}$ \\
LVIDd (mm) & $4.0 \pm 0.1$ & $4.2 \pm 0.1$ & $4.2 \pm 0.1$ & $3.8 \pm 0.2$ & $3.7 \pm 0.2$ & $4.6 \pm 0.2^{*+}$ \\
LVIDs (mm) & $2.9 \pm 0.1$ & $3.0 \pm 0.1$ & $3.0 \pm 0.1$ & $2.7 \pm 0.1$ & $2.7 \pm 0.2$ & $3.7 \pm 0.3^{*+\ddagger}$ \\
LVPWd (mm) & $0.75 \pm 0.03$ & $0.84 \pm 0.03^{*}$ & $0.90 \pm 0.03^{*}$ & $0.99 \pm 0.11$ & $0.91 \pm 0.05$ & $0.88 \pm 0.06$ \\
LVPWs (mm) & $1.16 \pm 0.06$ & $1.18 \pm 0.06$ & $1.27 \pm 0.04$ & $1.40 \pm 0.08^{\ddagger}$ & $1.22 \pm 0.04$ & $1.02 \pm 0.07^{*+\ddagger}$ \\
IVSd (mm) & $0.72 \pm 0.01$ & $0.81 \pm 0.03^{*}$ & $0.81 \pm 0.04^{*}$ & $0.96 \pm 0.15$ & $0.91 \pm 0.05$ & $0.83 \pm 0.03$ \\
IVSs (mm) & $1.03 \pm 0.01$ & $1.22 \pm 0.04^{*}$ & $1.18 \pm 0.07$ & $1.41 \pm 0.18$ & $1.37 \pm 0.09$ & $1.06 \pm 0.06^{\dagger}$ \\
Heart rate (bpm) & $482 \pm 14$ & $473 \pm 11$ & $493 \pm 8$ & $506 \pm 15$ & $521 \pm 9^{\ddagger}$ & $502 \pm 25$ \\
Body weight (g) & $25.0 \pm 0.3$ & $30.8 \pm 0.5^{*}$ & $33.2 \pm 0.8^{*+}$ & $23.5 \pm 1.1$ & $29.8 \pm 0.7^{*}$ & $29.8 \pm 0.7^{* \ddagger}$ \\
HW/BW ratio (mg/g) & $4.8 \pm 0.1$ & $4.8 \pm 0.2$ & $4.4 \pm 0.1^{*}$ & $5.4 \pm 0.2^{\ddagger}$ & $5.5 \pm 0.1^{\ddagger}$ & $6.0 \pm 0.3^{\ddagger}$ \\
LuW/BW ratio (mg/g) & $5.7 \pm 0.3$ & $5.7 \pm 0.2$ & $6.2 \pm 0.2$ & $5.9 \pm 0.3$ & $5.9 \pm 0.3$ & $7.5 \pm 1.0$ \\
LiW/BW ratio (mg/g) & $48.0 \pm 0.7$ & $47.7 \pm 1.1$ & $48.1 \pm 0.9$ & $47.0 \pm 2.8$ & $46.6 \pm 1.6$ & $53.7 \pm 3.8$ \\
KiW/BW ratio(mg/g) & $8.0 \pm 0.2$ & $8.7 \pm 0.3$ & $8.7 \pm 0.2$ & $8.8 \pm 0.4$ & $11.9 \pm 0.3^{* \ddagger}$ & $11.3 \pm 0.4^{* \ddagger}$ \\
\hline
\end{tabular}

FS, fractional shortening; LVIDd and LVIDs, left ventricular internal diameter in diastole and systole; LVPWd and LVPWs, left ventricular posterior wall thickness in diastole and systole; IVSd and IVSs, intraventricular septum thickness in diastole and systole; HW, heart weight; BW, body weight; LuW, lung weight; LiW, liver weight; KiW, kidney weight. ${ }^{*} P \leq 0.05$ versus 12 weeks of age with the same genotype; $+P \leq 0.05$ versus 52 weeks of age with the same genotype; $¥ P \leq 0.05$ versus $H F$ resistant mice with the same age.

HF prone hearts have more interstitial fibrosis and increased levels of CTGF and TSP-1

Dilated cardiomyopathy is characterized by increased deposition of interstitial collagen, stiffening the heart and compromising its contractility. ${ }^{6,28}$ Histological examination of cardiac sections showed significant interstitial fibrosis in $\mathrm{HF}$ prone mice as compared to 52 weeks littermates and to 104 weeks old HF resistant hearts (figure $1 \mathrm{~A}$ and $B)$. On the other hand, the mild increase of collagen in aged HF resistant mice was not significant (Figure $1 \mathrm{~A}$ and $\mathrm{B}$ ), while accumulation of interstitial collagen was similar in 12 and 52 weeks old $\mathrm{HF}$ resistant and HF prone mice. As expected, perivascular fibrosis increased with aging, but was not different between HF prone and HF resistant hearts (Figure $1 \mathrm{~A}$ and $\mathrm{B}$ ).

The ECM proteins CTGF and TSP-1 are recognized as powerful regulators of ECM remodeling and are mediators of tissue fibrosis in humans, mice and rats. ${ }^{29,}{ }^{30} \mathrm{We}$ found significantly increased cardiac transcript and protein levels of CTGF and TSP-1 in 104 weeks old HF prone mice (Figure 2A and B; Supplemental figure 2). Vice versa, the hearts of aged HF resistant mice had no induction of CTGF and TSP-1 transcript or protein levels (Figure $2 A$ and $B$ ). Therefore, increased interstitial fibrosis and CTGF and TSP-1 expression characterize the HF prone aged heart. 
A

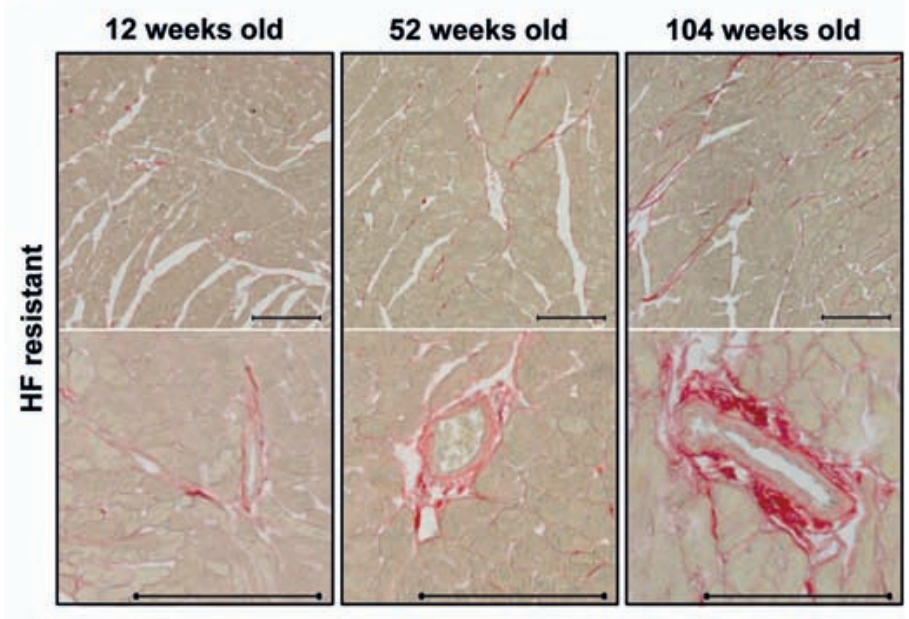

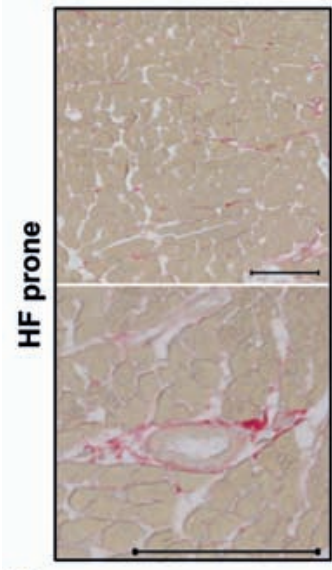

B

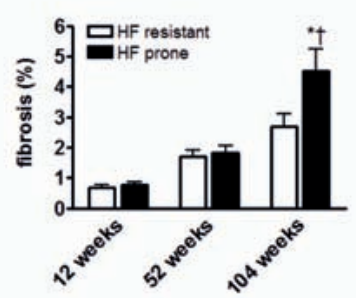

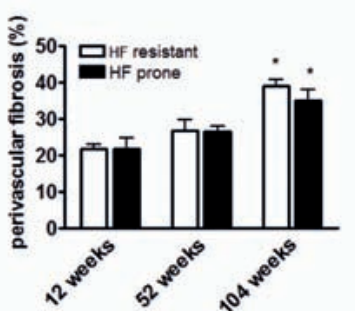

Figure 1. Enhanced left ventricular interstitial fibrosis in old HF prone mice. (A) Histological analysis of the hearts of HF resistant and HF prone mice by Sirius Red staining. Photos show representative areas of interstitial fibrosis (upper panel) and collagen deposition in de perivascular area (lower panel). Scale bars represent $100 \mu \mathrm{m}$. (B) Quantitive analysis of the interstitial and perivascular collagen content in HF resistant (12 weeks, n=8; 52 weeks, $n=8$; and 104 weeks, $n=9$ ) and HF prone mice ( 12 weeks, n=6; 52 weeks, $n=11$; and 104 weeks, $n=9$ ) revealed increased interstitial fibrosis in the left ventricles of 104 weeks old HF prone mice. Perivascular fibrosis was significantly increased in 104 weeks old hearts, but was not different between $\mathrm{HF}$ resistant and $\mathrm{HF}$ prone mice. Data are presented as mean $\pm \mathrm{SEM}$. ${ }^{*} P \leq 0.05$ versus 52 weeks old $\mathrm{HF}$ prone mice; $+P \leq 0.05$ versus 104 weeks old $\mathrm{HF}$ resistant mice. 
A

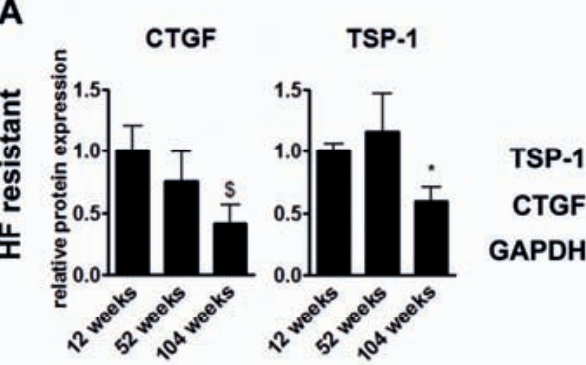

B

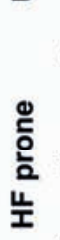

TSP-1

TSP-1

CTGF

GAPDH
CTGF

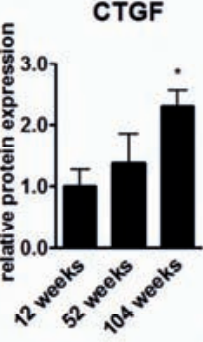

C

miR-18a

miR-19a

miR-19b
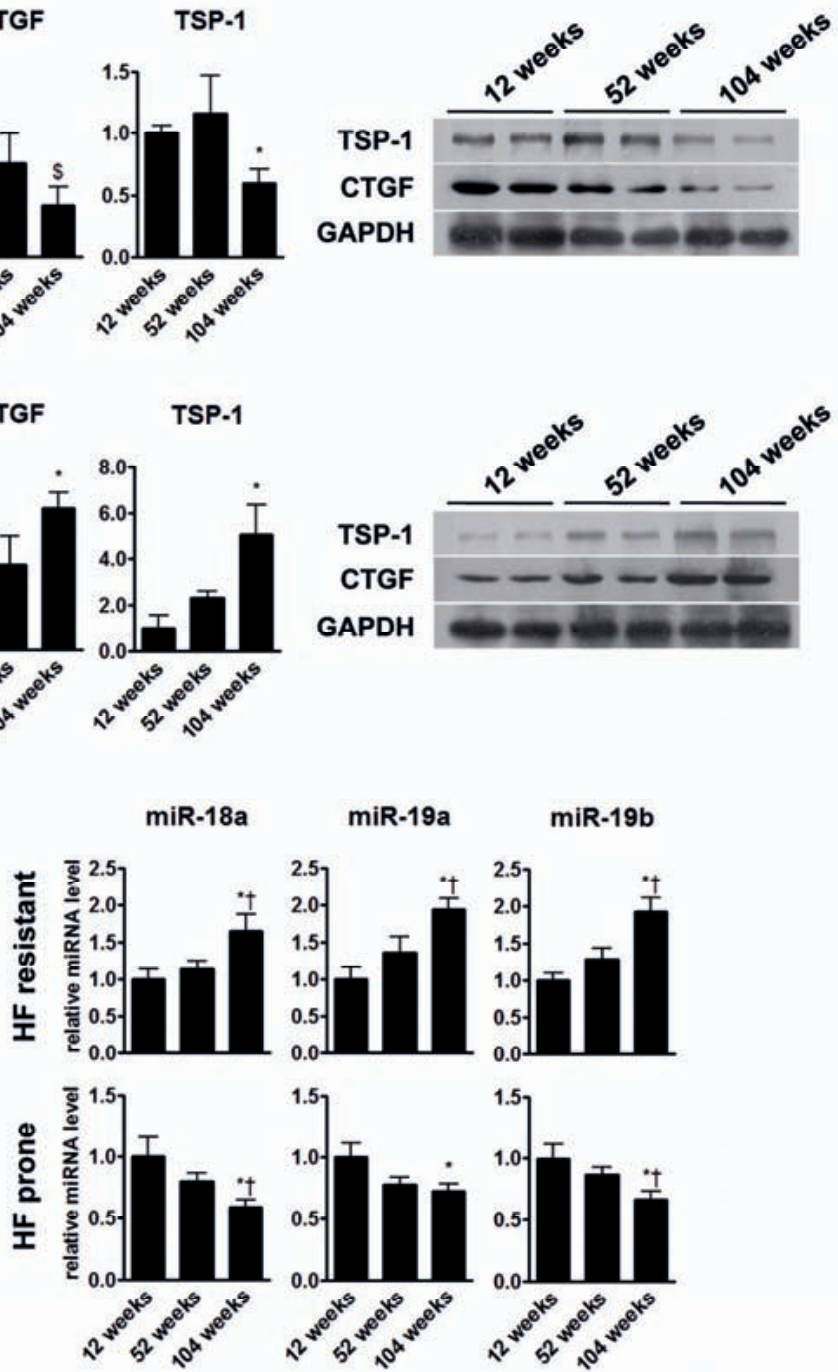

Figure 2. Opposite expression profiles in HF resistant versus HF prone mice. CTGF, TSP-1, miR-18a, miR19a and miR-19b levels in aged HF resistant (12 weeks, $n=8 ; 52$ weeks, $n=8$; and 104 weeks, $n=9$ ) and HF prone mice ( 12 weeks, $n=6 ; 52$ weeks, $n=11$; and 104 weeks, $n=9$ ). ( $A$ and $B$ ) Immublotting was performed on four mice per age group and revealed significant induction of CTGF and TSP-1 protein expression in failing hearts of 104 weeks old HF prone mice, whereas CTGF and TSP-1 levels were reduced in old HF resistant mice. Immunoblots show representative protein bands of TSP-1, CTGF and GAPDH (C) RT-PCR analysis showed increased expression of miR-18a, miR-19a, and miR-19b in 104 weeks old HF resistant hearts, whereas age-matched $\mathrm{HF}$ prone mice had decreased expressions. miRNA expression and CTGF and TSP-1 protein levels were normalized for GAPDH expression and presented as mean \pm SEM. ${ }^{*} P \leq 0.05$ versus 12 weeks of age; $+P=0.05$ versus 12 weeks of age. 
Opposite cardiac miR-17 92 cluster expression profiles in HF resistant and HF prone aging

CTGF and TSP-1 have been identified as target genes of the miR-17 92 cluster $^{19}$, more specifically of the cluster members miR-18a and miR-19a/b. ${ }^{21,}{ }^{31}$ We found opposite expression profiles of the miR-17 92 cluster in HF prone aging compared to aging with preserved cardiac function. At 104 weeks of age, HF prone mice had significantly reduced expression levels of miR-17, miR-18a, miR-19a, miR-19b, miR-20a, and miR-92a1 as compared to 12 weeks littermates (Figure 2C and Supplemental table 1), coinciding with the observed increased presence of their targets TSP-1 and CTGF. Aging of HF resistant mice, on the other hand, was accompanied by significantly enhanced expression of these miRNAs, except for miR-17 and miR-20a (Supplemental table 1).

\section{The miR-18/19 - CTGF/TSP-1 axis is regulated in human age-associated heart failure}

The three miR-17 92 cluster members miR-18a and miR-19a and miR-19b specifically target the ECM proteins CTGF and TSP-1. To investigate the role of these genes in human HF, we studied their expression profiles in cardiac biopsies of idiopathic cardiomyopathy (ICM) patients at old age with a moderately decreased or preserved systolic function (ejection fraction (EF) between 40 and 55 percent) ${ }^{32}$ and severely impaired cardiac function (EF < 30 percent), and compared them to young ICM subjects. In line with reduced expression levels in failing hearts of old mice, decreased miR-18a, miR19a and miR-19b expression was associated with severe heart failure at old age (Figure $3 A$ ), while miRNA expression in old patients with a preserved function was not different from young ICM patients (Figure 3A). Additionally, CTGF and TSP-1 transcript levels were significantly induced in old failing ICM hearts, which further corroborates our findings in old HF prone mice (Figure $3 \mathrm{~B}$ ). Together these data suggest that regulation of CTGF and TSP-1 is the result of the shared expression of miR-18a, miR-19a, and miR19b, enabling modest changes in miRNA expression to control transcriptional repression. 

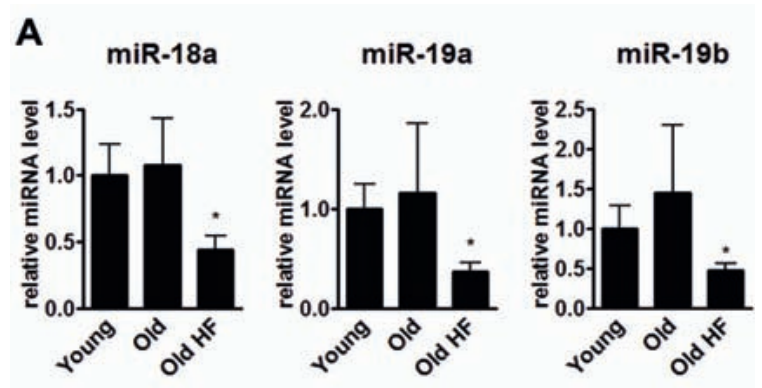

B

CTGF

TSP-1
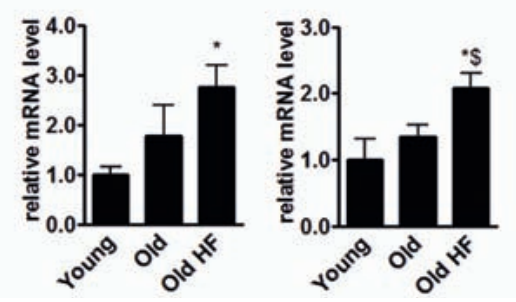

Figure 3. CTGF and TSP-1 expression are elevated in human HF. RT-PCR analysis of miR-18a, miR-19a, miR19b, CTGF and TSP-1 transcript levels in myocardial biopsies from ICM patients at older age with normal $(n=5)$ and severely impaired $(n=9)$ cardiac function. Transcript levels were compared to the expression in young ICM subjects with a preserved cardiac function $(n=5)(A)$ MiR-18a, miR-19a and miR-19b expression were significantly decreased in older ICM patients with HF. (B) CTGF and TSP-1 transcript levels were significantly induced in older patients with a compromised cardiac function, when compared to younger ICM subjects. All data were normalized for GAPDH expression and presented as mean \pm SEM. ${ }^{*} P \leq 0.05$ versus young ICM patients. $\$ P=0.06$ in failing versus non-failing hearts of older ICM patients.

The miR-18/19 - CTGF/TSP-1 axis is regulated in aged cardiomyocytes in vitro

To gain further insight in the role of the miR-17 92 cluster in aging of cardiomyocytes, neonatal rat cardiomyocytes (NRCMs) were aged in vitro, and miRNA levels were determined.

Aging of cardiomyocytes in vitro was validated by comparing the lipofuscin content in 4 and 21 days old NRCMs to the intracellular accumulation of lipofuscin in the hearts of 12 and 104 weeks old C57BI6 mice. Lipofuscin, being autofluorescent, nondegradable biological "garbage", is a hallmark of aging in post-mitotic cells such as cardiomyocytes and neurons. ${ }^{33}$ On high magnification examination using electron microscopy, accumulation of lipofuscin was rarely observed in the hearts of 12 weeks old mice, whereas a large amount of intralysosomal lipofuscin inclusions was detected in 104 weeks old mice (Figure 4A). Prolonged culturing of NRCMs resembles the aging process in mice, as lipofuscin was hardly detected at 4 days but had accumulated at 21 days, while the characteristic sarcomere structures were maintained (Figure 4A). Using optical imaging lipofuscin content per cardiomyocyte increased $>150$-fold after 21 days compared to 4 days old NRCMs (Figure 4B). 
A

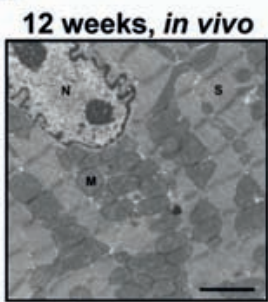

4 days, in vitro

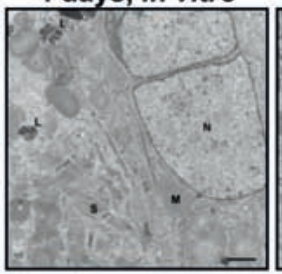

C

COL1A1
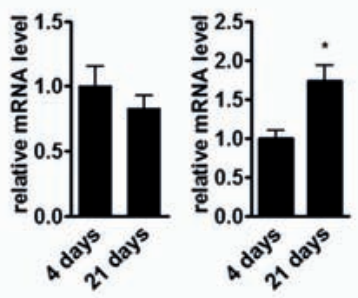

COL3A1
104 weeks, in vivo

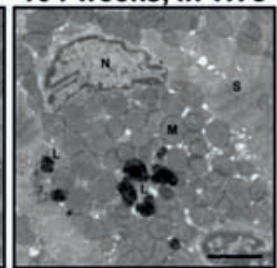

21 days, in vitro

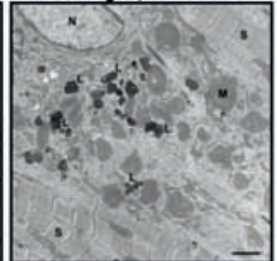

\section{E}
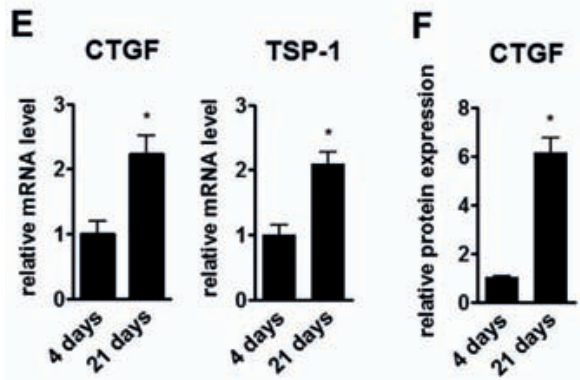

B
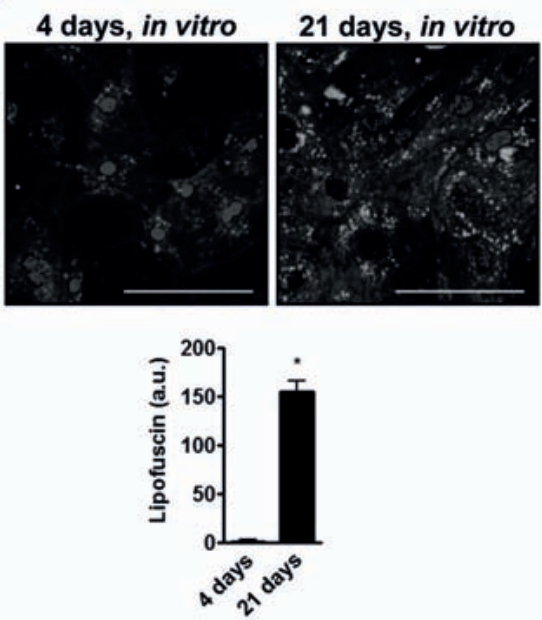

D

miR-18a miR-19a miR-19b
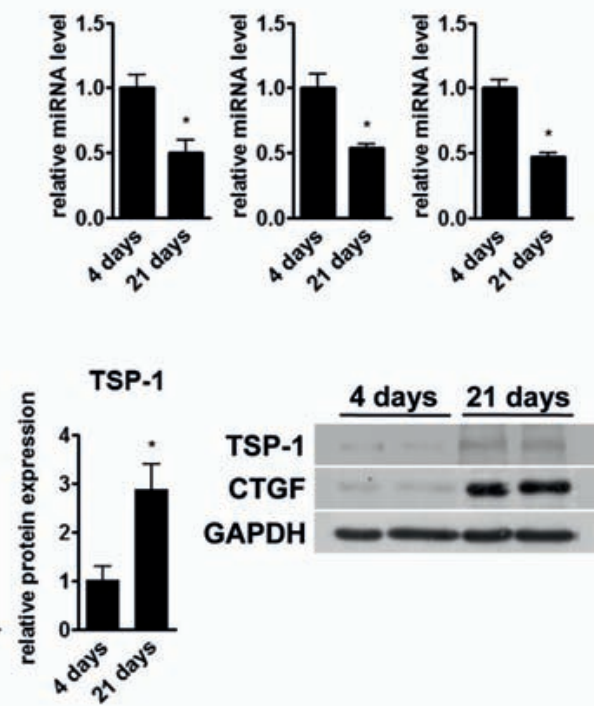

Figure 4. Aging-induced expression profiles in cardiomyocytes in vitro. (A) Electron microscopic images of the left ventricle of 12 and 104 weeks old mice, and 4 and 21 days old NRCMs showing perinuclear accumulation of lipofuscin in cardiomyocytes of 104 weeks old mice and NRCMs after 21 days in culture. Scale bars represent $2 \mu \mathrm{m}$. (B) Two-foton/confocal images and quantification of autofluorescent lipofuscin granules (perinuclear) in cultured cardiomyocytes. Nuclei are stained with Hoechst. Scale bars represent $100 \mu \mathrm{m}$ (D) RT-PCR analysis showed significant induction of collagen type 3A1 (COL3A1), but not collagen type 1A1 (COL1A1) in cultured NRCMs (D) RT-PCR analysis revealed decreased miR-18a, miR-19a, and miR-19b expression in aged NRCMs after 21 days in culture. (E) CTGF and TSP-1 transcript levels increased with NRCM aging in vitro. (F) Immunoblotting confirmed the increase CTGF and TSP-1 protein induction during cardiomyocyte aging. All in vitro experiments were performed with $n=3$ per group and protein and transcript levels were normalized for GAPDH expression. Data were presented as mean \pm SEM. $* P \leq 0.05$ versus 4 days old NRCMs. 
Beside lipofuscin accumulation, the aged myocardium is hallmarked by increased matrix deposition. To study the production of collagen in aged cardiomyocytes we determined collagen type $1 A 1$ and $3 A 1$ levels. RT-PCR analysis showed significant induction of the thin collagen type $3 A 1$, but not the thicker type $1 A 1$, in 21 days old NRCMs (Figure $4 \mathrm{C}$ ), further strengthening the aged phenotype of these cells. Taken together, prolonged culturing of NRCMs resembles two common characteristics of cardiac aging in vivo, and was therefore used to study age-related changes in cardiomyocytes in vitro.

RT-PCR analysis showed that the expression levels of all members of the miR17 92 cluster were reduced in aged cardiomyocytes, except miR-92a-1 (Figure 4D and Supplemental table 2). Importantly, miR-18a, miR-19a, and miR-19b were among the most strongly repressed miRNAs. Along with reduced expression of these miRNAs, CTGF and TSP-1 transcript and protein levels were significantly induced in aged cardiomyocytes (Figure 4E-F). These findings are conform the expression profiles in aged HF prone mice and again suggest that miR-18a, miR-19a, and miR-19b could transcriptionally repress CTGF and TSP-1 levels in cardiomyocyte aging and HF at old age.

\section{Cardiomyocyte CTGF, TSP-1 and collagen production are regulated by miR-18/19}

To investigate the cardiac localization of miR-18 and -19, we performed in situ hybridization. MiR-18a and miR-19b are abundantly expressed in the adult mouse heart, and are predominantly localized in the perinuclear area of cardiomyocytes (Figure 5A-C). This was corroborated by the finding that miR-18a and miR-19b expression was higher in cardiomyocytes compared to cardiac fibroblasts (Figure 5D). Importantly, the abundant expression of miR-18a and miR-19b in cardiomyocytes coincides with low levels of CTGF and TSP-1, whereas in cardiac fibroblasts relatively low levels of miR-18a and miR-19b were associated with high CTGF and TSP-1 transcription (Figure 5E).

Next, we performed a series of functional studies to determine the role of miR-18a and $\mathrm{miR}-19 \mathrm{~b}$ in the regulation of CTGF and TSP-1 and collagen production in cardiomyocytes and cardiac fibroblasts. Overexpression of miR-18a and miR-19b, using miRNA mimics, resulted in significant repression of CTGF and TSP-1 MRNA and protein expression in cardiomyocytes (Figure $5 \mathrm{~F}$ and $\mathrm{G}$; Supplemental figure $2 \mathrm{~A}$ ). Vice versa, blunting of miR-18a and miR-19b using antagomirs was sufficient to increase CTGF and TSP-1 transcript and protein levels in cardiomyocytes (Figure 5F and G; Supplemental figure 2A). Cardiac fibroblasts demonstrated decreased CTGF and TSP-1 transcript levels upon introduction of miR-18a and miR-19b, however, this did not result in reduced protein levels (Figure $5 \mathrm{H}$ and I). Along the same line, endogenous miRNA inhibition was not sufficient to enhance CTGF and TSP-1 transcript and protein expression in cardiac fibroblasts (Figure $5 \mathrm{H}$ and I; Supplemental figure 2). These results show that regulation of CTGF and TSP-1 by miR-18a and miR-19b is uniquely restricted to the cardiomyocyte. 

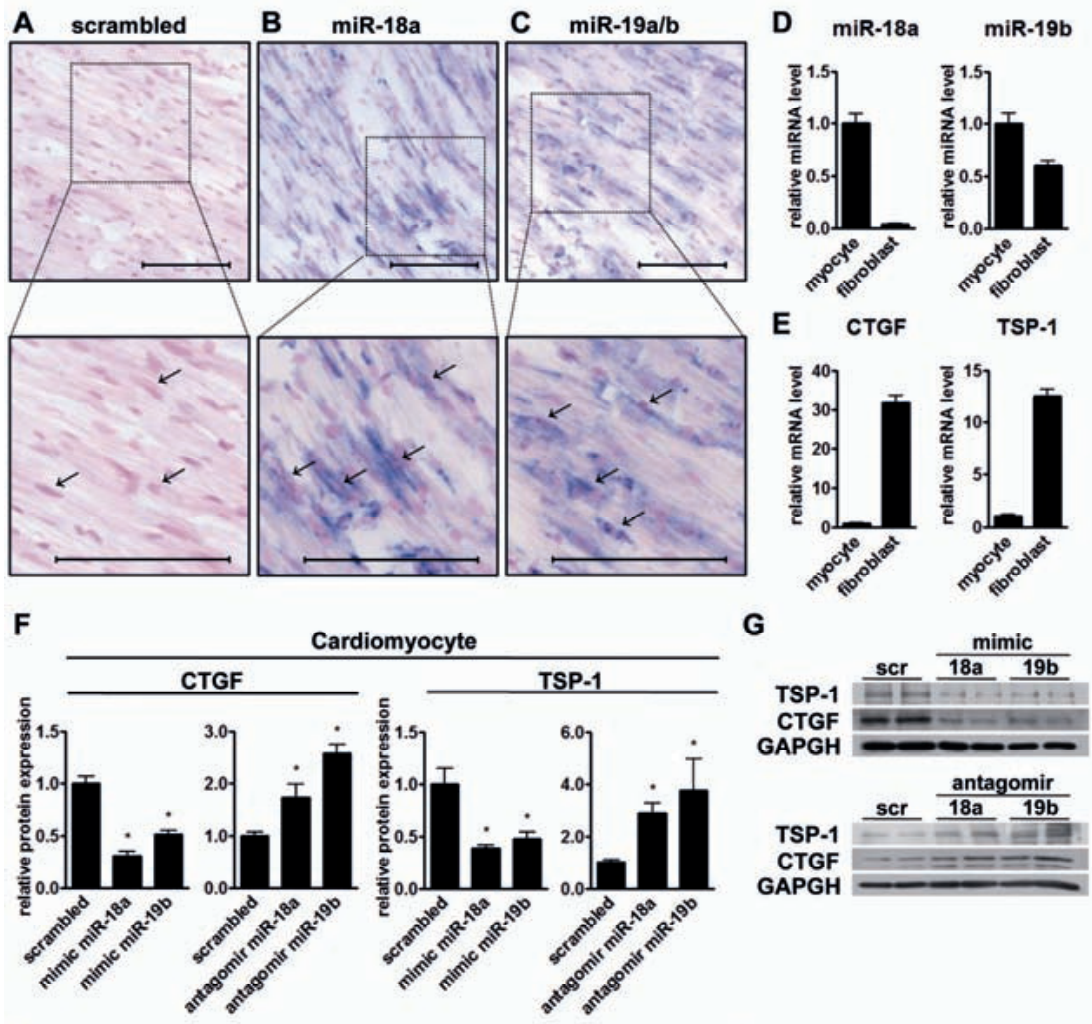

G

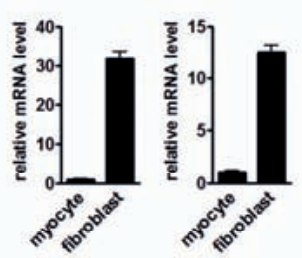

H

Fibroblast
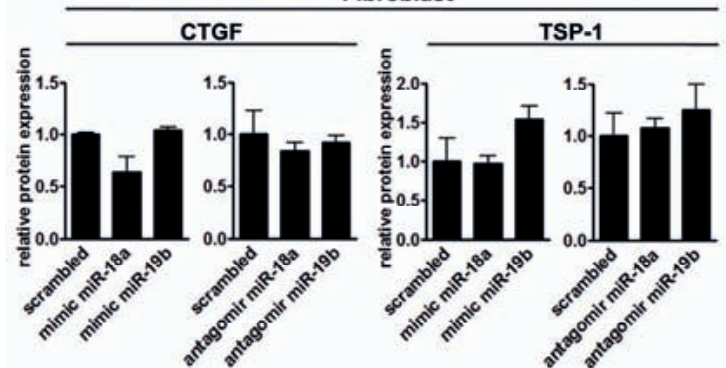

I

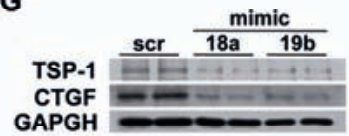

GAPGH

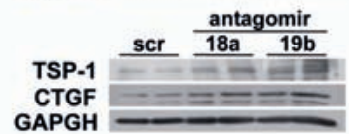

GAPGH

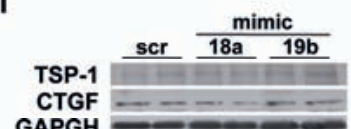

GAPGH

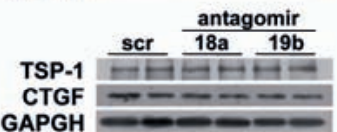

Figure 5. MiR-18a and miR-19b regulate CTGF and TSP-1 expression in cardiomyocytes. (A-C) In situ hybridization showed the abundant expression of miR-18a and miR-19b in the myocardium of adult C57BI6 mice, most of it expressed by cardiomyocytes. Black arrows indicate the cardiomyocyte nucleus and illustrate the perinuclear localization of these miRNAs. ( $D$ and $E$ ) Comparison of the expression profiles in cultured NRCMs and NRCFs shows that abundant expression of miR-18a and miR-19b in NRCMs is paralleled by low CTGF and TSP-1 transcript levels. (F and G) Immunoblotting revealed that manipulating miR$18 \mathrm{a}$ and miR-19b function by overexpression of these miRNAs using mimics in NRCMs was sufficient to decrease CTGF and TSP-1 protein expression, while inhibition with antagomirs enhanced CTGF and TSP-1 levels. ( $\mathrm{H}$ and $\mathrm{I}$ ) In contrast to NRCMs, immunoblotting in cultured NRCFs showed that CTGF and TSP-1 protein expression was not suppressed by overexpression of miR-18a and miR-19b, nor did inhibition of these miRNAs result in increased CTGF and TSP-1 levels. Mimic and antagomir experiments were performed with $n=4$ per group and data were normalized for GAPDH expression. Data were presented as mean \pm SEM. ${ }^{*} P \leq 0.05$ versus scrambled control oligonucleotides. 
CTGF and TSP-1 are pro-fibrotic ${ }^{29,30}$, and therefore we investigated whether their regulating miRs-18a and miR-19b were also capable of affecting collagen production. Indeed, overexpression of miR18a and miR-19b in cardiomyocytes repressed collagen $1 A 1$ and 3A1 mRNA levels, while inhibition of these miRNAs using antagomirs significantly enhanced collagen transcription (Figure 6A). In contrast, collagen $1 A 1$ and 3A1 transcription was not affected by either miR-18a and miR-19b overexpression or inhibition in NRCFs, indicating that collagen expression in cardiac fibroblasts is unrelated to these miRNAs (Figure 6B). Thus, in concordance to CTGF and TSP-1 regulation by miR-18a and miR-19b in cardiomyocytes, these data strongly imply that miR-18a and miR-19b contribute to the induction of collagen synthesis in aged cardiomyocytes via the regulation of the pro-fibrotic CTGF and TSP-1.

\section{A}

\section{Cardiomyocyte}
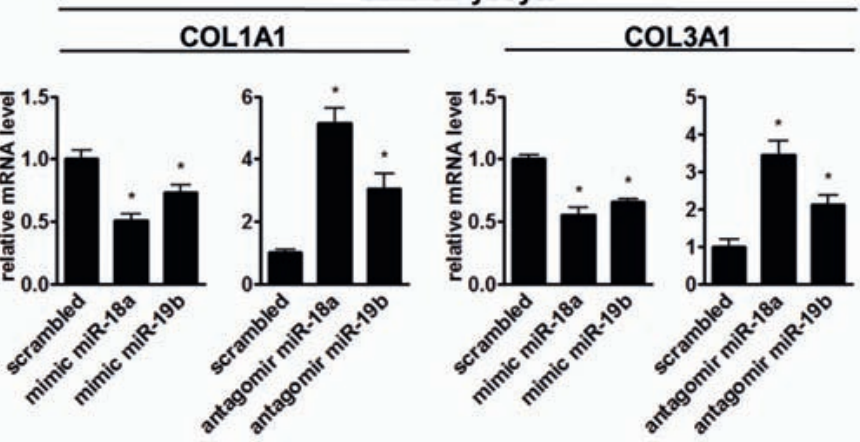

B

Fibroblast
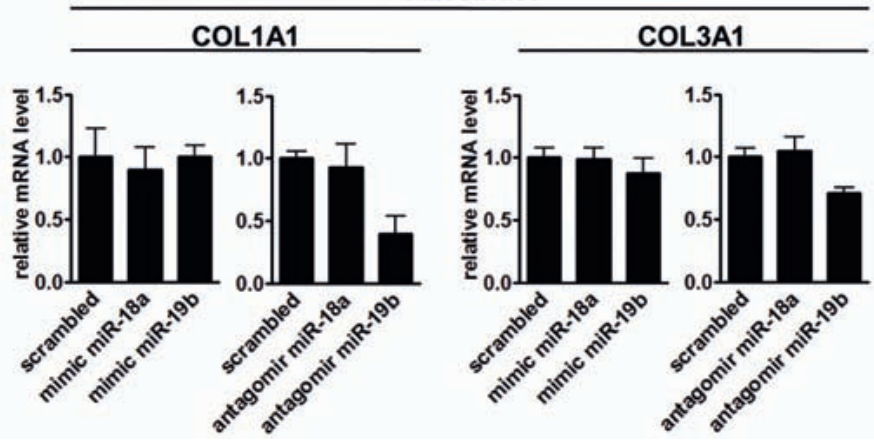

Figure 6. MiR-18a and miR-19b regulate collagen $1 \mathrm{~A} 1$ and 3A1 expression in cardiomyocytes in vitro. RTPCR analysis for the induction collagen 1A1 (COL1A1) and collagen 3A1 (COL3A1) in cultured NRCMs and NRCFs after manipulation with miR-18a and miR-19b mimics and antagomirs. (A) Overexpression of miR$18 \mathrm{a}$ and miR-19b in cardiomyocytes significantly reduces collagen $1 \mathrm{~A} 1$ and collagen $3 \mathrm{~A} 1$ transcript levels, while inhibition of these miRNAs using antagomirs significantly induced transcription of both collagen types. (B) Collagen $1 \mathrm{~A} 1$ and $3 \mathrm{~A} 1$ expression in cultured cardiac fibroblasts seemed unrelated to miR-18a and miR-19b, as no significant repression or induction was observed in NRCFs after treatment with miR-18a and miR-19b mimics or antagomirs respectively. All experiments were performed with $n=4$ per group and data were normalized for GAPDH transcript levels. Data were presented as mean \pm SEM. ${ }^{*} P \leq 0.05$ versus scrambled control oligonucleotides. 


\section{Discussion}

With aging, cardiac function declines as the result of cardiomyocyte loss, left ventricular hypertrophy, dilation and accumulation and remodeling of the extracellular matrix. ${ }^{1}$ It is well accepted that genetic changes significantly contribute to the aging process in the heart. $^{34,35}$ Due to the highly accessible mRNA microarray technique, most studies primarily focused on the regulation of gene expression by transcriptional control. With the discovery of miRNAs, now the emphasis is being expanded to the posttranscriptional level of regulation that gives rise to these age-specific gene profiles.

A study showing that the miRNA lin-4 and its target protein lin-14 determine lifespan in C. elegans, has paved the way for exploring the role of miRNAs in aging. ${ }^{10}$ Now, increasing evidence on miRNAs as mediators of age-associated pathologies, together with reports of age-related changes in miRNA expression in aged organs and senescent cells, point towards miRNA-regulation as a common biological mechanism that underlies aging and cellular senescence in different organs and cell types. ${ }^{11-13}$ Nevertheless, so far no studies have addressed the role of miRNAs in the old heart and aging of cardiomyocytes.

In the present study, we showed extensive changes in the expression of the miR17 92 cluster in a model of age-related heart failure in mice. We were able to extrapolate our findings to patients, which confirms the well-recognized highly conserved nature of microRNAs and their target sites. Initially the miR-17 92 cluster was linked to cancer pathogenesis, and was thought to be pro-tumorgenic because of its regulation by c-Myc. ${ }^{19}$ However, the cluster was later found to be tumor-suppressive in multiple forms of cancer. ${ }^{36}$ Tumor suppressor mechanisms can induce cellular senescence and contribute to the aging process ${ }^{37}$, turning the miR-17 92 cluster into a potential mediator of aging. Indeed, overexpression of miR-17 and miR-20a inhibited senescence in primary human fibroblasts by blunting the activation of $\mathrm{p} 21^{\text {WAF1 }}$, while inhibition of miR-17 caused senescence in anaplastic thyroid cancer cells. ${ }^{38,}{ }^{39}$ Furthermore, miR17 92 expression is consistently down-regulated in multiple models of aging, i.e. after irradiation $^{40}$, p53 induction ${ }^{41}$ or stress-induced senescence ${ }^{42}$, and in old human skin, bone-marrow derived mesenchymal stem cells, T-cells ${ }^{43}$ and peripheral blood mononuclear cells. ${ }^{44}$ These reports are in line with our data showing repression of the miR17 92 cluster in old failing hearts. In addition, we demonstrate that the miR-17 92 cluster is part of the senescence-signature of the aged cardiomyocyte.

From the six members of the miR-17 92 cluster, miR-18a, miR-19a and miR-19b were among the most strongly repressed miRNAs in aged cardiomyocytes and hearts of old failure prone mice. Several studies have linked these specific cluster members, and not the other cluster members, to the matricellular proteins CTGF and TSP-1. ${ }^{19,21,}$ ${ }^{31}$. Interestingly, CTGF upregulation in age-induced cardiac disease correlates with TGF$\beta$ induction, cardiac fibrosis and left ventricular stiffening. ${ }^{8,45}$ The role of TSP-1 in cardiac aging was not reported so far, but another member of the family of thrombospondins, TSP-2, was recently shown to be up-regulated in aged hearts and to pro- 
tect against age-related cardiac dysfunction. ${ }^{7}$ Here, we showed increased expression of CTGF and TSP-1 in age-related HF both in mice and human.

Traditionally it has been assumed that CTGF and TSP-1 are predominantly expressed by fibroblasts in the heart. However, several studies have recognized that during cardiac remodeling CTGF and TSP-1 are also secreted by cardiomyocytes to regulate their surrounding ECM environment. ${ }^{46-48}$ Moreover, a role for miRNAs in regulating CTGF expression in cardiomyocytes has been established by Duisters et al., who showed that increased CTGF transcription during pathological LV hypertrophy in (young) hearts is controlled by miR-30 and miR-133. ${ }^{49}$ Our in vitro results support a role for miR-18a, miR-19a and miR-19b in regulating CTGF and TSP-1 expression in the aged cardiomyocyte. Here, miRNA mimics of miR-18a and miR-19b blunted the expression of CTGF and TSP-1, and vice versa inhibition of these miRNAs enhanced CTGF and TSP-1 levels. In cardiac fibroblasts, overexpression of miR-18a and miR-19b also decreased CTGF and TSP-1 transcription, however, inhibition of these miRNAs was not sufficient to increase CTGF and TSP-1. This may be due to the fact that a fibroblast produces large amounts of CTGF and TSP-1 while it contains relatively low amounts of miR-18a and miR-19b. These results imply that the age-related regulation of CTGF and TSP-1 expression by miR-18a and miR-19b in the heart is uniquely restricted to the cardiomyocyte in order to control its surrounding ECM.

In conclusion, our study is the first to show that miRNA expression of the miR17 92 cluster changes with cardiac aging, and associates decreased miR-18a, miR-19a, and miR-19b expression with age-related remodeling in the heart. Our results suggest that upregulation of these miRNAs in the aged failure-protected heart blunts the expression of TSP-1 and CTGF in order to dampen the fibrotic remodeling process that contributes to the functional decline with age. Although we do not show direct regulation of CTGF and TSP-1 by these miRNAs, previous data have proven a direct mechanism. ${ }^{21,31}$ This study provides evidence for the involvement of miRNAs in regulating cardiac aging and identifies them as potential new therapeutic targets for the modulation of aging-induced cardiac remodeling.

\section{Acknowledgments}

The authors thank Hans Duimel for his excellent technical support in electron microscopy and Wim Engels for his assistance in designing a method to measure lipofuscin accumulation in cardiomyocytes in vitro using a combination of 2-photon and confocal microscopy. Robert Dennert collected and provided the human cardiac biopsy specimens. Marc F. Hoylaerts (Katholieke Universiteit Leuven, Belgium) kindly provided the rabbit anti-human TSP-1 antibody. 


\section{References}

1 Lakatta EG, Sollott SJ. Perspectives on mammalian cardiovascular aging: humans to molecules. Comp Biochem Physiol A Mol Integr Physiol. 2002;132(4):699-721.

2 Barasch E, Gottdiener JS, Aurigemma G, Kitzman DW, Han J, Kop WJ, Tracy RP. Association between elevated fibrosis markers and heart failure in the elderly: the cardiovascular health study. Circ Heart Fail. 2009;2(4):303-310.

3 Boyle AJ, Shih H, Hwang J, Ye J, Lee B, Zhang Y, Kwon D, Jun K, Zheng D, Sievers R, Angeli F, Yeghiazarians $Y$, Lee R. Cardiomyopathy of Aging in the Mammalian Heart is Characterized by Myocardial Hypertrophy, Fibrosis and a Predisposition Towards Cardiomyocyte Apoptosis and Autophagy. Exp Gerontol. 2011.

4 Capasso JM, Palackal T, Olivetti G, Anversa P. Severe myocardial dysfunction induced by ventricular remodeling in aging rat hearts. Am J Physiol. 1990;259(4 Pt 2):H1086-1096.

5 Bradshaw AD, Baicu CF, Rentz TJ, Van Laer AO, Bonnema DD, Zile MR. Age-dependent alterations in fibrillar collagen content and myocardial diastolic function: role of SPARC in post-synthetic procollagen processing. Am J Physiol Heart Circ Physiol. 2010;298(2):H614-622.

6 Burgess ML, McCrea JC, Hedrick HL. Age-associated changes in cardiac matrix and integrins. Mech Ageing Dev. 2001;122(15):1739-1756.

7 Swinnen M, Vanhoutte D, Van Almen GC, Hamdani N, Schellings MW, D'Hooge J, Van der Velden J, Weaver MS, Sage EH, Bornstein P, Verheyen FK, VandenDriessche T, Chuah MK, Westermann D, Paulus WJ, Van de Werf F, Schroen B, Carmeliet P, Pinto YM, Heymans S. Absence of thrombospondin-2 causes age-related dilated cardiomyopathy. Circulation. 2009;120(16):1585-1597.

8 Wang M, Zhang J, Walker SJ, Dworakowski R, Lakatta EG, Shah AM. Involvement of NADPH oxidase in age-associated cardiac remodeling. J Mol Cell Cardiol. 2010;48(4):765-772.

9 Panek AN, Posch MG, Alenina N, Ghadge SK, Erdmann B, Popova E, Perrot A, Geier C, Dietz R, Morano I, Bader M, Ozcelik C. Connective tissue growth factor overexpression in cardiomyocytes promotes cardiac hypertrophy and protection against pressure overload. PLoS One. 2009;4(8):e6743.

10 Boehm M, Slack F. A developmental timing microRNA and its target regulate life span in C. elegans. Science. 2005;310(5756):1954-1957.

11 Grillari J, Grillari-Voglauer R. Novel modulators of senescence, aging, and longevity: Small non-coding RNAs enter the stage. Exp Gerontol. 2010;45(4):302-311.

12 Chen LH, Chiou GY, Chen YW, Li HY, Chiou SH. microRNA and aging: a novel modulator in regulating the aging network. Ageing Res Rev. 2010;9 Suppl 1:S59-66.

13 Mudhasani R, Zhu Z, Hutvagner G, Eischen CM, Lyle S, Hall LL, Lawrence JB, Imbalzano AN, Jones SN. Loss of miRNA biogenesis induces p19Arf-p53 signaling and senescence in primary cells. J Cell Biol. 2008;181(7):1055-1063.

14 van Rooij E, Sutherland LB, Liu N, Williams AH, McAnally J, Gerard RD, Richardson JA, Olson EN. A signature pattern of stress-responsive microRNAs that can evoke cardiac hypertrophy and heart failure. Proc Natl Acad Sci U S A. 2006;103(48):18255-18260.

15 Thum T, Galuppo P, Wolf C, Fiedler J, Kneitz S, van Laake LW, Doevendans PA, Mummery CL, Borlak J, Haverich A, Gross C, Engelhardt S, Ertl G, Bauersachs J. MicroRNAs in the human heart: a clue to fetal gene reprogramming in heart failure. Circulation. 2007;116(3):258-267.

16 van Rooij E, Sutherland LB, Qi X, Richardson JA, Hill J, Olson EN. Control of stress-dependent cardiac growth and gene expression by a microRNA. Science. 2007;316(5824):575-579.

17 Zhao Y, Ransom JF, Li A, Vedantham V, von Drehle M, Muth AN, Tsuchihashi T, McManus MT, Schwartz RJ, Srivastava D. Dysregulation of cardiogenesis, cardiac conduction, and cell cycle in mice lacking miRNA-1-2. Cell. 2007;129(2):303-317.

18 da Costa Martins PA, Salic K, Gladka MM, Armand AS, Leptidis S, el Azzouzi H, Hansen A, Coenen-de Roo CJ, Bierhuizen MF, van der Nagel R, van Kuik J, de Weger R, de Bruin A, Condorelli G, Arbones ML, Eschenhagen T, De Windt LJ. MicroRNA-199b targets the nuclear kinase Dyrk1a in an auto-amplification loop promoting calcineurin/NFAT signalling. Nat Cell Biol. 2010;12(12):1220-1227. 
19 Dews M, Homayouni A, Yu D, Murphy D, Sevignani C, Wentzel E, Furth EE, Lee WM, Enders GH, Mendell JT, Thomas-Tikhonenko A. Augmentation of tumor angiogenesis by a Myc-activated microRNA cluster. Nat Genet. 2006;38(9):1060-1065.

20 Shan SW, Lee DY, Deng Z, Shatseva T, Jeyapalan Z, Du WW, Zhang Y, Xuan JW, Yee SP, Siragam V, Yang BB. MicroRNA MiR-17 retards tissue growth and represses fibronectin expression. Nat Cell Biol. 2009;11(8):1031-1038.

21 Suarez Y, Fernandez-Hernando C, Yu J, Gerber SA, Harrison KD, Pober JS, Iruela-Arispe ML, Merkenschlager M, Sessa WC. Dicer-dependent endothelial microRNAs are necessary for postnatal angiogenesis. Proc Natl Acad Sci U S A. 2008;105(37):14082-14087.

22 He L, Thomson JM, Hemann MT, Hernando-Monge E, Mu D, Goodson S, Powers S, Cordon-Cardo C, Lowe SW, Hannon GJ, Hammond SM. A microRNA polycistron as a potential human oncogene. Nature. 2005;435(7043):828-833.

23 van Haaften G, Agami R. Tumorigenicity of the miR-17-92 cluster distilled. Genes Dev. 2010;24(1):1-4.

24 Ventura A, Young AG, Winslow MM, Lintault L, Meissner A, Erkeland SJ, Newman J, Bronson RT, Crowley $D$, Stone JR, Jaenisch R, Sharp PA, Jacks T. Targeted deletion reveals essential and overlapping functions of the miR-17 through 92 family of miRNA clusters. Cell. 2008;132(5):875-886.

25 De Windt LJ, Willemsen PH, Popping S, Van der Vusse GJ, Reneman RS, Van Bilsen M. Cloning and cellular distribution of a group II phospholipase A2 expressed in the heart. J Mol Cell Cardiol. 1997;29(8):2095-2106.

26 Moura R, Tjwa M, Vandervoort P, Van Kerckhoven S, Holvoet P, Hoylaerts MF. Thrombospondin-1 deficiency accelerates atherosclerotic plaque maturation in ApoE-/- mice. Circ Res. 2008;103(10):11811189.

27 Nuovo GJ. In situ detection of microRNAs in paraffin embedded, formalin fixed tissues and the colocalization of their putative targets. Methods. 2010;52(4):307-315.

28 Kemi M, Keenan KP, McCoy C, Hoe CM, Soper KA, Ballam GC, van Zwieten MJ. The relative protective effects of moderate dietary restriction versus dietary modification on spontaneous cardiomyopathy in male Sprague-Dawley rats. Toxicol Pathol. 2000;28(2):285-296.

29 Shi-Wen X, Leask A, Abraham D. Regulation and function of connective tissue growth factor/CCN2 in tissue repair, scarring and fibrosis. Cytokine Growth Factor Rev. 2008;19(2):133-144.

30 Belmadani S, Bernal J, Wei CC, Pallero MA, Dell'italia L, Murphy-Ullrich JE, Berecek KH. A thrombospondin-1 antagonist of transforming growth factor-beta activation blocks cardiomyopathy in rats with diabetes and elevated angiotensin II. Am J Pathol. 2007;171(3):777-789.

31 Ohgawara T, Kubota S, Kawaki H, Kondo S, Eguchi T, Kurio N, Aoyama E, Sasaki A, Takigawa M. Regulation of chondrocytic phenotype by micro RNA 18a: involvement of Ccn2/Ctgf as a major target gene. FEBS Lett. 2009;583(6):1006-1010.

32 Paulus WJ, Tschope C, Sanderson JE, Rusconi C, Flachskampf FA, Rademakers FE, Marino P, Smiseth OA, De Keulenaer G, Leite-Moreira AF, Borbely A, Edes I, Handoko ML, Heymans S, Pezzali N, Pieske B, Dickstein K, Fraser AG, Brutsaert DL. How to diagnose diastolic heart failure: a consensus statement on the diagnosis of heart failure with normal left ventricular ejection fraction by the Heart Failure and Echocardiography Associations of the European Society of Cardiology. Eur Heart J. 2007;28(20):2539-2550.

33 Terman A, Brunk UT. Aging as a catabolic malfunction. Int J Biochem Cell Biol. 2004;36(12):2365-2375.

34 Bodyak N, Kang PM, Hiromura M, Sulijoadikusumo I, Horikoshi N, Khrapko K, Usheva A. Gene expression profiling of the aging mouse cardiac myocytes. Nucleic Acids Res. 2002;30(17):3788-3794.

35 Park SK, Prolla TA. Gene expression profiling studies of aging in cardiac and skeletal muscles. Cardiovasc Res. 2005;66(2):205-212.

36 Zhang L, Huang J, Yang N, Greshock J, Megraw MS, Giannakakis A, Liang S, Naylor TL, Barchetti A, Ward MR, Yao G, Medina A, O’Brien-Jenkins A, Katsaros D, Hatzigeorgiou A, Gimotty PA, Weber BL, Coukos G. microRNAs exhibit high frequency genomic alterations in human cancer. Proc Natl Acad Sci U S A. 2006;103(24):9136-9141.

37 Campisi J. Cancer and ageing: rival demons? Nat Rev Cancer. 2003;3(5):339-349.

38 Hong L, Lai M, Chen M, Xie C, Liao R, Kang YJ, Xiao C, Hu WY, Han J, Sun P. The miR-17-92 Cluster of microRNAs Confers Tumorigenicity by Inhibiting Oncogene-Induced Senescence. Cancer Res. 2010. 
39 Takakura S, Mitsutake N, Nakashima M, Namba H, Saenko VA, Rogounovitch TI, Nakazawa Y, Hayashi T, Ohtsuru A, Yamashita S. Oncogenic role of miR-17-92 cluster in anaplastic thyroid cancer cells. Cancer Sci. 2008;99(6):1147-1154.

40 Maes OC, An J, Sarojini H, Wu H, Wang E. Changes in MicroRNA expression patterns in human fibroblasts after low-LET radiation. J Cell Biochem. 2008;105(3):824-834.

41 Brosh R, Shalgi R, Liran A, Landan G, Korotayev K, Nguyen GH, Enerly E, Johnsen H, Buganim Y, Solomon H, Goldstein I, Madar S, Goldfinger N, Borresen-Dale AL, Ginsberg D, Harris CC, Pilpel Y, Oren M, Rotter V. p53-Repressed miRNAs are involved with E2F in a feed-forward loop promoting proliferation. Mol Syst Biol. 2008;4:229.

42 Li G, Luna C, Qiu J, Epstein DL, Gonzalez P. Alterations in microRNA expression in stress-induced cellular senescence. Mech Ageing Dev. 2009;130(11-12):731-741.

43 Hackl M, Brunner S, Fortschegger K, Schreiner C, Micutkova L, Muck C, Laschober GT, Lepperdinger G, Sampson N, Berger P, Herndler-Brandstetter D, Wieser M, Kuhnel H, Strasser A, Rinnerthaler M, Breitenbach M, Mildner M, Eckhart L, Tschachler E, Trost A, Bauer JW, Papak C, Trajanoski Z, Scheideler M, Grillari-Voglauer R, Grubeck-Loebenstein B, Jansen-Durr P, Grillari J. miR-17, miR-19b, miR-20a, and miR106a are down-regulated in human aging. Aging Cell. 2010;9(2):291-296.

44 Noren Hooten N, Abdelmohsen K, Gorospe M, Ejiogu N, Zonderman AB, Evans MK. microRNA expression patterns reveal differential expression of target genes with age. PLoS One.5(5):e10724.

45 Koitabashi N, Arai M, Kogure S, Niwano K, Watanabe A, Aoki Y, Maeno T, Nishida T, Kubota S, Takigawa $\mathrm{M}$, Kurabayashi $\mathrm{M}$. Increased connective tissue growth factor relative to brain natriuretic peptide as a determinant of myocardial fibrosis. Hypertension. 2007;49(5):1120-1127.

46 Chen MM, Lam A, Abraham JA, Schreiner GF, Joly AH. CTGF expression is induced by TGF- beta in cardiac fibroblasts and cardiac myocytes: a potential role in heart fibrosis. J Mol Cell Cardiol. 2000;32(10):18051819.

47 Frangogiannis NG, Ren G, Dewald O, Zymek P, Haudek S, Koerting A, Winkelmann K, Michael LH, Lawler J, Entman ML. Critical role of endogenous thrombospondin-1 in preventing expansion of healing myocardial infarcts. Circulation. 2005;111(22):2935-2942.

48 Ohnishi H, Oka T, Kusachi S, Nakanishi T, Takeda K, Nakahama M, Doi M, Murakami T, Ninomiya Y, Takigawa M, Tsuji T. Increased expression of connective tissue growth factor in the infarct zone of experimentally induced myocardial infarction in rats. J Mol Cell Cardiol. 1998;30(11):2411-2422.

49 Duisters RF, Tijsen AJ, Schroen B, Leenders JJ, Lentink V, van der Made I, Herias V, van Leeuwen RE, Schellings MW, Barenbrug P, Maessen JG, Heymans S, Pinto YM, Creemers EE. miR-133 and miR-30 regulate connective tissue growth factor: implications for a role of microRNAs in myocardial matrix remodeling. Circ Res. 2009;104(2):170-178, 176p following 178. 


\title{
Chapter 5
}

\section{Supplemental material}

\author{
Expanded methods
}

Mice

Male C57BI6 mice were obtained from Janvier (Le Genest Saint Isle, France) and mice on a mixed genetic background (C57BI6 $\times 129 \mathrm{~Sv}$ ) were backcrossed four times into the C57BI6 background within the animal facility of the University of Maastricht. All animals were housed under standard day-night rhythm and ad libitum conditions until 12, 52 and 104 weeks of age. Cardiac function was assessed at the end of the experiment under sedation ( $2 \%$ isoflurane) using a $12-\mathrm{MHz}$ transducer (Hewlett Packard) on a Sonos 5500 (Hewlett Packard) echocardiograph. Echocardiographic images were taken in B-mode to analyse fractional shortening (FS), left ventricular diameters at enddiastole (LVEDd) and end-systole (LVEDs), left ventricular posterior wall at end-diastole (LVPWd) and end-systole (LVPWs), and intraventricular septal thickness at end-diastole (IVSd) and end-systole (IVSs). Subsequent to echocardiography, hearts were taken out and prepared for molecular, histological and ultrastructural analysis.

The present study was approved by the Institutional Animal Research Committee and conforms with the guidelines for the use of laboratory animals formulated in the Dutch law on care and use of experimental animals.

\section{Histology and electron microscopy}

After removal, hearts were cut transversely, fixed in $1 \%$ buffered paraformaldehyde and embedded in paraffin for histological examination. To measure collagen in the hearts of 12,52 , and 104 weeks old mice, four $\mu \mathrm{m}$ thick paraffin sections were stained with Sirius red. Interstitial collagen content was determined as percentage Sirius red staining per total cardiac tissue area, and perivascular fibrosis was presented as percentage collagen in the area adjacent to the vasculature. All morphometric analyses were performed using a Leica DMRXE microscope (Leica Microsystems Cambridge Ltd) in combination with Leica Qwin image processing and analysis software (Leica. Microsystems Cambridge Ltd).

For high magnification images, hearts were fixed in $2.5 \%$ glutaraldehyde in $0.1 \mathrm{M}$ phosphate buffer at $\mathrm{pH} 7.4$, and post-fixed in $1 \%$ osmiumtetroxide in $0.067 \mathrm{M}$ cacodylate buffer. After fixation, the samples were routinely dehydrated in graded ethanol series (70\%, 90\% and 100\%), followed by embedding in Epon. Electron microscopic 
images were made from $80 \mathrm{~nm}$ thick sections with a Philips CM100 (F.E.I., Eindhoven, The Netherlands) at an accelerating voltage of $80 \mathrm{KV}$.

\section{Patients}

Nineteen subjects diagnosed with idiopathic cardiomyopathy (ICM) were included based on age and cardiac function. Five old patients (60.5 years \pm 0.8 ) with preserved cardiac function (ejection fraction between 40 and 55 percent) and no signs of cornary artery disease (ejection fraction (EF): $45.6 \% \pm 9.2$ ), and nine ICM patients at older age (67.0 years \pm 4.3 ) with a severely compromised cardiac function (EF: $18.9 \% \pm 3.0$ ) were compared to a group of five young patients (33.2 years \pm 4.4 ) with preserved cardiac function (EF: $46.5 \% \pm 4.4$ ). All patients had no unstable angina, and no history of myocardial infarction or left ventricular hypertrophy. Three transmural needle biopsies were taken from the left ventricular anterior wall, and snap-frozen in liquid nitrogen for further molecular analysis. This study occurred in line with the recommendations of the institutional ethics committee of the University Hospital Maastricht.

\section{Neonatal cardiac cell isolation}

Neonatal rat cardiomyocytes (NRCMs) and cardiac fibroblasts (NRCFs) were isolated from 1- to 3-day-old Lewis rats as described previously. ${ }^{1}$ Briefly, cardiac cells were isolated by collagenase digestion from the ventricles. After 60 minutes of preplating, the non-attached NRCMs were separated from the NRCFs and plated in gelatinized sixwell plates and cultured overnight in Dulbecco's modified Eagle's medium (DMEM) (Invitrogen, Carlsbad, CA, USA) supplemented with 10\% horse serum (PAA, Pasching, Austria), 5\% heat-inactivated newborn calf serum (PAA, Pasching, Austria), M-199 (Invitrogen, Carlsbad, CA, USA), glucose (Merck, Darmstadt, Germany), gentamycin (Invitrogen, Carlsbad, CA, USA), and Penicillin Streptomycin (Invitrogen, Carlsbad, CA, USA). NRCMs were cultured in the presence of cytosine $\beta$-D-arabinofuranoside (Sigma, St Louis, MO, USA) to suppress proliferation of non-cardiomyocytes. NRCFs were cultured in DMEM (Invitrogen, Carlsbad, CA, USA), supplemented with 10\% fetal bovine serum (PAA, Pasching, Austria) and gentamycin (Invitrogen, Carlsbad, CA, USA).

\section{Lipofuscin accumulation in aged cardiomyocytes in vitro.}

After isolation, $7.5 \times 10^{5} \mathrm{NRCMs}$ per well were plated in six well plates and cultured for 4 and 21 days. Cardiomyocytes were cultured under high serum conditions ( $10 \%$ horse serum and $5 \%$ heat-inactivated newborn calf serum) as described in the section above. The medium was changed 24 hours after isolation and then every two days.

After 4 and 21 days, lipofuscin accumulation was determined using a combination of confocal and two-photon microscopy. Briefly, the medium was removed and NRCMs were incubated for 5 minutes with prewarmed, 37 degrees phosphate buffered saline 
(PBS) supplemented with $1 \mu \mathrm{M}$ Hoechst 33342 (Fluka, Sigma-Aldrich Corp., St. Louis, MO, USA). Images were captured with an Eclipse E600FN upright microscope (Nikon, Tokyo, Japan) equipped with a Radiance 2100MP optical imaging system (Bio-Rad, Hercules, CA, USA). Hoechst-stained nuclei were excited with a two-photon SpectraPhysics Tsunami laser (Spectra-Physics, Irvine, CA, USA) centered at $800 \mathrm{~nm}$ and visualized at 400-480 $\mathrm{nm}$. Subsequently, autofluorescent lipofuscin was excited using a laser at $488 \mathrm{~nm}$, with confocal detection at 510-560 nm. Images were created with LaserSharp 5.0 software (Bio-Rad, Hercules, CA, USA) and lipofuscin accumulation per cardiomyocyte was determined using Leica Qwin image processing and analysis software (Leica. Microsystems Cambridge Ltd, Cambridge, UK). After measuring the lipofuscin content cells were pelleted and snap frozen in liquid nitrogen for RNA extraction.

\section{Transfection of cultured cardiomyocytes and cardiac fibroblasts}

For miRNA mimic (overexpression) or antagomir (inhibition) transfection, $7.5 \times 10^{5}$ NRCMs and $1.5 \times 10^{5}$ NRCFs per well were plated in a six-well plate and incubated overnight. The next day cells were placed on DMEM under low serum conditions for 24 hours before the medium was changed to serum-free DMEM without antibiotics for 4 hours. NRCMs and NRCFs were then transfected with $80 \mathrm{~nm}$ miRIDIAN hairpin inhibitor miR-18a (\#IH-300487-06), miRIDIAN mimic miR-18a (\#C-300487-05), miRIDIAN hairpin inhibitor miR-19b (\#IH-300489-05), miRIDIAN mimic miR-19b (\#C-300489-03), miRIDIAN microRNA hairpin inhibitor negative control \#1 (\#IN-001005-01), and miRIDIAN microRNA mimic negative control \#2 (\#CN-002000-01) (Dharmacon, Colorado, CO, USA), fascilitated by lipofectamine 2000 (Invitrogen, Carlsbad, CA, USA) according to the manufacturer's protocol. After 48 hours, cells were trypsinized, pelleted, and snap frozen in liquid nitrogen for RNA isolation.

\section{RNA isolation and Real-Time PCR}

Total RNA was extracted from homogenized cells and heart tissues using the mirVANA miRNA isolation kit (Ambion, Austin, TX, USA) according to the manufacturer's protocol followed by removal of genomic DNA with the DNA-free ambion kit (Ambion, Austin, TX, USA). cDNA was generated from 500 nanograms of total RNAwith the qScript cDNA synthesis kit (Quanta BioSciences Inc., Gaithersburg, MD, USA) and miScript reverse transcription kit (Qiagen, Hilden, Germany) for the detection of gene transcript levels or miRNA expression respectively. Five nanograms of cDNA template was amplified with the MylQ Single Color Real-Time PCR detection System (Bio-Rad, Hercules, CA, USA) to measure transcript levels of CTGF, TSP-1, collagen $1 A 1$ and collagen $3 A 1$ (primer sequences are listed in supplemental table 3 ), and miRNA expression of miR-17, miR18a, miR-19a, miR-19b-1, miR-20, and miR-92a-1 using the specific miScript Primer Assays (Qiagen, Hilden, Germany). Expression levels were analyzed with BioRad iQ5 
software (Bio-Rad, Hercules, CA, USA) and presented relative to glyceraldehydes-3phosphate dehydrogenase (GAPDH).

\section{Immunoblotting}

Left ventricular heart tissue was homogenized (RyboLyser, Hybaid, UK) and extracted in homogenization buffer $\left(50 \mathrm{mM}\right.$ Tris- $\mathrm{HCl} \mathrm{pH} 7.5,10 \mathrm{mM} \mathrm{CaCl}$, and $10 \mu \mathrm{M} \mathrm{ZnCl}_{2}$ ), diluted in 2x sample buffer (0.5 M Tris- $\mathrm{HCl}$ pH6.8, 20\% glycerol, 4\% SDS, $1 \mathrm{mg}$ bromophenol blue, $\mathrm{H}_{2} \mathrm{O}$, and 2-mercaptoethanol) and boiled for 5 minutes at $95^{\circ} \mathrm{C}$. NRCMs and NRCFs were harvested in a identical manner. The protein lysates (10 $\mu \mathrm{g}$ of protein) were separated by SDS-PAGE (10\% running, $4 \%$ stacking) and transferred to a polyvinylidene fluoride membrane (Immobilon-P, $0.45 \mu \mathrm{m}$ pore size). After blocking in 3.5\% protifar plus (Nutricia), the membranes were probed overnight at $4^{\circ} \mathrm{C}$ with a primary antibody to detect CTGF (dilution 1:500) (GeneTex Inc., Irvine, CA, USA; \#GTX26992), TSP-1 $(5 \mu \mathrm{g} / \mathrm{ml}$ ) (in-house rabbit anti-human TSP-1 was kindly provided by MF Hoylaerts ${ }^{2}$, University of Leuven, Belgium), and glyceraldehyde-3-phosphate dehydrogenase (GAPDH) (dilution 1:10000) (Fitzgerald Inc., Concord, MA, USA; \#RDI-TRK5G4$6 C 5)$. Pending on the origin of the first antibody, membranes were incubated with a horseradish-linked polyclonal goat anti-rabbit (dilution 1:2000) (Cell Signaling Technology, Danvers, MA, USA; \#7074) or rabbit anti-mouse antibody (dilution 1:2000) (DAKO, Glostrup, Denmark; \#P0161) followed by visualization of the signal using Enhanced Chemi Luminiscence. Protein levels were determined using Quantity One software (Bio-Rad Laboratories Inc.) and presented relative to GAPDH protein expression.

\section{In Situ Hybridization}

Mouse left ventricular heart tissue was fixed in 4 percent paraformaldehyde for eight hours and embedded in tissuetek. Next, twelve $\mu \mathrm{m}$ thick cryosections were cut, dried to the air, and pretreated with pepsin $(1.3 \mathrm{mg} / \mathrm{ml})$ for twenty minutes prior to miRNA in situ hybridization as described previously. ${ }^{3}$ Double DIG-labeled Locked Nucleic Acid (LNA) hybridization probes complementary to mouse mature miR-18a (5DIGN/CTATCTGCACTAGATGCACCTTA/3DIG_N) (\#38462-15), miR-19b (5DIGN/TCAGTTTTGCATGGATTTGCACA/3DIG_N) (\#38092-15), and a scrambled probe (5DIGN/GTGTAACACGTCTATACGCCCA/3DIG_N) (\#99004-15) were purchased from Exiqon (Vedbaek, Denmark). Probes were used at a concentration of $200 \mathrm{mM} / \mu \mathrm{l}$.

\section{Statistical analysis}

All data are expressed as mean \pm standard error of the mean (SEM). Differences between groups were evaluated by Student's $t$-test or one-way ANOVA with Bonferroni post-hoc test when appropriate. Differences in interstitial and perivascular fibrosis 
were analyzed by two-way ANOVA and Bonferroni post-hoc test. Probability values $<0.05$ were considered statistically significant. 


\section{Supplemental tables}

Supplemental table 1. Fold change expression profiles of miR-17 92 cluster members in HF resistant and HF prone mice at different ages

\begin{tabular}{lcccccc}
\hline & \multicolumn{3}{c}{ HF resistant } & HF prone \\
\hline & $\begin{array}{c}12 \text { weeks } \\
\mathrm{n}=8\end{array}$ & $\begin{array}{c}52 \text { weeks } \\
\mathrm{n}=8\end{array}$ & $\begin{array}{c}104 \text { weeks } \\
\mathrm{n}=9\end{array}$ & $\begin{array}{c}12 \text { weeks } \\
\mathrm{n}=6\end{array}$ & $\begin{array}{c}52 \text { weeks } \\
\mathrm{n}=11\end{array}$ & $\begin{array}{c}104 \text { weeks } \\
\mathrm{n}=8\end{array}$ \\
\hline $\begin{array}{l}\text { miR-17-92 cluster } \\
\text { miR-17 }\end{array}$ & $1.00 \pm 0.13$ & $0.78 \pm 0.06$ & $0.83 \pm 0.05$ & $1.00 \pm 0.10$ & $0.81 \pm 0.05$ & $0.62 \pm 0.07^{*+\ddagger}$ \\
miR-18a & $1.00 \pm 0.14$ & $1.14 \pm 0.11$ & $1.65 \pm 0.22^{*}$ & $1.00 \pm 0.16$ & $0.80 \pm 0.07^{\ddagger}$ & $0.59 \pm 0.06^{*+\ddagger}$ \\
miR-19a & $1.00 \pm 0.16$ & $1.36 \pm 0.21$ & $1.93 \pm 0.15^{*+}$ & $1.00 \pm 0.12$ & $0.78 \pm 0.06^{\ddagger}$ & $0.72 \pm 0.06^{* \neq}$ \\
miR-19b-1 & $1.00 \pm 0.10$ & $1.28 \pm 0.15$ & $1.92 \pm 0.19^{*+}$ & $1.00 \pm 0.12$ & $0.87 \pm 0.06^{\ddagger}$ & $0.66 \pm 0.07^{* \neq}$ \\
miR-20a & $1.00 \pm 0.12$ & $0.81 \pm 0.06$ & $0.86 \pm 0.03$ & $1.00 \pm 0.11$ & $0.89 \pm 0.07$ & $0.72 \pm 0.07^{*}$ \\
miR-92a-1 & $1.00 \pm 0.09$ & $0.88 \pm 0.07$ & $1.31 \pm 0.06^{*+}$ & $1.00 \pm 0.08$ & $0.75 \pm 0.05^{*}$ & $0.68 \pm 0.05^{* \neq}$ \\
\hline
\end{tabular}

${ }^{*} P \leq 0.05$ versus 12 weeks of age with the same genotype; ${ }^{\dagger} P \leq 0.05$ versus 52 weeks of age with the same genotype; ${ }^{\ddagger} P \leq 0.05$ versus $\mathrm{HF}$ resistant mice with the same age

Supplemental table 2. Fold change expression profiles of miR-17 92 cluster members in cardiomyocytes in vitro

\begin{tabular}{lll}
\hline & \multicolumn{1}{l}{4 days } & 21 days \\
\hline miR-17-92 cluster & & \\
miR-17 & $1.00 \pm 0.19$ & $0.78 \pm 0.01$ \\
miR-18a & $1.00 \pm 0.10$ & $0.50 \pm 0.10^{*}$ \\
miR-19a & $1.00 \pm 0.11$ & $0.54 \pm 0.03^{*}$ \\
miR-19b-1 & $1.00 \pm 0.06$ & $0.47 \pm 0.03^{*}$ \\
miR-20a & $1.00 \pm 0.03$ & $0.77 \pm 0.002^{*}$ \\
miR-92a-1 & $1.00 \pm 0.08$ & $1.04 \pm 0.05$ \\
\hline
\end{tabular}

${ }^{*} P \leq 0.05$ versus 4 days old cardiomyocytes 
Supplemental table 3. Real-Time PCR primers

\begin{tabular}{|c|c|c|c|}
\hline & & Primer sequence $5^{\prime}-3^{\prime}$ & Species \\
\hline \multicolumn{4}{|l|}{ Gene } \\
\hline \multirow[t]{2}{*}{ CTGF } & $\mathrm{F}$ & cacagagtggagcgcctgttc & Human, mouse, rat \\
\hline & $\mathrm{R}$ & gatgcactttttgcccttcttaatg & \\
\hline \multirow[t]{6}{*}{ TSP-1 } & $\mathrm{F}$ & ggcaaggactgcgttggt & Human \\
\hline & $\mathrm{R}$ & cacttcacgccggcaaag & \\
\hline & $\mathrm{F}$ & gactcgggacccatctatga & Mouse \\
\hline & $\mathrm{R}$ & ggttatgattggcagctgatg & \\
\hline & $\mathrm{F}$ & gtgacggaaaatcaagtttgca & Rat \\
\hline & $\mathrm{R}$ & acttggcaccagcaaagca & \\
\hline \multirow[t]{2}{*}{ COL1A1 } & $\mathrm{F}$ & ccgcccgcacatgc & Rat \\
\hline & $\mathrm{R}$ & ctccatgttgcagtagaccttgat & \\
\hline \multirow[t]{2}{*}{ COL3A1 } & $\mathrm{F}$ & ggcaatgtaaagaagtctctgaagct & Rat \\
\hline & $\mathrm{R}$ & gtgtttgatattcaaagactgtcttgct & \\
\hline \multirow[t]{6}{*}{ GAPDH } & $\mathrm{F}$ & ccacccatggcaaattcc & Human \\
\hline & $\mathrm{R}$ & gggatttccattgatgacaag & \\
\hline & $\mathrm{F}$ & ggtggacctcatggcctaca & Mouse \\
\hline & $\mathrm{R}$ & ctctcttgctcagtgtccttgct & \\
\hline & $\mathrm{F}$ & ggtggacctcatggcctaca & Rat \\
\hline & $\mathrm{R}$ & ctctcttgctctcagtatccttgct & \\
\hline
\end{tabular}

CTGF, connective tissue growth fator; TSP-1, thrombospondin-1; COL1A1, collagen 1A1; COL3A1, collagen 3A1; GAPDH, glyceraldehyde-3-phosphate dehydrogenase. $F$ indicates forward primer and $R$ indicates reverse primer. 


\section{Supplemental figures}

A

Human CTGF
\begin{tabular}{c|c|c|c|} 
5' & \\
CDS & 3'-UTR & miR-19 \\
miR-18
\end{tabular}

Human TSP-1

5' CDS

miR-18
3'-UTR

$\mathbf{R}$

miR-19

AAAAA - 3'

B

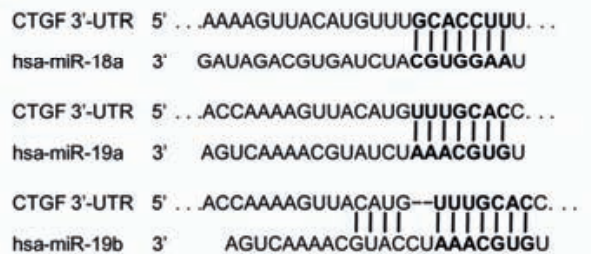

C

miR-18 target sites in CTGF 3 '-UTR

\begin{tabular}{|c|c|}
\hline Homo sapiens & 5'...UGUUUGCACCUUUCUA... 3' \\
\hline Mus musculus & 5' ... UGUUUGCACCUUUCUA... 3' \\
\hline Rattus norvegicus & $5^{\prime}$... UGUUUGCACCUUUCUA... $3^{\prime}$ \\
\hline \multicolumn{2}{|c|}{ miR-19 target sites in CTGF 3'-UTR } \\
\hline Homo sapiens & 5' ...ACAUGUUUGCACCUUU... 3' \\
\hline Mus musculus & $5^{\prime} \ldots$...CAUGUUUGCACCUUU... $3^{\prime}$ \\
\hline Rattus norvegicus & $5^{\prime}$...ACAUGUUUGCACCUUU... 3 \\
\hline \multicolumn{2}{|c|}{ miR-18 target sites in TSP-1 3'-UTR } \\
\hline Homo sapiens & $5^{\prime} \ldots$... GUAUUGCACCUUCUGG... $3^{\prime}$ \\
\hline Mus musculus & $5^{\prime} \ldots$...GCAUUGCACCUUCUAA $\ldots 3^{\prime}$ \\
\hline Rattus norvegicus & $5^{\prime}$...GCAUUGCACCUUCCGA ... 3' \\
\hline \multicolumn{2}{|c|}{ miR-19 target sites in TSP-1 3'-UTR } \\
\hline Homo sapiens & $5^{\prime} \ldots$ UAAUGUUUGCACACUGA. $3^{\prime}$ \\
\hline Mus musculus & 5 '...UG__UUGCACACUGA. 3' \\
\hline
\end{tabular}

TSP-1 3'-UTR 5' ... ACCAAUGCUGGUAUUGCACCUUC. . hsa-miR-18a 3 3. GAUAGACGUGAUCUACGUGGAAU

TSP-1 3'-UTR 5' ... UAUUUUUAUAUAAUGUUUGCACCA. . . hsa-miR-19a $33^{\prime}$ AGUCAAAACGUAUCUAAACGUGU

TSP-1 3'-UTR 5' ... UAUUUUUAUAUAAUGUUUGCACA. .. hsa-miR-19b 3 ' AGUCAAAACGUACCUÁÁCGUGU

D

Supplemental figure 1. CTGF and TSP-1 3'-UTRs have conserved miR-18 and miR-19 target sites. (A) Diagram indicating the location of the target sequences of miR-18 and miR-19 in the human CTGF and TSP1 gene. (B) MiRNA-target hybrids of the miR-17 92 cluster members miR-18a, miR-19a, and miR-19b with their target sequences, based on prediction by TargetScan Human Release 5.1 (www.targetscan.org). (C) Alignment of the target sites for miR-18 and 19 found in the $3^{\prime}$-UTR region of the CTGF and TSP- 1 gene of human, mouse and rat, illustrating that these sites are highly conserved among species. The 3'UTR region of the TSP-1 gene is not completely annotated in rat, and therefore the miR-19 target site in the rat TSP-1 gene is currently unknown. (D) Sequence alignment of the mature forms of miR-18a, miR-19a, and miR-19b shows that each of these miRNAs are identical in human, mouse and rat. MiR-19a and -b only differ in one nucleotide outside the seed sequence. 
CTGF
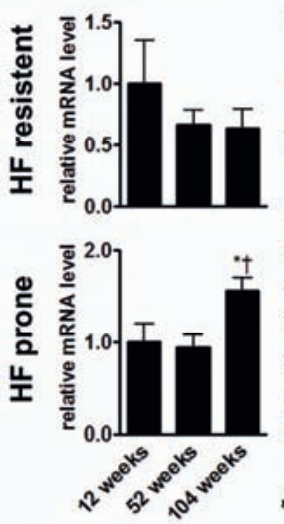

TSP-1
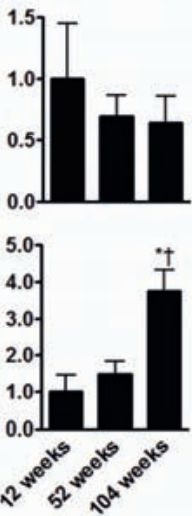

Supplemental figure 2. CTGF and TSP-1 transcript levels in HF resistant and $\mathrm{HF}$ prone mice. CTGF and TSP-1 transcript levels were determined in aged $\mathrm{HF}$ resistant (12 weeks, $\mathrm{n}=8 ; 52$ weeks, $n=8$; and 104 weeks, $n=9)$ and HF prone mice (12 weeks, $n=6$; 52 weeks, $n=11$; and 104 weeks, $n=9)$. RT-PCR analysis showed enhanced CTGF and TSP-1 mRNA levels in the hearts of 104 weeks old HF prone mice, but not in HF resistant hearts. All data were normalized for GAPDH expression and presented as mean \pm SEM. ${ }^{*} P \leq 0.05$ versus 12 weeks of age; $+P \leq 0.05$ versus 52 weeks of age.

\section{A}

Cardiomyocyte

\section{CTGF}

\section{TSP-1}
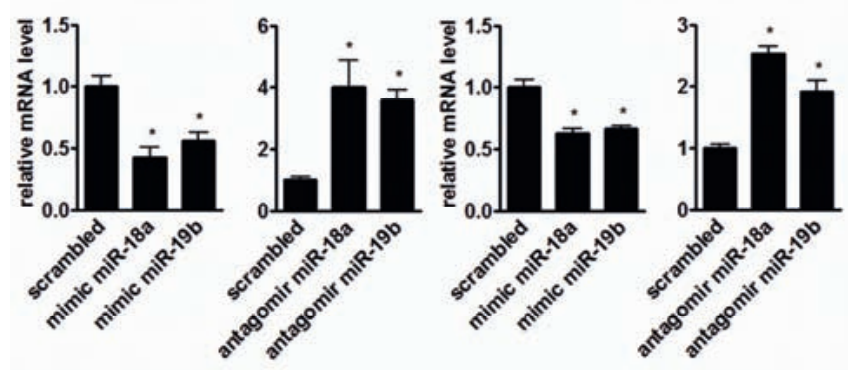

B

Fibroblast

CTGF

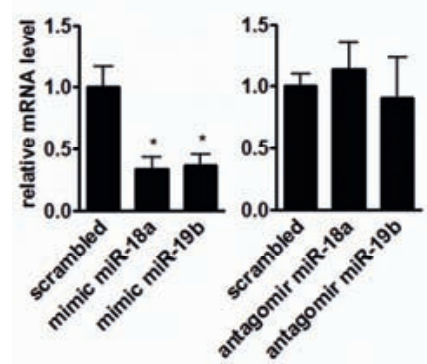

TSP-1

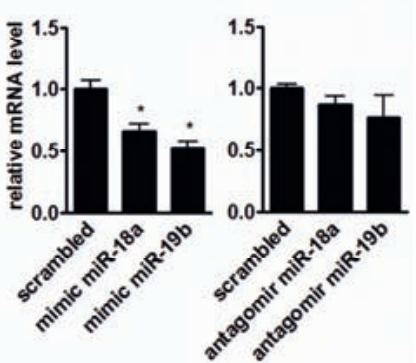

Supplemental figure 3. CTGF and TSP-1 transcripts are regulated by miR-18a and miR-19b in cardiomyocytes. Transcript levels of CTGF and TSP-1 were determined in NRCMs and NRCFs after transfection with a miRNA-specific mimic or antagomir to overexpress or inhibit, respectively, the function of miR-18a and miR-19b. (A) RT-PCR analysis revealed that overexpression of miR-18a and miR-19b in cardiomyocytes significantly repressed CTGF and TSP-1 transcription, while inhibition of these miRNAs enhanced CTGF and TSP-1 mRNA levels. (B) Although overexpression of miR-18a and miR-19b decreased CTGF and TSP-1 expression, blunting of the miRNAs using antagomirs was not sufficient to increase CTGF and TSP-1 levels in cardiac fibroblasts. Mimic and antagomir experiments were performed with $n=4$ per group and data were normalized for GAPDH expression. Data were presented as mean \pm SEM. 


\section{Supplemental references}

1 De Windt LJ, Willemsen PH, Popping S, Van der Vusse GJ, Reneman RS, Van Bilsen M. Cloning and cellular distribution of a group II phospholipase A2 expressed in the heart. J Mol Cell Cardiol. 1997;29(8):2095-2106.

2 Moura R, Tjwa M, Vandervoort P, Van Kerckhoven S, Holvoet P, Hoylaerts MF. Thrombospondin-1 deficiency accelerates atherosclerotic plaque maturation in ApoE-/- mice. Circ Res. 2008;103(10):11811189.

3 Nuovo GJ. In situ detection of microRNAs in paraffin embedded, formalin fixed tissues and the colocalization of their putative targets. Methods. 2010;52(4):307-315. 


\section{Chapter 6}

\section{Matricellular proteins and matrix} metalloproteinases mark the inflammatory and fibrotic response in human cardiac allograft rejection

Davy Vanhoutte, Geert C. van Almen, Johan Van Cleemput, Walter Droogné, Frans Van de Werf, Peter Carmeliet, Johan Vanhaecke, Stephane Heymans 


\begin{abstract}
Background: The cardiac extracellular matrix is involved in cardiac inflammation, remodeling and function of the heart. Whether matrix alterations correlate with degree of inflammation, fibrosis and overall rejection in the human transplanted heart remained, until now, unknown.
\end{abstract}

Methods and Results: Therefore, the expression of matricellular proteins, proteoglycans and metalloproteinases (MMPs) and their inhibitors (TIMPs) were investigated in serial endomyocardial biopsies $(n=102)$, in a cohort of 39 patients within the first year after cardiac transplantation. Out of 14 matrix-related proteins, intragraft transcript and protein levels of syndecan-1 (Synd-1) and MMP-9 showed a strong association with the degree of cardiac allograft rejection (CAR), and an even stronger correlation with the expression of pro-inflammatory cytokines TNF- $\alpha$, IL- 6 and TGF- $\beta$, and with infiltrating $\mathrm{CD}^{+} \mathrm{T}$-cells and $\mathrm{CD} 8^{+}$monocytes. Collagen type la1 and Illa1 transcript levels increased with the degree of CAR, and related to the expression of SPARC, CTGF, TSP-1 and -2, TIMP-1, Testican-1, Synd-1, MMP-9 and MMP-2. Furthermore, a strong correlation between collagens and the number of T-cells and monocytes as well as IL-6 and TGF- $\beta$ transcript levels, indicated the involvement of inflammation in stimulating collagen expression in the transplanted heart.

Conclusion: Intragraft expression of Synd-1 and MMP-9 mark the degree of inflammation, whereas multiple inflammatory markers, non-structural matrix proteins, MMPs and TIMPs closely associate with collagen expression in the transplanted heart. These findings are likely to be of clinical value and deserve further investigation as potential diagnostic and therapeutic targets to prevent human CAR. 


\section{Introduction}

Cardiac allograft rejection (CAR) is characterized by severe cardiac inflammation, cardiomyocyte damage, fibrosis and progressive ventricular dysfunction of the transplanted heart. ${ }^{1}$ Although the one-year survival rate now approaches eighty seven percent, CAR still accounts for most of the early and late deaths after heart transplantation (HTx). ${ }^{1,2}$ The current standard to screen for CAR is the histopathological detection of inflammatory infiltrates and cardiomyocyte necrosis in serial endomyocardial biopsies (EMBs), grading according to the guidelines of the International Society of Heart and Lung Transplantation (ISHLT). ${ }^{1}$ Nevertheless, a more in-depth understanding of the biological and molecular changes associated with CAR is mandatory to develop novel diagnostic and therapeutic strategies.

During the last decade, it has become clear that dynamic changes in the cardiac $\mathrm{ECM}$, including altered expression and/or activity of matricellular proteins, proteoglycans and the matrix metalloproteinase (MMP)-system, does not only determine the mechanical properties of the heart, but also modulates key inflammatory and reparative pathways in animal models of cardiac ischemia ${ }^{3,4}$, increased loading ${ }^{5}$, age-related cardiomyopathy ${ }^{6}$ and/or viral myocarditis. ${ }^{7}$ Reports from several groups advocate a pivotal role for matricellular proteins and proteoglycans, including, thrombospondin (TSP)-1, ${ }^{8,9}$ and- $2,{ }^{9,10}$ connected tissue growth factor (CTGF) ${ }^{11}$, secreted protein, acidic and rich in cystein (SPARC) ${ }^{12}$, syndecan (synd) $-1^{13}$ and $-4^{14}$ and testican- 1 in inflammatory processes. ${ }^{3,15,16}$ Also altered levels of MMP and their specific tissue inhibitors (TIMPs) regulate cardiac ECM remodeling, thereby determining the structural and functional outcome of diseased hearts. ${ }^{15,17}$ MMPs degrade the ECM, which results in matrix fragments that exert potent pro-inflammatory actions. In addition, they process cytokines and chemokines, thereby altering their biological activity. Studies in MMP-2 and -9 deficient mice revealed that both gelatinases have the ability to influence graft survival by modulating T-cell alloreactivity in a model of acute CAR ${ }^{18}$, whereas the use of broad-spectrum MMP-inhibitors significantly enhanced graft survival by suppressing the inflammatory response. ${ }^{19,20}$

Despite their implication in the inflammatory and reparative pathways of the heart, evaluation of matricellular proteins, proteogelycans, MMPs and TIMPs in relation to inflammation, fibrosis and the degree of human cardiac allograft rejection are scarce and fragmentary, and mainly studied MMPs. ${ }^{21,22}$ In the present study, we focused on the intragraft transcript, protein and/or activity levels of matricellular proteins (TSP-1 and - 2, CTGF and SPARC), proteoglycans (synd-1 and -4, Testican-1), MMPs (MMP-1, -2, -9) and TIMPs (TIMP-1 to -4) in relation to (i) the degree of cardiac allograft rejection as measured by ISHLT-grading ${ }^{1}$; (ii) the expression of proinflammatory cytokines TNF- $\alpha$, IL- 6 and TGF- $\beta$; (iii) the influx of $\mathrm{CD}^{+}{ }^{+}$-cells and CD68 ${ }^{+}$ monocytes; and (iv) collagen expression within the first year after heart transplantation. 


\section{Methods}

An expanded methods section is provided in the Supplemental material (pag. 153).

\section{Study design}

EMB and plasma samples were collected from 39 patients, in the first year after cardiac transplantation (Supplemental table 1). All patients were transplanted in Leuven (Belgium) and received standard triple-immunosuppresive therapy, including tacrolimus (Prograft ${ }^{\circledR}$ ), mycophenolate mofetil (CellCept ${ }^{\circledR}$ ) and methylprednisolone (SoluMedrol $^{\circledR}$, Medrol $^{\circ}$ ). Episodes of moderate or severe rejection were treated with increased doses of methylprednisolone. The study was approved by the medical ethical commission of the KUL, and all patients gave written informed consent. This study is consistent with the Declaration of Helsinki.

Protocol EMBs and plasma samples were collected simultaneously during routine controls after cardiac transplantation to rule out rejection. During each catheterization, a minimum of three EMBs were taken and bouin-fixed for routine histology, each of which contained at least $50 \%$ myocardium to exclude a previous biopsy site or scar. ${ }^{1}$ Additional biopsy specimens were immediately frozen in liquid $N_{2}$, then stored in -80 ${ }^{\circ} \mathrm{C}$ until subsequent analysis. Peripheral blood samples were obtained from a central venous sheath and subsequently processed to obtain blood plasma, using plasmaseparating tubes (BD Vacutainer Systems, plymouth, UK). The tubes were centrifuged at $3000 \mathrm{rpm}$ for 15 minutes, aliquoted and stored in $-80^{\circ} \mathrm{C}$ for subsequent analysis.

Biopsy specimens were graded for cellular rejection by a pathologist following the guidelines of the ISHLT. More specifically, we used the adapted 1990 grading system with omission of grade 2, which was commonly used before the implementation of the current 2005 adaptation. ${ }^{1}$ Of 102 EMB samples, 37 percent showed no histological signs of allograft rejection (grade 0 ) and 30 percent revealed minimal evidence of allograft rejection (grade $1 \mathrm{~A}$ ). A diffuse inflammatory infiltrate, but without cardiomyocyte damage, was found in 25 percent of the EMBs (grade 1B). Eight percent of all biopsies indicated severe allograft rejection by multifocal or diffuse infiltration of inflammatory cells with myocyte damage (grade 3 ). Thus the frequency of rejection episodes requiring treatment was low in the present study population. For the current analysis case samples ("rejection") were selected on basis of the presence of biopsyproven ISHLT-grade $>0 .{ }^{1}$ These samples were compared with specimens classified as grade 0 , showing no clinically significant rejection.

\section{Real-time polymerase chain reaction, histology, ELISA and In situ gelatin zymography}

The obtained EMBs and plasma specimens were subsequently prepared for further molecular and histological analysis, including, RNA isolation and real-time polymerase chain reaction (RT-PCR), immunohistochemical analysis, in situ gelatin zymography and 
enzyme linked immunosorbent assay (ELISA). Experimental materials and methods are described extensively in the Data Supplement.

\section{Statistical analysis}

Analyses were performed with the SPSS 11.0 statistical software package (Mac OsX; SPSS; Chicago, IL, USA) and two-sided $\mathrm{P}<0.05$ was considered statistically significant. Data are expressed as mean \pm SEM. All continuous variables were tested for normality using the method of Kolmogorov and Smirnov. Subsequently, mRNA and protein expression levels were compared by Kruskal-Wallis test in combination with a Dunn posthoc analysis to correct for multiple comparison or by one-way ANOVA with Bonferroni post-hoc analysis when appropriate. An additional ANOVA with post hoc analysis for linearity was used to define the relation between the ISHLT-rejection grade $(=X)$ and the expression levels $(=Y)$. The linearity of the data is indicated by the $R^{2}$-value, and was considered statistically significant when $p<0.05$. Logarithmic transformation was performed when data deviated substantially from linearity. Standard Pearson or nonparametric Spearman correlations, indicated as $r$, were used to assess the relationship between the mRNA expression levels, protein expression levels and plasma levels depending on respectively Gaussian or non-Gaussian distribution of the 2 groups.

\section{Results}

Intragraft synd-1, MMP-9, and TIMP-1 transcript levels relate to the degree of CAR

A total of 102 EMBs from our cohort of $39 \mathrm{HTx}$ patients were examined by real-time quantitative $\mathrm{PCR}$ for the differential $\mathrm{mRNA}$ expression of several matricellular proteins and proteoglycans (TSP-1 and -2, CTGF, SPARC, synd-1 and -4 , and testican-1) and MMPs/TIMPs (MMP-1, -2 and -9 and TIMP-1 to -4 ) in relation to the different ISHLT rejection grades (Table 1 ).

Normalized intragraft transcript levels of synd-1, MMP-9 and TIMP-1 showed a significant and positive correlation with the degree of CAR (Table 1). Whereas no major differences were seen between grade 0 and grade $1 A$, synd-1, MMP-9 and TIMP-1, respectively, showed a 1.8-, 3.1- and 1.8-fold increase in grade $1 B(p<0.001)$ and a $37.2-, 9.4-$ and 7.4-fold increase in grade 3 ( $p<0.001$ ), as compared to grade 0 EMBs (Table 1). In contrast, only a moderate association was observed for TSP-2, CTGF, TIMP-2, -3 and -4 with the degree of CAR; and no correlation was seen for Synd-4, SPARC, testican-1 and MMP-2 (Table 1).

Taken together, EMB transcript levels of synd-1, MMP-9 and TIMP-1 strongly relate to the degree of CAR. 
Table 1. Intragraft transcript levels in relation to rejection grades on EMB

\begin{tabular}{|c|c|c|c|c|c|c|}
\hline & \multirow[t]{2}{*}{$\begin{array}{l}\text { Grade } 0 \\
(n=38)\end{array}$} & \multirow[t]{2}{*}{$\begin{array}{l}\text { Grade } 1 A \\
(n=31)\end{array}$} & \multirow[t]{2}{*}{$\begin{array}{l}\text { Grade 1B } \\
(n=25)\end{array}$} & \multirow[t]{2}{*}{$\begin{array}{c}\text { Grade } 3 \\
(n=8)\end{array}$} & \multicolumn{2}{|c|}{$\begin{array}{l}\text { ANOVA post test for } \\
\text { linearity }\end{array}$} \\
\hline & & & & & R2-value & $\mathrm{P}$-value \\
\hline \multicolumn{7}{|c|}{ Matrix glycoproteins } \\
\hline Synd-1 & $0.4 \pm 0.1$ & $0.6 \pm 0.1$ & $0.7 \pm 0.1$ & $14.9 \pm 8.0^{*+\ddagger}$ & 0.286 & $P<0.0001$ \\
\hline Synd-4 & $48 \pm 4$ & $42 \pm 4$ & $49 \pm 5$ & $43 \pm 7$ & 0.001 & n.s. \\
\hline TSP-1 & $210 \pm 27$ & $181 \pm 21$ & $234 \pm 22$ & $297 \pm 64$ & 0.034 & n.s. \\
\hline TSP-2 & $812 \pm 103$ & $960 \pm 180$ & $1194 \pm 262$ & $1894 \pm 559^{*}$ & 0.107 & $P<0.01$ \\
\hline CTGF & $0.6 \pm 0.2$ & $0.4 \pm 0.1$ & $0.6 \pm 0.2$ & $1.3 \pm 0.5+$ & 0.069 & $P<0.05$ \\
\hline SPARC & $10.4 \pm 2.7$ & $11.0 \pm 2.6$ & $12.4 \pm 1.6$ & $17.5 \pm 4.6$ & 0.018 & n.s. \\
\hline Testican-1 & $19.0 \pm 2.5$ & $16.4 \pm 3.3$ & $17.9 \pm 3.2$ & $22.4 \pm 3.6$ & 0.006 & n.s \\
\hline \multicolumn{7}{|c|}{ MMPs and TIMPs } \\
\hline MMP1 & $2.2 \pm 0.3$ & $3.0 \pm 0.5$ & $5.4 \pm 0.7^{* \ddagger}$ & $5.0 \pm 1.0^{*}$ & 0.118 & $P<0.01$ \\
\hline MMP2 & $19.8 \pm 2.0$ & $22.4 \pm 2.8$ & $22.2 \pm 1.9$ & $24.4 \pm 2.7$ & 0.001 & n.s. \\
\hline MMP9 & $2.7 \pm 0.6$ & $3.0 \pm 0.7$ & $8.4 \pm 2.0^{*+}$ & $25.5 \pm 7.0^{*+\ddagger}$ & 0.520 & $P<0.0001$ \\
\hline TIMP1 & $58 \pm 7$ & $58 \pm 7$ & $104 \pm 14^{*+}$ & $429 \pm 176^{*+ \pm}$ & 0.479 & $P<0.0001$ \\
\hline TIMP2 & $112 \pm 6$ & $105 \pm 7$ & $116 \pm 6$ & $136 \pm 22$ & 0.046 & $P<0.05$ \\
\hline TIMP3 & $0.7 \pm 0.1$ & $0.5 \pm 0.1$ & $0.9 \pm 0.2$ & $1.8 \pm 0.2^{*+}$ & 0.267 & $P<0.01$ \\
\hline TIMP4 & $0.10 \pm 0.01$ & $0.06 \pm 0.01$ & $0.11 \pm 0.06$ & $0.31 \pm 0.06^{*+}$ & 0.167 & $P<0.05$ \\
\hline
\end{tabular}

ISHLT-grade indicates the histopathological grade of cardiac allograft rejection as set by the International Society of Heart and Lung Transplantation. Transcript levels are given in arbitrary units relative to GAPDH expression and presented as mean \pm SEM. ${ }^{*}, P<0.05$ versus grade $0 ;{ }^{\dagger}, P<0.05$ versus grade $1 \mathrm{~A}^{\ddagger}, P<0.05$ versus grade $1 \mathrm{~B}$

EMB indicates endomyocardial biopsy; Synd-1, Syndecan-1; TSP, thrombospondin ; CTGF, connective tissue growth factor; SPARC, secreted protein acidic and rich in cysteine; MMP, matrix metalloproteinase; TIMP, tissue inhibitor of matrix metalloproteinases.

Transcript levels of Synd-1, MMP-9, CTGF, SPARC and TIMP-1 relate to IL-6, TNF- $\alpha$, and/or TGF-B

Pro-inflammatory cytokines, including interleukin-6 (IL-6), tumor necrosis factor- $\alpha$ (TNF- $\alpha$ ) and transforming growth factor- $\beta$ (TGF- $\beta$ ) function as potent chemoattractants, and provide essential signals for the dynamic trafficking of T-cells and monocytes into allografts. ${ }^{23-25}$ In addition, both IL-6 and TGF- $\beta$ negatively regulate cardiac allograft survival through their pro-fibrotic effects. ${ }^{23}$ Concordant, we next investigated the intragraft transcript levels of the matricellular proteins, proteoglycans and MMPs/TIMPs intragraft in relation to the transcript levels of IL-6, TNF- $\alpha$, and TGF- $\beta$ (Figure 1 and supplemental figure 1). 
A

IL-6 mRNA

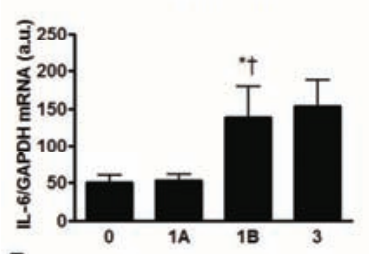

$\log (\mathrm{IL}-6 \mathrm{mRNA})$

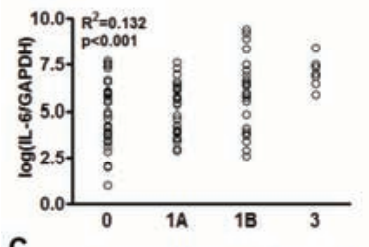

C

synd-1 mRNA vs IL-6 mRNA

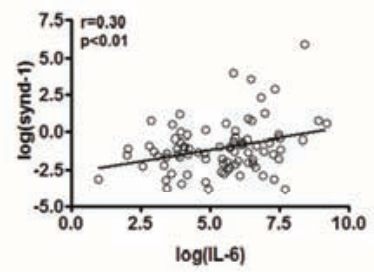

D

CTGF MRNA vs IL-6 IRNA

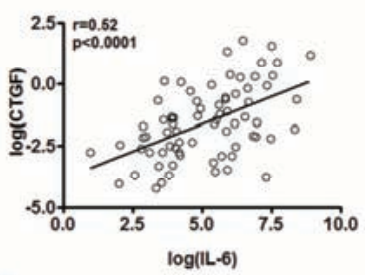

E

SPARC MRNA VS IL.6 MRNA

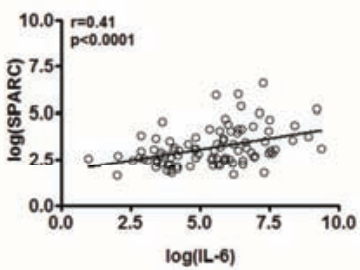

TNF- $\alpha$ mRNA

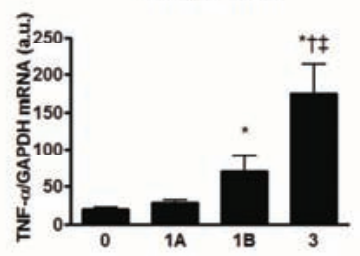

$\log (\mathrm{TNF}-\alpha \operatorname{mRNA})$

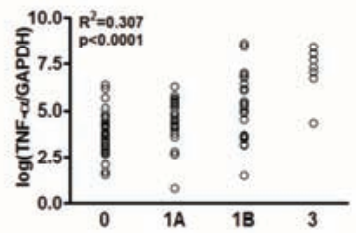

synd-1 mRNA vs TNF- $\alpha$ mRNA

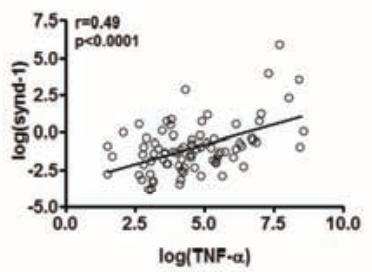

CTGF MRNA vs TNF- $\alpha$ mRNA

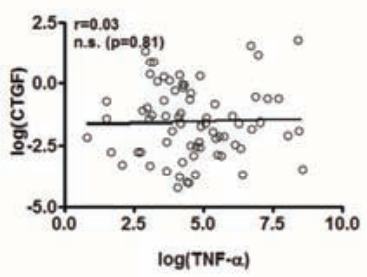

SPARC MRNA vs TNF- $\alpha$ mRNA

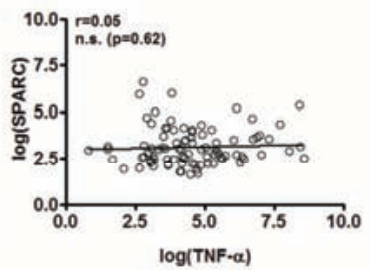

TGF- $\beta$ mRNA

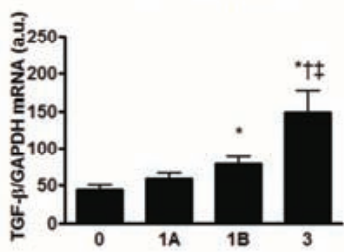

$\log ($ TGF- $\beta$ mRNA)

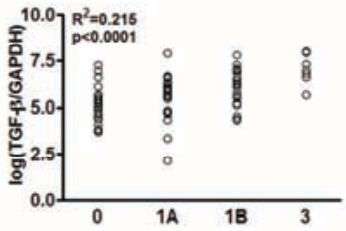

synd-1 mRNA vs TGF- $\beta$ mRNA

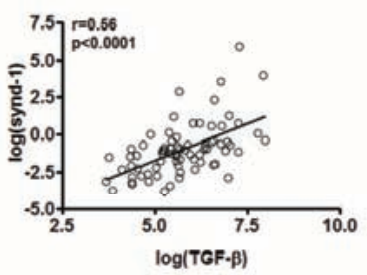

CTGF MRNA vs TGF- $\beta$ MRNA

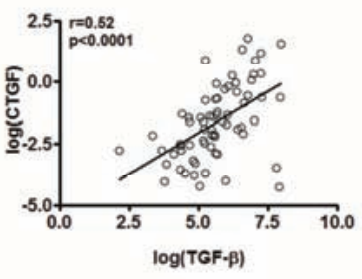

SPARC MRNA VS TGF- $\beta$ MRNA

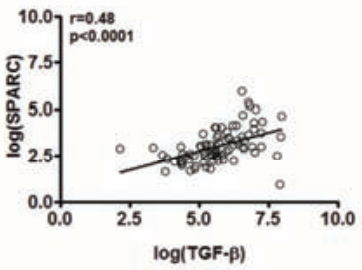

Figure 1A-E. Intragraft transcript levels of in relation to the expression of pro-inflammatory cytokines. Figure $1 \mathrm{~F}-\mathrm{H}$ continued on page 138 
F

MMP-1 MRNA vs IL-6 MRNA

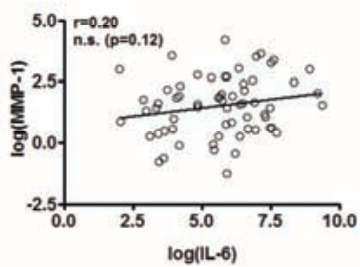

G

MMP-9 MRNA vs IL-6 mRNA

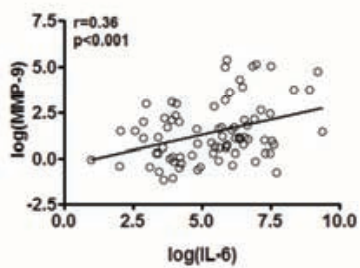

H

TIMP-1 MRNA vs IL-6 MRNA

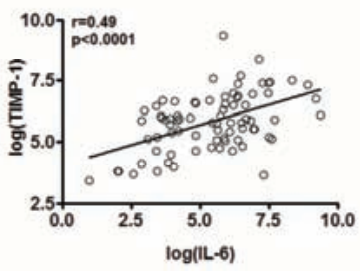

MMP-1 mRNA vs TNF- $\alpha$ mRNA

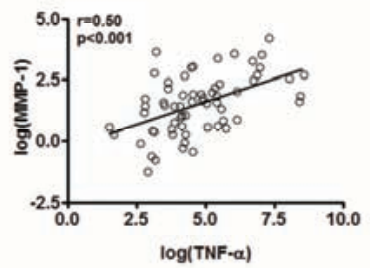

MMP-9 mRNA vs TNF- $\alpha$ mRNA

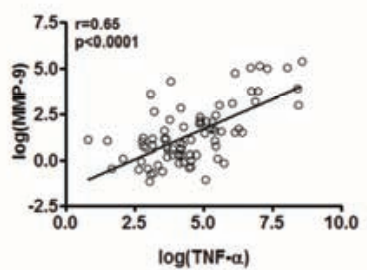

TIMP-1 mRNA vs TNF- $\alpha$ mRNA

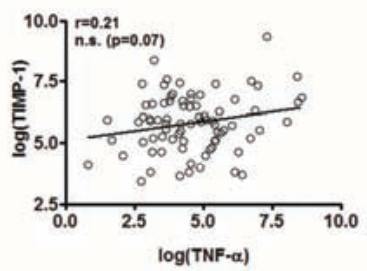

MMP-1 MRNA vs TGF- $\beta$ MRNA

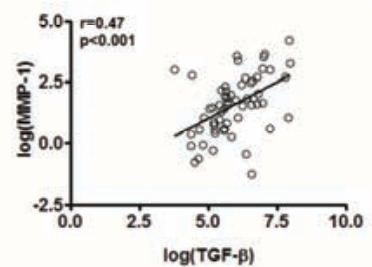

MMP-9 MRNA vs TGF- $\beta$ mRNA

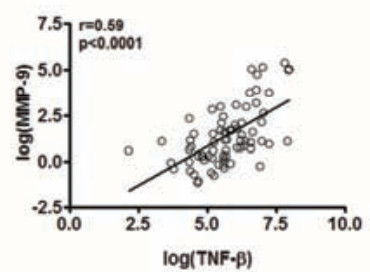

TIMP-1 MRNA vs TGF- $\beta$ MRNA

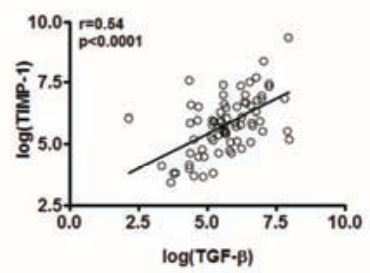

Figure 1. Intragraft transcript levels of in relation to the expression of pro-inflammatory cytokines. (A-B) Intragraft expression levels of IL-6, TNF- $\alpha$, and TGF- $\beta$ progressively increase with and significantly relate to the ISHLT grade of CAR. (C-H) Intragraft transcript levels of (C) Syndecan-1, (D) CTGF, (E) SPARC, (F) MMP-1, (G) MMP-9 and (H) TIMP-1 in relation to the mRNA expression levels of IL-6, TNF- $\alpha$, and TGF- $\beta .^{*}, P<0.05$ versus. grade $0 ; \dagger, P<0.05$ versus. grade $1 \mathrm{~A} ; \ddagger, P<0.05$ versus. grade $1 \mathrm{~B} ; \mathrm{n}=102$, including $\mathrm{n}=38$ for grade 0 , $\mathrm{n}=31$ for grade $1 \mathrm{~A}, \mathrm{n}=25$ for grade $1 \mathrm{~B}$ and $\mathrm{n}=8$ for grade 3 .

Transcript levels of IL-6, TNF- $\alpha$, and TGF- $\beta$ significantly increased in grade $1 \mathrm{~B}$ and 3 rejecting EMBs and significantly relate to the degree of CAR (Figure 1A-B). Out of 14 matrix proteins, intragraft mRNA expression of synd-1 and MMP-9 revealed the strongest correlations with the expression levels of IL-6, TNF- $\alpha$, and TGF- $\beta$ (Figure 1C and G). For TIMP-1, CTGF and SPARC, all implicated in stimulating cardiac fibrosis ${ }^{16,17}$, a strong correlation was found with IL- 6 and TGF- $\beta$, but not with TNF- $\alpha$ (Figure 1D-E and H). Markedly, Synd-1, CTGF, SPARC, MMP-1 and -9 and TIMP-1 correlated stronger with the expression levels of IL-6, TNF- $\alpha$, and/or TGF- $\beta$ as compared to their relation with the degree of CAR (Table 1). Only moderate or no correlations were noted for the other proteins included in this study (Supplemental figure 1).

Together, these data suggest that intragraft expression of Synd-1, MMP-9 and TIMP-1, but also MMP-1, CTGF and SPARC strongly mirrors the expression of proinflammatory cytokines IL-6, TNF- $\alpha$, and/or TGF- $\beta$ during human CAR. 
Transcription of synd-1 and MMP-9, but also CTGF, SPARC, TSP-1 and -2 coincide with the influx of T-cells and monocytes in the transplanted heart

Inflammatory infiltrates associated with CAR, largely consist out of T-cells and monocytes. $^{1}$ Next, a total of 71, randomly chosen, EMBs were examined by immunohistochemistry to evaluate the influx of $\mathrm{CD}^{+}$T-cells and $\mathrm{CD} 68^{+}$monocytes. Both $\mathrm{CD}^{+}{ }^{+} \mathrm{T}$ cells and $\mathrm{CD} 68^{+}$monocytes infiltrates in these biopsies revealed a strong association with the presence of rejection (Figure $2 \mathrm{~A}-\mathrm{B}$ ). Whereas only few $\mathrm{CD}^{+}{ }^{+}$-cells and $\mathrm{CD} 68^{+}$ monocytes were present in grade $0 \mathrm{EMBs}$, increasing number of infiltrates were observed with increasing grade of cardiac allograft rejection (Figure $2 \mathrm{~A}-\mathrm{C}$ ). $\mathrm{CD}^{+}-$and $\mathrm{CD}^{+} 8^{+}$-infiltrates were especially present in $\mathrm{EMBs}$ displaying a severe rejection episode accompanied by myocyte necrosis, grade 3 (Figure $3 C$ ). Transcript levels of the proinflammatory cytokines IL-6, TNF- $\alpha$, and TGF- $\beta$ were highly associated with the influx of $\mathrm{CD}^{+} \mathrm{T}$-cells and $\mathrm{CD}^{+} 8^{+}$monocytes (Figure 2D-F), indicating a pivotal role for these cytokines in the inflammatory pathways underlying human CAR. More importantly, the influx of $\mathrm{CD}^{+}$T-cells and $\mathrm{CD} 68^{+}$monocytes strongly related to the transcription of respectively synd-1, MMP-9, CTGF, SPARC, TSP-2 and -1 (from highest to lowest; Figure 2G-L), but not to that of TIMP-1 (Figure 2M). Similar to their relation with IL-6, TNF- $\alpha$, and/or TGF- $\beta$, the correlations noted with infiltrated $\mathrm{CD}^{+}{ }^{+}$-cells and $\mathrm{CD}^{-} 8^{+}$monocytes were stronger as compared to their relation with the grade of CAR (Table 1). No significant correlations were found for the remaining proteins included in this study (Supplemental figure 2).

Together, these data reveal a close relationship between inflammation and the induction of synd-1, MMP-9, CTGF, SPARC, TSP-2 and -1 in the transplanted hearts. 
A

CD3* T-cells

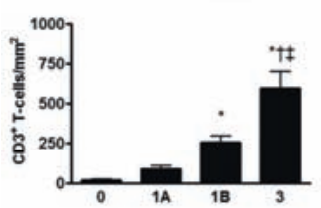

$\log \left(\mathrm{CD}^{*} \mathrm{~T}\right.$-cells $)$

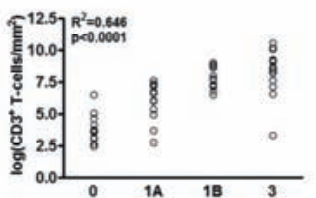

B

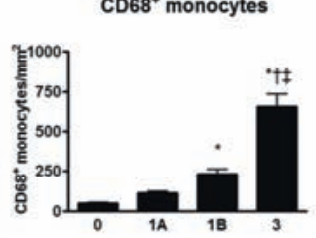

grade 1B

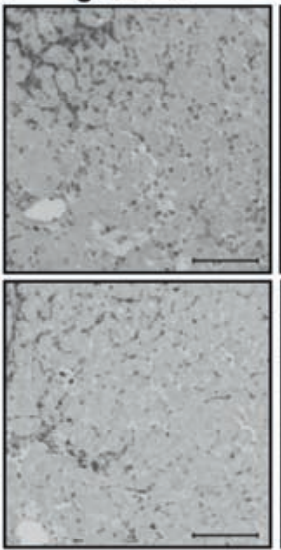

E

TNF- $\alpha$ mRNA vs $\mathrm{CD3}^{+} \mathrm{T}$-cells

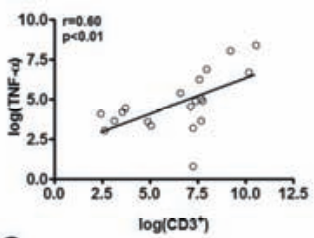

G

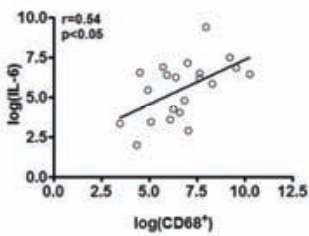

TGF- $\beta$ mRNA vs $\mathrm{CD}^{\circ} 8^{*}$ monocytes
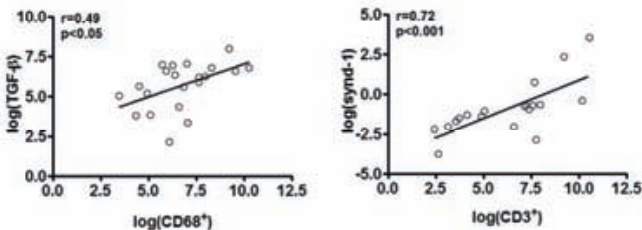

I

TSP-2 mRNA vs $\mathrm{CD}^{*}$ T-cells

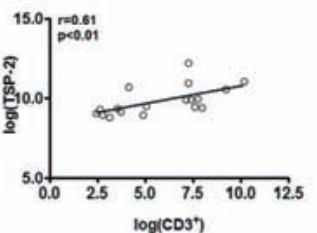

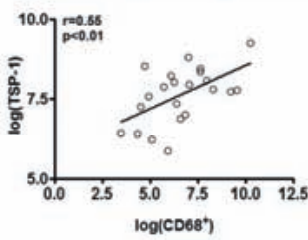

$\log \left(\mathrm{CD}_{68}{ }^{*}\right.$ monocytes $)$

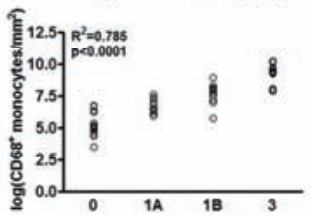

grade 3

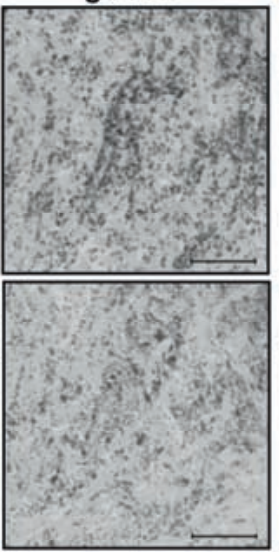

F

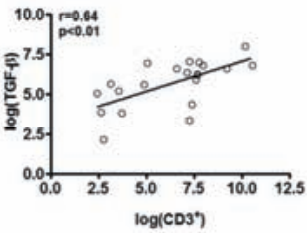

H TSP-1 mRNA vs $\mathrm{CD}^{*}$ T-cells

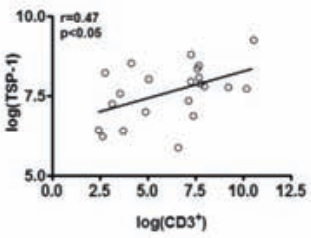

TNF- $\alpha$ mRNA vs CD68 ${ }^{*}$ monocytes

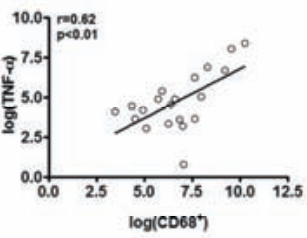

synd-1 mRNA vs $\mathrm{CD}^{\circ} 8^{*}$ monocytes

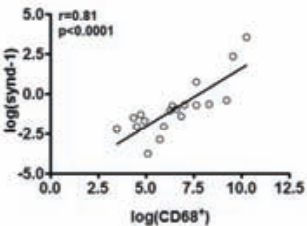

TSP-2 mRNA vs CD68* monocytes

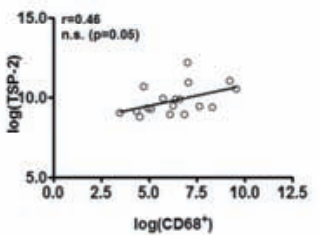

Figure 2A-I. Transcription of synd-1, TSP-1 and -2, CTGF, SPARC and MMP-9 coincide with the influx of Tcells and monocytes in the transplanted heart. Figure 2J-M continued on page 141 
K

CTGF MRNA vs $\mathrm{CD}^{*}$ T-cells CTGF MRNA vs $\mathrm{CD}^{+} 8^{*}$ monocytes

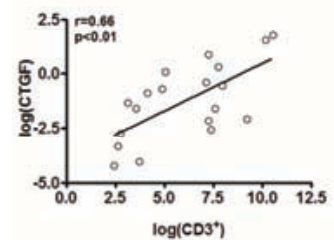

$\mathbf{L}$

MMP-9 mRNA vs $\mathrm{CD}^{*}$ T-cells

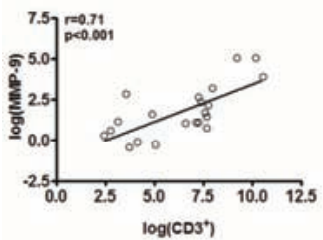

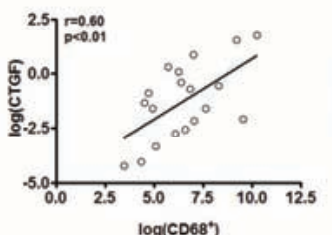

$\log \left(\mathrm{CD}^{\circ} 8^{4}\right)$

MMP-9 mRNA vs $\mathrm{CD}^{\circ} 8^{*}$ monocytes

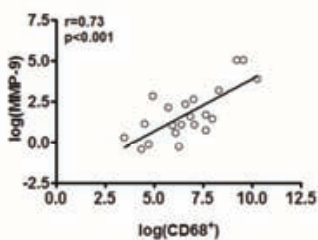

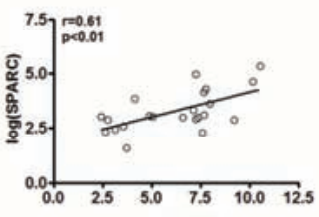

M

TIMP. 1 mRNA vs $\mathrm{CD}^{*}$ T-cells

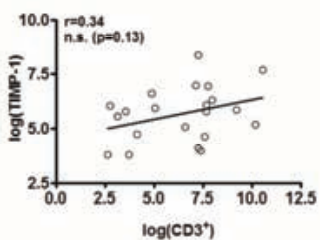

SPARC mRNA vs CD68* monocytes

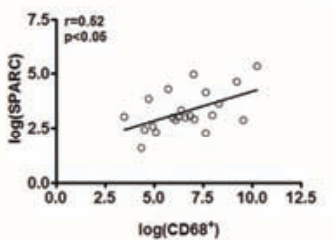

TIMP-1 mRNA vs CD68* monocytes

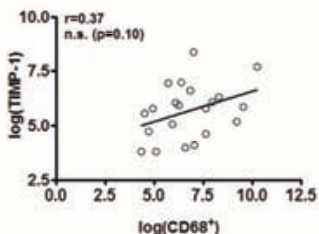

Figure 2. Transcription of synd-1, TSP-1 and -2, CTGF, SPARC and MMP-9 coincide with the influx of T-cells and monocytes in the transplanted heart. Immunohistochemical analysis revealed a significantly increased influx of (A) $\mathrm{CD}^{+}$T-cells and (B) $\mathrm{CD}^{+} 8^{+}$monocytes with increasing grade of human CAR. (C) Representative microphotographs of CD3 and CD68 immunoreactivity in EMBs displaying CAR grade 0,1A, 1B and 3. Scalebar: $100 \mu \mathrm{m}$. Transcript levels of (D) IL-6, (E) TNF- $\alpha$, (F) TGF- $\beta$, (G) Synd-1, (H) TSP-1, (I) TSP-2, (J) CTGF, (K) SPARC, (L) MMP-9 and (M) TIMP-1 in relation to the influx of $\mathrm{CD}^{+}{ }^{+}$T-cells and CD68 ${ }^{+}$monocytes. ${ }^{*}, P<0.05$ versus. grade $0 ;+, P<0.05$ versus. grade $1 A ; \ddagger, P<0.05$ versus. grade $1 B ; n=64$, including $n=21$ for ISHLT grade $0, n=19$ for grade $1 A, n=16$ for grade $1 B$ and $n=8$ for grade 3 .

Matricellular proteins, proteoglycans, MMPs, TIMPs and cardiac allograft fibrosis

Besides inflammation, the majority of these macromolecules have been reported to influence the expression, deposition, assembly and/or maturation of the newly deposited extracellular collagen matrix in various cardiac pathologies. ${ }^{3,15,16}$ Moreover, cardiac allograft fibrosis, another hallmark of CAR, has been identified as an important limiting factor towards long-term graft survival. ${ }^{23}$ Intragraft transcript levels of COLla1, and COLIIla1 significantly increased with the production of pro-inflammatory/profibrotic cytokines TGF- $\beta$ and IL- 6 , the influx of $\mathrm{CD}^{+}$T-cells and $\mathrm{CD} 68^{+}$monocytes and increasing grade of rejection (Figure 3A-D, Table 2). Together, indicating a close association between T-cells, monocytes and collagen expression during CAR. Interestingly, intragraft transcript levels of SPARC, CTGF, TSP-2, TIMP-1, Testican-1, TSP-1, Synd-1, MMP-9 and MMP-2 (from highest to lowest) all strongly correlated with the expression levels of both COLla1 and COLIIla1 (Table 2). Next, transmural interstitial fibrosis, visualized by sirius red staining, was analyzed in all biopsies. As illustrated in grade 3 EMBs (Figure 3E), collagen deposition was most prominent in areas surrounding cardiomyocytes, indicating replacement fibrosis due to the loss of cardiomyocytes. In some of the cases, fibrosis was also more pronounced in the peri-vascular areas, indicating reactive fibrosis (Figure 3E). Surprisingly, total collagen deposition did not reflect the mRNA 
expression profiles of COLla1 and COLIIla1 (Figure 3F). Whereas the total amount of deposited collagen showed a trend towards increase in grade $1 \mathrm{~A}-1 \mathrm{~B}$ compared to grade 0 , no differences were seen in grade 3 compared to grade 0 EMBs (Figure 3F). Keeping our previous findings in mind, these data suggest that the increased expression of COLla1 and COLIIla1 in grade 3 rejecting EMBs might counterbalance by an extensive MMP-mediated proteolytic degradation. In addition, a significant increase in collagen deposition was noted 8 months after transplantation, as compared to 1 month (Figure 3G).

Table 2. Intragraft transcript levels in relation to collagen synthesis

\begin{tabular}{|c|c|c|c|c|}
\hline & \multicolumn{2}{|c|}{ Col la1 } & \multicolumn{2}{|c|}{ Col Illa1 } \\
\hline & $r$-value & P-value & $r$-value & P-value \\
\hline \multicolumn{5}{|c|}{ Proteoglycans and matricellular proteins } \\
\hline Synd-1 & 0.45 & $<0.0001$ & 0.43 & $<0.0001$ \\
\hline Synd-4 & 0.15 & n.s. & 0.16 & n.s. \\
\hline TSP-1 & 0.57 & $<0.0001$ & 0.54 & $<0.0001$ \\
\hline TSP-2 & 0.76 & $<0.0001$ & 0.72 & $<0.0001$ \\
\hline CTGF & 0.80 & $<0.0001$ & 0.77 & $<0.0001$ \\
\hline SPARC & 0.91 & $<0.0001$ & 0.84 & $<0.0001$ \\
\hline Testican-1 & 0.58 & $<0.0001$ & 0.66 & $<0.0001$ \\
\hline \multicolumn{5}{|c|}{ MMPs and TIMPs } \\
\hline MMP-1 & 0.09 & n.s. & 0.07 & n.s. \\
\hline MMP-2 & 0.40 & $<0.001$ & 0.40 & $<0.001$ \\
\hline MMP-9 & 0.42 & $<0.0001$ & 0.40 & $<0.0001$ \\
\hline TIMP-1 & 0.63 & $<0.0001$ & 0.61 & $<0.0001$ \\
\hline TIMP-2 & 0.27 & $<0.05$ & 0.26 & $<0.05$ \\
\hline TIMP-3 & 0.08 & n.s. & 0.16 & n.s. \\
\hline TIMP-4 & -0.10 & n.s. & -0.04 & n.s. \\
\hline \multicolumn{5}{|c|}{ Pro-inflammatory cytokines } \\
\hline IL-6 & 0.41 & $<0.0001$ & 0.38 & $<0.0001$ \\
\hline TNF- $\alpha$ & 0.12 & n.s. & 0.20 & $<0.05$ \\
\hline TGF- $\beta$ & 0.67 & $<0.0001$ & 0.72 & $<0.0001$ \\
\hline
\end{tabular}

Synd-1 indicates Syndecan-1; TSP, thrombospondin; CTGF, connective tissue growth factor; SPARC, secreted protein acidic and rich in cysteine; MMP, matrix metalloproteinase; TIMP, tissue inhibitor of matrix metalloproteinases; and n.s., not significant. 
A

col1A1 mRNA vs $\mathrm{CD}^{*}$ T-cells

col1A1 mRNA vs CD68+ monocytes
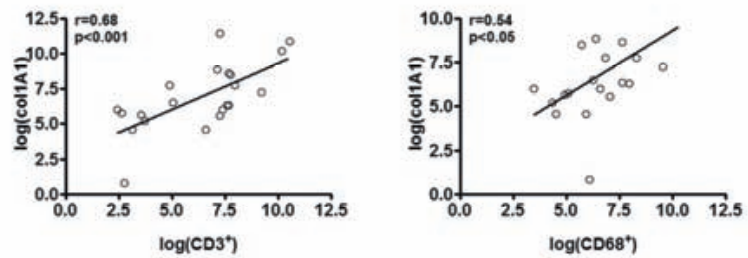

B

col3A1 mRNA vs $\mathrm{CD}^{+}$T-cells

col3A1 mRNA vs $\mathrm{CD}^{+} 8^{+}$monocytes
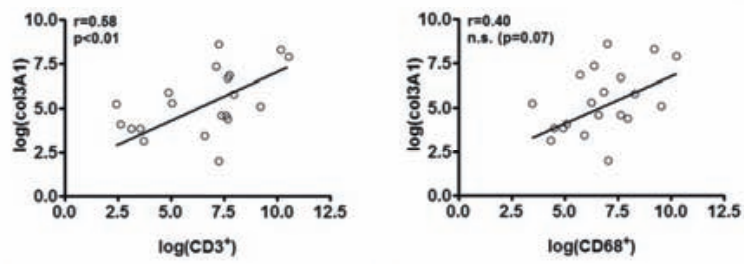

C

Col1A1 mRNA

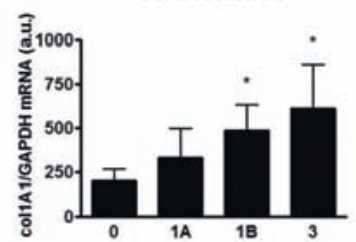

D

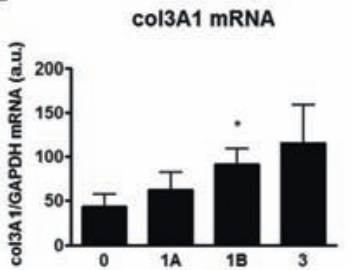

E

Fibrosis, post HTx

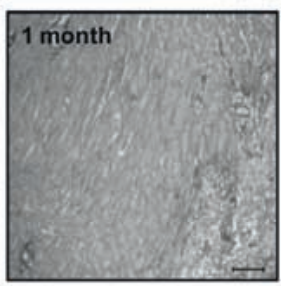

F

Fibrosis

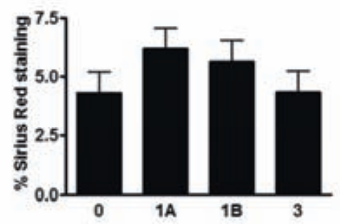

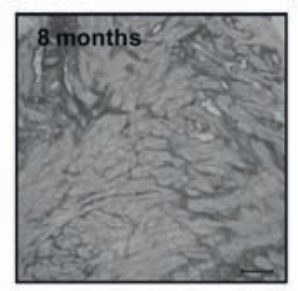

G

Fibrosis, post HTX

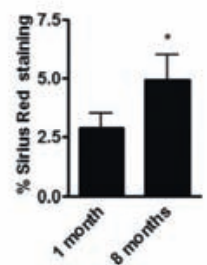

Figure 3. Cardiac allograft fibrosis. Intragraft transcript levels of collagen I type 1 (A) and collagen 1 type III (B) coincide with the influx of $\mathrm{CD}^{+} \mathrm{T}$-cells and $\mathrm{CD}^{+} 8^{+}$monocytes and (C-D) progressively increased with increasing grade of CAR ( $*$ : $<0.01$ compared to grade 0$)$. (E) Repesentatitive sirius red stained grade 3 rejecting EMBs at 1 and 8 months after HTx. Scale-bar: $100 \mu \mathrm{m}$. (F) Sirius red stained collagen deposition with respect to the different grades of CAR. (G) Cardiac allograft fibrosis is significantly increased at 8 months, as compared to 1 month after HTx, as revealed by Sirius red collagen staining. ${ }^{*}$ : $<<0.01$. 
Synd-1 and MMP-9 protein expression mark the influx of T-cells and monocytes during CAR

Encouraged by our previous findings, showing a strong association between increased transcript levels of synd-1 and MMP-9 and the degree of CAR; and an even stronger correlation with the expression of IL-6, TNF- $\alpha$, and TGF- $\beta$, the influx of CD3 ${ }^{+}$T-cells and $\mathrm{CD}^{+} 8^{+}$monocytes and the expression of COLla1 and COLIIla1, we next investigated whether synd-1- and MMP-9-protein expression marked the degree of inflammation in the transplanted hearts.

In agreement with the observed mRNA expression profiles, Synd-1 and MMP-9 protein levels significantly increased with increasing grade of CAR (Table 3, Figure 4A). Strikingly, Synd-1 and MMP-9 immunoreactivity were minimally detectable in grade 0 and $1 \mathrm{~A}$, but progressively increased in $\mathrm{EMBs}$ displaying grade $1 \mathrm{~B}$ or grade 3 rejection episodes. In grade 3 EMBs, a 6.3 fold increase for Synd-1 and a 13.9 fold increase for MMP-9 protein expression was observed, as compared to ISHLT grade 0 ( $p<0.0001$ for both; Table 3). As illustrated in figure 4A, Synd-1 and MMP-9 highly co-localized with infiltrating mononuclear cells and strongly correlated to the degree of infiltrating $\mathrm{CD}^{+}$ T-cells and $\mathrm{CD}^{+} 8^{+}$monocytes (Figure $4 \mathrm{~B}-\mathrm{C}$ ). In addition, a diffuse immunostaining for MMP-9 and Synd-1 was found in the interstitial matrix and in areas with extensive fibrosis (Figure 4A).

Taken together, our data clearly indicate intragraft Synd-1 and MMP-9 protein expression as potent markers for infiltrating T-cells and monocytes in the transplanted heart.

\section{In Situ gelatinolitic activity correlates with cardiac allograft rejection}

Finally, we assessed the intragraft MMP-2 and -9 gelatinolytic activity on cryosections. In situ gelatin zymography revealed a strong positive correlation between MMP2/9gelatinolytic activity and the rejection grade (with an average gelatinolytic activity for grade 0 of: $0.83 \pm 0.31(n=6)$; grade $1 A: 1.0 \pm 0.45(n=5)$; grade $1 B$ : $3.0 \pm 0.26(n=6)$; grade 3: $3.5 \pm 0.29(n=4) ; r=0.824, p<0.0001, n=21$; Figure 5). All of the 11 randomly studied non- and minimal- rejecting EMBs (grade 0 and $1 \mathrm{~A}$ ) scored low and exposed a minimal (score $0 ; n=4$ ), a focal (score $1, n=4$ ), or a moderate (score $2 ; n=3$ ) gelatinolytic activity. For grade 1B, only 1 out of 6 EMBs displayed a moderate gelatinolytic activity, 4 patients a diffuse (score 3 ) and 1 a high (score 4) gelatinolytic activity. Finally, 2 out of 4 grade 3 rejecting patients showed a diffuse and $2 \mathrm{EMBs}$ a high gelatinolytic activity (Figure 5E-H).

Taken together these data suggest that the discrepancy noted between collagen expression and deposition might be the result of the highly enhanced MMPgelatinolytic activity in grade 3 rejecting EMBs. 
Table 3. Synd-1 and MMP-9 protein levels in relation to rejection grades on EMB

\begin{tabular}{|c|c|c|c|c|c|c|}
\hline & \multirow{2}{*}{$\begin{array}{l}\text { Grade } 0 \\
(n=21)\end{array}$} & \multirow{2}{*}{$\begin{array}{l}\text { Grade } 1 A \\
(n=19)\end{array}$} & \multirow{2}{*}{$\begin{array}{l}\text { Grade 1B } \\
(n=16)\end{array}$} & \multirow{2}{*}{$\begin{array}{c}\text { Grade } 3 \\
(n=15)\end{array}$} & \multicolumn{2}{|c|}{ ANOVA post test for linearity } \\
\hline & & & & & $R^{2}$-value & P-value \\
\hline Synd-1, \% & $2.04 \pm 0.14$ & $4.07 \pm 0.23$ & $5.24 \pm 0.39^{*}$ & $12.89 \pm 0.90^{* \neq \ddagger}$ & $R^{2}=0.692$ & $P<0.0001$ \\
\hline MMP-9, \% & $0.12 \pm 0.02$ & $0.35 \pm 0.12$ & $0.79 \pm 0.19^{*}$ & $1.67 \pm 0.60^{*+\ddagger}$ & $R^{2}=0.240$ & $P<0.0001$ \\
\hline
\end{tabular}

ISHLT-grade indicates the histopathological grade of cardiac allograft rejection as set by the International Society of Heart and Lung Transplantation. Protein levels are given as percentage positive staining area relative to total tissue area and presented as mean $\pm \mathrm{SEM}^{*}, P<0.05$ versus grade $0 ;{ }^{+}, P<0.05$ versus grade $1 \mathrm{~A}$; ${ }^{\ddagger}, P<0.05$ versus grade $1 \mathrm{~B}$

EMB indicates endomyocardial biopsy; Synd-1, Syndecan-1; MMP-9, matrix metalloproteinase-9

A

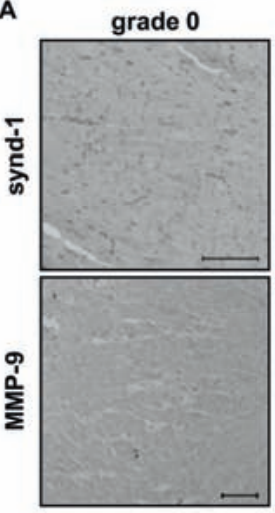

grade $1 \mathrm{~A}$

grade 3

B

synd-1 protein vs $\mathrm{CD}^{+} \mathrm{T}$-cells
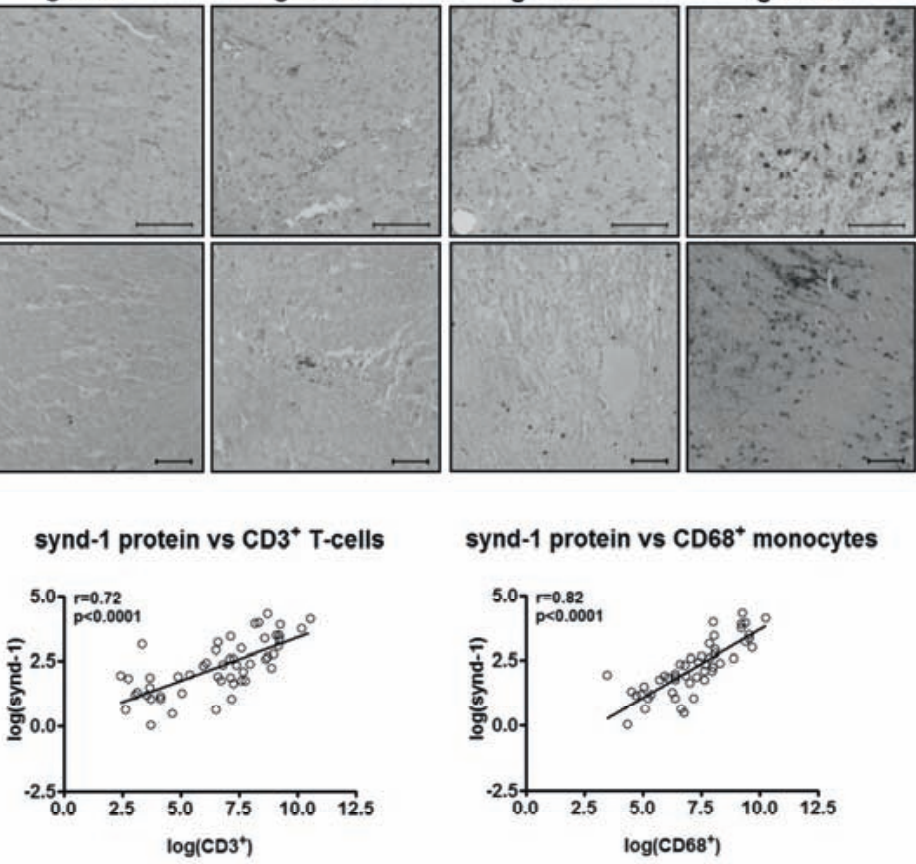

synd-1 protein vs $\mathrm{CD}^{+} 8^{+}$monocytes

C

MMP-9 protein vs $\mathrm{CD}^{+}{ }^{+}$T-cells
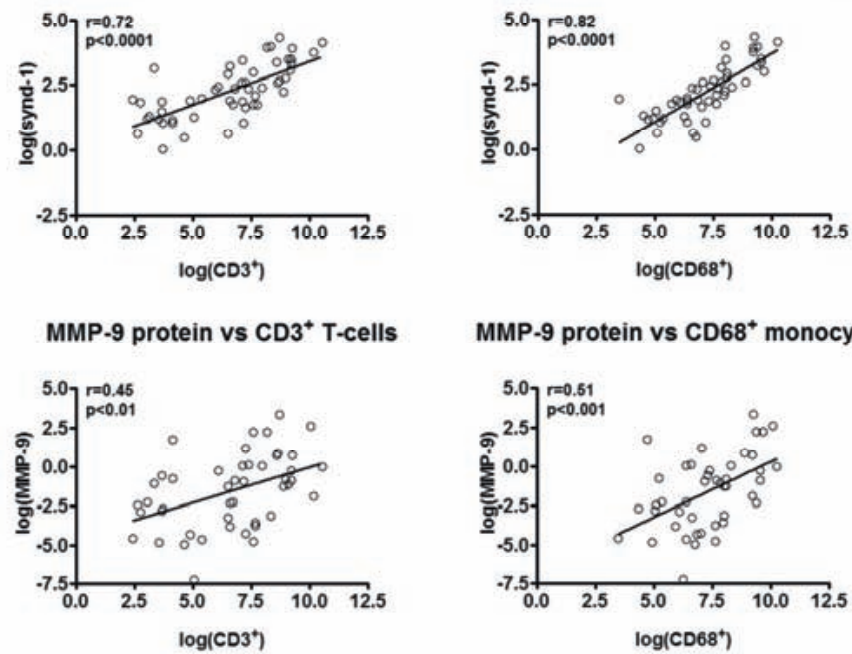

MMP-9 protein vs $\mathrm{CD}^{2} 8^{+}$monocytes

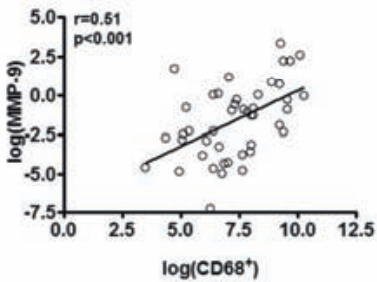

Figure 4. Intragraft Synd-1 and MMP-9 protein expression correlates with the influx of T-cells and monocytes in human CAR. (A) Representative microphotographs Synd-1 and MMP-9 immunoreactivity in EMBs displaying rejection grade $0,1 \mathrm{~A}, 1 \mathrm{~B}$ and 3 . Scale-bar: $100 \mu \mathrm{m}$. (B-C) Immunohistochemical analysis revealed a strong correlation between (B) Synd-1 and (C) MMP-9 and the number of infiltrating CD3 $^{+}$T-cells and $\mathrm{CD}^{+}$monocytes. 


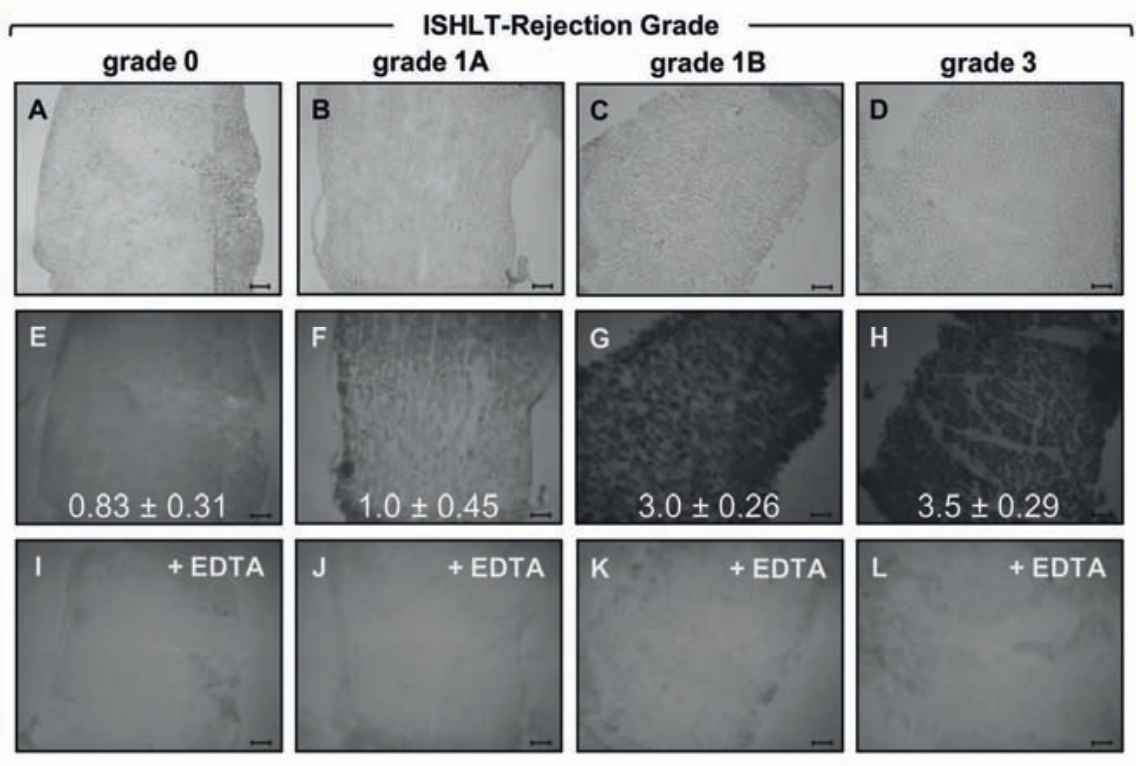

Figure 5. Intragraft In Situ Gelatin Zymography. (A-D) Cryosections were localized using white light microscopy. (E-H) In situ gelatin zymography revealed increasing gelatinolytic (MMP-2 and -9) activity with increasing ISHLT-grade of cardiac allograft rejection $(\mathrm{E}-\mathrm{H}$, increasing gray coloration and indication of average gelatinolytic activity), whereas no activity was seen in parallel sections with inhibition with EDTA (I-L). Scale-bar: $100 \mu \mathrm{m}$.

\section{Discussion}

The present study is the first to investigate the intracardiac transcript, protein and/or activity levels of multiple matricellular proteins, proteoglycans, MMPs and TIMPs in relation to CAR. First, we demonstrate that, out of 14 ECM-related proteins, synd-1 and MMP-9 strongly increase with the degree of CAR, whereas only moderate or no correlations were noted for the other ECM-related proteins included in this study. Intriguingly, our data revealed an even stronger correlation for Synd-1 and MMP-9 with the production of pro-inflammatory cytokines (TNF- $\alpha$, IL- 6 and TGF- $\beta$ ) and with infiltrating $\mathrm{CD}^{+}$T-cells and $\mathrm{CD}^{+} 8^{+}$monocytes. Furthermore, their protein expression robustly co-localized with areas of inflammation and sites of active remodeling. Together, these findings clearly indicate Synd-1 and MMP-9 as potent markers for the inflammatory response in human cardiac allografts during the first critical year after transplantation. On the other hand, our study revealed a strong association for Synd-1, MMP-9, and even more so for TGF- $\beta$ and IL-6, the number of infiltrating T-cells and monocytes, SPARC, CTGF, TSP-1 and -2, TIMP-1 and Testican-1 with collagen transcription in the transplanted heart. However, whereas collagen transcription progressively increased with increasing grade of CAR, analysis of total collagen deposition and MMP2/9 in situ gelatinolytic activity indicated a shift towards higher proteolytic collagen 
degradation in grade 3 rejecting EMBs. All together, these data point towards important molecular interactions between the inflammatory response, non-structural matrix proteins, the metalloproteinase system and cardiac allograft fibrosis in the human transplanted heart.

Interestingly, our findings support a mechanistic implication for Synd-1 and MMP9 in the inflammatory response during CAR, in line with their role as key-regulators of inflammation, extracellular matrix (ECM) remodeling and cellular behavior in response to tissue damage. ${ }^{3,26,27}$ The strong relationship between Synd-1, inflammation and fibrosis in CAR is concordant with its role in a wide variety of inflammatory disorders, such as protein losing enteropathy, ${ }^{28}$ allergic lung inflammation, ${ }^{29}$ acute lung injury, ${ }^{30}$ and exaggerated inflammation in response to myocardial infarction. ${ }^{3}$ Synd- 1 decreases inflammation by binding trough its ectodomain to chemokines, thereby inhibiting chemokine-mediated immune cell migration. ${ }^{31}$ In addition, Synd-1 increases adhesion of inflammatory cells to collagens and limits their invasion into collagen gels. ${ }^{32}$ In line with our findings, Synd-1-positive monocytes were previously associated with rejection of liver and pediatric kidney transplants. ${ }^{13,33}$ Thus, whether upregulation of Synd-1 aims to limit the inflammatory influx, and thereby might protect the transplanted heart should be the subject of further animal studies. In contrast, the involvement of MMP-9 has been addressed in an acute model of CAR in knockout mice, revealing that MMP-9 mediates the influx of mononuclear cells into the allograft, and regulates the activation and expansion of alloreactive T-cells. ${ }^{18}$ Furthermore, Eaton and colleagues ${ }^{19}$ showed that systemic use of a broad-spectrum MMP-inhibitor (GM6001) enhanced allograft survival by directly suppressing the infiltration of inflammatory cells in a mouse model of CAR. In agreement with these reports, our findings are consistent with a key biological role for Synd-1 and MMP-9 in the pathogenesis of CAR In addition, we found that the plasma levels of MMP-9 in our cohort of patients significantly increased with the degree of CAR (Supplemental Table 4). This is in line with previous finding where circulatory levels of MMP-9 related to rejection of liver and kidney transplants. ${ }^{34-36}$. Together, these findings emphasize that the analysis of Synd-1 and MMP-9 in EMBs could withhold a greater sensitivity or predictive value to detect the inflammatory response and related degree of human CAR, as compared the current H\&Ebased ISHLT grading system. ${ }^{1}$ Nevertheless, additional research using larger patient populations will be required to unveil the exact biological and clinical significance of these findings.

Secondly, Our study is - to our knowledge - the first to support the concept that pro-inflammatory cytokines, T-cells and monocytes are closely associated with or even in part responsible for the induction of several non-structural matrix proteins in the human transplanted heart. It is well established that CAR is the result of a highly complex, multiplayer immunologic reaction, in which pro-inflammatory cytokines (such as TNF- $\alpha$, IL- 6 and TGF- $\beta$ ), T-cells and monocytes play a prominent role. ${ }^{1,23,25}$ Our results extend these findings with the observation that TNF- $\alpha$, IL- 6 and TGF- $\beta$, but also the influx of $\mathrm{CD}^{+} \mathrm{T}$-cells and $\mathrm{CD} 8^{+}$monocytes strongly relate to each other and to the 
grade of human CAR. Moreover, the presence of these pro-inflammatory cytokines Tcells and/or monocytes is strongly paralleled by increased expression of primarily Synd-1 and MMP-9, but also CTGF, SPARC, MMP-1, TIMP-1, TSP-1 and -2. These observations are in agreement with previous reports, ranging from the expression of TSP- $1^{8}$, TSP- $2^{10}$, SPARC ${ }^{12}$ and synd $-1^{13}$ by infiltrating inflammatory cells in mouse corneal transplants or human kidney transplants; increased CTGF transcript levels in a mouse model of chronic cardiac allograft rejection ${ }^{11}$; to the immunomodulatory properties of TSP- 1 and $-2^{9}$, Synd $-1^{13}$ and $-4^{14}$ and CTGF ${ }^{11}$ towards T-cells and macrophages. In addition, the majority of these ECM-related matri-cellular proteins modulate the assembly and maturation of the fibrous collagen scar during infarct healing. ${ }^{3,15,16}$ In addition, various animal studies have implied these non-structural matrix proteins, MMPs and TIMPs as important regulators of cardiac disease, inflammation, ECM remodeling and failure. $^{3-7,27}$ Our findings are, therefore, very likely to be indicative of a pivotal role for these proteins in the complex pathways underlying human CAR.

Besides inflammation, cardiac allograft fibrosis has been recently been recognized as a hallmark of CAR. And although its origins are not yet fully understood, fibrosis has been identified as an important limiting factor towards graft function and long-term survival. ${ }^{23}$ We reveal that the production of pro-inflammatory cytokines IL-6 and TGF$\beta$; the influx of T-cells and monocytes; and the expression of Synd-1, TSP-1 and -2 , CTGF, SPARC, Testican-1, MMP-2, MMP-9, TIMP-1 and -2 all strongly relate to the expression of Collagen la1 and Illa1 in the transplanted hearts. Moreover, collagen transcript levels progressively increased with the degree of CAR. Interesting in this regard are the observations that both IL- 6 and TGF- $\beta$ have a negative impact on graft survival through their chemotactic and pro-fibrotic effects. ${ }^{11,23,37,38}$ In addition, IL- $6^{23}$ and TGF$\beta^{23,38}$, but also Synd $-1^{3}$, TSP-1 and $-2^{39}, \mathrm{CTGF}^{23}$, SPARC $^{4,40}$, TIMP-1 and $-2^{17,41}$ were reported to promote fibroblast proliferation or regulate ECM production by promoting collagen synthesis, assembly and/or maturation in the heart. Whereas other suggested that TSP- ${ }^{42}, \mathrm{CTGF}^{11}, \mathrm{MMP}-2^{43}, \mathrm{TIMP}-2^{43}$, and TGF- $\beta^{11,23}$ may directly affect cardiac allograft vasculopathy. The present study translates these findings to the human clinical situation and indicates that Synd-1, TSP-1 and -2, CTGF, SPARC, testican-1, MMP-9, MMP-2, TIMP-1 and -2 might withhold important targets and mechanistic insights to limit the deleterious fibrotic response, cardiac allograft vasculopathy and progressive loss of function of the human transplanted heart. Nevertheless, more in-depth investigations, using a larger cohort of patients, should be conducted to elucidate their exact biological role and predictive value during the onset and progression of CAR.

A final intriguing observation from our study was the fact that the total collagen deposition within our cohort of EMBs did not reflect the increasing transcript levels of COLla1 and COLIIla1 with increasing grade of CAR. This could be the result of increased MMP-9 mediated collagen degradation as suggested by the extensively increased MMP-9 protein levels and MMP in situ proteolytic activity observed in grade 3 rejecting EMBs. ${ }^{15,27}$ Therefore, it might be of future interest to evaluate changes in intragraft and circulating markers of collagen synthesis and degradation in relation to the degree 
of human CAR, including $\mathrm{N}$-terminal propeptides of procollagen I and III (PINP and PIIINP) and carboxyterminal telopeptides (ICTP) respectively. ${ }^{44}$ In this regard, Querejeta R. and colleagues recently showed that PINP, measured in peripheral blood, is a useful marker of myocardial fibrosis and failure in patients with hypertensive heart disease $^{45}$, whereas others have identified that ICTP could withhold a prognostic value following MI. ${ }^{46}$ Nevertheless, additional research will be required to unveil the significance of these markers as diagnostic or prognostic tools for cardiac allograft patients.

In conclusion, we identified Synd-1 and MMP-9 as novel surrogate markers for the degree of inflammation in the transplanted heart, suggesting that their expression may associate with a more integral role in the pathogenesis of CAR. Furthermore, we established a close association between the immunologic response and the induction of non-structural matrix proteins and collagens in the transplanted heart. Therefore, future efforts to elucidate the interplay between these factors may direct the development of improved diagnostic tools and targeted therapies to prevent human CAR.

\section{Acknowledgments}

The authors would like to thank S. Martin from the Leuven Heart Transplant Program and the staff of the Cardiac Catheterization Laboratory at the University Hospital Leuven (K.U.Leuven, Leuven, Belgium) for their assistance in obtaining endomyocardial biopsy specimens and blood samples for this study. We also thank M. Swinnen, S. Jochems and K. Smeekes for their technical assistance. 


\section{References}

1 Stewart S, Winters GL, Fishbein MC, Tazelaar HD, Kobashigawa J, Abrams J, Andersen CB, Angelini A, Berry GJ, Burke MM, Demetris AJ, Hammond E, Itescu S, Marboe CC, McManus B, Reed EF, Reinsmoen NL, Rodriguez ER, Rose AG, Rose M, Suciu-Focia N, Zeevi A, Billingham ME. Revision of the 1990 working formulation for the standardization of nomenclature in the diagnosis of heart rejection. J Heart Lung Transplant. 2005;24(11):1710-1720.

2 Lechler RI, Sykes M, Thomson AW, Turka LA. Organ transplantation--how much of the promise has been realized? Nat Med. 2005;11(6):605-613.

3 Vanhoutte D, Schellings MW, Gotte M, Swinnen M, Herias V, Wild MK, Vestweber D, Chorianopoulos E, Cortes V, Rigotti A, Stepp MA, Van de Werf F, Carmeliet P, Pinto YM, Heymans S. Increased expression of syndecan-1 protects against cardiac dilatation and dysfunction after myocardial infarction. Circulation. 2007;115(4):475-482.

4 Schellings MW, Vanhoutte D, Swinnen M, Cleutjens JP, Debets J, van Leeuwen RE, d'Hooge J, Van de Werf F, Carmeliet P, Pinto YM, Sage EH, Heymans S. Absence of SPARC results in increased cardiac rupture and dysfunction after acute myocardial infarction. J Exp Med. 2009;206(1):113-123.

5 Schroen B, Heymans S, Sharma U, Blankesteijn WM, Pokharel S, Cleutjens JP, Porter JG, Evelo CT, Duisters R, van Leeuwen RE, Janssen BJ, Debets JJ, Smits JF, Daemen MJ, Crijns HJ, Bornstein P, Pinto YM. Thrombospondin-2 is essential for myocardial matrix integrity: increased expression identifies failureprone cardiac hypertrophy. Circ Res. 2004;95(5):515-522.

6 Swinnen M, Vanhoutte D, Van Almen GC, Hamdani N, Schellings MW, D'Hooge J, Van der Velden J, Weaver MS, Sage EH, Bornstein P, Verheyen FK, VandenDriessche T, Chuah MK, Westermann D, Paulus WJ, Van de Werf F, Schroen B, Carmeliet P, Pinto YM, Heymans S. Absence of thrombospondin-2 causes age-related dilated cardiomyopathy. Circulation. 2009;120(16):1585-1597.

7 Westermann D, Savvatis K, Schultheiss HP, Tschope C. Immunomodulation and matrix metalloproteinases in viral myocarditis. J Mol Cell Cardiol. 2010;48(3):468-473.

8 Saban DR, Bock F, Chauhan SK, Masli S, Dana R. Thrombospondin-1 derived from APCs regulates their capacity for allosensitization. J Immunol. 2010;185(8):4691-4697.

9 Lamy L, Foussat A, Brown EJ, Bornstein P, Ticchioni M, Bernard A. Interactions between CD47 and thrombospondin reduce inflammation. J Immunol. 2007;178(9):5930-5939.

10 Rodder S, Scherer A, Raulf F, Berthier CC, Hertig A, Couzi L, Durrbach A, Rondeau E, Marti HP. Renal allografts with IF/TA display distinct expression profiles of metzincins and related genes. Am J Transplant. 2009;9(3):517-526.

11 Booth AJ, Csencsits-Smith K, Wood SC, Lu G, Lipson KE, Bishop DK. Connective tissue growth factor promotes fibrosis downstream of TGFbeta and IL-6 in chronic cardiac allograft rejection. Am J Transplant. 2010;10(2):220-230.

12 Alpers CE, Hudkins KL, Segerer S, Sage EH, Pichler R, Couser WG, Johnson RJ, Bassuk JA. Localization of SPARC in developing, mature, and chronically injured human allograft kidneys. Kidney Int. 2002;62(6):2073-2086.

13 Tsai EW, Wallace WD, Gjertson DW, Reed EF, Ettenger RB. Significance of intragraft CD138+ lymphocytes and p-S6RP in pediatric kidney transplant biopsies. Transplantation. 2010;90(8):875-881.

14 Li J, Brown LF, Laham RJ, Volk R, Simons M. Macrophage-dependent regulation of syndecan gene expression. Circ Res. 1997;81(5):785-796.

15 Dobaczewski M, Gonzalez-Quesada C, Frangogiannis NG. The extracellular matrix as a modulator of the inflammatory and reparative response following myocardial infarction. J Mol Cell Cardiol. 2010;48(3):504-511.

16 Schellings MW, Pinto YM, Heymans S. Matricellular proteins in the heart: possible role during stress and remodeling. Cardiovasc Res. 2004;64(1):24-31.

17 Vanhoutte D, Heymans S. TIMPs and cardiac remodeling: 'Embracing the MMP-independent-side of the family'. J Mol Cell Cardiol. 2010;48(3):445-453. 
18 Campbell LG, Ramachandran S, Liu W, Shipley JM, Itohara S, Rogers JG, Moazami N, Senior RM, Jaramillo A. Different roles for matrix metalloproteinase-2 and matrix metalloproteinase- 9 in the pathogenesis of cardiac allograft rejection. Am J Transplant. 2005;5(3):517-528.

19 Eaton VL, Lerret NM, Velasquez-Lopera MM, John R, Caicedo M, DeCresce RP, Jaramillo A. Enhanced allograft survival and modulation of T-cell alloreactivity induced by inhibition of MMP/ADAM enzymatic activity. Am J Transplant. 2008;8(3):507-516.

20 Ogawa M, Suzuki J, Takayama K, Isobe M. Matrix metalloproteinase suppression induced by clarithromycin in murine cardiac allografts. Transplant Proc. 2009;41(1):395-397.

21 Aharinejad S, Krenn K, Zuckermann A, Schafer R, Paulus P, Seebacher G, Wolner E, Grimm M. Matrix metalloproteases and their tissue inhibitor in cardiac transplantation. Eur J Cardiothorac Surg. 2007;32(1):48-51.

22 Schupp DJ, Huck BP, Sykora J, Flechtenmacher C, Gorenflo M, Koch A, Sack FU, Haass M, Katus HA, Ulmer HE, Hagl S, Otto HF, Schnabel PA. Right ventricular expression of extracellular matrix proteins, matrix-metalloproteinases, and their inhibitors over a period of 3 years after heart transplantation. Virchows Arch. 2006;448(2):184-194.

23 Booth AJ, Bishop DK. TGF-beta, IL-6, IL-17 and CTGF direct multiple pathologies of chronic cardiac allograft rejection. Immunotherapy. 2010;2(4):511-520.

24 de Groot-Kruseman HA, Mol WM, Niesters HG, Maat AP, van Gelder T, Balk AH, Weimar W, Baan CC. Differential intragraft cytokine messenger RNA profiles during rejection and repair of clinical heart transplants. A longitudinal study. Transpl Int. 2003;16(1):9-14.

25 Azzawi M, Hasleton P. Tumour necrosis factor alpha and the cardiovascular system: its role in cardiac allograft rejection and heart disease. Cardiovasc Res. 1999;43(4):850-859.

26 Spinale FG. Myocardial matrix remodeling and the matrix metalloproteinases: influence on cardiac form and function. Physiol Rev. 2007;87(4):1285-1342.

27 Vanhoutte D, Schellings M, Pinto Y, Heymans S. Relevance of matrix metalloproteinases and their inhibitors after myocardial infarction: a temporal and spatial window. Cardiovasc Res. 2006;69(3):604-613.

28 Bode L, Salvestrini C, Park PW, Li JP, Esko JD, Yamaguchi Y, Murch S, Freeze HH. Heparan sulfate and syndecan-1 are essential in maintaining murine and human intestinal epithelial barrier function. J Clin Invest. 2008;118(1):229-238.

29 Xu J, Park PW, Kheradmand F, Corry DB. Endogenous attenuation of allergic lung inflammation by syndecan-1. J Immunol. 2005;174(9):5758-5765.

30 Li Q, Park PW, Wilson CL, Parks WC. Matrilysin shedding of syndecan-1 regulates chemokine mobilization and transepithelial efflux of neutrophils in acute lung injury. Cell. 2002;111(5):635-646.

31 Bartlett AH, Hayashida K, Park PW. Molecular and cellular mechanisms of syndecans in tissue injury and inflammation. Mol Cells. 2007;24(2):153-166.

32 Liu W, Litwack ED, Stanley MJ, Langford JK, Lander AD, Sanderson RD. Heparan sulfate proteoglycans as adhesive and anti-invasive molecules. Syndecans and glypican have distinct functions. J Biol Chem. 1998;273(35):22825-22832.

33 Krukemeyer MG, Moeller J, Morawietz L, Rudolph B, Neumann U, Theruvath T, Neuhaus P, Krenn V. Description of $B$ lymphocytes and plasma cells, complement, and chemokines/receptors in acute liver allograft rejection. Transplantation. 2004;78(1):65-70.

34 Aharinejad S, Krenn K, Zuckermann A, Schafer R, Gmeiner M, Thomas A, Aliabadi A, Schneider B, Grimm M. Serum matrix metalloprotease-1 and vascular endothelial growth factor--a predict cardiac allograft rejection. Am J Transplant. 2009;9(1):149-159.

35 Kuyvenhoven JP, Molenaar IQ, Verspaget HW, Veldman MG, Palareti G, Legnani C, Moolenburgh SE, Terpstra OT, Lamers CB, van Hoek B, Porte RJ. Plasma MMP-2 and MMP-9 and their inhibitors TIMP-1 and TIMP-2 during human orthotopic liver transplantation. The effect of aprotinin and the relation to ischemia/reperfusion injury. Thromb Haemost. 2004;91(3):506-513.

36 Rodrigo E, Lopez Hoyos M, Escallada R, Ruiz JC, Fresnedo GF, Heras M, Pinera C, Ramos MA, Cotorruelo JG, Arias M. Changes in serum concentrations of matrix metalloproteinases in kidney transplantation. Transplant Proc. 2000;32(3):517-518. 
37 Aziz TM, Burgess MI, Haselton PS, Yonan NA, Hutchinson IV. Transforming growth factor beta and diastolic left ventricular dysfunction after heart transplantation: echocardiographic and histologic evidence. J Heart Lung Transplant. 2003;22(6):663-673.

38 Bujak M, Frangogiannis NG. The role of TGF-beta signaling in myocardial infarction and cardiac remodeling. Cardiovasc Res. 2007;74(2):184-195.

39 Schellings MW, van Almen GC, Sage EH, Heymans S. Thrombospondins in the heart: potential functions in cardiac remodeling. J Cell Commun Signal. 2009;3(3-4):201-213.

40 McCurdy S, Baicu CF, Heymans S, Bradshaw AD. Cardiac extracellular matrix remodeling: Fibrillar collagens and Secreted Protein Acidic and Rich in Cysteine (SPARC). J Mol Cell Cardiol. 2009;48(3):544-549.

41 Heymans S, Schroen B, Vermeersch P, Milting H, Gao F, Kassner A, Gillijns H, Herijgers P, Flameng W, Carmeliet $P$, Van de Werf F, Pinto YM, Janssens S. Increased cardiac expression of tissue inhibitor of metalloproteinase-1 and tissue inhibitor of metalloproteinase- 2 is related to cardiac fibrosis and dysfunction in the chronic pressure-overloaded human heart. Circulation. 2005;112(8):1136-1144.

42 Zhao XM, Hu Y, Miller GG, Mitchell RN, Libby P. Association of thrombospondin-1 and cardiac allograft vasculopathy in human cardiac allografts. Circulation. 2001;103(4):525-531.

43 Tsukioka K, Suzuki J, Fujimori M, Wada Y, Yamaura K, Ito K, Morishita R, Kaneda Y, Isobe M, Amano J. Expression of matrix metalloproteinases in cardiac allograft vasculopathy and its attenuation by anti MMP-2 ribozyme gene transfection. Cardiovasc Res. 2002;56(3):472-478.

44 Lopez B, Gonzalez A, Diez J. Circulating biomarkers of collagen metabolism in cardiac diseases. Circulation. 2010;121(14):1645-1654.

45 Querejeta R, Lopez B, Gonzalez A, Sanchez E, Larman M, Martinez Ubago JL, Diez J. Increased collagen type I synthesis in patients with heart failure of hypertensive origin: relation to myocardial fibrosis. Circulation. 2004;110(10):1263-1268.

46 Manhenke C, Orn S, Squire I, Radauceanu A, Alla F, Zannad F, Dickstein K. The prognostic value of circulating markers of collagen turnover after acute myocardial infarction. Int J Cardiol. 2010;doi:10.1016/j.ijcard.2010.04.034.

47 Heymans S, Lupu F, Terclavers S, Vanwetswinkel B, Herbert JM, Baker A, Collen D, Carmeliet P, Moons L. Loss or inhibition of UPA or MMP-9 attenuates LV remodeling and dysfunction after acute pressure overload in mice. Am J Pathol. 2005;166(1):15-25.

48 Lehoux S, Lemarie CA, Esposito B, Lijnen HR, Tedgui A. Pressure-induced matrix metalloproteinase-9 contributes to early hypertensive remodeling. Circulation. 2004;109(8):1041-1047. 


\section{Chapter 6}

\section{Supplemental material}

\section{Expanded methods}

RNA isolation and real-time polymerase chain reaction

RNA was isolated from EMBs displaying ISHLT grade $0(n=35)$, grade 1 A $(n=29)$, grade $1 B(n=27)$ and grade $3(n=8)$, using the RNeasy Fibrous tissue Mini kit (Qiagen $\mathrm{GmbH}){ }^{1}$, ${ }^{2}$ RNA quality and concentration was determined by spectrophotometry using the Ultrospec 2100 pro UV/Visible spectrophotometer (Amersham Biosciences). One hundred nanogram of total RNA was reverse-transscribed into cDNA using the Qiagan Quantitect Reverse trancription kit (Qiagen $\mathrm{GmbH}$ ). Next, one nanogram of cDNA was amplified and detected with the Applied Biosystems 7500 Fast Real-Time PCR System (PE Applied Biosystems) to determine transcript levels of connective tissue growth factor (CTGF), secreted protein acidic and rich in cysteine (SPARC), syndecan-1, syndecan-4, testican, thrombospondin-1 (TSP-1), thrombospondin-2 (TSP-2), MMP-1, MMP2, MMP-9, TIMP-1, TIMP-2, TIMP-3 and TIMP-4. ${ }^{1}$ In order to relate the degree of CAR with the extent of inflammatory markers and the extent of fibrosis present in the biopsy specimen, cardiac expression of interleukin- 6 (IL-6), tumor necrosis factor- $\alpha$ (TNF$\alpha$ ), transforming growth factor- $\beta 1$ (TGF- $\beta 1$ ), and collagen type I and type III was measured. Primer and probe sequences are listed in supplemental table 2. Transcript levels were determined in duplicate, and expressed as arbitrary units, relative to GAPDH expression.

\section{Histological analysis}

After routine histology to determine the ISHLT-rejection grade, the bouin-fixed and paraffin embedded EMBs were retrospectively collected and sectioned at $6 \mu \mathrm{m}$. An average of at least 3 biopsies per patient and per catheterization were analyzed in this study. To increase the number of ISHLT-grade 3 rejecting EMBs in our histological analysis, additional grade 3 rejecting EMBs were collected from 7 patients that received a heart transplant in our center between 1999 and 2005. Primary antibodies against CD3 (A0452), CD68 (M0814), syndecan-1 (M7228; all DAKO, Carpenteria, CA, USA), MMP1 (AF901), MMP2 (AF902), MMP9 (AF9011), TIMP1 (AF970), TIMP2 (AF971), and TIMP3 (MAB973) (all R\&D systems, Wiesbaden-Nordenstadt, Germany) were used for immunostaining, according to standard protocol and as previously described. ${ }^{1}$ Shortly, twenty minutes of antigen retrieval in DAKO Target Retrieval Solution (Dako, Carpinteria, USA) at $90^{\circ} \mathrm{C}$ was performed on cross-sections of the EMBs. Next, endogenous 
peroxidase activity was blocked by incubating the slides for 20 minute in methanol containing $0.3 \% \mathrm{H} 202$. Prior to staining, aspecific binding sites were masked by incubation with appropriate serum (rabbit or goat serum). Sections were stained by overnight incubation with primary antibodies diluted in $100 \mathrm{mM}$ Tris. $\mathrm{HCl}, \mathrm{Ph} 7.6$, containing $0.9 \% \mathrm{NaCl}, 0.1 \%$ triton $\mathrm{X}-100$ and $2 \%$ bovine serum albumine. Peroxidase (PO) labeled secondary antibodies were used and peroxidase activity was visualized using $0.06 \%$ $3,3^{\prime}$-diaminobenzidine (DAB)/ $0.01 \% \mathrm{H}_{2} \mathrm{O}_{2}$ in $0.05 \mathrm{M}$ Tris. $\mathrm{HCl}, \mathrm{pH}$ 7.0. If necessary, the signal was enhanced by using TSA kit (Perkin Elmer, Boston, USA) based on straptavidin-PO and Biotinyl tyramide amplification. Finally, sections were counterstained with Harris' hematoxylin, dehydrated and mounted in DPX (Prosan, Ghent, Belgium). For each primary antibody used, staining specificity was confirmed on a control section by applying the same protocol with omission of the primary antibody or by using irrelevant isotype control antibodies. Syndecan-1, MMP and TIMP immunoreactivity was expressed as percentage staining area per total tissue area. In order to measure the number of leukocytes and monocytes in the biopsy specimen, CD3 and CD68 positive cells were counted, respectively, and expressed per square millimeter. The collagen content in the endomyocardial regions was determined by picrosirius-red staining and presented as percentage from the total tissue area. Morphometrical analyses were performed on three sections per EMB, according to standard operating procedures and confirmed by independent observers blinded to the to the degree of CAR. Microscopic images were taken and analyzed using a Zeiss Axioplan2 microscope, a 3CCD video camera (DXC-930P, Sony) and KS300 software as previously described. ${ }^{1,3}$

\section{In situ gelatin zymography}

MMP2/9-in situ gelatin zymography was performed as previously described. ${ }^{1,4}$ Briefly, $8 \mu \mathrm{m}$ thick cryosections of rejecting and non-rejecting biopsies (arbitrarily chosen, $\mathrm{n}=21$ ) were covered with fluorescently labeled pigskin gelatin (Oregon Green 488, Molecular probes) and incubated for 48 hours at $37^{\circ} \mathrm{C}$. The gelatinase activity, indicated by gray discoloration caused by gelatin degradation, was scored on a scale of $0-$ 4: 0 (no activity); 1 , focal activity; 2 , moderate activity; 3 , diffuse activity and 4, very high activity. To confirm specificity of gelatinase activity in the degradation assay, consecutive sections were coincubated with EDTA, an inhibitor of MMP activity.

\section{Enzyme Linked Immunosorbent assay (ELISA)}

ELISA was performed to detect human pro-MMP-1, MMP-2, MMP-9, TIMP-1 and TIMP2 in consecutive plasma samples obtained from our cohort of $39 \mathrm{HTx}$ patients $(n=230$; see 'tissue sampling'), using Quantikine kits (R\&D Systems) and following manufacturers instruction. 


\section{Supplemental tables}

Supplemental table 1. Patient characteristics

\begin{tabular}{llll}
\hline Gender & Number & Age: Y, median (range) & Age: mean \pm SEM \\
\hline Male & $30(76 \%)$ & $52(7-66)$ & $48 \pm 3$ \\
Female & $9(24 \%)$ & $33(15-64)$ & $37 \pm 6$ \\
Total & $39(100 \%)$ & $51(7-66)$ & $46 \pm 3$ \\
\hline
\end{tabular}

Supplemental table 2. Real-Time PCR primer and probe sequences

\begin{tabular}{|c|c|c|}
\hline Gene & Primer & Sequence $5^{\prime}-3^{\prime}$ \\
\hline \multicolumn{3}{|c|}{ Matrix glycoproteins } \\
\hline CTGF & $\mathrm{F}$ & cacagagtggagcgcctgttc \\
\hline (NM_001901) & $\mathrm{R}$ & gatgcactttttgcccttcttaatg \\
\hline SPARC & $\mathrm{F}$ & tcttccctgtacactggcagttc \\
\hline (NM_003118) & $\mathrm{R}$ & aggtcacaggtctcgaaaaagc \\
\hline syndecan-1 & $\mathrm{F}$ & cttcacactccccacacaga \\
\hline (NM_002997) & $\mathrm{R}$ & ggccactacagccgtattct \\
\hline syndecan-4 & $\mathrm{F}$ & agcactgtgcagggcagcaa \\
\hline (NM_002999) & $\mathrm{R}$ & aggatgcccacgatgccacc \\
\hline Testican-1 & $\mathrm{F}$ & gtgctggtgtgtggacaaat \\
\hline (NM_004598) & $\mathrm{R}$ & gctcccgttcatattctaggtc \\
\hline TSP-1 & $\mathrm{F}$ & ggcaaggactgcgttggt \\
\hline (NM003246) & $\mathrm{R}$ & cacttcacgccggcaaag \\
\hline TSP-2 & $\mathrm{F}$ & tggaaggactacacggcctatag \\
\hline (NM_003247) & $\mathrm{R}$ & taggtttggtcatagataggtcctgagt \\
\hline \multicolumn{3}{|c|}{ MMPs and TIMPS } \\
\hline MMP-1 & $\mathrm{F}$ & cacaactgccaaatgggctt \\
\hline \multirow[t]{2}{*}{ (NM_002421) } & Probe & tctctgtcggcaaattcgtaagcagctt \\
\hline & $\mathrm{R}$ & ccctttgaaaaaccggacttc \\
\hline MMP-2 & $\mathrm{F}$ & atttgatggcatcgctcagat \\
\hline \multirow[t]{2}{*}{ (NM_004530) } & Probe & cttcaaggaccggttcatttggcg \\
\hline & $\mathrm{R}$ & tgtcacgtggcgtcacgt \\
\hline MMP-9 & $\mathrm{F}$ & tggatccaaaactcctcggaaga \\
\hline \multirow[t]{2}{*}{ (NM_004994) } & Probe & cttcactcgcgtgtacagccggga \\
\hline & $\mathrm{R}$ & acaccaaactggatgacgatgtc \\
\hline TIMP-1 & $\mathrm{F}$ & ctgcggatacttccacaggtc \\
\hline \multirow[t]{2}{*}{ (NM_003254) } & Probe & cacaaccgcagcgaggagtttctcat \\
\hline & $\mathrm{R}$ & tccagggagccacgaaact \\
\hline TIMP-2 & $\mathrm{F}$ & tctggatggactgggtcaca \\
\hline \multirow[t]{2}{*}{ (NM_003255) } & Probe & agaagaacatcaacgggcaccaggc \\
\hline & $\mathrm{R}$ & cttgatgcaggcgaagaactt \\
\hline TIMP-3 & $\mathrm{F}$ & gtctacaccatcaagcagatgaag \\
\hline \multirow[t]{2}{*}{ (NM_000362) } & Probe & aggcttcaccaagatgccccatgtg \\
\hline & $\mathrm{R}$ & cagcttaaggccacagagactct \\
\hline TIMP-4 & $\mathrm{F}$ & cgcaccctcagcagcac \\
\hline \multirow[t]{2}{*}{ (NM_003256) } & Probe & ctgccactcggcacttgattcg \\
\hline & $\mathrm{R}$ & ccttctcactggagattttggc \\
\hline
\end{tabular}




\begin{tabular}{lll}
\hline Gene & Primer & Sequence $5^{\prime}-3^{\prime}$ \\
\hline Cytokines & & \\
IL-6 & $\mathrm{F}$ & \\
(NM_000600) & $\mathrm{R}$ & acctgaaccttccaaagatggctga \\
TNF- $\alpha$ & $\mathrm{F}$ & gaccagtgatgattttcaccaggca \\
(NM_000594) & $\mathrm{R}$ & ccctctggcccaggcagtca \\
TGF-B & $\mathrm{F}$ & cggcggttcagccactggag \\
(NM_000660) & $\mathrm{R}$ & ctcgggccctgccctacat \\
& & gcgcccgggttatgctggtt \\
Fibrosis & & \\
collagen 1A1 & $\mathrm{F}$ & \\
(NM_000088) & $\mathrm{R}$ & aggacaagaggcatgtctggtt \\
collagen 3A1 & $\mathrm{F}$ & ggacatcaggcgcaggaa \\
(NM_000090) & $\mathrm{R}$ & tggaggatggttgcacgaa \\
Housekeeping gene & & ccaacgtccacaccaaattctt \\
GAPDH & & \\
(NM_002046) & $\mathrm{F}$ & \\
\hline
\end{tabular}

Primers and probes were designed from the mRNA sequences as annotated in the NCBI GenBank (accessionnumber given in parenthesis). RT-PCR, Real-Time Polymerase Chain Reaction; F, forward; R, reverse; $P$, probe.

Supplemental table 3. MMP/TIMP immunohistochemistry in relation to the different ISHLT-grades of cardiac allograft rejection

\begin{tabular}{lcccccc}
\hline & $\begin{array}{c}\text { Grade 0 } \\
(\mathrm{n}=21)\end{array}$ & $\begin{array}{c}\text { Grade 1A } \\
(\mathrm{n}=19)\end{array}$ & $\begin{array}{c}\text { Grade 1B } \\
(\mathrm{n}=16)\end{array}$ & $\begin{array}{c}\text { Grade 3 } \\
(\mathrm{n}=15)\end{array}$ & \multicolumn{2}{c}{ ANOVA post test for linearity } \\
\cline { 7 - 8 } & & & & & $R^{2}$-value & $P$-value \\
\hline MMP-1, \% & $1.09 \pm 0.16$ & $1.34 \pm 0.18$ & $1.52 \pm 0.22$ & $1.67 \pm 0.24^{*}$ & $\mathrm{R}^{2}=0.046$ & $\mathrm{P}<0.05$ \\
MMP-2, \% & $3.81 \pm 0.84$ & $5.39 \pm 1.18$ & $5.52 \pm 1.46$ & $6.10 \pm 1.35$ & $\mathrm{R}^{2}=0.027$ & n.s. \\
TIMP-1, \% & $22.10 \pm 2.65$ & $23.93 \pm 3.19$ & $25.04 \pm 3.30$ & $29.38 \pm 2.78^{*}$ & $\mathrm{R}^{2}=0.036$ & n.s. \\
TIMP-2, \% & $8.17 \pm 0.90$ & $7.52 \pm 0.81$ & $9.56 \pm 0.78$ & $8.51 \pm 1.00$ & $\mathrm{R}^{2}=0.011$ & n.s. \\
TIMP-3, \% & $0.83 \pm 0.21$ & $1.65 \pm 0.36$ & $1.74 \pm 0.42$ & $3.97 \pm 0.82^{*+\neq}$ & $\mathrm{R}^{2}=0.231$ & $P<0.0001$ \\
\hline
\end{tabular}

ISHLT-grade indicates the histopathological grade of cardiac allograft rejection as set by the International Society of Heart and Lung Transplantation. Protein levels are given as percentage positive staining area relative to total tissue area and presented as mean $\pm \mathrm{SEM} .{ }^{*}, P<0.05$ versus grade $0 ;{ }^{\dagger}, P<0.05$ versus grade $1 \mathrm{~A} ;{ }^{\ddagger}, P<0.05$ versus grade $1 \mathrm{~B}$

MMP stands for matrix metalloproteinase; TIMP, tissue inhibitor of matrix metalloproteinases; n.s., not significant. 
Supplemental table 4. MMP/TIMP plasma levels in relation to the different ISHLT-grades of cardiac allograft rejection

\begin{tabular}{|c|c|c|c|c|c|c|}
\hline & \multirow{2}{*}{$\begin{array}{l}\text { Grade } 0 \\
(n=117)\end{array}$} & \multirow{2}{*}{$\begin{array}{c}\text { Grade } 1 A \\
(n=60)\end{array}$} & \multirow{2}{*}{$\begin{array}{l}\text { Grade 1B } \\
(n=36)\end{array}$} & \multirow{2}{*}{$\begin{array}{c}\text { Grade } 3 \\
(n=8)\end{array}$} & \multicolumn{2}{|c|}{ ANOVA post test for linearity } \\
\hline & & & & & $R^{2}$-value & P-value \\
\hline Pro-MMP1 & $2.29 \pm 0.13$ & $2.16 \pm 0.20$ & $1.99 \pm 0.22$ & $2.82 \pm 0.75$ & $R^{2}=0.004$ & n.s. \\
\hline MMP2 & $26.32 \pm 1.24$ & $26.29 \pm 1.02$ & $25.15 \pm 1.15$ & $26.42 \pm 1.22$ & $\mathrm{R}^{2}<0.001$ & n.s. \\
\hline MMP9 & $1.47 \pm 0.08$ & $1.72 \pm 0.17$ & $2.43 \pm 0.41^{*}$ & $2.79 \pm 0.83^{*}$ & $R^{2}=0.041$ & $P<0.01$ \\
\hline TIMP1 & $1.86 \pm 0.12$ & $1.71 \pm 0.17$ & $1.83 \pm 0.07$ & $1.89 \pm 0.18$ & $R^{2}<0.001$ & n.s. \\
\hline TIMP2 & $1.50 \pm 0.09$ & $1.36 \pm 0.08$ & $1.39 \pm 0.10$ & $1.25 \pm 0.15$ & $R^{2}=0.015$ & n.s. \\
\hline
\end{tabular}

ISHLT-grade indicates the histopathological grade of cardiac allograft rejection as set by the International Society of Heart and Lung Transplantation. Plasma levels of MMPs and TIMPs are given in nanograms protein per milliliter plasma and presented as mean \pm SEM. ${ }^{*}, P<0.05$ vs grade 0 .

MMP stands for matrix metalloproteinase; TIMP, tissue inhibitor of matrix metalloproteinases; and n.s., not significant. 


\section{Supplemental figures}

A

syndecan- 4 vs IL- 6

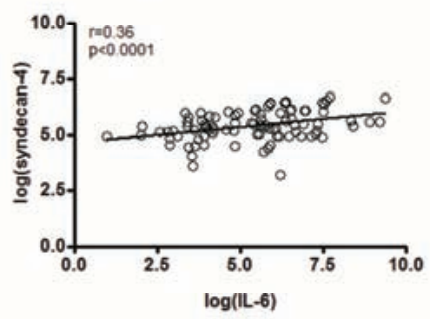

B

TSP-1 vs IL-6

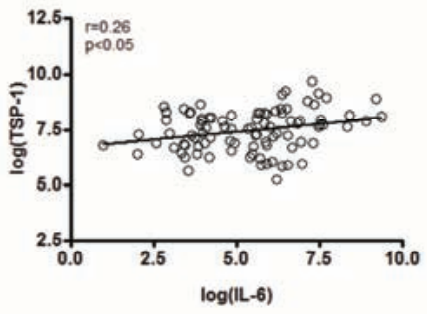

C

TSP-2 vs IL-6

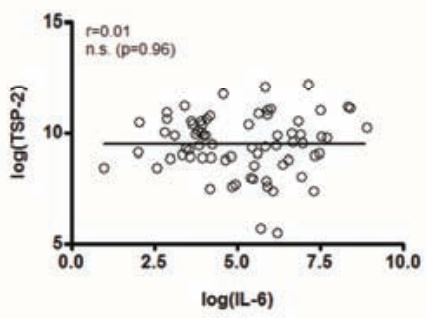

D

testican vs IL-6

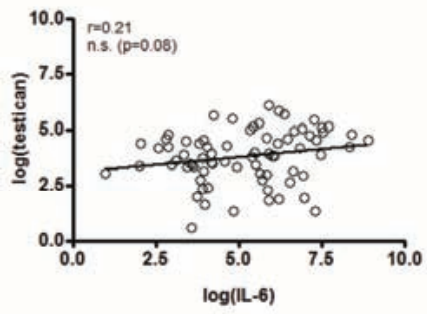

syndecan- 4 vs TNF- $\alpha$

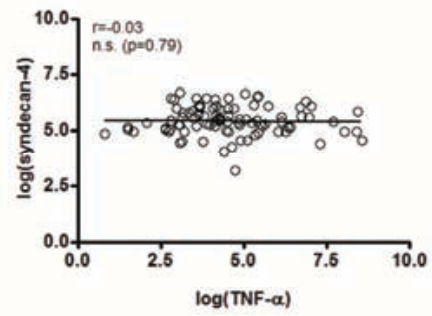

TSP-1 vS TNF- $\alpha$

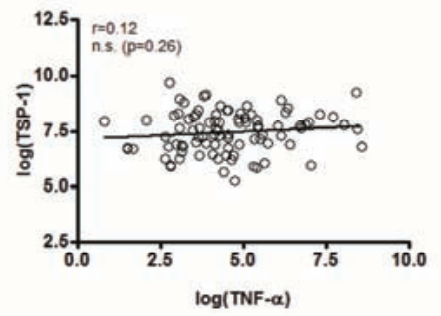

TSP-2 VS TNF- $\alpha$

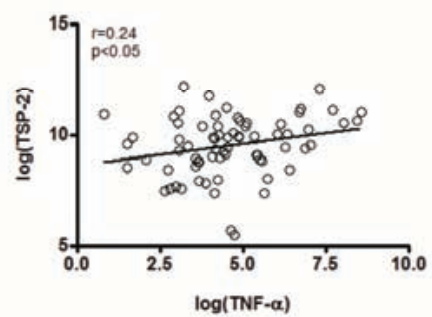

testican vs TNF- $\alpha$

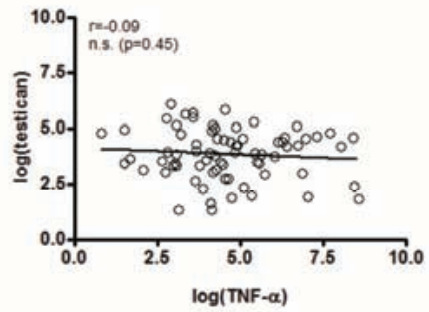

syndecan- 4 vs TGF- $\beta$

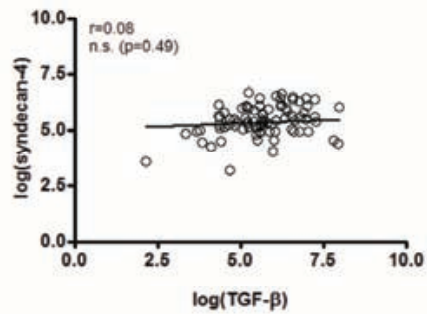

TSP-1 vs TGF- $\beta$

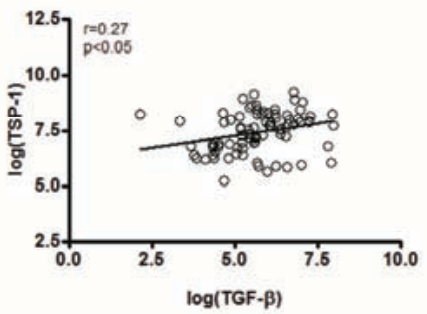

TSP-2 vS TGF- $\beta$

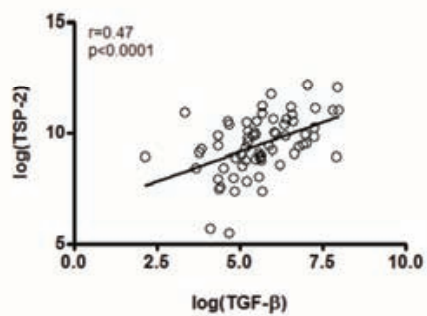

testican vs TGF- $\beta$

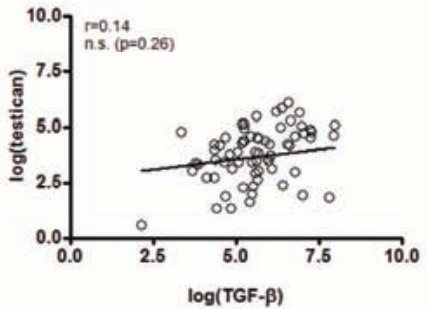

Supplemental figure 1A-D. Intragraft transcript levels in relation to the expression of pro-inflammatory cytokines. Supplemental figure $1 \mathrm{E}-\mathrm{H}$ continued on page 160 
E

MMP-2 vs IL-6

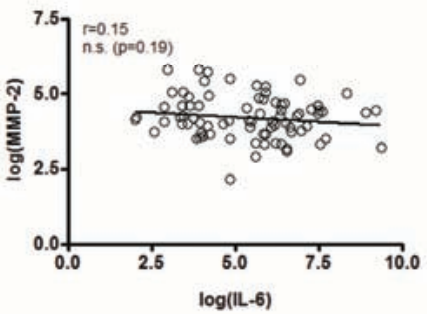

$\mathbf{F}$

TIMP-2 vs IL-6

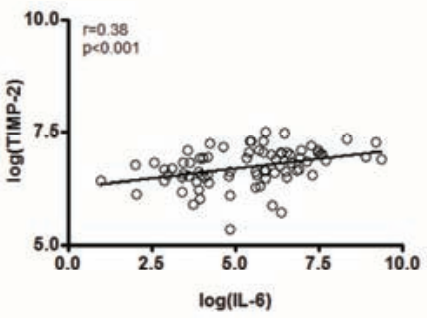

$\mathbf{G}$

TIMP-3 vs IL-6

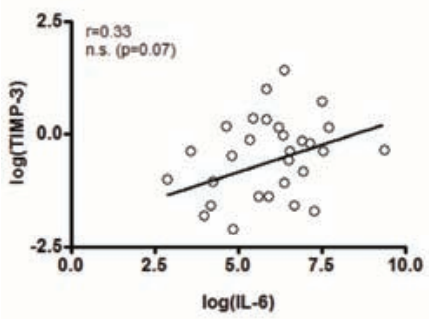

H

TIMP-4 VS IL-6

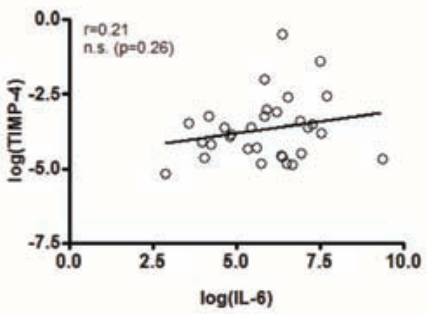

MMP-2 vs TNF- $\alpha$

MMP-2 vs TGF- $\beta$
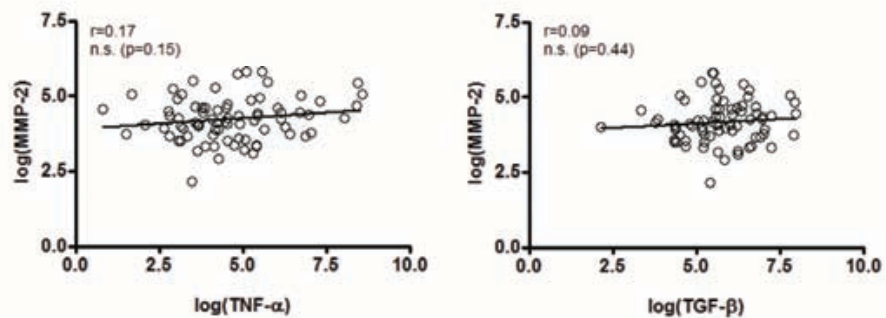

TIMP-2 vs TGF- $\beta$

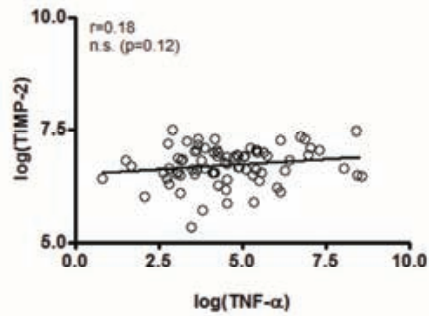

TIMP-3 vs TNF- $\alpha$

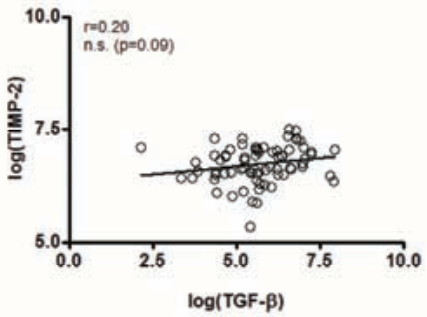

TIMP-3 vs TGF- $\beta$

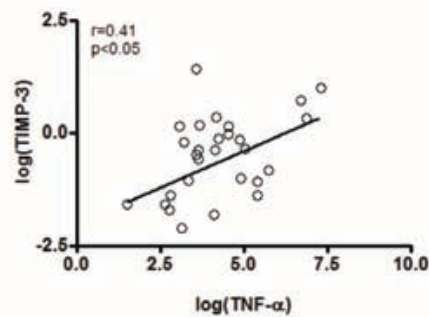

TIMP- 4 VS TNF- $\alpha$

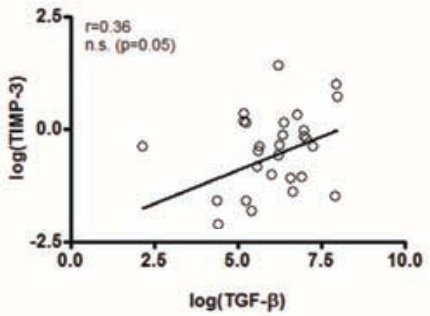

TIMP -4 vs TGF- $\beta$

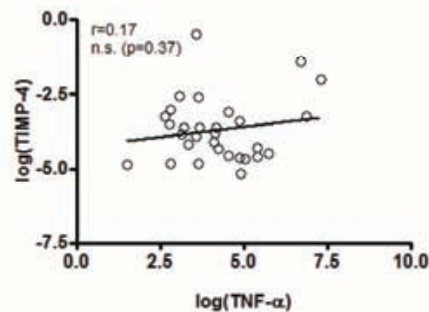

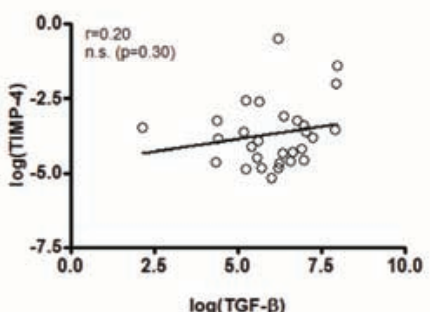

Supplemental figure 1. Intragraft transcript levels in relation to the expression of pro-inflammatory cytokines. Intragraft transcript levels of (A) Syndecan-4, (B) TSP-1, (C) TSP-2, (D) Testican-1, (E) MMP-1, (F) TIMP2, (G) TIMP-3 and (H) TIMP-4 in relation to the mRNA expression levels of IL-6, TNF- $\alpha$, and TGF- $\beta$ ( $n=102$, including $n=38$ for ISHLT grade $0, n=31$ for grade $1 A, n=25$ for grade $1 B$ and $n=8$ for grade 3 ). 
A

syndecan-4 vs CD3

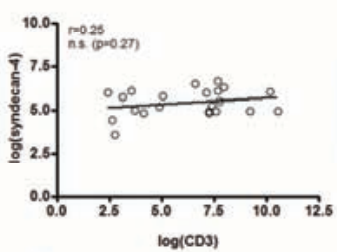

C

MMP-1 vs CD3

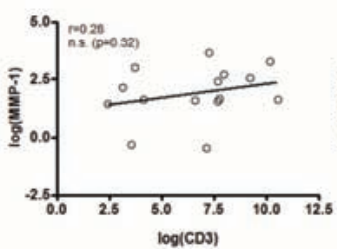

E

TIMP-2 vs CD3

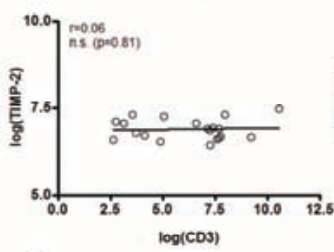

G syndecan-4 vs CD68

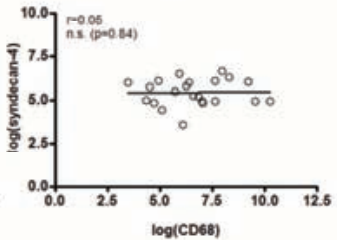

MMP-1 vs CD68

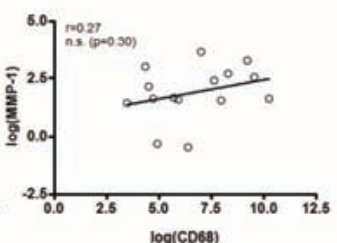

TIMP.2 vs CD68

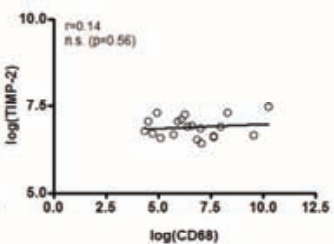

TIMP -4 vs CD68
B

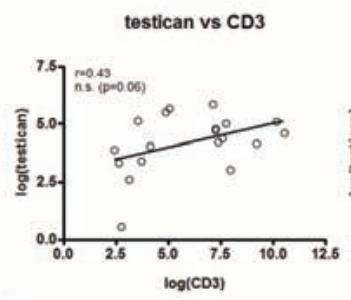

D

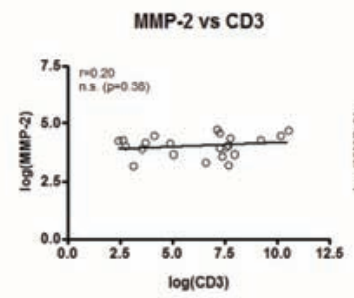

F

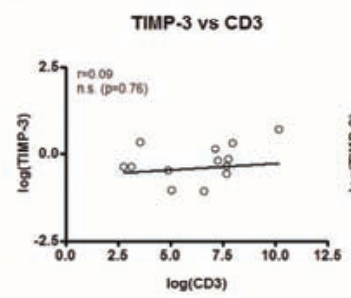

testican vs CD68

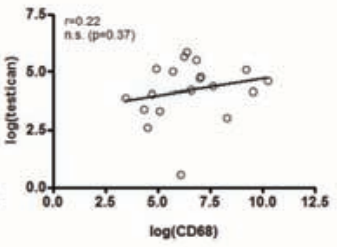

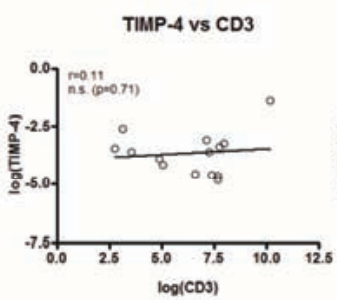

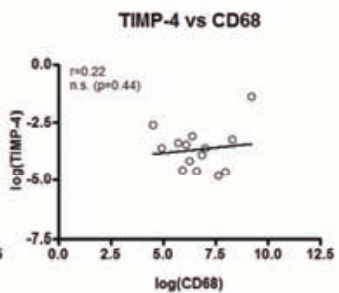

Supplemental figure 2. Intragraft transcript levels in relation to the influx of T-cells and monocytes in the transplanted heart. Intragraft transcript levels of (A) Syndecan-4, (B) Testican-1, (C) MMP-1, (D) MMP-2, (E) TIMP-2, (F) TIMP-3 and (G) TIMP-4 in relation to the influx of $C D 3^{+}$T-cells and CD68 monocytes. ( $n=102$, including $n=38$ for ISHLT grade $0, n=31$ for grade $1 A, n=25$ for grade $1 B$ and $n=8$ for grade 3 ). 
A

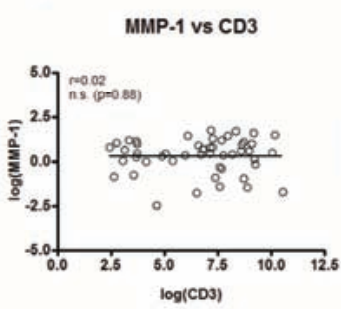

c

TIMP-1 vs CD3

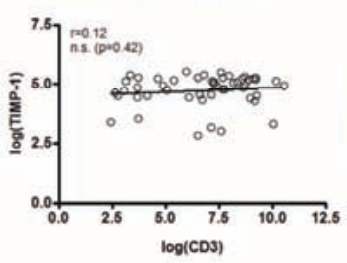

E

TIMP-3 vs CD3

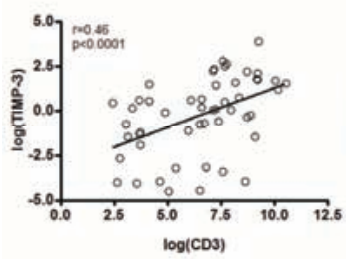

B

MMP-1 vs CD68
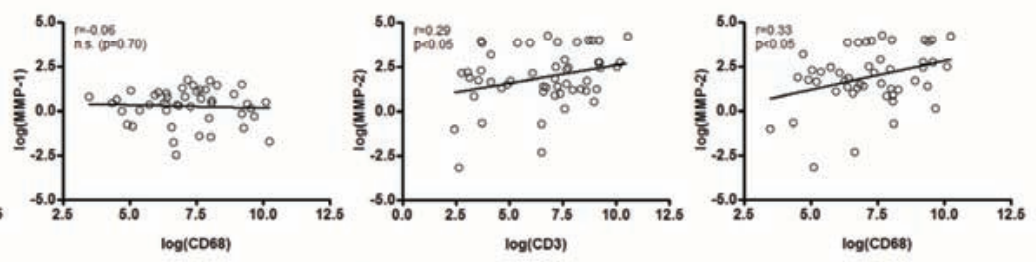

D

TIMP-1 vs CD68

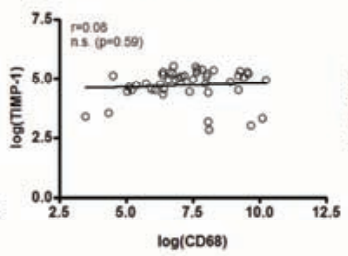

TIMP-2 vs CD3
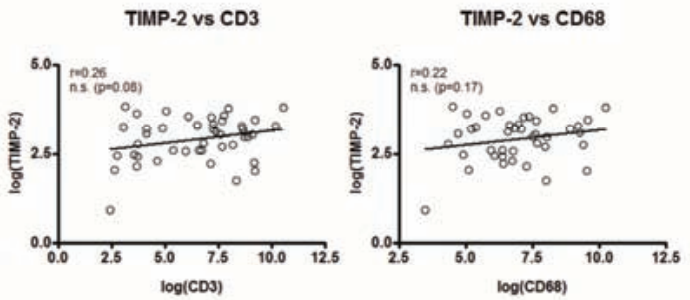

Supplemental figure 3. Intragraft MMP-1, -2, TIMP-1, -2 and -3 protein expression in relation to the influx of T-cells and monocytes in the transplanted heart. Intragraft protein expression of (A) MMP-1, (B) MMP-2, (C) TIMP-1, (D) TIMP-2 and (E) TIMP-3 protein expression levels in relation to the number of $C D 3^{+} \mathrm{T}_{\text {-cells }}$ and CD68 ${ }^{+}$monocytes. ( $n=64$, including $n=21$ for ISHLT grade $0, n=19$ for grade $1 A, n=16$ for grade $1 B$ and $n=8$ for grade 3). 

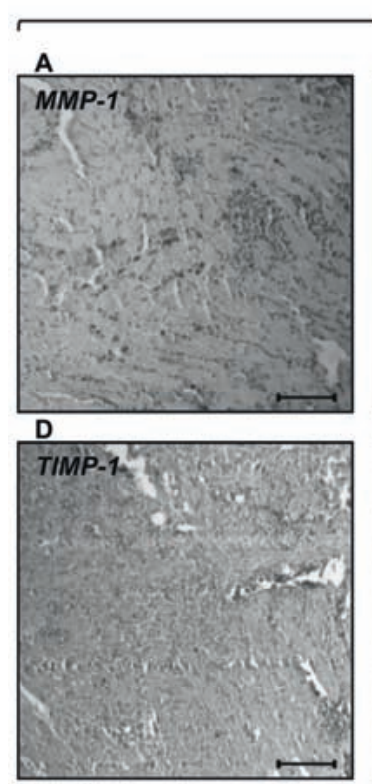

\section{ISHLT-Rejection Grade 3}

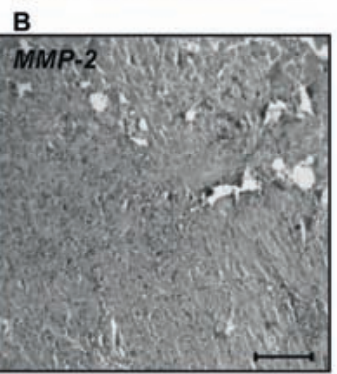

E
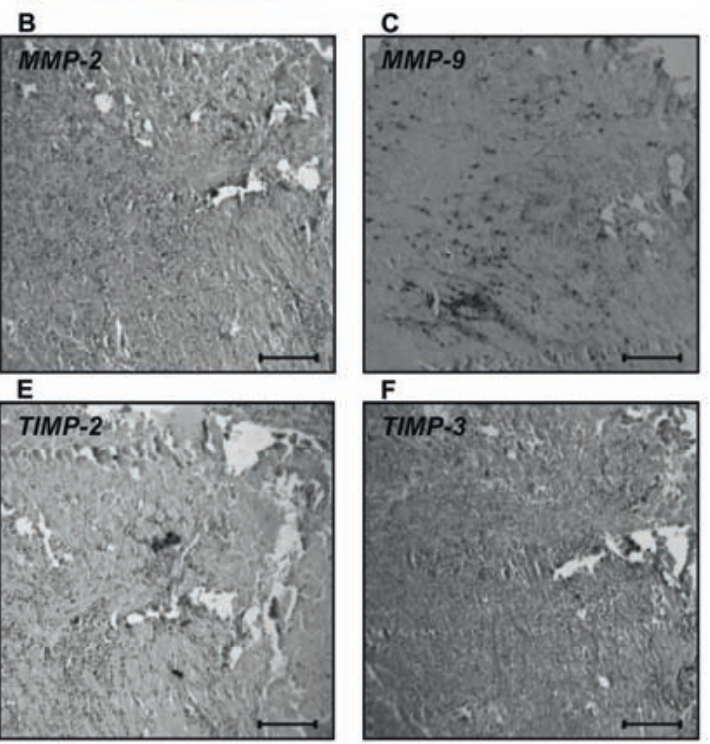

Supplemental figure 4. MMP/TIMP-immunoreactivity in endomyocardial biopsies after heart transplantation (HTX). Representative microphotographs of (A) MMP-1, (B) MMP-2, (C) MMP-9, (D) TIMP-1, (E) TIMP-2 and (F) TIMP-3 in grade 3 rejection EMBs. Scale-bar: $100 \mu \mathrm{m}$. 


\section{Supplemental references}

1 Heymans S, Schroen B, Vermeersch P, Milting H, Gao F, Kassner A, Gillijns H, Herijgers P, Flameng W, Carmeliet $P$, Van de Werf F, Pinto YM, Janssens $S$. Increased cardiac expression of tissue inhibitor of metalloproteinase- 1 and tissue inhibitor of metalloproteinase- 2 is related to cardiac fibrosis and dysfunction in the chronic pressure-overloaded human heart. Circulation. 2005;112(8):1136-1144.

2 Schellings MW, Vanhoutte D, Swinnen M, Cleutjens JP, Debets J, van Leeuwen RE, d'Hooge J, Van de Werf F, Carmeliet P, Pinto YM, Sage EH, Heymans S. Absence of SPARC results in increased cardiac rupture and dysfunction after acute myocardial infarction. J Exp Med. 2009;206(1):113-123.

3 Heymans S, Lupu F, Terclavers S, Vanwetswinkel B, Herbert JM, Baker A, Collen D, Carmeliet P, Moons L. Loss or inhibition of UPA or MMP-9 attenuates LV remodeling and dysfunction after acute pressure overload in mice. Am J Pathol. 2005;166(1):15-25.

4 Lehoux S, Lemarie CA, Esposito B, Lijnen HR, Tedgui A. Pressure-induced matrix metalloproteinase-9 contributes to early hypertensive remodeling. Circulation. 2004;109(8):1041-1047. 

Chapter 7

General discussion and conclusions 


\section{General discussion and conclusions}

Mechanisms that contribute to the development of heart failure have extensively been studied over the past decades, which lead to new and improved treatment strategies and increased our knowledge on cardiac pathophysiology. It is well accepted that factors such as increased workload and loss of cardiac cells induce myocardial remodeling which causes a change in cardiac size, shape, and function. ${ }^{1,2}$ From a molecular point of view these responses involve an extensive rearrangement of the cellular and extracellular matrix compartment, and depends on proper communication between cells and their surrounding ECM. ${ }^{3}$ The majority of the ECM consists of structural proteins such as collagens and elastin, which suggests an architectural role for the ECM as the 'cement' of the heart. ${ }^{4,5}$ Nonetheless, non-structural ECM components -also called matricellular proteins- have been implicated in regulating ECM turnover and cell behavior such as migration, proliferation and differentiation during wound repair, angiogenesis, and embryonic development. This implicates that the ECM not only acts as an architectural backbone that determines strength of the cardiac tissue, but also directs cardiac cell behavior in order to properly coordinate the remodeling response. This thesis focuses on the matricellular proteins thrombospondin-2 (TSP-2) (chapter 2-4), thrombospondin-1 (TSP-1) and connective tissue growth factor (CTGF) (chapter 5), and the matrix proteoglycan syndecan-1 and matrix metalloproteinase 9 (chapter 6), and discusses their role in the context of their pleiotropic function in cardiac remodeling; ie. how these matricellular molecules not only regulate ECM deposition and turnover but also direct cardiomyocyte behavior.

TSPs are highlighted as crucial regulators of wound repair and tumorigenesis. Increased expression of TSP-1 after myocardial infarction and enhanced TSP-2 levels in hypertensive heart disease and cardiac aging revealed a role of these matrix proteins in the heart as well. ${ }^{6,7}$ Nevertheless, their role in cardiac function remains largely unexplored. Chapter 2 elaborates the molecular characteristics of TSP-1 and TSP-2 that may relate to their role in cardiac function and remodeling. ${ }^{8}$ The cardioprotective effects of TSP-2 were further explored in a model of physiological aging and doxorubicininduced cardiomyopathy in mice, revealing that TSP-2 plays a central role in cardiomyocyte survival and matrix strengthening (chapter 3 and 4). ${ }^{7,9}$ Chapter 5 describes changes in the cardiac expression pattern of TSP-1 and CTGF related to heart failure prone compared to heart failure resistant aging, and reveals cardiomyocyte-specific regulation of these matrix molecules by microRNA (miRNA) -18 and $-19 .^{10}$ The role of non-structural matrix molecules and matrix metalloproteinases (MMPs) and their inhibitors (TIMPs) in inflammation and fibrotic remodeling was studied in human cardiac allograft rejection (CAR) and identified the proteoglycan syndecan-1 and MMP-9 to be strongly related to degree of CAR (chapter 6$)$. 
The studies described in this thesis support our hypothesis that the ECM is a highly dynamic, plastic structure that actively orchestrates cell function and behavior in cardiac function and disease and demonstrate the pleiotropic character of matricellular proteins in heart failure. Insight in the regulation and role of these non-structural ECM proteins is key in our understanding of cardiac remodeling and our attempts to develop new strategies to interfere with and orchestrate the cardiac response after injury.

\section{Matricellular proteins in the heart: implications in cardiomyocyte function}

The pleiotropic character of matricellular proteins is suggested in chapter 4 demonstrating that TSP-2 not only regulates the integrity of the ECM architecture, but also affects cell survival of cardiomyocytes in doxorubicin-induced cardiomyopathy. This pleiotropic character was further supported by the fact that TSP-2 affects ECM integrity and cardiomyocyte survival in a similar manner in cardiac aging in mice (chapter 3). ${ }^{7}$ Hence, other matricellular proteins such as CTGF and periostin have been implicated in ECM maintenance and directing cardiomyocyte behavior as well. CTGF acts downstream of TGF- $\beta$ and is well known for its profibrotic effects, predominantly via promotion of fibroblast proliferation and enhanced ECM deposition. ${ }^{11}$ However, stimulation of cardiomyocytes in vitro with full length CTGF or its C-terminal peptide induces a hypertrophic response in an Akt-dependent manner, indicating that expression of this profibrotic molecule also affects the cardiomyocyte compartment in the heart. ${ }^{12}$ Another matricellular protein, periostin, was implicated in regulation of interstitial fibrosis and hypertrophy following pressure overload and myocardial infarction in mice. ${ }^{13}$ Periostin was shown to regulate differentiation of progenitor mesenchymal cells into fibroblasts while on the other hand it induces cardiomyocyte hypertrophy. ${ }^{14}$, 15 Besides non-structural matrix proteins, fibronectin derived from embryonic fibroblasts was shown to direct cardiomyocyte proliferation during development in a paracrine fashion. ${ }^{16}$ Altogether, these studies suggest that the effects of matricellular proteins are not limited to ECM deposition and turnover, but also affect cardiomyocyte responses to injury; an additional function of these non-structural ECM molecules that is relatively less well defined.

\section{Cell type-specific matricellular protein production: functional consequences?}

Despite the pronounced cardiomyocyte effects of some matricellular proteins it is often poorly described which cell type is responsible for the altered expression observed following cardiac stress. Fibroblasts constitute more than $70 \%$ of all cardiac cells and about $90 \%$ of non-cardiomyocytes. ${ }^{17,} 18$ Therefore the dogma is that ECM molecules are mainly produced by cardiac fibroblasts. The results of chapter 4 imply that TSP-2 is expressed in both cell types, but its induction is differentially regulated in 
cardiac fibroblasts as compared to cardiomyocytes after doxorubicin exposure. ${ }^{9}$ Importantly, these differences in TSP-2 expression seem to affect distinct mechanisms in cardiac remodeling; i.e. increased TSP-2 expression in fibroblasts represses MMP-2 activity to maintain ECM integrity, whereas increased TSP-2 expression in cardiomyocytes promotes cell survival. These findings are in line with other studies that report cardiomyocyte-derived expression of matricellular proteins upon various stimuli. For example, Chen et al. demonstrated that TGF- $\beta$ not only induces CTGF expression in cardiac fibroblasts, but also in cardiomyocytes. ${ }^{19}$ Two other potent inducers of cardiac remodeling, phenylephrine (PE) and endothelin-1 (ET-1), enhanced CTGF transcription in cardiomyocytes in vitro as well. ${ }^{20,21}$ The biological relevance of cardiomyocytederived CTGF induction is suggested by the fact that CTGF expression is observed in cardiomyocytes residing in the infarct borderzone 2 days after injury. ${ }^{11}$ In addition, cardiomyocyte-specific TSP-2 expression can be abrogated via inhibition of Akt phosphorylation in vitro. ${ }^{7}$ Syndecan-1, a matrix proteoglycan, is upregulated in neonatal cardiomyocytes following oxidative stress caused by hydrogen peroxide ${ }^{22}$, whereas another proteoglycan, glypican, is almost exclusively expressed on the plasma membrane of cardiomyocytes during postnatal development. ${ }^{23}$ Altogether, these studies indicate that matricellular protein expression can be induced in cardiomyocytes.

The dogma that all matricellular proteins are secreted into the extracellular environment does not address the fact that these molecules can originate from different cell types within a tissue. Although matricellular proteins have different effects on cardiomyocytes compared to cardiac fibroblasts, it is not understood whether this is related to cell-specific secretion. I.e. most matricellular proteins are glycoproteins that have multiple post-translational modifications, which speculatively may differ according to their cellular origin. Moreover, it is unknown if exocytosis of matricellular proteins produced by cardiomyocytes occurs in a similar manner as in cardiac fibroblasts. For example, if cardiomyocyte-derived matricellular proteins are not secreted into the ECM, but remain at the site of cell membrane, the observed cellular response is predominantly triggered via a paracrine route. This might explain the differences in cellular responses observed in cardiomyocytes and cardiac fibroblasts, and implies that not only the target-cell type but also the source of matricellular protein production is of significant importance in regulating tissue remodeling. Nevertheless, in vivo studies are not always conclusive about the source of matricellular protein production, and it often remains elusive whether the observed cardiomyocyte effect is driven by fibroblast-derived matrix molecules or is a paracrine effect of local cardiomyocyte expression.

Cardiomyocyte-derived expression of matricellular proteins contributes to cardiac remodeling and function

In cardiac research, in vivo cardiac-specific manipulation of genes of interest occurs in the setting of the cardiomyocyte. The above paragraph summarizes a role for matricel- 
lular proteins in cardiac fibroblasts as well as cardiomyocytes, and hints to a difference in matricellular protein functionality depending on cellular source. Therefore, the interpretation of cardiomyocyte-restricted transgenic mouse models may be complicated by the possibility that these gene products do not reflect the physiological situation, in which most matricellular proteins derive from cardiac fibroblasts and are produced at sites of increased fibroblast activity and injury. As a first example, cardiomyocyte-restricted overexpression of CTGF (CTGF-TG) in murine and rat hearts does not induce cardiac fibrosis, but rather elicits a hypertrophic response. ${ }^{24}$ Fibrosis increases to a similar extent in CTGF-TG and wild-type mice upon angiotensin II (Ang II) infusion, but cardiac function was preserved in CTGF-overexpressing hearts suggesting a beneficial role during acute induction of hypertrophy. In addition CTGF-TG mice subjected to ischemia-reperfusion displayed a markedly diminished infarct size. ${ }^{25}$ However, on the long-term, non-stressed CTGF-TG animals develop age-related heart failure characterized by compensatory hypertrophy, dilatation and systolic dysfunction. These findings suggest that cardiomyocyte-derived CTGF protects against stress-induced cardiac dysfunction in an acute setting, while long-term upregulation leads to overstimulation and activation of pathological pathways that result in loss of cardiomyocytes and ventricular dilatation. ${ }^{24}$

Similar results are reported in a cardiac transgenic mouse model of the secreted matrix protein periostin, which is exclusively expressed in cardiac fibroblast after injury. ${ }^{15}$ Nevertheless, cardiomyocyte-restricted overexpression was shown to protect the heart from wall rupture following myocardial infarction (MI). On the long-term, overexpression promotes a mild hypertrophic response in aged mice, whereas mice that lack periostin are less susceptible to cardiac hypertrophy and fibrosis following long-term pressure overload and $\mathrm{MI}^{13}$ Furthermore, mindin (spondin-2), a secreted matricellular protein with C-terminal thrombospondin type 1 repeats, was shown to be downregulated in the ventricles of dilated cardiomyopathy patients with end-stage heart failure compared to donor hearts. ${ }^{26}$ Cardiomyocyte-specific mindinoverexpressing transgenic (mindin-TG) mice were protected against maladaptive hypertrophic remodeling following pressure overload and Ang II infusion, via blocking of the Akt/glycogen synthase kinase $3 \beta$ and TGF- $\beta /$ Smad singnaling in cardiomyocytes. ${ }^{26}$ Moreover, fibrosis was attenuated in Angll-treated mindin-TG mice, through abrogation of the response of profibrotic factors such as CTGF and TGF- $\beta$, also implying that cardiomyocyte-derived mindin expression affects both the fibroblast and myocyte compartment. ${ }^{26}$ Finally, biglycan, which belongs to the matricellular protein family of small leucine rich proteoglycans, has a cardioprotective role when overexpressed in the hearts of mice, as it activates TGF- $\beta$ and nitric oxide synthases. ${ }^{27}$ Importantly, all cardiomyocyte-restricted transgenic models discussed above show a protective role in an acute setting such as pressure overload or MI, whereas long-term overexpression leads to maladaptive remodeling and cardiac dysfunction. These observations support the therapeutic potential of short-term expression of matricellular proteins in the treatment of acute cardiac conditions such as $\mathrm{Ml}$ or pressure overload. However, it 
remains a challenging task to develop a strategy that allows site-directed delivery of matricellular proteins or 'on-demand' cardiomyocyte-specific expression which can be applied in acute heart failure.

\section{MiRNAs in the heart: implications in regulation of matricellular proteins}

Many studies have indicated that the adaptive and maladaptive remodeling response in the heart involve differential expression of non-structural ECM proteins. Matrix molecules are constantly expressed, as they contribute to tissue homeostasis, and therefore the changes in expression levels during pathological conditions might result from mechanisms that function to fine-tune their transcription.

Small non-coding miRNA molecules have gained an enormous interest in the field of genetic research as they form an endogenous mechanism that regulates gene expression in a post-transcriptional matter. The relevance of this mechanism in cardiovascular diseases was illustrated by gene expression profiling studies showing altered expression of specific miRNAs in failing human hearts ${ }^{28,29}$, and animal models that demonstrate their involvement in cardiac development and disease. ${ }^{30-32}$ Since the expression profile of certain miRNAs is strongly related to a specific tissue and disease, most studies focused on the miRNA families miR-1, miR-133a and miR-208 that are abundantly or uniquely expressed in the heart, and were found to be predominantly involved in regulation of the hypertrophic response. ${ }^{31,33-35}$ Although alterations in interstitial fibrosis were reported in these studies, all of them considered the enhanced ECM deposition to be secondary to cardiac hypertrophy and not under the direct control of miRNAs. Nevertheless, increasing evidence provides support for miRNAcontrolled regulation of fibrosis via direct targeting of ECM molecules.

\section{Regulation of cardiac fibrosis by miRNAs}

Increased interstitial fibrosis is not only observed in maladaptive hypertrophy but also in ischemic and dilated cardiac conditions. Moreover, fibrosis is observed in miRNAhypertrophy-related models, but the direct relation between specific miRNAs and cardiac fibrosis is less well defined. As matricellular proteins are generally known for their crucial role in ECM remodeling in the heart, it is tempting to speculate that regulation of such key-molecules by miRNAs contributes to cardiac fibrosis.

MiR-21 has recently been found to be strongly induced in heart failure. ${ }^{29,36}$ The effects of miR-21 are highly cell type-specific and seem to be restricted to cardiac fibroblasts. In cardiomyocytes miR-21 was shown to be crucial in the development of hypertrophy, however, at the same time also inhibitory effects of miR-21 were reported. ${ }^{37,38}$ Up-regulation of miR-21 in response to hypertrophy was shown to target sprouty homolog 1 in fibroblasts and relieves the ERK-MAP pathway, resulting in fibro- 
blast proliferation and fibrosis. ${ }^{36}$ In addition, upregulation of miR-21 following ischemia enabled enhanced matrix deposition through inhibition of MMP-2. ${ }^{39}$

Another miRNA that is preferentially expressed in fibroblasts compared to cardiomyocytes is miR-29. ${ }^{40}$ Importantly, miR-29 is one of the few miRNAs that directly regulate the expression of ECM components like collagens, fibrillins and elastins, and thereby contributes to the development of cardiac fibrosis. ${ }^{40}$ Other profibrotic genes, like osteonectin have also been identied as targets of miR-29, however their role in cardiac fibrosis is yet unknown. ${ }^{41}$

Chapter 4 describes the aging-associated changes in the expression of miRNA-18a and $-19 a / b$ to be related to altered expression profiles of the profibrotic matricellular proteins CTGF and TSP-1. Moreover, in aging-associated heart failure, decreased expression of miR-18a and miR-19a/b was linked to enhanced expression of their target genes CTGF and TSP-1 in vivo and in vitro in cardiomyocytes. In concordance, enhanced CTGF and TSP-1 levels correlated with increased cardiac fibrosis and heart failure in senescent mice (104 weeks old). Their role in regulating ECM deposition was further strengthened by the fact that repressing or enhancing CTGF and TSP-1 expression using miR-18a and miR-19b mimics (overexpression) and antagomirs (inhibition) respectively in cardiomyocytes, coincided with decreased or increased collagen type $1 \mathrm{~A}$ and $3 \mathrm{~A}$ transcription. Importantly, these miRNAs are much more abundantly expressed in cardiomyocytes compared to cardiac fibroblasts, which explains why this effect was restricted to cardiomyocytes and functions to fine-tune the expression of CTGF and TSP-1. This fine-tuning of CTGF protein levels by miRNAs in a cell type-specific manner was also described by Duisters et al., who showed that cardiomyocytes express miR133 and miR-30 to regulate CTGF expression, whereas fibroblasts mainly possess miR30 to inhibit CTGF expression. ${ }^{42}$ Evidence for the contribution of miR-133 and miR-30 to cardiac fibrosis was provided by the observation that their expression inversely correlated with collagen and fibrosis levels in patients with LV hypertrophy and in rodent models, in addition to the fact that miR-133 knockout mice suffer from severe fibrosis and heart failure. ${ }^{33,42}$ In concordance, cardiomyocyte-specific overexpression of miR-133 in mice did not enhance cardiac hypertrophy following transaortic constriction (TAC), but lead to an improvement of myocardial fibrosis and dysfunction. ${ }^{43}$

These examples demonstrate that miRNAs are indeed involved in the development of myocardial fibrosis. However, it is surprising that despite the fact that matricellular proteins are so often implicated in regulating cardiac remodeling and fibrosis, their regulation by miRNAs remains largely unexplored.

\section{Matricellular proteins and their predicted target-miRNAs}

The findings in this thesis are conform the increasing line of evidence that matricellular proteins play a crucial role in the process of cardiac remodeling. They are critically involved in cardiac hypertrophy, fibrosis, and inflammation and thereby strongly determine cardiac adaptation and function following injury. In addition, it is now recog- 
nized that miRNAs form a new level of transcriptional control that contributes to development of cardiac hypertrophy and fibrosis. However, the regulation of specific heart failure-related matricellular proteins by miRNAs gained little attention so far.

It is often difficult to assign a large biological response to the effect of one miRNA on a single gene target. Instead, the complexity of gene regulation by miRNAs lies within the fact that one miRNA can target different genes, whereas one gene can be regulated by multiple miRNAs. Therefore it is more likely that a group of miRNAs that are simultaneously expressed and targets different genes involved in one biological pathway, will have a larger effect.

Upregulation of the matricellular proteins TSP-1, TSP-2, CTGF, osteonectin, periostin, fibronectin and syndecan-1 has been implicated in heart failure. ${ }^{6,13,44-48}$ To enable a discussion on the possible role of microRNAs in the control of cardiac fibrosis and in the functioning of matricellular proteins, we explored 7 selected matricellular proteins for microRNA target sites using the web-based bioinformatical tool TargetScan (www.targetscan.org; release 5.1). Table 1 lists all the miRNAs with a predicted conserved target site in at least 1 matricellular protein. MiRNA target sites of the hypertrophy-related miR-1, miR-23a, and miR-208 were predicted in the 3'-UTR of only two matricellular protein genes, and miR-133 was predicted to target three genes. Two of the miRNAs that were described to be involved in cardiac fibrosis, miR-21 and miR-30, were predicted to target as little as two and one gene respectively. Mir-29, on the other hand, was predicted to target both TSP-1 and TSP-2, in addition to the highly conserved target-sites predicted in the transcripts of fibronectin and osteonectin. A review by Schroen et al. provides an overview of the expression profiles of different miRNAs reported in different models of heart failure. ${ }^{49}$ In this review miR-29 was summarized to be downregulated in human dilated cardiomyopathy ${ }^{50}$ and after TAC ${ }^{37}$, ${ }^{51}$ and $\mathrm{MI}^{52}$ in mice and, whereas its expression was found to be upregulated in heart failure patients. $^{39,53}$ Downregulation of miR-29 in acute cardiac injury might favor the formation of fibrosis following these events. However, it is unknown whether this is related miR-29-controlled expression of osteonectin, fibronectin, TSP-1 or TSP-2.

MiR-132 and miR-212 belong to the same family of miRNAs, and were predicted to target five out of seven analyzed matrix proteins; i.e. CTGF, fibronectin, periostin, TSP1, and TSP-2 (Table 1). These miRNAs are associated with development of the mammary glands in mice via direct targeting of MMP-9 ${ }^{54}$, however currently there are no reports on their role in the heart. Another miRNA-family consisting of miR-204 and miR-211, was also predicted to target five out of seven gene transcripts (Table 1), and was reported to regulate maintenance of the tight junctions in human retinal pigment epithelial cells via regulation of TGF- $\beta$ receptor $2 .^{55}$ Like miR-132/212, also miR$204 / 211$ is currently not implicated in cardiac functions. 
Table 1. Conserved miRNA target sites in the 3'-UTR of matricellular proteins

\begin{tabular}{|c|c|c|c|c|c|c|c|c|c|c|c|c|c|c|c|}
\hline & 嵌 & 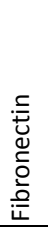 & 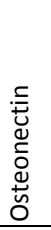 & 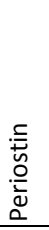 & 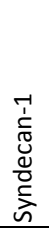 & 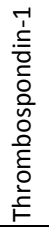 & 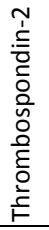 & & 岕 & 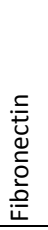 & 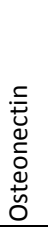 & 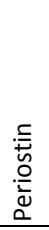 & 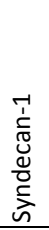 & 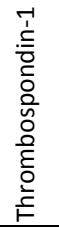 & 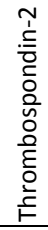 \\
\hline miR-1 & & & & & $x$ & $x$ & & miR-181 & $x$ & & & & & $x$ & $x$ \\
\hline miR-7ab & & $x$ & & & & $x$ & & $\operatorname{miR}-182$ & & $x$ & & & $x$ & $x$ & $x$ \\
\hline miR-9 & & $x$ & & & $x$ & & $x$ & miR-183 & $x$ & & & & & & \\
\hline miR-10 & & & & & $x$ & & & miR-190 & & $x$ & & & & & \\
\hline miR-15 & & & & & & & $x$ & miR-191 & & & & & $x$ & & \\
\hline miR-16 & & & & & & & $x$ & miR-192 & & & $x$ & & & & \\
\hline miR-17-5p & & & $x$ & & & & $x$ & miR-193ab & & & & & $x$ & & \\
\hline miR-18ab & $x$ & & $x$ & $x$ & & $x$ & & miR-194 & & $x$ & & & & $x$ & \\
\hline $\operatorname{miR}-19$ & $x$ & $x$ & $x$ & $x$ & $x$ & $x$ & & miR-195 & & & & & & & $x$ \\
\hline miR-20ab & & & $x$ & & & & $x$ & miR-199-5p & $x$ & & & & & & \\
\hline miR-22 & & & $x$ & & & & & miR-200a & & & & & & $x$ & \\
\hline miR-21 & & $x$ & & & & $x$ & $x$ & miR-203 & $x$ & $x$ & $x$ & & & & $\times$ \\
\hline miR-23ab & & $x$ & $x$ & & & & & miR-204 & & $x$ & $x$ & & $x$ & $x$ & $x$ \\
\hline miR-24 & & $x$ & & & & & & miR-205 & & $x$ & $x$ & & & $x$ & \\
\hline $\operatorname{miR}-25$ & & $x$ & & & & & & miR-206 & & & & & $x$ & $x$ & \\
\hline miR-26ab & $x$ & & & & & & & miR-208ab & & $x$ & & & & & $\times$ \\
\hline miR-27ab & & & & & $x$ & & $x$ & $\operatorname{miR}-210$ & & & & & & & $\times$ \\
\hline miR-29abc & & $x$ & $x$ & & & $x$ & $x$ & miR-211 & & $x$ & $x$ & & $x$ & $x$ & $\times$ \\
\hline miR-30abcde & & & & & & & $x$ & miR-212 & $x$ & $x$ & & $x$ & & $x$ & $\times$ \\
\hline miR-31 & & & $x$ & & $x$ & & $x$ & miR-215 & & & $x$ & & & & \\
\hline miR-32 & & $x$ & & & & & & miR-216ab & & $x$ & & & & & $\times$ \\
\hline miR-33ab & & & $x$ & $x$ & & & $x$ & miR-217 & & & & & & $x$ & $\times$ \\
\hline miR-34abc & & & $x$ & & $x$ & & & $\operatorname{miR}-218$ & $x$ & & & $x$ & & $x$ & \\
\hline miR-92ab & & $x$ & & & & & & miR-219-5p & & & $x$ & & & & \\
\hline miR-93 & & & $x$ & & & & $x$ & miR-221 & & & & & $x$ & $x$ & $\times$ \\
\hline miR-96 & & & & & & & $x$ & miR-222 & & & & & $x$ & $x$ & $\times$ \\
\hline miR-98 & & & & & & & & miR-302 & & & $\times$ & & $x$ & $x$ & $\times$ \\
\hline miR-101 & & $x$ & $x$ & & & $x$ & & miR-338-3p & & & & & $x$ & $x$ & \\
\hline miR-103 & & & & & & & $x$ & miR-351 & & & $x$ & & $x$ & & \\
\hline miR-106a & & & $x$ & & $x$ & $x$ & $x$ & miR-363 & & $x$ & & & & & \\
\hline miR-107 & & & & & & & $x$ & miR-367 & & $x$ & & & & & \\
\hline miR-122 & & & & & & & $x$ & miR-375 & $x$ & & & & & & $\times$ \\
\hline miR-124 & $x$ & & $x$ & & $x$ & & $x$ & miR-383 & $x$ & & & & & $x$ & \\
\hline miR-125 & & & $x$ & & $x$ & & & miR-384-5p & & & & & & & $\times$ \\
\hline
\end{tabular}




\begin{tabular}{|c|c|c|c|c|c|c|c|c|c|c|c|c|c|c|c|}
\hline & $\begin{array}{l}\text { 岕 } \\
\qquad\end{array}$ & 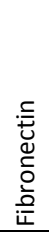 & 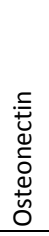 & 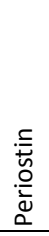 & 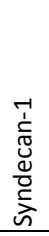 & 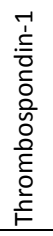 & 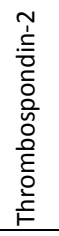 & & $\stackrel{U}{\leftrightarrows}$ & 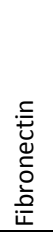 & 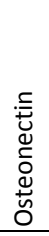 & 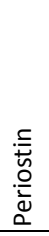 & 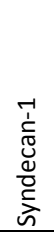 & 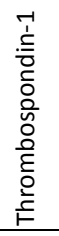 & 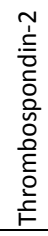 \\
\hline miR-132 & $x$ & $x$ & & $x$ & & $x$ & $x$ & miR-424 & & & & & & & $x$ \\
\hline miR-133 & $x$ & $x$ & & & $x$ & & & miR-425 & & $x$ & $x$ & $x$ & & & \\
\hline miR-135 & & & & $x$ & & $x$ & $x$ & miR-449abc & & & $x$ & & $x$ & & \\
\hline miR-138 & $x$ & & & & & $x$ & & $\operatorname{miR}-455$ & & & $x$ & & & & \\
\hline miR-139-5p & & & & & $x$ & $x$ & & miR-489 & & $x$ & $x$ & $x$ & & & \\
\hline miR-140 & & $x$ & & & & & & miR-490 & & & & & $x$ & & \\
\hline miR-141 & & & & & & $x$ & & miR-497 & & & & & & & $x$ \\
\hline miR-143 & $x$ & & & & & $x$ & & miR-499-5p & & $x$ & $x$ & & & & $x$ \\
\hline miR-144 & & $x$ & & & & $x$ & $x$ & miR-506 & $x$ & & $x$ & & $x$ & & $x$ \\
\hline miR-145 & $x$ & & & & & & & miR-519d & & & $x$ & & & & $x$ \\
\hline miR-146 & & & $x$ & & & & & miR-590-5p & & $x$ & & & & $x$ & $x$ \\
\hline miR-148 & & $x$ & & & & & & miR-699 & & & $x$ & & $x$ & & \\
\hline miR-150 & & & $x$ & & & & & miR-876-3p & & $x$ & & & & & \\
\hline miR-152 & & $x$ & & & & & & miR-1271 & & & & & & & $\times$ \\
\hline miR-155 & & & $x$ & & & & & miR-1297 & $x$ & & & & & & \\
\hline
\end{tabular}

Importantly, the target sites of miR-19 were broadly conserved in fibronectin, periostin and osteonectin and highly conserved in the transcripts of CTGF, syndecan-1 and TSP1 , and is thereby predicted to target most matricellular proteins of all miRNAs listed in Table 2. Chapter 4 discusses the role of miR-19 and its related cluster-member miR-18 in regulation of TSP-1 and CTGF levels in the aged heart. As previously discussed (chapter 5) miR-19 belongs to the miRNA-17 92 cluster (miR-17, -18a, 19a, -20a, -19b, and 92a) which is implicated in tumor formation and vascular biology. ${ }^{56,57}$ MiR-18 and -19 where predominantly linked to the downregulation of the anti-angiogenic CTGF and TSP-1, whereas another cluster member, miR-17, is known to regulate the expression of fibronectin related to cell adhesion, migration and proliferation. ${ }^{58}$ In addition, miR17 and miR-20a were reported to target TGF- $\beta$ receptor $2 .^{59}$ Interestingly, Dews et al. recently identified the miR-17 92 cluster to control expression levels of thrombospondin type I repeat (TSRs)-containing proteins, like TSP-1 and CTGF, and interfere with TGF- $\beta$ signaling through direct targeting of Smad2, Smad4 and TGF- $\beta$ receptor $2 .^{60,61}$ These studies revealed that upregulation of this cluster abrogates TGF- $\beta$ signaling, resulting in increased tumor cell growth. TGF- $\beta$ is recognized as an important cytokine, often implicated in cardiac remodeling and fibrosis. The fact that down regulation of miR-17 92 in age-related cardiac failure could be related to enhanced expression of CTGF, TSP-1 and collagen expression, supports that this cluster possibly acts to control 
TGF- $\beta$-related remodeling in the heart. Nevertheless, the role of this miRNA cluster or its individual cluster-members in cardiac function is currently unexplored.

In conclusion, this in silico approach has put forward a number of interesting miRNA candidates for exploration of their involvement in cardiac fibrosis and function via their regulation of matricellular proteins. The therapeutic possibilities of miRNAs make them very attractive research subjects, and a deeper understanding of their regulatory role in cardiac matricellular protein functioning could lead to new treatment strategies to selectively allow the beneficial effects of short-term cardiomyocyte matricellular protein expression.

\section{Conclusions and future perspectives}

The findings in this thesis illustrate the pleiotropic character of the ECM and its nonstructural components, as these proteins are involved in a wide variety of events including maintenance of ECM integrity and matrix deposition (chapter 3 and 4), cellmatrix communication (chapter 3 and 4), age-associated cardiac remodeling (chapter 3 and 5), and inflammation and the resulting fibrosis (chapter 6). This thesis also demonstrates that the function of certain ECM proteins highly depends on the tissue and cell type and the nature of the pathological condition; i.e. ECM proteins can induce outward-inward signaling leading to cell differentiation, migration or proliferation, such as in angiogenesis, but also regulate the influx of inflammatory cells, and the deposition of new ECM material. In concordance, this spatio-temporal function is recapitulated by their tissue and cell specific regulation by miRNAs.

Together ECM proteins shape and define the extracellular landscape at any time and thereby directly affect the behavior of the cells that reside within the tissue. Their involvement in various pathological conditions, not only including the heart, qualifies them as good candidates for future studies to treat diseases. Future studies to unravel the role of these non-structural ECM molecules in cardiac function should particularly consider their spatio-temporal expression in order to increase the chance of identifying and developing new treatment strategies in heart failure. In addition, their pleiotropic character makes them good candidates for direct drug targeting to prevent cardiac fibrosis and hypertrophy; as chaperone molecules for specific drug delivery strategies; or to be used as a tool to orchestrate the desired cell behavior in cardiac tissue engineering. Furthermore, the discovery of miRNAs and the increasing evidence for their role in the heart, provide a new, relatively unexplored, level to study cardiac remodeling. In particular, the regulation of matricellular protein expression by miRNAs gained little attention so far and remains elusive. Altogether matricellular proteins and their miRNA-controlled expression are potential candidates for future disease treatment in cardiovascular research and deserve further investigation. 


\section{References}

1 Cohn JN. Critical review of heart failure: the role of left ventricular remodeling in the therapeutic response. Clin Cardiol. 1995;18(9 Suppl 4):IV4-12.

2 Cohn JN. Structural basis for heart failure. Ventricular remodeling and its pharmacological inhibition. Circulation. 1995;91(10):2504-2507.

3 Swynghedauw B. Molecular mechanisms of myocardial remodeling. Physiol Rev. 1999;79(1):215-262.

4 Brown RD, Ambler SK, Mitchell MD, Long CS. The cardiac fibroblast: therapeutic target in myocardial remodeling and failure. Annu Rev Pharmacol Toxicol. 2005;45:657-687.

5 Fedak PW, Verma S, Weisel RD, Li RK. Cardiac remodeling and failure From molecules to man (Part II). Cardiovasc Pathol. 2005;14(2):49-60.

6 Schroen B, Heymans S, Sharma U, Blankesteijn WM, Pokharel S, Cleutjens JP, Porter JG, Evelo CT, Duisters R, van Leeuwen RE, Janssen BJ, Debets JJ, Smits JF, Daemen MJ, Crijns HJ, Bornstein P, Pinto YM. Thrombospondin-2 is essential for myocardial matrix integrity: increased expression identifies failureprone cardiac hypertrophy. Circ Res. 2004;95(5):515-522.

7 Swinnen M, Vanhoutte D, Van Almen GC, Hamdani N, Schellings MW, D'Hooge J, Van der Velden J, Weaver MS, Sage EH, Bornstein P, Verheyen FK, VandenDriessche T, Chuah MK, Westermann D, Paulus WJ, Van de Werf F, Schroen B, Carmeliet P, Pinto YM, Heymans S. Absence of thrombospondin-2 causes age-related dilated cardiomyopathy. Circulation. 2009;120(16):1585-1597.

8 Schellings MW, van Almen GC, Sage EH, Heymans S. Thrombospondins in the heart: potential functions in cardiac remodeling. J Cell Commun Signal. 2009;3(3-4):201-213.

9 van Almen GC, Swinnen M, Carai P, Verhesen W, Cleutjens JP, D'Hooge J, Verheyen FK, Pinto YM, Schroen B, Carmeliet P, Heymans S. Absence of thrombospondin-2 increases cardiomyocyte damage and matrix disruption in doxorubicin-induced cardiomyopathy. J Mol Cell Cardiol. 2011.

10 van Almen GC, Verhesen W, van Leeuwen RE, van de Vrie $M$, Eurlings $C$, Schellings MW, Swinnen $M$, Cleutjens JP, van Zandvoort MA, Heymans S, Schroen B. MicroRNA-18 and microRNA-19 regulate CTGF and TSP-1 expression in age-related heart failure. Aging Cell. 2011.

11 Ohnishi H, Oka T, Kusachi S, Nakanishi T, Takeda K, Nakahama M, Doi M, Murakami T, Ninomiya Y, Takigawa M, Tsuji T. Increased expression of connective tissue growth factor in the infarct zone of experimentally induced myocardial infarction in rats. J Mol Cell Cardiol. 1998;30(11):2411-2422.

12 Hayata N, Fujio Y, Yamamoto Y, Iwakura T, Obana M, Takai M, Mohri T, Nonen S, Maeda M, Azuma J. Connective tissue growth factor induces cardiac hypertrophy through Akt signaling. Biochem Biophys Res Commun. 2008;370(2):274-278.

13 Oka T, Xu J, Kaiser RA, Melendez J, Hambleton M, Sargent MA, Lorts A, Brunskill EW, Dorn GW, 2nd, Conway SJ, Aronow BJ, Robbins J, Molkentin JD. Genetic manipulation of periostin expression reveals a role in cardiac hypertrophy and ventricular remodeling. Circ Res. 2007;101(3):313-321.

14 Norris RA, Borg TK, Butcher JT, Baudino TA, Banerjee I, Markwald RR. Neonatal and adult cardiovascular pathophysiological remodeling and repair: developmental role of periostin. Ann N Y Acad Sci. 2008;1123:30-40.

15 Shimazaki M, Nakamura K, Kii I, Kashima T, Amizuka N, Li M, Saito M, Fukuda K, Nishiyama T, Kitajima S, Saga $Y$, Fukayama M, Sata M, Kudo A. Periostin is essential for cardiac healing after acute myocardial infarction. J Exp Med. 2008;205(2):295-303.

16 leda M, Tsuchihashi T, Ivey KN, Ross RS, Hong TT, Shaw RM, Srivastava D. Cardiac fibroblasts regulate myocardial proliferation through beta1 integrin signaling. Dev Cell. 2009;16(2):233-244.

17 Eghbali M, Blumenfeld OO, Seifter S, Buttrick PM, Leinwand LA, Robinson TF, Zern MA, Giambrone MA. Localization of types I, III and IV collagen mRNAs in rat heart cells by in situ hybridization. J Mol Cell Cardiol. 1989;21(1):103-113.

18 Eghbali M, Czaja MJ, Zeydel M, Weiner FR, Zern MA, Seifter S, Blumenfeld OO. Collagen chain mRNAs in isolated heart cells from young and adult rats. J Mol Cell Cardiol. 1988;20(3):267-276.

19 Chen MM, Lam A, Abraham JA, Schreiner GF, Joly AH. CTGF expression is induced by TGF- beta in cardiac fibroblasts and cardiac myocytes: a potential role in heart fibrosis. J Mol Cell Cardiol. 2000;32(10):18051819. 
20 Kemp TJ, Aggeli IK, Sugden PH, Clerk A. Phenylephrine and endothelin-1 upregulate connective tissue growth factor in neonatal rat cardiac myocytes. J Mol Cell Cardiol. 2004;37(2):603-606.

21 Recchia AG, Filice E, Pellegrino D, Dobrina A, Cerra MC, Maggiolini M. Endothelin-1 induces connective tissue growth factor expression in cardiomyocytes. J Mol Cell Cardiol. 2009;46(3):352-359.

22 Kemp TJ, Causton HC, Clerk A. Changes in gene expression induced by $\mathrm{H}(2) \mathrm{O}(2)$ in cardiac myocytes. Biochem Biophys Res Commun. 2003;307(2):416-421.

23 Asundi VK, Keister BF, Stahl RC, Carey DJ. Developmental and cell-type-specific expression of cell surface heparan sulfate proteoglycans in the rat heart. Exp Cell Res. 1997;230(1):145-153.

24 Panek AN, Posch MG, Alenina N, Ghadge SK, Erdmann B, Popova E, Perrot A, Geier C, Dietz R, Morano I, Bader M, Ozcelik C. Connective tissue growth factor overexpression in cardiomyocytes promotes cardiac hypertrophy and protection against pressure overload. PLoS One. 2009;4(8):e6743.

25 Ahmed MS, Gravning J, Martinov VN, von Lueder TG, Edvardsen T, Czibik G, Moe IT, Vinge LE, Oie E, Valen G, Attramadal H. Mechanisms of novel cardioprotective functions of CCN2/CTGF in myocardial ischemia-reperfusion injury. Am J Physiol Heart Circ Physiol. 2011;300(4):H1291-1302.

26 Yan L, Wei X, Tang QZ, Feng J, Zhang Y, Liu C, Bian ZY, Zhang LF, Chen M, Bai X, Wang AB, Fassett J, Chen Y, He YW, Yang Q, Liu PP, Li H. Cardiac-specific mindin overexpression attenuates cardiac hypertrophy via blocking AKT/GSK3\{beta\} and TGF-\{beta\}1-Smad signaling. Cardiovasc Res. 2011.

27 Bereczki E, Gonda S, Csont T, Korpos E, Zvara A, Ferdinandy P, Santha M. Overexpression of biglycan in the heart of transgenic mice: an antibody microarray study. J Proteome Res. 2007;6(2):854-861.

28 Thum T, Galuppo P, Wolf C, Fiedler J, Kneitz S, van Laake LW, Doevendans PA, Mummery CL, Borlak J, Haverich A, Gross C, Engelhardt S, Ertl G, Bauersachs J. MicroRNAs in the human heart: a clue to fetal gene reprogramming in heart failure. Circulation. 2007;116(3):258-267.

29 van Rooij E, Sutherland LB, Liu N, Williams AH, McAnally J, Gerard RD, Richardson JA, Olson EN. A signature pattern of stress-responsive microRNAs that can evoke cardiac hypertrophy and heart failure. Proc Natl Acad Sci U S A. 2006;103(48):18255-18260.

30 da Costa Martins PA, Salic K, Gladka MM, Armand AS, Leptidis S, el Azzouzi H, Hansen A, Coenen-de Roo CJ, Bierhuizen MF, van der Nagel R, van Kuik J, de Weger R, de Bruin A, Condorelli G, Arbones ML, Eschenhagen T, De Windt LJ. MicroRNA-199b targets the nuclear kinase Dyrk1a in an auto-amplification loop promoting calcineurin/NFAT signalling. Nat Cell Biol. 2010;12(12):1220-1227.

31 van Rooij E, Sutherland LB, Qi X, Richardson JA, Hill J, Olson EN. Control of stress-dependent cardiac growth and gene expression by a microRNA. Science. 2007;316(5824):575-579.

32 Zhao Y, Ransom JF, Li A, Vedantham V, von Drehle M, Muth AN, Tsuchihashi T, McManus MT, Schwartz RJ, Srivastava D. Dysregulation of cardiogenesis, cardiac conduction, and cell cycle in mice lacking miRNA-1-2. Cell. 2007;129(2):303-317.

33 Liu N, Bezprozvannaya S, Williams AH, Qi X, Richardson JA, Bassel-Duby R, Olson EN. microRNA-133a regulates cardiomyocyte proliferation and suppresses smooth muscle gene expression in the heart. Genes Dev. 2008;22(23):3242-3254.

34 Liu N, Williams AH, Kim Y, McAnally J, Bezprozvannaya S, Sutherland LB, Richardson JA, Bassel-Duby R, Olson EN. An intragenic MEF2-dependent enhancer directs muscle-specific expression of microRNAs 1 and 133. Proc Natl Acad Sci U S A. 2007;104(52):20844-20849.

35 Zhao $\mathrm{Y}$, Samal E, Srivastava D. Serum response factor regulates a muscle-specific microRNA that targets Hand2 during cardiogenesis. Nature. 2005;436(7048):214-220.

36 Thum T, Gross C, Fiedler J, Fischer T, Kissler S, Bussen M, Galuppo P, Just S, Rottbauer W, Frantz S, Castoldi M, Soutschek J, Koteliansky V, Rosenwald A, Basson MA, Licht JD, Pena JT, Rouhanifard SH, Muckenthaler MU, Tuschl T, Martin GR, Bauersachs J, Engelhardt S. MicroRNA-21 contributes to myocardial disease by stimulating MAP kinase signalling in fibroblasts. Nature. 2008;456(7224):980-984.

37 Cheng Y, Ji R, Yue J, Yang J, Liu X, Chen H, Dean DB, Zhang C. MicroRNAs are aberrantly expressed in hypertrophic heart: do they play a role in cardiac hypertrophy? Am J Pathol. 2007;170(6):1831-1840.

38 Tatsuguchi M, Seok HY, Callis TE, Thomson JM, Chen JF, Newman M, Rojas M, Hammond SM, Wang DZ. Expression of microRNAs is dynamically regulated during cardiomyocyte hypertrophy. J Mol Cell Cardiol. 2007;42(6):1137-1141. 
39 Roy S, Khanna S, Hussain SR, Biswas S, Azad A, Rink C, Gnyawali S, Shilo S, Nuovo GJ, Sen CK. MicroRNA expression in response to murine myocardial infarction: miR-21 regulates fibroblast metalloprotease-2 via phosphatase and tensin homologue. Cardiovasc Res. 2009;82(1):21-29.

40 van Rooij E, Sutherland LB, Thatcher JE, DiMaio JM, Naseem RH, Marshall WS, Hill JA, Olson EN. Dysregulation of microRNAs after myocardial infarction reveals a role of miR-29 in cardiac fibrosis. Proc Natl Acad Sci U S A. 2008;105(35):13027-13032.

41 Kapinas K, Kessler CB, Delany AM. miR-29 suppression of osteonectin in osteoblasts: regulation during differentiation and by canonical Wnt signaling. J Cell Biochem. 2009;108(1):216-224.

42 Duisters RF, Tijsen AJ, Schroen B, Leenders JJ, Lentink V, van der Made I, Herias V, van Leeuwen RE, Schellings MW, Barenbrug P, Maessen JG, Heymans S, Pinto YM, Creemers EE. miR-133 and miR-30 regulate connective tissue growth factor: implications for a role of microRNAs in myocardial matrix remodeling. Circ Res. 2009;104(2):170-178, 176p following 178.

43 Matkovich SJ, Wang W, Tu Y, Eschenbacher WH, Dorn LE, Condorelli G, Diwan A, Nerbonne JM, Dorn GW, 2nd. MicroRNA-133a protects against myocardial fibrosis and modulates electrical repolarization without affecting hypertrophy in pressure-overloaded adult hearts. Circ Res. 2009;106(1):166-175.

44 Frangogiannis NG, Ren G, Dewald O, Zymek P, Haudek S, Koerting A, Winkelmann K, Michael LH, Lawler J, Entman ML. Critical role of endogenous thrombospondin-1 in preventing expansion of healing myocardial infarcts. Circulation. 2005;111(22):2935-2942.

45 Koitabashi N, Arai M, Kogure S, Niwano K, Watanabe A, Aoki Y, Maeno T, Nishida T, Kubota S, Takigawa $\mathrm{M}$, Kurabayashi M. Increased connective tissue growth factor relative to brain natriuretic peptide as a determinant of myocardial fibrosis. Hypertension. 2007;49(5):1120-1127.

46 Schellings MW, Vanhoutte D, Swinnen M, Cleutjens JP, Debets J, van Leeuwen RE, d'Hooge J, Van de Werf F, Carmeliet P, Pinto YM, Sage EH, Heymans S. Absence of SPARC results in increased cardiac rupture and dysfunction after acute myocardial infarction. J Exp Med. 2009;206(1):113-123.

47 Vanhoutte D, Schellings MW, Gotte M, Swinnen M, Herias V, Wild MK, Vestweber D, Chorianopoulos E, Cortes V, Rigotti A, Stepp MA, Van de Werf F, Carmeliet P, Pinto YM, Heymans S. Increased expression of syndecan-1 protects against cardiac dilatation and dysfunction after myocardial infarction. Circulation. 2007;115(4):475-482.

48 Burgess ML, McCrea JC, Hedrick HL. Age-associated changes in cardiac matrix and integrins. Mech Ageing Dev. 2001;122(15):1739-1756.

49 Schroen B, Heymans S. MicroRNAs and beyond: the heart reveals its treasures. Hypertension. 2009;54(6):1189-1194.

50 Sucharov C, Bristow MR, Port JD. miRNA expression in the failing human heart: functional correlates. J Mol Cell Cardiol. 2008;45(2):185-192.

51 Lu Y, Zhang Y, Shan H, Pan Z, Li X, Li B, Xu C, Zhang B, Zhang F, Dong D, Song W, Qiao G, Yang B. MicroRNA-1 downregulation by propranolol in a rat model of myocardial infarction: a new mechanism for ischaemic cardioprotection. Cardiovasc Res. 2009;84(3):434-441.

52 Ikeda S, He A, Kong SW, Lu J, Bejar R, Bodyak N, Lee KH, Ma Q, Kang PM, Golub TR, Pu WT. MicroRNA-1 negatively regulates expression of the hypertrophy-associated calmodulin and Mef2a genes. Mol Cell Biol. 2009;29(8):2193-2204.

53 Ikeda S, Kong SW, Lu J, Bisping E, Zhang H, Allen PD, Golub TR, Pieske B, Pu WT. Altered microRNA expression in human heart disease. Physiol Genomics. 2007;31(3):367-373.

54 Ucar A, Vafaizadeh V, Jarry H, Fiedler J, Klemmt PA, Thum T, Groner B, Chowdhury K. miR-212 and miR132 are required for epithelial stromal interactions necessary for mouse mammary gland development. Nat Genet. 2010;42(12):1101-1108.

55 Wang FE, Zhang C, Maminishkis A, Dong L, Zhi C, Li R, Zhao J, Majerciak V, Gaur AB, Chen S, Miller SS. MicroRNA-204/211 alters epithelial physiology. FASEB J. 2010;24(5):1552-1571.

56 Dews M, Homayouni A, Yu D, Murphy D, Sevignani C, Wentzel E, Furth EE, Lee WM, Enders GH, Mendell JT, Thomas-Tikhonenko A. Augmentation of tumor angiogenesis by a Myc-activated microRNA cluster. Nat Genet. 2006;38(9):1060-1065.

57 Sen CK, Gordillo GM, Khanna S, Roy S. Micromanaging vascular biology: tiny microRNAs play big band. J Vasc Res. 2009;46(6):527-540. 
58 Shan SW, Lee DY, Deng Z, Shatseva T, Jeyapalan Z, Du WW, Zhang Y, Xuan JW, Yee SP, Siragam V, Yang BB. MicroRNA MiR-17 retards tissue growth and represses fibronectin expression. Nat Cell Biol. 2009;11(8):1031-1038.

59 Volinia S, Calin GA, Liu CG, Ambs S, Cimmino A, Petrocca F, Visone R, lorio M, Roldo C, Ferracin M, Prueitt RL, Yanaihara N, Lanza G, Scarpa A, Vecchione A, Negrini M, Harris CC, Croce CM. A microRNA expression signature of human solid tumors defines cancer gene targets. Proc Natl Acad Sci U S A. 2006;103(7):2257-2261.

60 Dews M, Fox JL, Hultine S, Sundaram P, Wang W, Liu YY, Furth E, Enders GH, El-Deiry W, Schelter JM, Cleary MA, Thomas-Tikhonenko A. The myc-miR-17 92 axis blunts TGF\{beta\} signaling and production of multiple TGF\{beta\}-dependent antiangiogenic factors. Cancer Res. 2010;70(20):8233-8246.

61 Mestdagh P, Bostrom AK, Impens F, Fredlund E, Van Peer G, De Antonellis P, von Stedingk K, Ghesquiere B, Schulte S, Dews M, Thomas-Tikhonenko A, Schulte JH, Zollo M, Schramm A, Gevaert K, Axelson H, Speleman F, Vandesompele J. The miR-17-92 microRNA cluster regulates multiple components of the TGF-beta pathway in neuroblastoma. Mol Cell. 2010;40(5):762-773. 



\section{Summary}

Cardiovascular diseases form a major health issue and its socio-economic burden will continue to rise over the next decade. Despite the improved survival rate of heart failure (HF) patients the incidence is still increasing, illustrating the need for better prevention and treatment strategies. HF often occurs as the result of maladaptive remodeling of the cardiac tissue following the heart's attempt to maintain cardiac function after injury. This response involves a substantial rearrangement of the cellular and extracellular matrix (ECM) compartment of the myocardium, and is tightly regulated by the interplay between cardiac cells and there surrounding matrix. Increasing evidence suggests that non-structural matricellular proteins not only regulate structural integrity of the ECM meshwork, but also play an indispensible role in cell-matrix communication and thereby determine cell and tissue function. This thesis hypothesizes that matricellular proteins not only regulate ECM deposition and turnover but also regulate cardiomyocyte behavior in the healthy and diseased heart and thus significantly affect the outcome of myocardial remodeling; i.e. preserved cardiac function (adaptive) or HF (maladaptive). The studies described here, highlight the matricellular proteins thrombospondin-1 (TSP-1) and -2 (TSP-2), connective tissue growth factor (CTGF), the proteoglycan syndecan-1 (synd-1) and matrix metalloproteinase-9 (MMP9), and extend the current knowledge on their role in the hypertensive and ischemic heart towards their function in cardiotoxicity, aging and cardiac allograft rejection.

Of the many matricellular proteins thrombospondins (TSPs) are highlighted as key modulators of angiogenesis, wound healing and tumor formation, but apart from enhanced expression associated with cardiac injury, little is known about their role in the heart. Chapter 2 elaborates on the function of TSPs in the heart, thereby focusing on their ability to interact with cytokines such as transforming growth factor $\beta$ (TGF- $\beta$ ), the cell surface receptors CD36 and CD47/integrin associated protein (CD47/IAP), and matrix metalloproteinases (MMPs) and their inhibitors (TIMPs). Despite the analogy in protein structure and thus their ability to interact with the same ligands, in particular of TSP-1 and TSP-2, TSPs have distinct functions depending on their spatio-temporal expression and the nature of the remodeling process; i.e. TSP-1 is associated with regulation of the early inflammatory response, whereas TSP-2 is related to proliferation and ECM maturation during later stages of remodeling after myocardial infarction (MI).

The pleiotropic character of TSP-2 is illustrated in chapter 3, revealing both ECM and cardiomyocyte abnormalities that contribute to accelerated cardiac aging in TSP-2 
knockout (TSP-2-KO) mice. Survival rate in the first 60 weeks of their lives was significantly lower in TSP-2-KO mice compared to wild-type (WT) littermates, as $>55 \%$ of the TSP-2-deficient mice died within 24-60 weeks. Surviving TSP-2-KO mice had increased cardiac dilation and fibrosis in addition to enhanced cardiomyocyte stress related to impaired Akt-survival signaling. Importantly, re-expression of TSP-2 in the hearts of TSP-2-KO mice normalized their survival and prevented cardiac dilation. Interestingly, doxorubicin (DOX) induced cardiotoxicity, as described in chapter 4, recapitulates the cardiac aging phenotype in TSP-2-KO mice. Twelve weeks of chronic DOX treatment causes substantial ECM disruption and cardiomyocyte apoptosis in TSP-2-KO hearts. Increased cell death after DOX is associated with reduced Akt phosphorylation in cardiomyocytes, similar to the cell stress observed in cardiac aging. Moreover, the enhanced matrix disruption in DOX-treated TSP-2-KO mice went along with increased MMP-2 levels. Together these studies show that TSP-2 affects both the ECM and cardiomyocyte compartment and thereby illustrate its bifunctional role in the heart.

Matricellular proteins are constantly expressed at relatively low levels during normal post-natal life in order to maintain tissue homeostasis, however during cardiac injury their expression increases. This suggests that changes in intrinsic mechanisms that function to fine-tune transcription of these proteins might result in altered expression profiles that affect the balance between ECM deposition and breakdown. Chapter 5 describes such a mechanism, and demonstrates that aging-associated changes in the expression of microRNA ( $\mathrm{miR}$ )-18a and $\mathrm{miR}-19 \mathrm{a} / \mathrm{b}$ relate to altered expression profiles of the profibrotic matricellular proteins CTGF and TSP-1. Aging-induced HF in 104 weeks old mice was characterized by decreased miR-18a and miR-19a/b expression paralleled by enhanced CTGF and TSP-1 protein levels, whereas opposite expression patterns were observed in age-matched mice with a preserved cardiac function. Importantly, this mechanism seems to be restricted to cardiomyocytes, as overexpression or inhibition of miR-18a and miR-19b, respectively blunts or enhanced CTGF and TSP-1 protein levels, coinciding with reduced or increased collagen expression, whereas repression of these miRNAs in fibroblasts had no effect on CTGF or TSP-1. This study implies that upregulation of miR-18a and miR-19a/b in cardiomyocytes functions to dampen the fibrotic remodeling process that contributes to the functional decline with cardiac aging.

Altered expression of matricellular proteins, proteoglycans and the MMP-system not only determines the mechanical properties of the heart, but also modulates key inflammatory and reparative mechanisms in models of cardiac ischemia, aging and myocarditis. Chapter 6 elaborates on the role of these ECM molecules in the inflammatory response in the transplanted heart. From a selection of inflammation-related matricellular proteins, proteoglycans and MMPs/TIMPs, synd-1 and MMP-9 were identified to mark the degree of cardiac allograft rejection. Enhanced expression of both synd-1 and MMP-9 correlates with the inflammatory markers tumor necrosis factor- $\alpha$ (TNF- $\alpha$ ) and 
interleukin-6 (IL-6), and parallels the progressive increase in $\mathrm{CD}^{+}{ }^{\text {T-cells }}$ and $\mathrm{CD} 68^{+}$ monocytes. Altogether suggesting that alterations in the expression of synd-1 and MMP-9 contribute to the pathogenesis of cardiac allograft rejection.

The versatility of roles of matricellular proteins described in this thesis illustrates their pleiotropic nature and strengthens the idea of the ECM as a highly dynamic meshwork of structural and non-structural molecules interacting with the embedded cells. Moreover, the function of certain matricellular proteins highly depends on the cell type and nature of the pathological condition. Therefore ECM proteins shape and define the extracellular landscape at any time and thereby directly affect the behavior of the cells that reside within the heart. Understanding of the pleiotropic nature in relation to the spatio-temporal expression of matricellular proteins provides new avenues for exploring their therapeutic potential. 



\section{Samenvatting}

Hart- en vaatziekten vormen een grote bedreiging voor de volksgezondheid en naar verwachting zal de omvang van dit probleem het komende decennium verder toenemen. Ondanks een stijging van de overlevingskans bij patiënten met hartfalen (HF) neemt het aantal nieuwe patiënten nog steeds toe en hiermee ook de behoefte aan verbeterde preventie en behandelmethoden. HF ontwikkelt zich als gevolg van maladaptieve remodellering van het hartweefsel na een uiterste poging van het hart om zijn functie te behouden na beschadiging. Tijdens deze reactie vindt een uitgebreide reorganisatie van de cellulaire en extracellulaire matrix (ECM) compartimenten plaats, die nauw wordt gereguleerd door het samenspel tussen de hartspiercellen en de omliggende matrix. Een toenemend aantal bevindingen impliceert dat niet-structurele, matricellulaire eiwitten niet alleen betrokken zijn bij de regulatie van de structurele eigenschappen van de ECM, maar ook een uiterst belangrijke rol spelen bij de communicatie tussen cellen en de ECM en zodoende cel- en weefselfunctie beïnvloeden. De hypothese in dit proefschrift stelt dat matricellulaire eiwitten niet alleen afbraak en opbouw van de ECM controleren, maar ook het gedrag van cardiomyocyten in het gezonde en zieke hart en daarmee het resultaat van cardiale remodellering beïnvloeden; dat wil zeggen, behoud van functie (adaptief) of de ontwikkeling van hartfalen (maladaptief). De studies in dit proefschrift belichten de matricellulaire eiwitten thrombospondin-1 (TSP-1) en -2 (TSP-2), connective tissue growth factor (CTGF), de proteoglycaan syndecan-1 (synd-1) en matrix metalloproteinase-9 (MMP-9) en behandelen hun rol in het hart na schade ten gevolge van cardiotoxicteit, veroudering en na harttransplantatie.

Van de vele matricellulaire eiwitten worden thrombospondines (TSPs) gezien als belangrijke modulatoren van angiogenese, wondheling en tumorvorming. Naast een verhoogde expressie bij beschadiging van het myocard, is weinig bekend over de rol van TSPs in het hart. Hoofdstuk 2 beschrijft de mogelijke functies van TSPs in het hart als gevolg van hun interactie met cytokinen zoals transforming growth factor- $\beta$ (TGF$\beta)$, oppervlakte receptoren als CD36 en CD47/integrin associated protein (CD47/IAP) en matrix metalloproteinases (MMPs) en hun inhibitoren (TIMPs). Ondanks de analogie in eiwitstructuur en daarmee de mogelijkheid om dezelfde liganden te binden is de functie van met name TSP-1 en TSP-2 sterk afhankelijk van hun plaats- en tijdsgebonden expressie en de aard van het remodelleringsproces; met name TSP-1 wordt geassocieerd met regulatie van de acute ontstekingsfase terwijl TSP-2 betrokken is bij de regulatie van celproliferatie en organisatie van de ECM gedurende latere fasen van remodellering na een hartinfarct. 
Het pleiotrope karakter van TSP-2 wordt verder beschreven in hoofdstuk 3 dat aantoont dat afwezigheid van TSP-2 in TSP-2 knockout (KO) muizen leidt tot veranderingen in de ECM structuur en cardiomyocytfunctie, die bijdragen aan de versnelde veroudering van de hartspier. Het aantal TSP-2-KO muizen dat de eerste 60 levensweken overleeft is ruim de helft lager dan in wildtype (WT) muizen, en mortaliteit treedt op vanaf een leeftijd van 24 weken. Overlevende TSP-2-KO muizen hebben een gedilateerd hart met de vorming van fibrose, naast verhoogde celschade als gevolg van verminderde Akt-survival signalering. Herstel van TSP-2 expressie in het hart van TSP-2-KO muizen voorkomt vroegtijdig overlijden en dilatatie van het myocard. Cardiotoxiciteit als gevolg van doxorubicine (DOX) behandeling, zoals beschreven in hoofdstuk 4, versnelt het fenotype van cardiale veroudering in TSP-2-KO muizen. Chronische behandeling voor 12 weken met DOX veroorzaakt een substantiële verstoring van de ECM organisatie en apoptose van cardiomyocyten in TSP-2-KO harten. Verhoogde celdood na DOX is gerelateerd aan verminderde Akt fosforylatie in cardiomyocyten, zoals reeds gezien in hartspiercellen tijdens veroudering. De sterk verminderde organisatie van de ECM in DOX behandelde TSP-2-KO muizen gaat gepaard met een verhoogde aanwezigheid van MMP-2. Deze studies tonen aan dat TSP-2 zowel de ECM als het cardiomyocyt compartiment beïnvloedt en bevestigen daarmee de bifunctionele rol van dit eiwit in het hart.

Expressie van matricellulaire eiwitten wordt gekenmerkt door de relatief lage aanwezigheid in gezond hartweefsel na de geboorte. Echter, tijdens beschadiging van de hartspier neemt de expressie toe. Dit suggereert dat kleine veranderingen binnen het mechanisme dat de transcriptie van deze eiwitten reguleert kan leiden tot een verschuiving van de balans tussen ECM opbouw en afbraak. Hoofdstuk 5 beschrijft hoe veranderingen in expressie van microRNA (miR)-18a en miR-19a/b tijdens veroudering gerelateerd zijn aan het expressie patroon van de profibrotische matricellulaire eiwitten CTGF en TSP-1. Muizen van 104 weken oud met HF worden gekenmerkt door een afname van miR-18a en miR-19a/b expressie tezamen met een toename van CTGF en TSP-1. Een omgekeerd expressie patroon is gevonden in muizen van dezelfde leeftijd waarbij de functie van het hart behouden was. Verhoogde inductie of remming van miR-18a en miR-19b in cardiomyocyten leidt tot een afname, respectievelijk toename in CTGF en TSP-1 expressie parallel met een verlaagde en verhoogde transcriptie van collagenen. Repressie van deze miRNAs in fibroblasten heeft echter geen effect op de expressie van CTGF en TSP-1, wat suggereert dat dit mechanisme specifiek is voor de cardiomyocyt. Deze studie impliceert dat miR-18a en miR-19b expressie wordt geïnduceerd in cardiomyocyten om het fibrotische remodelleringsproces in het hart te remmen en daarmee een vermindering van de hartfunctie tijdens veroudering te voorkomen.

Verandering in de expressie van matricellulaire eiwitten, proteoglycanen en het MMPsysteem beïnvloeden niet alleen de mechanische eigenschappen van het hart, maar spelen ook een belangrijke rol tijdens inflammatie in modellen van cardiale ischemie, 
veroudering en myocarditis. In hoofdstuk 6 wordt de rol van deze ECM moleculen besproken tijdens de inflammatoire reactie in het hart na transplantatie. Uit een screening van inflammatie-gerelateerde matricellulaire eiwitten, proteoglycanen en MMPs/TIMPs is naar voren gekomen dat het expressie patroon van synd-1 en MMP-9 representatief is voor de mate van afstoting van het getransplanteerde hart. Verhoogde expressie van synd-1 en MMP-9 correleert met een toename van de inflammatoire cytokinen tumor necrosis factor- $\alpha$ (TNF- $\alpha$ ) en interleukin-6 (IL-6) en met de aanwezigheid van T-cellen en monocyten. Deze bevindingen tonen aan dat verhoogde expressie van synd-1 en MMP-9 mogelijk een integrale rol speelt bij de pathogenese tijdens afstoting van het getransplanteerde hart.

De variëteit aan functies die beschreven worden in dit proefschrift illustreren het pleiotrope karakter van matricellulaire eiwitten en versterken de hypothese dat de ECM een complex netwerk vormt van structurele en niet-structurele eiwitten dat nauw communiceert met de cellen in het weefsel. Daarnaast wordt de functie van veel matricellulaire eiwitten bepaald door het celtype en de aard van de pathologische conditie. Het palet van aanwezige matricellulaire eiwitten is dus bepalend voor de eigenschappen van het extracellulaire landschap en beïnvloedt hiermee direct het gedrag van de aanwezige cellen in het hart. Begrip en herkenning van het pleiotrope karakter van matricellulaire eiwitten in relatie tot hun plaats- en tijdsgebonden expressie dragen bij aan de ontdekking van nieuwe therapeutische mogelijkheden van deze moleculen. 



\section{Dankwoord}

De laatste loodjes van dit proefschrift zijn voor het dankwoord: als laatste geschreven, het vaakst gelezen, maar bovenal het meest kritisch beoordeeld door iedereen die de afgelopen jaren een bijdrage heeft geleverd aan mijn promotie. Daarom wil ik dit dankwoord graag beginnen met een hele "dikke dankjewel" voor alle collega's, vrienden en familie. Zonder jullie hulp en oprechte interesse had dit proefschrift er nooit gelegen.

De volgende mensen wil ik graag in het bijzonder bedanken voor hun rol de afgelopen jaren.

Professor, dr. Heymans, beste Stephane. Toen ik als promovendus begon bij de groep was de rolverdeling anders dan nu. Uiteraard was mij over het bestaan van de extracellulaire matrix verteld, maar mijn interesse ging uit naar de moleculaire biologie die veroudering van de hartspiercellen veroorzaakt. Op het moment dat de groep van koers wijzigde en ik sterk twijfelde over het voortzetten van mijn promotie, was één gesprek genoeg om mij te overtuigen. We zouden de accenten van mijn onderzoek iets verleggen, een aantal nieuwe projecten starten en er mocht wel wat meer 'extracellulaire matrix' in. Tijdens onze werkbesprekingen zei u vaak dat ik "korter op de bal moest spelen". Mijn voetbalcarrière is door deze aanwijzing niet beter geworden. Tijdens mijn promotie heb ik er des te meer aan gehad. Ook de woorden "the work isn't finished until the paperwork is done" zijn meer dan waar gebleken. Ik wil u enorm bedanken voor de mogelijkheid die u mij heeft gegeven om mijn promotie in Maastricht voort te zetten.

Dr. Schroen, beste Blanche. Collega van het eerste uur. Eerst als promovendus, nu als mijn copromotor. Tijdens de AlO-etentjes had ik nooit gedacht dat we samen in een promotieteam zouden eindigen, laat staan in een Opel Zafira op weg naar Zwitserland. Het kan soms raar lopen. Met de terugkeer van je Londens intermezzo kreeg de groep een nieuwe moleculair biologische impuls. Jij introduceerde de microRNAs met succes in mijn langstlopende project. Hoofdstuk 5 is van ons! Belangrijker nog ben je geweest bij de laatste loodjes van dit proefschrift. Je hebt bewezen dat begeleiden op afstand kan. Ik kon altijd op je reactie rekenen bij een belletje of mailtje. Het waren vaak de extra zetjes om alles succesvol af te ronden. Bedankt voor al je steun.

Rick, jij bent het onbetwiste alfamannetje van het cardiolab. Verantwoordelijk voor de wetenschappelijke opvoeding van vele nieuwe promovendi en studenten. Ik heb altijd 
met bewondering gekeken hoe jij de balans tussen de nodige labdiscipline en humor weet te handhaven. Naast de zelfgekroonde koning van het 'western blotten' ben je de prins van de celkweek. Jouw filosofie over het omgaan met cellen heb ik geadopteerd en probeer ik nu door te geven aan volgende generaties. Helaas heb ik moeten constateren dat de methode van het 'naakt kweken' tot een ambacht is verworden en beperkt is gebleven tot Maastrichtse contreien. Je western blot capaciteiten zijn alom geroemd, al is tot op heden bij niemand duidelijk wat er precies gebeurt in de doka. De meest onmogelijke detecties lijken in jouw handen een succes te worden. Zonder jouw 'hand van de meester' had de revisie van hoofdstuk 5 er waarschijnlijk heel anders uit gezien. Rick, heel erg bedankt voor alles op het lab en daarbuiten!

Wouter, ik weet niet goed waar ik de afgelopen jaren meer steun aan heb gehad; jouw kundigheid op het lab en bereidheid om een helpende hand te bieden of je scherpzinnigheid en geweldige gevoel voor humor. Zeker is dat jij de revisies van het TSP-2-doxo en miRNA-aging artikel een stuk minder stressvol hebt gemaakt. Samen met jou op het lab staan was altijd een groot feest. Het isoleren van RNA was voor ons al lang geen uitdaging meer, top-40 liedjes voorzien van nieuwe teksten des te meer. Austin Powers kan ik al een hele tijd niet meer kijken zonder aan jouw 'Mi-cro-R-N-A' imitatie te denken. Naast mijn bewondering voor je wetenschappelijke activiteiten, ben je voor mij de 'wizard-of-Ableton'. Wellicht dat we als Wuftel en DJ Scheefmixer ooit nog een groter publiek bereiken dan met onze publicaties. Wouter, wetenschappelijk, muzikaal en qua humor kan ik altijd op je terugvallen. Ik ben er daarom trots op dat je 16 mei als mijn paranimf achter mij staat.

Nard, Nardmans, Professor, Mister 96, N.A.R.D., de vele bijnamen illustreren ons verleden samen. Al vanaf mijn stage bij cardiologie mijn wetenschappelijke mentor. De vastberadenheid waarmee jij een planning kan maken, er weer van af kan wijken, en nog drie keer kan bijstellen heeft mijn grote bewondering. Hoe je groots moet denken in de opzet van experimenten en het uitvoeren van celkweek op productieschaal is mij altijd bijgebleven. Ik heb veel van je geleerd, heel veel. Toen we in Würzburg samen zijn doorgezakt in de hotelbar, was ik dan ook blij dat ik jou ook nog iets kon leren. Helaas heb ik je nooit kunnen leren koffie drinken en was jouw hart verknocht aan de warme chocomel. Onze 'afternoon-gesprekken' hebben hier echter niet onder geleden. Nard, vanuit de Verenigde Staten van de US of A is je rol als wetenschappelijk mentor iets afgenomen. Gelukkig heb ik er wel een hele goede vriend aan over gehouden. Het is een eer dat je mijn paranimf wilt zijn.

Mark, Cellings, Bossche Bal, jij bent persoonlijk verantwoordelijk voor mijn introductie op het cardio-lab. Jij bent het bewijs dat er slechts een dunne scheidingslijn loopt tussen enthousiasme en fanatisme. Ongevraagd heb je mijn leven verrijkt met Brabantse trots, uitspraken als "extreem Italiaans", bijbelse citaten, rituitslagen van de Tour de France uit vervlogen tijden, vernieuwde voetbal inzichten en tal van andere feiten die 
doorgaans door niemand worden onthouden. Ik heb er van genoten! Het was een voorrecht om de trein terug naar het Brabantse met je te mogen delen en te filosoferen over wetenschap, voetbal, het lab, etc. Helaas hebben we het niveau van Descartes of Erasmus nooit gehaald. Als collega, maar vooral als goede vriend, was je altijd bereid om mee te denken met mijn projecten. Hoofdstuk 2 was ons eerste gezamenlijke wetenschappelijke wapenfeit. Daarnaast is jouw optimisme de basis geweest voor hoofdstuk 5. Hopelijk kunnen we er nog een succes aan toevoegen met een nieuw Veldhoven-Eindhoven initiatief. Mark, bedankt voor je positieve invloed op mijn promotie.

Maarten, mijn roommate gedurende de laatste loodjes. We hebben samen wat afgevochten over de ideale kamerindeling en het opbergsysteem voor al jouw ordners. Uiteindelijk was het mijn vers gezette koffie met op de achtergrond jouw Ella Fitzgerald, die bij tijden ons kantoor veranderden in een sfeervol bruincafé. Met de fles Corstendonck als één van onze grootste trofeeën. Wanneer jij dan ook nog je oranje trui en 'party-pants' aan had kon mijn dag helemaal niet meer stuk. Dat je naast dit alles ook nog een gedreven wetenschapper bent met een luisterend oor voor zijn collega's, maakt dat ik ontzettend heb genoten van onze tijd samen bij cardiologie. Maarten, heel erg bedankt voor alles en succes met het afronden van je promotie en de volgende stap. Ik hoop nog veel Corstendonkjes met je te mogen delen.

Barbara, de vraagbaak voor al uw promotievragen, met het vermogen om orde te scheppen in de wetenschappelijk organisatorische chaos. In het ziekenhuis liep ik graag een stukje verder om even gedag te zeggen. Geweldig hoe onze gesprekken vaak begonnen met een serieuze vraag en eindigden in complete onzin. Barbara, heel erg bedankt voor al je hulp bij het afronden van mijn promotie. Zonder jou had ik waarschijnlijk nog een aantal handtekeningen tegoed.

Anna, jouw komst gaf het lab pas echt internationale allure. Nederlandse, Belgische, Griekse en Engelse invloeden verenigd in een postdoc. Ondanks de geringe overlap van onze projecten was je altijd geïnteresseerd in mijn promotie en ben je verantwoordelijk geweest voor het transport van tal van 'stalen' tussen Maastricht en Leuven. Bedankt voor alles en ik zal de bbq in de heuvels van Cannes nooit vergeten.

Melissa, het is mij nog steeds niet duidelijk of onze promoties nu parallel verliepen of dwars door elkaar heen. Getuige hoofdstuk 3 hebben we zeker de nodige tijd samen doorgebracht. Zonder jouw hulp bij het TSP-2-doxo project had het in vivo gedeelte er heel anders uitgezien. Zowel in het lab in Leuven als tijdens ons congres in München ben je mijn gids geweest, met wanneer nodig een pleister voor op de wonden. Mijn dank is groot! 
Davy, ik bewonder jouw manier om dingen grondig uit te zoeken en vooral de wijze waarop. Jouw manier van werken mag voor een buitenstaander dan hectisch overkomen, maar aan het einde van de rit blijkt er dan toch een onderliggend systeem in te zitten. Hoofdstuk 6 is hiervan het bewijs. Davy, bedankt voor al je hulp en heel veel succes met je carrière.

Lucas, onze samenwerking ligt in het verlengde van hoofdstuk 6 . Waar onze samenwerking in het lab slechts zijdelings plaatsvond, waren we als voetbalteam tijdens mijn laatste lab-uitje onoverwinnelijk. Ik wens je veel succes met je promotie.

Paolo, the last but definitely not the least part of the Leuven-connection. You provided me with detailed day-to-day overviews of all ongoing animal experiments. I can't thank you enough for your contribution to the TSP-2-doxo studies. I wish you all the best.

Robert en Casper, de 'witte-jassen brigade'. Jullie hulp bij de selectie en verzameling van DCM-patiënten was onmisbaar. Heel veel succes met jullie carrière.

Marieke, Wouter en Mathijs, ik heb slechts kort met jullie mogen samenwerken. Onze ervaringen tijdens carnaval zijn des te onvergetelijker. Tot volgend jaar, achterin de Perroen.

Natuurlijk ben ik ook mijn collega's uit de 'oude doos' niet vergeten. Joost, als "zwaar sfeer verhogende vogel" was jij beslist de 'grutto' van het cardio-lab te noemen. Het deel dat ik me nog kan herinneren van de avond dat je hebt geprobeerd om mij te leren pokeren, zal ik nooit vergeten. Esther 'coffee' Creamers, mijn eerste ervaring met een 'Amerikaanse' postdoc. Jouw manier van onderzoek doen heeft een overweldigende indruk achtergelaten. We zien elkaar zeker volgend jaar met carnaval. Rudy, ruwe bolster blanke pit. Onmisbaar voor al mijn kloneringen en die ene keer in Nice dat ik de brandkraan niet dicht kreeg. Professor Pinto, beste Yigal, bedankt dat je mij de mogelijkheid hebt gegeven om mijn promotie te starten bij cardiologie. Gonda, mijn oude buurvrouw en gids in het dierenlab van farmacologie. Bedankt voor al je hulp met mijn muizenstudie en natuurlijk de histologie. Sylvia, mijn andere buurvrouw. Niemand kan zoveel RNA halen uit zo weinig weefsel! Jij bent van grote waarde geweest voor hoofdstuk 5 en 6 . Ik zal de vrijdagmiddag hakkuh-uurtjes nooit vergeten. Bedankt voor alles.

Ook mijn collega's van de afdeling pathologie Erwin, Jeffrey en Linda mogen niet ontbreken in dit dankwoord. Bedankt voor al jullie hulp bij mijn vragen over antilichamen en histologie protocollen. Jack, een bijzonder woord van dank voor al die keren dat de microscoop opnieuw moest worden ingesteld en voor jouw optreden als 'Leica Qwingoeroe' bij mijn histologie analyses. 
Mijn collega's van BMT. Wim, bedankt voor je assistentie bij mijn confocale microscopie sessies. Ik verbaas me er nog steeds over dat je het durfde om mij met de microscoop alleen te laten. Professor van Zandvoort, beste Marc, je was altijd geïnteresseerd en bereid om mee te denken met mijn project. Bedankt voor je enthousiasme.

Mijn collega's van de electronenmicroscopie. Hans, heel erg bedankt voor de vele foto's die je voor mijn studies hebt gemaakt. Dr. Verheyen, beste Fons, bedankt voor al je hulp bij het analyseren van de electronenmicroscopie foto's.

Verder wil ik graag Prof. Brunner-La Rocca, Prof. ten Cate, Prof. Schrauwen, Prof. Lijnen en dr. van Bilsen hartelijk danken voor het beoordelen van dit proefschrift.

Mijn collega's van de 'supramolecular medicine-groep' van de TU/e; Björne, Maartje, Bram, Lorenzo, Roxanne, Mellany, Isja en Jolanda. Jullie hebben mij als 'bioloog' geadopteerd in jullie 'scheikunde' midden. ledere werkbespreking leer ik weer bij van jullie. Bedankt voor al jullie interesse in mijn promotie. Professor Meijer, beste Bert, bedankt voor de kans die je mij hebt gegeven om binnen het SMO en het ICMS onderzoek te doen. Dr. Dankers, Patricia, vanaf ons eerste gesprek tijdens mijn sollicitatie voel ik mij thuis binnen de 'supramolecular medicine-groep'. Bedankt voor al het vertrouwen en de ruimte die je mij hebt gegeven om mijn promotie af te ronden.

Voor een succesvol promotietraject moet tegenover alle inspanning de nodige ontspanning staan. Ruud \& Hanneke, Deef (DJ Devotion) \& Kaatje, Erik (Pirke) \& Laura, Jeroen \& Ilse (de Vosjes) en Martijn (Stiffie), mijn promotie was voor jullie toch vooral 'iets met muizen', 'laboratorium' en het 'het hart of zoiets'. Desondanks bleven jullie altijd geïnteresseerd. Deef, bedankt dat je af en toe even een 'bakkie' kwam doen wanneer ik thuis mijn proefschrift aan het schrijven was. Pirke, goed om te weten dat wanneer ik mijn onderzoek niet meer kan uitleggen jij wel weet hoe het ook al weer zat... of zoiets. Stiffie, als lachen spierblessures zou voorkomen hadden we de afgelopen seizoenen meer gespeeld.

Mijn familie, een bijzonder 'cluppie' en ontzettend belangrijk voor mij. John, Elly, Marijke en Jeanne bedankt voor al jullie interesse. Gerrie en Inge, heel erg bedankt voor al jullie support; deze neef gaat eindelijk 'afstuderen'! Carla en Willem, zoveel meer dan mijn tante en oom, jullie steun en bijdrage gaat verder dan alleen de jaren van mijn promotie. Deze promotie is dan ook een beetje van jullie. Willem, het moment waarop je zei dat je het niet meer leuk vond om met mij te discussiëren, wist ik dat ik goed bezig was.

Mijn schoonouders, Frank en Henriëtte, jullie hebben alle 'ups' en 'downs' tijdens mijn promotie meegemaakt. Ondanks mijn eigenwijsheid zijn jullie altijd even betrokken gebleven. Indonesië was de 'break' die ik nodig had en een van de hoogtepunten van 
afgelopen jaren. Ik hoop er nog veel met jullie te mogen delen. Ilse \& Tim, Illy \& Timmie, of het nu een vakantie is, een avondje stappen, Extrema, carnaval, Koninginnedag, gewoon uit eten of op de bank hangen, het is altijd gezellig. Bedankt voor alle steun tijdens mijn promotie. Ik kijk uit naar onze reis naar Borneo en Sulawesi. Sandra, ondanks dat je aangaf nooit te snappen waar ik mee bezig was, was je enthousiasme en interesse er niet minder om. Ronnie \& Jamy \& Joe, de etentjes thuis zijn zoveel leuker met een volle eettafel. Alle uitjes naar PSV, 'gewoon even een biertje'momenten en op zijn tijd een vrijgezellenfeest, waren een welkome afleiding. Ik hoop nog veel van deze momenten met jullie te kunnen delen. Ik ben trots op onze 'big family'. Tim en Vera, last but not least. Tim, de laatste aanwinst van de familie, jouw humor is bij vlagen onnavolgbaar. Ik bewonder je positieve kijk op dingen en het vermogen om in elke situatie een kans te zien. lets waar ik nog veel van kan leren. Vera, al een aantal jaren geen Veertje meer. Ik moet je gelijk geven; het is allemaal goed gekomen. Met heel veel plezier en bewondering zie ik hoe je bezig bent met je nieuwe liefde, fotografie; met de omslag van mijn proefschrift als persoonlijk hoogtepunt. Vera, zusje, ik ben trots op alles wat je doet. Ik kan niet wachten op jullie gezinsuitbreiding. Stiekem hoop ik dat hij/zij net zo 'gek' wordt als zijn/haar vader en moeder.

Mijn ouders, Mam en Ruud, ik kan moeilijk onder woorden brengen hoeveel jullie voor mij betekenen. Jullie hebben mij altijd gesteund in iedere keuze die ik heb gemaakt. Ruud, ik heb grote bewondering voor de manier waarop jij de dingen 'managet'. Je bent daarmee voor een groot deel verantwoordelijk voor mijn 'persoontje en maniertjes'. lets waar ik heel erg trots op ben. Mam, je hebt het weer geflikt! "Moeders weten alles", dat is een veilig gevoel wanneer ik het even niet meer wist. Je bent de liefste moeder die ik me kan wensen. Mam en Ruud, ik ben er trots op dat jullie mijn ouders zijn.

De laatste woorden van dit proefschrift zijn voor mijn meisje. Het is onmogelijk om tijdens een promotie werk en privé gescheiden te houden. Je bent altijd de stille motor op de achtergrond geweest. 'Gas terug' wanneer het kon, 'gas erbij' wanneer ik dreigde stil te vallen. Niemand kent mij zo goed als jij; met alle grapjes, maar zeker ook met alle humeurtjes. Op de drukke momenten was jij mijn rustpunt, op de rustige momenten mijn maatje waar ik zo enorm mee kan lachen; "mensen zouden ons eens moeten zien met zijn tweeën". Deze promotie is ook van jou! Lieve Leanne, ik hou van je. Niet vergeten dat we pas net begonnen zijn. "Never change a winning team!"

Geert 


\section{List of publications}

Kieltyka RE, Bastings MM, van Almen GC, Besenius P, Kemps EW, Dankers PY. Modular synthesis of supramolecular ureidopyrimidinone-peptide conjugates using an oxime ligation strategy. Chem Comm (Camb). 2012 Feb;48(10):1452-1454

van Almen GC*, Swinnen $M^{*}$, Carai P, Verhesen W, Cleutjens JPM, D'hooge J, Verheyen FK, Pinto YM, Schroen B, Carmeliet P, Heymans S. Absence of thrombospondin-2 increases cardiomyocyte damage and matrix disruption in doxorubicin-induced cardiomyopathy. J Mol Cell Cardiol. 2011 May;51:318-328

van Almen GC, Verhesen W, van Leeuwen RE, van de Vrie $M$, Eurlings $C$, Schellings MW, Swinnen M, Cleutjens JP, van Zandvoort MA, Heymans S, Schroen B. MicroRNA18 and microRNA-19 regulate CTGF and TSP-1 expression in age-related heart-failure. Aging Cell. 2011 Oct;10(5):769-779

Kubben N, Voncken JW, Demmers J, Calis C, van Almen GC, Pinto YM, Misteli T. Identification of differential protein interactors of lamin A and progerin. Nucleus. 2010 Nov;1(6):513-525

Schellings MW*, Vanhoutte D*, van Almen GC, Swinnen M, Leenders JJ, Kubben N, van Leeuwen RE, Hofstra L, Heymans S, Pint YM. Syndecan-1 amplifies angiotensin IIinduced cardiac fibrosis. Hypertension. 2010 Feb;55(2):249-256

Swinnen $M^{*}$, Vanhoutte $D^{*}$, van Almen GC, Hamdani N, Schellings MW, D'hooge J, van der Velden J, Weaver MS, Sage EH, Bornstein P, Verheyen FK, VandenDriessche T, Chuah MK, Westermann D, Paulus WJ, van der Werf F, Schroen B, Carmeliet P, Pinto YM, Heymans S. Absence of thrombospondin-2 causes age-related dilated cardiomyopathy. Circulation. 2009 Oct;120(16):1585-1597

Schellings MW*, van Almen GC*, Sage EH, Heymans S. Thrombospondins in the heart: potential functions in cardiac remodeling. J Cell Commun Signal. 2009 Dec;3(3-4):201213

*Authors contributed equally 



\section{Curriculum Vitae}

Geert van Almen was born on April $13^{\text {th }}, 1983$ in Eindhoven, the Netherlands. In 1995, he attended the Stedelijk College Eindhoven in Eindhoven, where he graduated in 2001. His broad interest in the different aspects of health and disease brought him to Maastricht where he started his study Health Sciences at Maastricht University. During the last 2 years of his study he focused on the molecular biological aspects of health and performed an internship at the department of Experimental and Molecular Cardiology, where he was involved in the identification and characterization of lamin $A / C$ interacting partners and their role in laminopathies. After his graduation in Biological Health Sciences in 2005, he continued his research within the department of Experimental and Molecular Cardiology with a PhD position. His main focus was to unravel aging mechanisms in the heart with particular interest in the aging processes in the cardiomyocyte that determine why some people's heart ages at a faster rate than others. After two years he extended this research with a focus on the interplay between non-structural matrix proteins and the cardiac cells during cardiac remodeling. In 2009 he was awarded for best oral presentation during the CBCS/ESC Summer School on Cardiovascular Sciences. Since April 2010, he continues his research at the Laboratory of Chemical Biology and the Institute for Complex Molecular Systems at the Eindhoven University of Technology. His current research takes place in the area where chemistry meets biology and biomedical engineering and focuses on the design of novel biopolymers that can be applied as instructive scaffolds for tissue engineering. 